Multi-host viruses in Argentine ants and honey bees:

Increased viral disease in honey bees is associated with Argentine ants

by

Jana Dobelmann

\author{
A thesis \\ submitted to the Victoria University of Wellington \\ in fulfilment of the requirements for the degree of \\ Doctor of Philosophy \\ in Ecology and Biodiversity
}

Victoria University of Wellington

Te Herenga Waka 
This thesis was conducted under the supervision of

\author{
Professor Philip J. Lester (primary supervisor) \\ Victoria University of Wellington, \\ Wellington, New Zealand
}

and

Dr Antoine Felden (secondary supervisor)

Victoria University of Wellington,

Wellington, New Zealand 


\section{Abstract}

Emerging infectious diseases threaten public health, livestock economies, and wildlife. Human-mediated species introductions can alter host and pathogen communities that shape the dynamics of infectious diseases. Several RNA viruses that have been linked to population declines in wild pollinators and losses of managed honey bees have been detected in multiple other species and are suspected to circulate within insect communities. Yet, we lack an understanding of how disease dynamics are affected by the introduction of novel species. These introduced species include invasive ants, which can disturb honey bees and become a pest in apiaries. The Argentine ant (Linepithema humile) is a globally successful invader that has been observed to attack bees and multiple bee-associated viruses have been detected in this ant species.

Here, I studied interactions between Argentine ants and European honey bees (Apis mellifera) and how these interactions affect viral dynamics in beehives. I first tested a range of pollinators and associated insects for RNA viruses that are pathogenic to honey bees. Beeassociated viruses showed evidence for active viral replication in several pollinator species but also in species that cohabit in beehives such as ants, spiders, and cockroaches. Using phylogenetic analyses, I found that viral transmission within communities was shaped by geographic origin rather than being restricted by species barriers. Next, I used a longitudinal field study to test whether Argentine ant presence affected pathogen infections and survival in beehives. Argentine ants tested positive for three bee-associated viruses even before beehives were moved into ant-infested sites. Increased levels of deformed wing virus in beehives in autumn were associated with ant presence, although hive mortality was not affected by ants over the duration of this experiment. I used RNA sequencing on a subset of honey bee samples collected during autumn to study the RNA virome and identify transcriptomic responses associated with ant presence. Twelve RNA viruses were found in beehives, among those, three plant-associated viruses and an unclassified RNA virus that had not previously been observed in honey bees. Deformed wing virus showed the highest viral titres in most hives, but was only 
marginally affected by ant presence. Sacbrood virus and tomato ringspot virus levels were increased in hives with ants, however, both viruses are not known to infect Argentine ants and the plant-associated tomato ringspot virus seems unlikely to affect bee health.

Lastly, I tested the feasibility of controlling Argentine ants in apiaries using a novel pest control strategy. RNA interference is a conserved cellular gene regulation mechanism that could be used to silence specific genes in ants. Using double-stranded RNA (dsRNA) to silence two immune-related genes in Argentine ants was expected to increase pathogen susceptibility, which could then lead to higher pathogen levels that reduce ant numbers. My results indicated that no consistent immune silencing could be achieved in the field. Immune gene expression changes were observed, but pathogen titres were not affected, and ant numbers stayed high. Argentine ant control using a conventional insecticide significantly increased bee survival, whereas many hives in the dsRNA and control group abandoned their hives due to ant attacks. Although population control was not successful using the two Argentine ant-specific dsRNAs, insights into ant immunity and ant-bee interactions could improve the development of novel control strategies.

Bee-associated viruses have repeatedly been detected in ant species, yet, this is one of the first studies to investigate whether ants affect viral dynamics in honey bees. I showed that invasive Argentine ants are associated with increases in viral pathogens in honey bees. The mechanisms by which ants affect bee disease are unknown, although there is some evidence for ants transmitting viruses or causing stress responses in bees that affect immunity. The findings of this thesis highlight the risk of invasive ant species disrupting pollination services. New and environmentally-friendly methods to control invasive species are urgently needed to improve bee health and limit the spread of invasive ants, such as Argentine ants. The high prevalence of bee-associated viruses and viral diversity in ants suggests that pathogens that are suitable for population control might be present in ant populations, although risks of spillovers into other species need to be carefully considered. 


\section{Acknowledgements}

First, I want to thank my supervisor Professor Dr Phil Lester for the opportunity to do this research. I feel very lucky to have had Phil as my supervisor, his help and guidance has always been fantastic and provided the perfect balance between support and freedom. I would also like to thank my secondary supervisor, Dr Antoine Felden, for his encouragement and guidance, for his expertise with R, his help with RNA-seq and for joining me on countless field trips to the Far North.

Thanks to everyone who helped in the field and tolerated bee stings and the hot Northland weather: Brittany Florence-Bennett, Jess Russell, Evan Brenton-Rule, and of course Antoine!

Thanks to Bruce and Craig Robertson for providing beehives, for allowing me to access their apiary sites and for being so welcoming. I really appreciate your help!

I am thankful to everyone in the lab group for their helpful feedback on drafts, for stimulating discussions during group meetings and for lots of yummy baking: Mariana Bulgarella, James Baty, John Haywood, Rose McGruddy, Matt Howes, Zoe Smeele and Julia Eloff. Thank you, James, for your help with the taqman array. Special thanks to Rose for proofreading parts of this thesis and for being the best (at Catan).

I am grateful for the financial support provided by the Victoria Doctoral Scholarship. This research was funded by a Royal Society of New Zealand Marsden Grant and a Centre for Biodiversity and Restoration Ecology student award partly funded the RNA sequencing.

Thanks to Mary Murray, Mark Stephen and the SBS admin team for keeping things running!

I'd like to thank my friends and colleagues at SBS for morning coffee and all the other distractions that make the workday much more enjoyable. Thanks to the quarantine crew for being so supportive when we were suddenly all stuck at home. Thank you, Sonali, for being my gym buddy and always being full of energy!

Thanks to my friends who were always there for me throughout this journey and joined me on many trips to go tramping, snorkelling or to just spend the weekend away at a bach. Thank you, Irina, Andy, Abi, Colan, and Lucy! Thanks also to my friends and family back home, whom I can always rely on. Special thanks to Fabienne Maihoff for visiting me in NZ!

Finally, I'd like to thank my parents, Marion and Detlef, and my sister Svenja for their ongoing support and for always believing in me. Danke, dass ihr immer für mich da seid! 


\section{Table of contents}

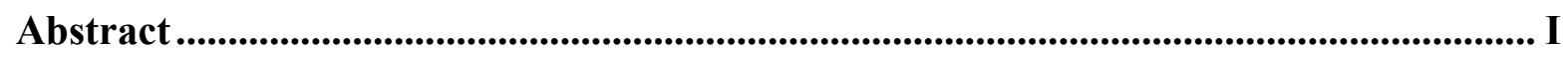

Acknowledgements............................................................................................................... III

Table of contents............................................................................................................

List of figures ..................................................................................................................................

List of tables .....................................................................................................................................XI

List of supplemental materials ............................................................................................. XII

List of virus abbreviations .....................................................................................XIV

List of other abbreviations ............................................................................................. XV

Statement of contributions to this thesis .............................................................................XVI

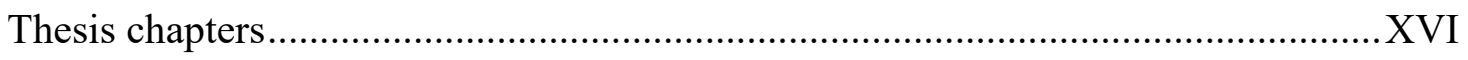

Publications included in this thesis .......................................................................

Chapter 1: General introduction............................................................................................ 1

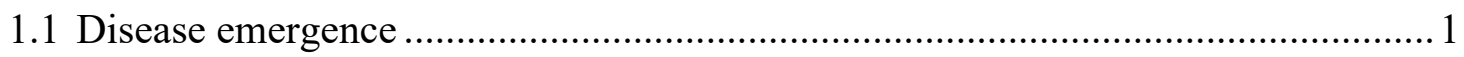

1.2 Declines in pollinator abundance and honey bee health are linked to emerging RNA

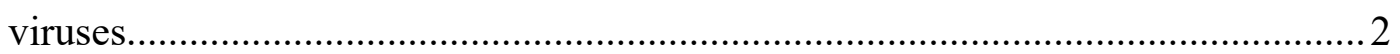

1.3 Lessons from Varroa and the re-emergence of deformed wing virus in honey bees 4

1.4 Species invasions can alter disease dynamics ................................................ 5

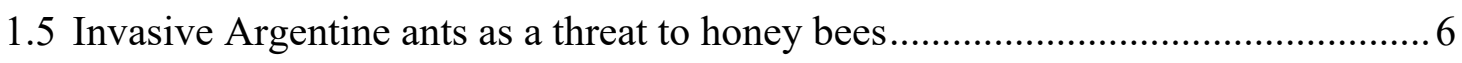

1.6 Harnessing viral pathogens to improve Argentine ant control in apiaries ............... 7

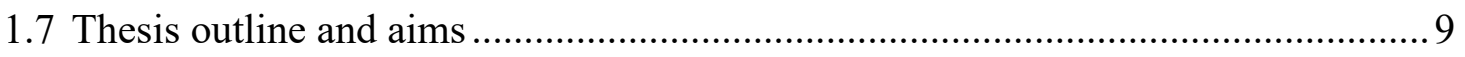

Chapter 2: Genetic strain diversity of multi-host RNA viruses that infect a wide range of pollinators and associates is shaped by geographic origins...........................11

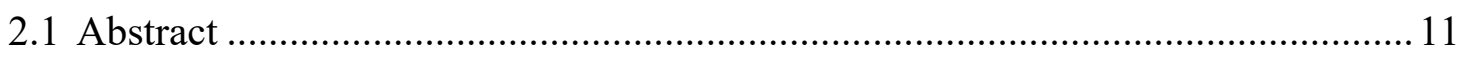

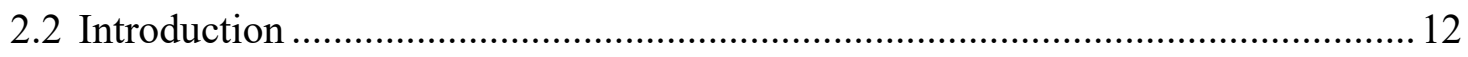


2.3 Materials and methods 14

2.3.1 Specimen collections 14

2.3.2 RNA extraction and reverse transcription PCR for virus detection. 15

2.3.3 Negative strand detection. 16

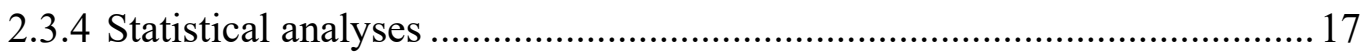

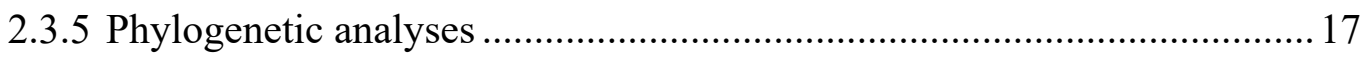

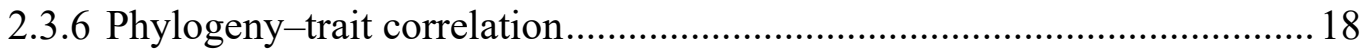

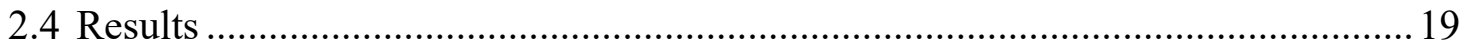

2.4.1 Viral prevalence in pollinators and associated arthropods ...................... 19

2.4.2 Viral coinfections in honey bees and Argentine ants............................... 19

2.4.3 Viral replication within host species...................................................20

2.4.4 Viral strain diversity and phylogenetic analyses .................................. 21

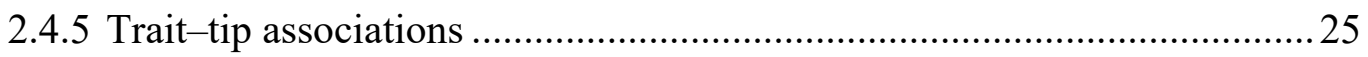

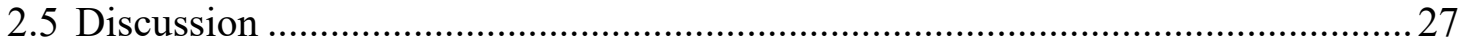

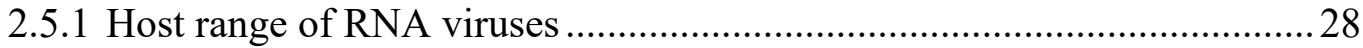

2.5.3 Pathogen reservoir and viral spillover into wild populations ...................22

2.5.4 Coinfections and interactions among viruses .......................................2 29

2.5.5 Multi-host viruses in emerging disease ................................................. 30

2.5.6 Global distribution and evolution of bee viruses ..................................... 31

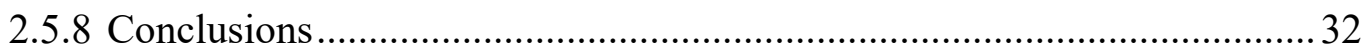

Chapter 3: Invasive ants are associated with elevated deformed wing virus infections in honey bees ..............................................................................................................................33

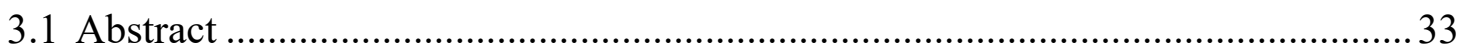

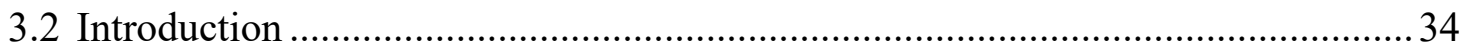

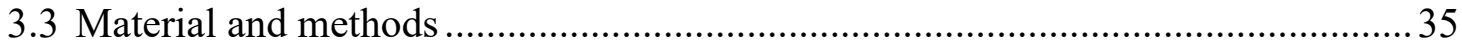

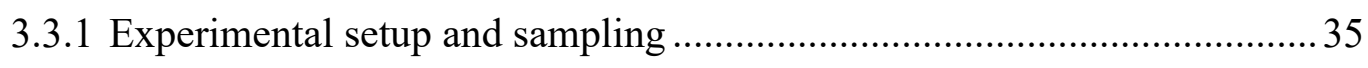

3.3.2 Homogenisation and RNA extraction ................................................. 37

3.3.3 Pathogen detection and quantification in honey bees ............................. 38 
3.3.4 Pathogen detection and quantification in Argentine ants

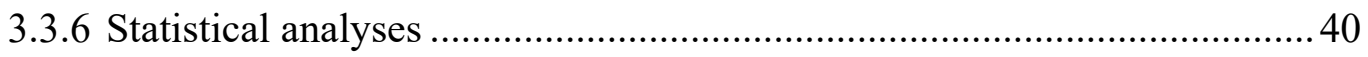

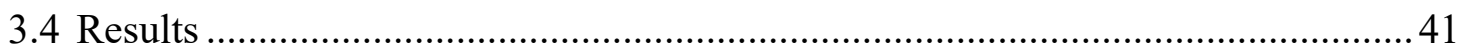

3.4.1 Hive survival and foraging activity................................................. 41

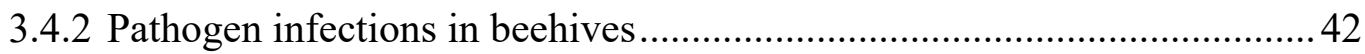

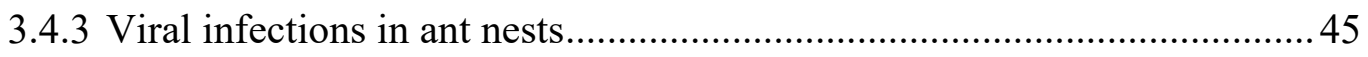

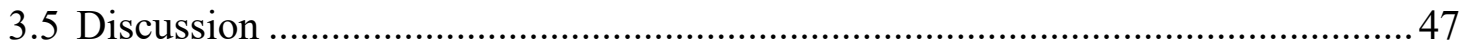

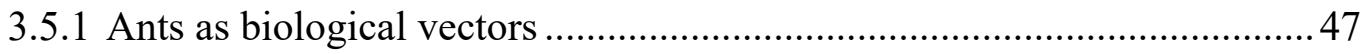

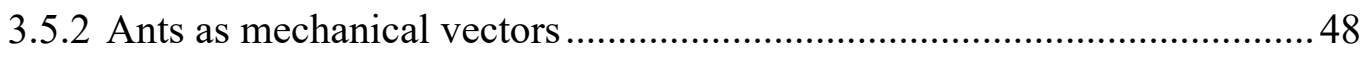

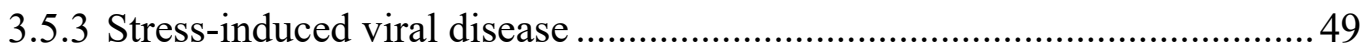

\section{Chapter 4:RNA sequencing reveals invasive ants are linked to increased Sacbrood virus} levels and trigger transcriptomic responses in honey bees.............................52

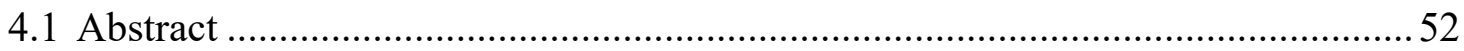

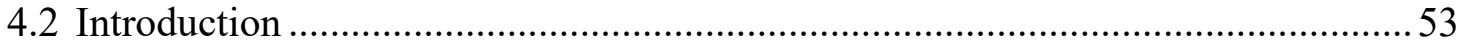

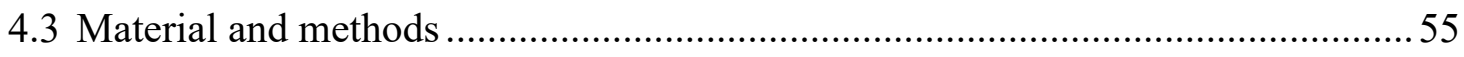

4.3.1 Experimental design and RNA library preparation .................................55

4.3.2 Quality control and ribosomal RNA filtering .......................................56

4.3.3 Honey bee transcriptome and differential gene expression ......................56

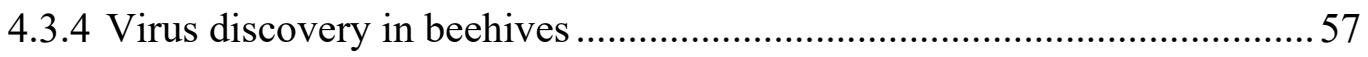

4.3.5 Viral infections in beehives with and without Argentine ants ..................57

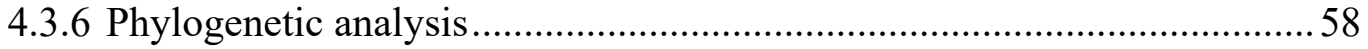

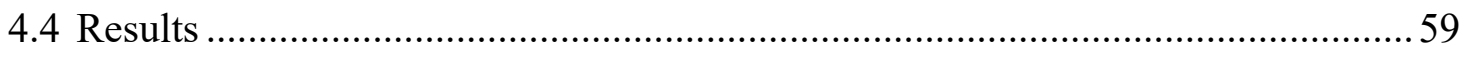

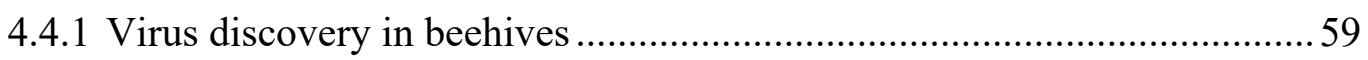

4.4.2 Effect of Argentine ants on viral pathogens in honey bees ..................... 61

4.4.3 Phylogeny of ALPV and an unclassified Riboviria ................................ 61

4.4.4 Differential gene expression in beehives with and without Argentine ants63

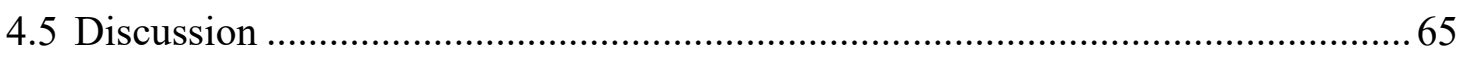

4.5.1 Virus discovery and the association between ants and viral titres............65 
4.5.2 Bees could potentially vector plant viruses ............................................ 66

4.5.3 Transcriptomic changes associated with Argentine ant presence..............67

4.5.4 Implications for understanding ant-bee interactions................................ 68

\section{Chapter 5:RNA interference, used to inhibit immune function, was not effective in} controlling Argentine ants in apiaries ...........................................................69

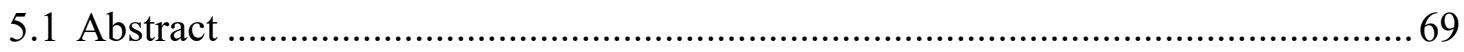

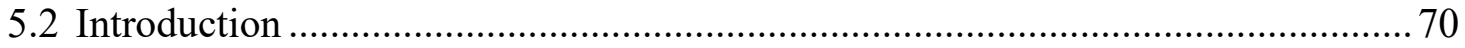

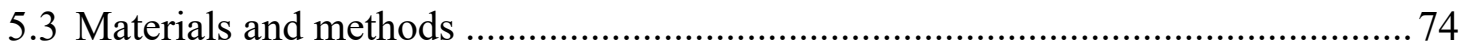

5.3.1 Double-stranded RNA bait and experimental design ............................. 74

5.3.2 RNA extraction and reverse transcription quantitative PCR ................... 77

5.3.3 Effects of dsRNA on gene expression and pathogen levels .................... 79

5.3.4 Effects of dsRNA and vanquish bait on ant numbers and hive survival ... 82

5.4 Results 83

5.4.1 Gene expression in Argentine ants treated with dsRNA 83

5.4.2 Viral and bacterial pathogen loads in Argentine ants treated with dsRNA86

5.4.3 Effects of dsRNA and vanquish bait on ant numbers 87

5.4.4 Effects of ant numbers, dsRNA and vanquish treatment on hive survival 88

5.5 Discussion 90

Chapter 6: General discussion ...................................................................................................94

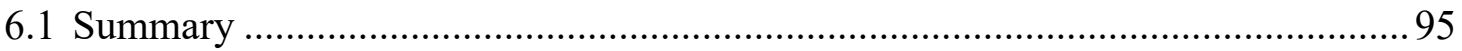

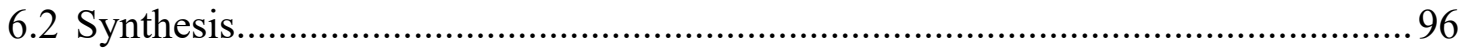

6.2.1 Do Argentine ants vector bee diseases? ...................................................96

6.2.2 Stressors can trigger viral diseases in honey bees ................................ 98

6.2.3 Do increased viral loads in association with Argentine ants affect hive mortality? 99

6.2.4 Argentine ant control relies on pathogenic viruses within ant populations100 6.3 Conclusion and outlook. 101 
References.

Supplemental materials ...................................................................................................................... 131

Supplemental figures and tables Chapter 2: ...................................................... 131

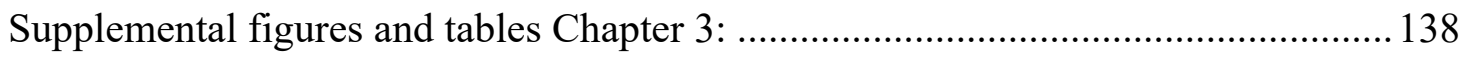

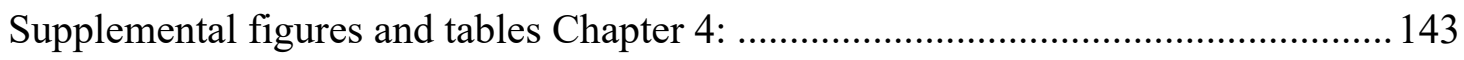

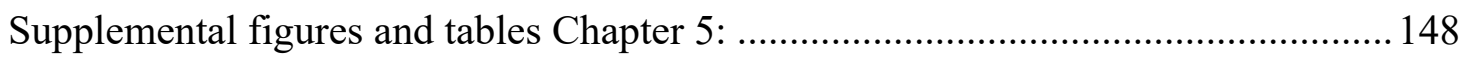

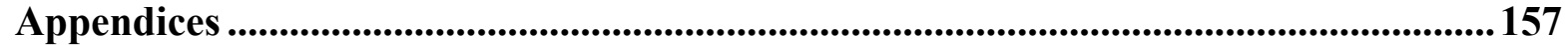




\section{List of figures}

Figure 2.1: Venn diagram of viral coinfection in honey bees (A. mellifera) (A) and Argentine ants (L. humile) (B).

Figure 2.2: Maximum clade credibility tree for the $R N A$-dependent $R N A$ polymerase (RdRp) fragment (440 bp) of deformed wing virus (DWV).

Figure 2.3: Maximum clade credibility tree for the major capsid protein $v p 3$ fragment (360 bp) of Kashmir bee virus (KBV).

Figure 3.1: Experimental design to test the effect of Argentine ants on viral infections in beehives.

Figure 3.2: Survival and foraging activity of beehives with and without Argentine ants..... 42

Figure 3.3: Pathogen load and prevalence in honey bee (A. mellifera) hives after beehives were placed in sites with and without Argentine ants (L. humile)......

Figure 3.4: Viral load and prevalence of bee-associated viruses in Argentine ants (L. humile) after beehives were placed next to ant nests. 46

Figure 4.1: Photos of beehives with (and without) Argentine ants (L. humile). 55

Figure 4.2: A heat map showing read numbers of viruses found in RNA sequencing libraries from beehives with and without Argentine ants.

Figure 4.3: Virus titres in honey bees (A. mellifera) from sites with and without Argentine ants (L. humile). 60

Figure 4.4: Maximum clade credibility tree for 1,931 nucleotides of the putative structural protein of Aphid lethal paralysis virus (ALPV) and the unclassified Hubei picorna-like virus 15 (HPLV15).

Figure 4.5: A heat map of the top 50 differentially expressed genes (DEGs) in honey bees with and without Argentine ants. 
Figure 5.1: Schematic overview of the hypothesised method to use RNA interference (RNAi) induced immunosuppression for Argentine ant pest control. .73

Figure 5.2: Experimental design to test double stranded RNA (dsRNA) for ant population control. 76

Figure 5.3: Experimental setup and Argentine ant (L. humile) sampling. 77

Figure 5.4: Differential gene expression of dicer-1 (A) and spätzle (B) in Argentine ants treated with a mix of double-stranded RNA (dsRNA) targeting dicer-1 and spätzle...... 83

Figure 5.5: A heat map showing gene expression and pathogen load in Argentine ants treated with a mix of double-stranded RNA dicer-1 and spätzle relative to a control group. . 85

Figure 5.6: Argentine ant abundance on bait dishes and beehives during the experiment. ..... 87

Figure 5.7: Observations of beehives with Argentine ants. 88

Figure 5.8: Kaplan-Meyer survival curves of 15 beehives after being moved into a field site with Argentine ants and controlling ants using three different treatments. 89

Figure 6.1: Argentine ants cause stress in honey bees through attacks on beehives that may be linked to changes in pathogen infections. 99 


\section{List of tables}

Table 2.1: Estimated viral prevalence in honey bees (A. mellifera) and Argentine ants $(L$. humile) for five virus targets with $95 \%$ confidence intervals (CI) in brackets. 20

Table 2.2: Viral presence in pollinators and associated arthropods tested for deformed wing virus (DWV), Kashmir bee virus (KBV), Moku virus (MKV), black queen cell virus (BQCV), and Linepithema humile bunya-like virus 1 (LhuBLV1) using RT-PCR.... 22

Table 2.3: Trait-tip association in deformed wing virus (DWV), as calculated with 500 random trees (replicates) in BaTS (Bayesian tip-association significance testing) (Parker et al. 2008).

Table 2.4: Trait-tip associations for Kashmir bee virus (KBV), as calculated with 500 random trees (replicates) in BaTS (Bayesian tip-association significance testing) (Parker et al. 2008).

Table 5.1: Grouping of genes and pathogens for statistical analyses

Table 5.2: Repeated measures permutational multivariate analysis of variance (PERMANOVA) testing the effect of double-stranded RNA, experimental phase and their interaction on gene expression and pathogen loads. .86 


\section{List of supplemental materials}

\section{Chapter 2:}

Figure S2.1: Sampling pollinators and associated arthropods in Northland in apiary- and nonapiary sites.

Figure S2.2: Sampling sites in the far north region near Ahipara, Northland, New Zealand.132

Figure S2.3: Non-dated maximum clade credibility tree for the $R d R p$ fragment (440 bp) of deformed wing virus (DWV) without tip dates.

Figure S2.4: Non-dated maximum clade credibility tree for the $v p 3$ fragment (360 bp) of Kashmir bee virus (KBV) without tip dates

Table S2.1: Primers used for virus detection.

Table S2.2: Sampling location of additional Argentine ant samples used for phylogenetic analyses from Felden et al. (2019).

Table S2.3: Trait-tip association of non-dated deformed wing virus (DWV) phylogeny, as calculated with 100 random trees (replicates) in BaTS (Parker et al. 2008). 136

Table S2.4: Trait-tip association of non-dated Kashmir bee virus (KBV) phylogeny, as calculated with 100 random trees (replicates) in BaTS (Parker et al. 2008). 137

\section{Chapter 3:}

Figure S3.1: Beehive sampling locations for longitudinal study in Northland in New Zealand.

Figure S3.2: Bee-infecting viruses in Argentine ant (L. humile) nests in apiaries and outside apiaries in March

Figure S3.3: Viral load and prevalence of ant-associated viruses in Argentine ant (L. humile) nests after beehives were placed next to the nests. 
Table S3.1: Primer sequences used in this study.

\section{Chapter 4:}

Figure S4.1: Beehive sampling locations in Northland in New Zealand. 143

Figure S4.2: Principal component analysis (PCA) of RNA sequencing reads that mapped onto the honey bee genome (left) and reads that did not map concordantly (right). 144

Table S4.1: Sequence library and alignment stats.

Table S4.2: Results from BLASTx searches of de novo assembled transcripts against the NCBI virus database (viral.nonredundant_protein.1.protein.faa).

Table S4.3: List of 141 differentially expressed genes between bees with and bees without Argentine ants with adjusted $\mathrm{p}<0.05$ and an absolute fold change $(\mathrm{FC})>2$. 146

\section{Chapter 5:}

Figure S5.1: Nonmetric multidimensional scaling ordination of ant nest gene expression and pathogen load based on Bray-Curtis dissimilarities on $2^{\Delta \mathrm{Cq}}$ values 150

Figure S5.2: The number of Argentine ants (A) and honey bees (B) on the outside of beehives that absconded or survived the course of the experiment.

Figure S5.3: Number of Argentine ants and honey bees on beehives that absconded (abandoned their hive) estimated from photo counts. 151

Table S5.1: Double-stranded RNA (dsRNA) targets used for Argentine ant control. 152

Table S5.2: TaqMan array targets used to measure gene expression and pathogen levels in Argentine ants. 153 


\section{List of virus abbreviations}

$\begin{array}{ll}\text { ABPV } & \text { acute bee paralysis virus } \\ \text { ALPV } & \text { aphid lethal paralysis virus } \\ \text { ArMV } & \text { Arabis mosaic virus } \\ \text { BQCV } & \text { black queen cell virus } \\ \text { CrPV } & \text { cricket paralysis virus } \\ \text { DWV } & \text { deformed wing virus } \\ \text { HPLV-15 } & \text { Hubei picorna-like virus 15 } \\ \text { IAPV } & \text { Israeli acute paralysis virus } \\ \text { KBV } & \text { Kashmir bee virus } \\ \text { LhuBLV1 } & \text { Linepithema humile bunya-like virus 1 } \\ \text { LhuCV1 } & \text { Linepithema humile C virus 1 } \\ \text { LhuEV1 } & \text { Linepithema humile entomopoxvirus 1 } \\ \text { LHUV1 } & \text { Linepithema humile virus 1 } \\ \text { LhuPcV1 } & \text { Linepithema humile polycipivirus 1 } \\ \text { LhuPcV2 } & \text { Linepithema humile polycipivirus 2 } \\ \text { LhuPiLV1 } & \text { Linepithema humile picorna-like virus 1 } \\ \text { LhuPLV1 } & \text { Linepithema humile partiti-like virus 1 } \\ \text { LhuRLV1 } & \text { Linepithema humile rhabdo-like virus 1 } \\ \text { LhuQLV1 } & \text { Linepithema humile qinvirus-like virus 1 } \\ \text { LhuTLV1 } & \text { Linepithema humile toti-like virus 1 } \\ \text { LSV } & \text { Lake Sinai virus } \\ \text { MKV } & \text { Moku virus } \\ \text { SBV } & \text { sacbrood virus } \\ \text { ToRSV } & \text { tomato ringspot virus } \\ \text { WCCV2 } & \text { white clover cryptic virus }\end{array}$


List of other abbreviations

AI

$\mathrm{AICc}$

BaTS

bp

cDNA

CI

$\mathrm{Cq}$

DEG

dsRNA

FC

HPD

Imd

JNK

$\mathrm{MC}$

mRNA

miRNA

PAMP

PBS

PCA

PERMANOVA

PP

PPI

PS

$\mathrm{RdRp}$

RNAi

RNA-seq

rRNA

RT-PCR

SE

siRNA

$(+)$ ssRNA

VSRs

qPCR association index

corrected Akaike information criterion

Bayesian tip-association significance testing

base pair

complementary DNA

confidence interval

cycle quantification

differentially expressed gene

double-stranded RNA

fold change

highest posterior density

immune deficiency

c-Jun N-terminal kinases

monophyletic clade

messenger RNA

micro RNA

pathogen-associated molecular pattern

phosphate-buffered saline

principal component analysis

permutational multivariate analysis of variance

posterior probability

peptidyl-prolyl cis-trans isomerase like

parsimony score

RNA-dependent RNA polymerase

RNA interference

RNA sequencing

ribosomal RNA

reverse transcription polymerase chain reaction

standard error

small interfering RNA

(positive-sense) singe-stranded RNA

viral suppressors of RNA silencing

quantitative polymerase chain reaction 


\section{Statement of contributions to this thesis}

I hereby declare that this thesis is my own work and that all sources quoted, paraphrased or otherwise referred to haven been acknowledged in the references. To the best of my knowledge, this thesis neither contains material previously published or written by another person, nor material which has been accepted for the award of any other degree or diploma of the university or other institutes of higher learning.

\section{Thesis chapters}

Chapters 2, 3, 4, and 5 have been written as individual research papers. As all these chapters contain contributions from collaborators, they are written in first person plural tense.

Chapter 1: The general introduction was written in its entirety by Jana Dobelmann (JD).

Chapter 2: JD designed and carried out all the experimental and molecular work. Antoine Felden (AF) and Jess Russell assisted with sample collections in the field. Phil Lester (PL) and AF provided advice on the design of the experiment and the content of the manuscript. PL and AF contributed to the writing through editing drafts before submission to Viruses.

Chapter 3: JD designed and carried out all the experimental and molecular work with training and advice from PL and AF. AF assisted with the experimental setup and sampling of ants. PL and Evan Brenton-Rule occasionally assisted in sample collections. James Baty (JB) assisted with aspects of the molecular analyses by designing taqman assays for virus detection in ants. John Haywood $(\mathrm{JH})$ provided advice on statistical analyses. JD carried out all sample processing, molecular work, data collection and analysis, and wrote the chapter. PJ and AF advised on and edited earlier versions of the chapter.

Chapter 4: JD designed and carried out all the molecular and bioinformatics work with advice from PL and AF. JD successfully applied for extra grants from the Centre for Biodiversity and Restoration Ecology that helped cover the sequencing costs. BGI Genomics carried out sample library preparation. AF provided training and support for bioinformatics and 
sequencing analyses. JD carried out molecular work, data analyses, and wrote the chapter. PJ and AF advised on and edited earlier versions of the chapter.

Chapter 5: JD designed the experiment with PL and AF. AF designed dsRNA targets and conducted preliminary tests using these targets in the lab. JD carried out experimental work and AF and Brittany Florence-Bennett assisted with the experimental setup and sample collections. JD extracted RNA and JB designed taqman assays and carried out qPCR. JH provided advice with statistical analyses. JD analysed the data and wrote the chapter with guidance and input from PL and AF.

Chapter 6: The general discussion was written in its entirety by JD.

\section{Publications included in this thesis}

Appendix 1: Genetic Strain Diversity of Multi-Host RNA Viruses that Infect a Wide Range of Pollinators and Associates is Shaped by Geographic Origins by Jana Dobelmann, Antoine Felden, and Philip J. Lester as published in Viruses (12) pp 358, openly available under a Creative Commons Attribution License (CC BY 4.0). This research article was written by JD with contributions from PL and AF who editing drafts and advised on contents. Contributions to this article are listed under Chapter 2.

Appendix 2: Viruses and their effects in ants (Hymenoptera: Formicidae) by James W. Baty, Mariana Bulgarella, Jana Dobelmann, Antoine Felden, and Philip J. Lester as published in Myrmecological news (30) pp 213-228, openly available under a Creative Commons Attribution License (CC BY 4.0). This review article was jointly written by the co-authors. JD wrote the second and third paragraph in the "Methodology for viral discovery and delimitation in ant communities" section, contributed to figures and edited drafts before submission to Myrmecological news. 


\section{Chapter 1:General introduction}

\subsection{Disease emergence}

New and re-emerging infectious diseases threaten public health, livestock economies, and wildlife (Cleaveland et al. 2001, Dobson and Foufopoulos 2001). An emerging pathogen is defined as a pathogen with increasing incidence rates following its appearance in a new host population (Woolhouse and Dye 2001, Woolhouse 2002). Pathogen re-emergence occurs when incidence rates increase in an already existing population (Woolhouse and Dye 2001, Woolhouse 2002). Global travel and movement of animals and goods can quickly spread a disease that emerged anywhere on the planet over a large scale, requiring global surveillance and control networks (Fidler 1996). RNA viruses are particularly likely to emerge as infectious diseases as some of the most recent public health threats show (Cleaveland et al. 2001, Dobson and Foufopoulos 2001, Woolhouse 2002, Woolhouse et al. 2005). The emergence of the Severe acute respiratory syndrome coronavirus 2 (SARS-CoV2) and the global pandemic it is currently causing demonstrates how quickly a new viral disease can affect the lives of billions of people and how mitigating viral spread is extremely challenging (Rothan and Byrareddy 2020). Other public health threats are not new to humans but equally severe. The emergence and reemergence of the Ebola virus from animal reservoirs has previously caused several outbreaks since its first discovery in 1976, with the latest large outbreak spreading beyond West Africa in 2014 (Sanchez et al. 1995, Leroy et al. 2005, To et al. 2015). Virus reservoirs can provide a source from which viruses constantly spill into the host community and may re-infect members that have eliminated infections. Spillover events and host shifts are important in disease emergence and disease maintenance in populations (Woolhouse et al. 2005).

Wildlife populations can act as reservoirs for pathogens that infect domestic animals and humans (Daszak et al. 2000). One example is the rabies virus, with reservoirs in bats and wild carnivores from which the virus can be transmitted to domesticated dogs or directly to humans causing the often deadly Rabies disease (Calisher et al. 2006, Lembo et al. 2008). Another example is Mycobacterium bovis, the causative agent of bovine tuberculosis, which is 
suspected to have multi-host reservoirs in many countries (Palmer 2013). Confirmed host species include the European badger, African buffalo, wild boar, white-tailed deer and brushtail possum (Palmer 2013). The control of brushtail possums, the main wildlife vector of bovine tuberculosis in New Zealand, is part of the TBfree programme which aims to eradicate the disease from New Zealand by 2055 (Warburton and Livingstone 2015). Early detection of emerging pathogens through the identification of reservoir hosts and controlling contact rates between the reservoir and the recipient host can prevent disease outbreaks (McCallum and Dobson 1995, Parrish et al. 2008). The spillover from domesticated animals into wildlife populations can also threaten wildlife populations (Daszak et al. 2000, Fürst et al. 2014, Alger et al. 2019). Disease emergence in wildlife populations may not be as apparent at first but can be devastating to conservation and global biodiversity (Dobson and Foufopoulos 2001, Daszak et al. 2003). Multiple lines of evidence suggest that emerging diseases could play a key role in wild pollinator declines and the increased losses of managed pollinators that have been observed in recent years (Biesmeijer et al. 2006, Cox-Foster et al. 2007, Potts et al. 2010, Goulson et al. 2015).

\subsection{Declines in pollinator abundance and honey bee health are linked to emerging RNA viruses}

Several insect and pollinator communities have experienced recent population declines, leading to concerns about malnutrition, food security, and economic losses (Biesmeijer et al. 2006, Potts et al. 2010, Hallmann et al. 2017, Sánchez-Bayo and Wyckhuys 2019). Pollinators are indispensable for modern agriculture: $35 \%$ of the global food production and 87 out of 115 leading food crops worldwide depend on pollinators (Klein et al. 2007), accounting for an estimated global economic value of $€ 152.9$ billion each year (Gallai et al. 2009). European honey bees (Apis mellifera) are the most commonly managed pollinator species and are used to enhance yields in many food crops (Klein et al. 2007). Although wild pollinators and non-bee insects also significantly contribute to crop pollination, managed pollinators are increasingly used to supplement pollination and moved over large distances (Garibaldi et al. 2013, Rader et al. 2016). Managed honey bees in many countries have experienced substantial health declines 
(Evans and Schwarz 2011). According to a survey by the non-profit research association COLOSS (www.coloss.org), losses of overwintering hives around 15\% are common in many countries (Brodschneider et al. 2018, Gray et al. 2020). Particularly, high overwinter losses of more than $30 \%$ in Germany and more than $25 \%$ in Mexico, Spain and the USA have been observed in some years, while winter losses in other countries such as Norway and other Northern European countries remain below 10\% (Kulhanek et al. 2017, Brodschneider et al. 2018, Gray et al. 2020).

Increasing evidence points towards RNA viruses as a key driver of honey bee losses and wild pollinator declines (Manley et al. 2015, McMenamin and Flenniken 2018, Grozinger and Flenniken 2019). Many of the viruses found in honey bees belong to the Dicistroviridae and Iflaviridae families of positive-sense single-stranded RNA (+ssRNA) viruses (Evans and Schwarz 2011). These viruses are fast-evolving with high mutation rates caused by error-prone RNA-dependent RNA polymerases (Drake and Holland 1999). Many of these viruses have been detected in multiple pollinator species and are suspected to circulate widely within pollinator communities (Singh et al. 2010, Levitt et al. 2013).

The earliest discovery of viruses in honey bees goes back to the 1960s (Bailey 1967, Bailey 1968). Although bee-infecting viruses have been studied for decades, we are only beginning to understand why some viruses can be so deadly to bees and how they can be managed to improve bee health. Insect hosts are commonly coinfected with multiple viruses (McMahon et al. 2015). Most infections occur covertly without any obvious disease symptoms and have restricted viral replication (Chen and Siede 2007, Sorrell et al. 2009, Williams et al. 2017). Evidence suggests that stressors such as certain viruses, pesticides or poor nutrition have the potential to trigger a rapid increase in viral infection which can cause health declines and the sudden collapse or death of a bee colony (Cox-Foster et al. 2007). Increased viral titres in honey bees can facilitate horizontal disease transmission (Sorrell et al. 2009) and spillover into wild pollinator communities (Fürst et al. 2014, McMahon et al. 2015, Manley et al. 2019). What triggers mild infections into becoming severe outbreaks is poorly understood. Pathogens and 
other stressors interact and it is hypothesised that their effects synergise and are amplified by the interaction (Nazzi et al. 2012, Dolezal et al. 2019).

\subsection{Lessons from Varroa and the re-emergence of deformed wing virus in honey bees}

One of the best-studied examples of how a relatively benign honey bee virus became a global emerging disease that caused unprecedented colony losses is the global invasion of the Varroa mite (Wilfert et al. 2016). Varroa jumped species barriers from its original host, the Asian honey bee $A$. ceranae, to the European honey bee A. mellifera when European honey bees were introduced into Asia (Oldroyd 1999). European honey bees typically lack the hygienic behaviour required to efficiently detect and remove mites from nestmates, likely because the species did not co-evolve with the mite (Boecking and Spivak 1999). Consequently, Varroa populations increased rapidly and with the ongoing transport of honey bees around the world; Varroa mites now infest honey bee populations on almost every continent (except Australia) (Oldroyd 1999, Wilfert et al. 2016). The global introduction of Varroa had severe impacts on viral dynamics in bees through mites vectoring RNA viruses and actively suppressing immune function (Bowen-Walker et al. 1999, Yang and Cox-Foster 2005, Martin et al. 2012, Nazzi et al. 2012, Di Prisco et al. 2016). The deformed wing virus (DWV) occurs in Varroa-free bees and was present before the arrival of the mite (Wilfert et al. 2016). However, Varroa can transmit DWV in an injection-like manner by piercing into the bee's cuticle to feed on the bee's fat body and haemolymph (de Miranda and Genersch 2010, Ryabov et al. 2014, Ramsey et al. 2019). This new transmission route has likely driven the re-emergence of DWV (Wilfert et al. 2016). The devastating effects of Varroa and DWV quickly became apparent even before the mite had been established globally. Scientists were alarmed and able to study more recent Varroa invasions in 'real-time'. In Hawaii, which remained Varroa-free up until 2007, DWV strains diversity rapidly decreased and viral titres increased shortly after the mite was introduced (Martin et al. 2012). Changes in DWV strains and incidence rates were not limited to bees but also affected wild pollinators and associates within the host community (Santamaria et al. 2018, Loope et al. 2019). In New Zealand, Varroa infestations were first 
observed in northern parts of the North Island in 2000 (Mondet et al. 2014). The mite has since spread southwards, infesting bees in most areas of mainland New Zealand by 2013 (Mondet et al. 2014). Along this invasion front, titres of DWV, but also other viruses, increased shortly after mites were established (Mondet et al. 2014).

The global invasion of Varroa shows how the human-mediated movement of animals can drastically alter viral infection dynamics. Understanding the dynamics of infectious diseases and how pathogens circulate within complex multi-host communities is necessary to mitigate disease threats (Cleaveland et al. 2001). Genetic methods and phylogenetic analyses provide useful tools to infer pathogen transmission, reconstruct epidemiological history and understand environmental factors influencing disease spread (Archie et al. 2009, FountainJones et al. 2018).

\subsection{Species invasions can alter disease dynamics}

Infectious disease dynamics are shaped by host and pathogen communities (FountainJones et al. 2018), but humans are altering these communities by introducing hosts or pathogens into new regions (Daszak et al. 2000). New sea, land, and air links have created new and efficient pathways for the introduction of new species (Hulme 2009). Human-mediated species introductions can break down biogeographic barriers and thereby drive land transformation and biodiversity losses (Vitousek et al. 1997, Capinha et al. 2015). Emerging and re-emerging pathogens are often opportunists that respond to changes in host or pathogen ecologies (Dobson and Foufopoulos 2001). Species introductions bring a risk of altering these dynamics, which could lead to disease emergence or enhancement (Faillace et al. 2017). Humans have unknowingly altered viral dynamics in pollinator communities for centuries (Vanbergen et al. 2018). Commercially produced bumble bees in particular carry many bee-infecting pathogens, including RNA viruses and have, for instance, introduced microsporidian and neogregarine parasites into South American pollinator communities (Arbetman et al. 2013, Graystock et al. 2013, Schmid-Hempel et al. 2014). 
Many economically important pollinator species are social insects (e.g. A. mellifera and Bombus spp.), however, this group also harbours some of the most damaging invasive species. The highly organised colonies of social insects in which labour division ensures efficient foraging, brood care, and defence mechanisms are likely contributing to social insects becoming the dominant species in many ecosystems (Wilson 1971). These same traits have also equipped social insects to be successful invaders of many habitats (Moller 1996). Ants are particularly successful invaders (Holway et al. 2002, Bertelsmeier et al. 2017). The International Union for Conservation of Nature lists four ant species among their list of 100 of the worst invasive species worldwide (Lowe et al. 2000). New social behaviours such as polygyny (multiple queens) and unicoloniality in ants or large perennial colonies in wasps have arisen in some of these species' introduced ranges (Hölldobler and Wilson 1977, Wilson et al. 2009). This plasticity in breeding structure is tightly linked to the invasion success of many ant and wasp species (Eyer and Vargo 2021).

\subsection{Invasive Argentine ants as a threat to honey bees}

The Argentine ant (Linepithema humile) is one of these social insect species that is a successful invader. Argentine ants are native to South America but now occur throughout the world in Mediterranean and subtropical climates (Suarez et al. 2001, Roura-Pascual et al. 2004). Distribution models predict that Argentine ants will further expand their range, especially with global warming of higher latitude areas (Hartley and Lester 2003, Roura-Pascual et al. 2004, Roura-Pascual et al. 2011). Argentine ants are aggressive predators and competitors that can become extremely abundant in their introduced range, displace native arthropod species (Lach 2007, Cooling et al. 2015), and even act as nest predators for birds (Suarez et al. 2005). Argentine ants can also be a competitor with honey bees for nectar (Buys 1987). For instance, honey bees may avoid ant-occupied flowers (Lach 2007, Sidhu and Wilson Rankin 2016). Viruses can be transmitted between species through shared environments and the detection of bee pathogens on flowers suggests that many species are exposed to pathogens through foraging on shared flowers (McArt et al. 2014, Adler et al. 2018, Figueroa et al. 2019). Argentine ants were found positive for four viruses common in honey bees (Sebastien et al. 2015, Gruber et 
al. 2017, Payne et al. 2020). Like most ants, Argentine ants do not provide pollination services, yet, they are common flower visitors and therefore exposed to pollinator pathogens.

Argentine ants were first found in New Zealand in 1990 in Auckland (Green 1990) and have since become widespread in many locations in the North Island, but can also be found in several locations of the South Island as far south as Christchurch (Charles et al. 2002, Ward et al. 2010). Argentine ants can disturb honey production and pollination services provided by honey bees by preying on bees and robbing beehives (Harris 2002). Particularly in the Northland region of New Zealand, Argentine ants have been reported to attack bees, raid their hives, and contribute to hive losses (Gruber et al. 2017, Brenton-Rule 2018, Stahlmann-Brown et al. 2020). Sebastien et al. (2015) first found evidence for replication of a bee-infecting virus (DWV) in Argentine ants and noted that the species could provide a reservoir for bee-infecting viruses. A recent study on beehives with Argentine ants showed high mortality rates when bees were attacked by ants and an increase in DWV (Brenton-Rule 2018). Whether Argentine ants can vector viruses and contribute to the epidemic of bee-infecting viruses has not been studied yet.

\subsection{Harnessing viral pathogens to improve Argentine ant control in apiaries}

Controlling Argentine ants in apiaries could help prevent hive losses (Brenton-Rule 2018). Apiculture is a booming industry in New Zealand with export prices for honey steadily increasing (Ministry of Primary Industries 2018). The European honey bee was introduced to New Zealand from England in 1839 (Donovan 2007) and numbers of managed colonies are increasingly growing since; hive numbers have almost doubled between 2012 and 2017 (Ministry of Primary Industries 2018). There is a risk that non-native bees may be inefficient pollinators of New Zealand native plants and facilitate the spread of introduced plants (Huryn and Moller 1995, Huryn 1997), nevertheless, honey bees are needed to pollinate introduced crops and pasture. 
Overwinter colony losses in New Zealand are on average below $10 \%$, which is lower than losses in Europe and North America (Brown et al. 2018, Stahlmann-Brown et al. 2020). However, overall annual losses are increasing (Stahlmann-Brown et al. 2020). Argentine ants are listed as a cause for $5 \%$ of the colony losses that occur in New Zealand in the upper North Island (Brown et al. 2018). Varroa and the use of miticides to manage mite populations have increased the cost of honey bee pollination services (Ministry of Primary Industries 2018). Hive mortality and loss of apiary sites due to Argentine ants, the further spread of Argentine ants and controlling Argentine ants in apiaries provides another, potentially costly challenge.

Conventional Argentine ant control using pesticides often fails to achieve long-term population reductions or eradications (Hoffmann et al. 2016), likely because strategies are not adapted to account for the unicolonial population structure of invasive ants (Silverman and Brightwell 2008). Yet, their social system may also provide a mechanism by which pesticide transfer within colonies and increased secondary mortality can be achieved (Buczkowski and Wossler 2019). Effective insecticides or biological controls that do not harm non-target organisms such as bees are needed (Silverman and Brightwell 2008). Pathogens could be a contributing factor in the population collapses that have been observed in some invasive ant species (Simberloff and Gibbons 2004, Lester and Gruber 2016). Population collapses of Argentine ants have also occurred in New Zealand (Cooling et al. 2012) and provide hope for the presence of a pathogen that could be harnessed for population control. Although it is unknown whether bee-infecting RNA viruses cause disease in Argentine ants (Baty et al. 2020, Payne et al. 2020), several viruses have been discovered in this ant species (Gruber et al. 2017, Viljakainen et al. 2018) that could be more closely associated with ants and affect ant health.

RNA interference (RNAi) could become a species-specific and environmentallyfriendly tool for pest control (Baum et al. 2007, Whyard et al. 2009, Lopez et al. 2019) and may even be linked to biological control using pathogens. Through a conserved cellular gene regulation mechanism, specific genes can be silenced in targeted species by applying sequencespecific double-stranded RNA (dsRNA) (Hannon 2002). With decreasing dsRNA costs, this method could soon become widely available for pest control (Burand and Hunter 2013, Mat 
Jalaluddin et al. 2019). RNA interference has successfully been used to treat viral disease and Varroa parasitisation in honey bees (Hunter et al. 2010, Leonard et al. 2020). It is possible that silencing immune-related genes using RNAi could reduce immune function and lead to pathogen emergence, thereby reducing ant numbers.

\subsection{Thesis outline and aims}

The main aim of this research was to test whether Argentine ant presence can affect viral pathogens in bees. I aimed to examine disease dynamics within pollinator and arthropod communities that include Argentine ants and honey bees. I studied interactions between Argentine ants and honey bees in apiaries with the goal to test whether pathogens and beehive survival are affected by interactions between the two species. I further explored the feasibility of a novel ant control method that could provide a cost-effective long-term strategy to control populations and limit damage from ants.

In Chapter 2, I investigated the host range of RNA viruses in arthropod communities that include Argentine ants and honey bees. Many viruses that can be found in honey bees have been detected in wild bee species and other arthropods. I examined arthropods for viruses and viral replication, which is important to contribute to the understanding of disease dynamics. Using publicly available viral sequences from GenBank and viral sequences from New Zealand arthropods, I constructed viral phylogenies and tested whether viral strains were associated with host species or geographic regions.

In Chapter 3, I used a longitudinal field study to find out how Argentine ants affect pathogen infections and mortality in honey bees. Over several months, I compared beehives with and without Argentine ants and screened beehives as well as ant nests for infections with bee-associated viruses to determine whether ants affect bee health. 
Chapter 4 further investigated how Argentine ants affect viral infections in honey bees. Using RNA sequencing (RNA-seq), I studied the diversity of RNA viruses in hives and tested whether viral infections and transcriptomic responses in bees that interact with Argentine ants were different from those that do not.

In Chapter 5, I explored the feasibility of using double-stranded RNA (dsRNA) for Argentine ant control in apiaries. RNA interference could potentially be used to silence immune function in ants. If this immune reduction leads to the re-emergence of a pathogen that is already present within populations, it could help reduce ant numbers. While the development of RNAi for pest control is desirable to reduce the use of pesticides, testing its effectiveness and delivery are key in developing successful pest control. I tested how this novel approach compares to a conventional pesticide and how controlling Argentine ant populations affects beehives.

In Chapter 6, I discuss the general findings and implications of this work. I highlight fundamental research questions that are essential for managing disease in honey bees and explore avenues for future research and the development of Argentine ant control. 


\section{Chapter 2:Genetic strain diversity of multi-host RNA viruses that infect a wide range of pollinators and associates is shaped by geographic origins}

\subsection{Abstract}

Emerging viruses have caused concerns about pollinator population declines, as multihost RNA viruses may pose a health threat to pollinators and associated arthropods. In order to understand the ecology and impact these viruses have, we studied their host range and determined to what extent host and spatial variation affect strain diversity. Firstly, we used reverse transcription PCR to screen pollinators and associates, including honey bees (Apis mellifera) and invasive Argentine ants (Linepithema humile), for virus presence and replication. We tested for the black queen cell virus (BQCV), deformed wing virus (DWV), and Kashmir bee virus $(\mathrm{KBV})$ that were initially detected in bees, and the two recently discovered Linepithema humile bunya-like virus 1 (LhuBLV1) and Moku virus (MKV). Deformed wing virus, $\mathrm{KBV}$, and $\mathrm{MKV}$ were detected and replicated in a wide range of hosts and commonly coinfected hymenopterans. Secondly, we placed KBV and DWV in a global phylogeny with sequences from various countries and hosts to determine the association of geographic origin and host with shared ancestry. Both phylogenies showed strong geographic rather than hostspecific clustering, suggesting frequent inter-species viral transmission. Transmission routes between hosts are largely unknown. Nonetheless, avoiding the introduction of non-native species and diseased pollinators appears important to limit spillovers and disease emergence. 


\subsection{Introduction}

Pollinator communities worldwide are declining at an alarming rate (Potts et al. 2010, Hallmann et al. 2017, Sánchez-Bayo and Wyckhuys 2019). Emerging viral pathogens have been considered to be major contributors to pollinator losses alongside other drivers, such as habitat destruction; increased use of pesticides and fertilizers; climate change; and biological factors, such as invasive species (Potts et al. 2010, Goulson et al. 2015, Sánchez-Bayo and Wyckhuys 2019). The disruption of pollination services could cause severe effects on modern agriculture and biodiversity (Gallai et al. 2009, Manley et al. 2015). Insect pollination accounts for an economic value of over $€ 150$ billion annually, and without insect pollinators, food security would be at risk (Gallai et al. 2009). While managed honey bees and bumble bees are increasingly used to supplement crop pollination, these species cannot replace pollination by wild insects (Garibaldi et al. 2013).

Viral infections have been extensively studied in the European honey bee (Apis mellifera) because a number of RNA viruses that are circulating within bee populations have been associated with reduced health and reports of colony collapses (Evans and Schwarz 2011, McMenamin and Genersch 2015). Increasing evidence suggests that many of these pathogens are not specific to honey bees and instead are shared between many pollinator species and associated arthropods, including bumble bees and other wild bees (Singh et al. 2010, Evison et al. 2012, Murray et al. 2019); bee predators, such as wasps (Singh et al. 2010, Evison et al. 2012); and scavenging insects, such as ants, cockroaches, and beetles (Singh et al. 2010, Levitt et al. 2013, Sebastien et al. 2015, Schläppi et al. 2019). Many of these host species have been found cohabiting in beehives (Levitt et al. 2013, Sebastien et al. 2015, Brettell et al. 2019) or share floral resources with honey bees (Fürst et al. 2014, Bailes et al. 2018, Santamaria et al. 2018, Alger et al. 2019). Although arthropods harbour an enormous diversity of viruses (Li et al. 2015, Shi et al. 2016), little is known about the host range and dynamics of viruses within pollinator communities. 
Between-host viral transmission plays a key role in virus epidemics (Woolhouse et al. 2005). Emerging infectious diseases are commonly caused by pathogens that infect and interact through multiple host species (Johnson et al. 2015). Emerging RNA viruses are suspected to spill from honey bees into associates and vice versa (Fürst et al. 2014, Manley et al. 2015, Tehel et al. 2016, Alger et al. 2019). Due to high mutation rates and short generation times, this class of viruses is likely to infect and adapt to new host species and spread through large populations (Holmes 2009, Singh et al. 2010). Thus far, transmission routes between species are poorly understood; flowers have been suggested to represent "disease transmission hubs" (Figueroa et al. 2019), allowing for transmission without direct interactions among species. Closely related viral strains in several pollinators (Singh et al. 2010) and clustering of virus strains within geographic regions (Levitt et al. 2013, Fürst et al. 2014, Murray et al. 2019) indicate that ongoing inter-species viral transmission occurs. In order to improve pollinator conservation, multi-host viruses need to be studied further and outside the Apis genus (Manley et al. 2015).

Species introductions can alter virus communities through the introduction of new pathogens and though introduced hosts providing reservoirs for existing pathogens (Faillace et al. 2017). For instance, the global spread of the parasitic mite and bee virus vector, Varroa destructor, affected virus spread and infection levels in honey bees (Martin et al. 2012) but also caused pathogen shifts in other pollinators and bee predators, such as wasps (Santamaria et al. 2018, Loope et al. 2019). Invasive ants with supercolonial social structures in which many interconnected nests form a cooperative network are suspected to tolerate high pathogen loads and form disease reservoirs (Cremer 2019). In New Zealand, the invasive Argentine ant (Linepithema humile) has been hypothesized to form such a reservoir for honey bee viruses (Sebastien et al. 2015). Three bee viruses have been identified in this ant species (Sebastien et al. 2015, Gruber et al. 2017) and frequent interactions with honey bees through ants robbing honey or brood (Buys 1990) may facilitate viral transmission.

In this study, we tested the hypothesis that a range of pollinators and associated arthropods, including honey bees and Argentine ants, are infected by the same viral pathogens. Furthermore, we hypothesised that frequent inter-species viral transmission results in viral 
phylogenies that are predominantly associated with geographic origin instead of being associated with host species. Firstly, we screened pollinators and associated arthropods for five RNA viruses and tested them for virus replication. Most bee-affecting viruses are positive-sense single-stranded RNA (+ssRNA) viruses (Galbraith et al. 2018, Grozinger and Flenniken 2019), including deformed wing virus (DWV), Kashmir bee virus (KBV), black queen cell virus (BQCV), and Moku virus (MKV). However, we also tested for a negative sense ssRNA (-ssRNA) virus that was recently discovered in Argentine ants, the Linepithema humile bunya-like virus 1 (LhuBLV1). Limited knowledge about the host range of these viruses, particularly hosts of 'bee viruses' outside bees, makes identifying hosts and potential reservoirs an important step in understanding virus emergence. Secondly, we quantified to what degree the host species and geographic origin of DWV and KBV strains correlate with shared ancestry. We used phylogenetic analysis and phylogeny-trait correlation of our sequences supplemented with viral sequences retrieved from GenBank from various hosts all over the world. Phylogenies separated by geographic regions with hosts interspersed within these can provide evidence for inter-specific transmission. Alternatively, clustering within hosts species could indicate a high degree of host specificity.

\subsection{Materials and methods}

\subsubsection{Specimen collections}

We used honey bees (A. mellifera) and Argentine ants (L. humile) as two focal species for this study and sampled these species more frequently than other arthropod species. Adult workers were directly collected from ant nests $(n=32)$ and beehives $(n=51)$ using mouth aspirators or collections jars, respectively. Samples of pollinators and associated arthropods $(n=66)$ were collected using sweep nets or jars when directly collected from abandoned beehives. Collection sites consisted of a mix of agricultural fields, beach edge vegetation strips, and urban areas in the Northland region of New Zealand (Figure S2.1 and Figure S2.2). A total of 149 samples were collected in April $2018(n=135)$ and January $2019(n=14)$, some in apiaries, some near Argentine ant nests, and others without an apiary or ant nest nearby (details in Figure S2.2). Specimens were immediately snap-frozen in the field at approximately $-150{ }^{\circ} \mathrm{C}$ 
in a liquid nitrogen dry shipper, before being stored at $-80{ }^{\circ} \mathrm{C}$ in the laboratory until processing. Once in the laboratory, samples were removed from the freezer and placed on ice for identification. When possible, samples were identified to the species level and then again moved to $-80{ }^{\circ} \mathrm{C}$ until RNA extraction.

\subsubsection{RNA extraction and reverse transcription PCR for virus detection}

Samples were screened for four +ssRNA viruses, DWV, BQCV, KBV, and MKV, and an-ssRNA virus, LhuBLV1, using reverse transcription polymerase chain reaction (RT-PCR). MKV has only recently been discovered in wasps and bees (Mordecai et al. 2016b), and LhuBLV1 in Argentine ants (Viljakainen et al. 2018). A preliminary screening of a pooled sample for the seven Argentine ant viruses discovered by Viljakainen et al. (Viljakainen et al. 2018) concluded that only the -ssRNA virus LhuBLV1 was hosted by insects other than Argentine ants and was therefore included in the analysis. Individual whole arthropod samples were homogenized in $1000 \mu \mathrm{L}$ (ants in $500 \mu \mathrm{L}$ ) Trizol ${ }^{\mathrm{TM}}$ (Life Technologies, California, USA) and RNA was extracted and precipitated using isopropanol following the "Trizol"TM extraction" protocol as recommended in the COLOSS bee book (Evans et al. 2013).

RNA was quantified using a NanoPhotometer ${ }^{\circledR}$ (NP80, Implen, Germany) and approximately $500 \mathrm{ng}$ of RNA were treated with perfecta DNase (Quanta BioSciences, Maryland, USA) before reverse transcription to complementary DNA (cDNA) using qScript (Quanta BioSciences, Maryland, USA) following the manufacturer's instructions. PCR was carried out with MyTaq ${ }^{\mathrm{TM}}$ Mix and MyTaq ${ }^{\mathrm{TM}}$ Red Mix (Bioline, Meridian Bioscience Inc., UK). Here, $15-\mu \mathrm{L}$ reaction mixes consisted of $1 \mu \mathrm{L}$ of the template (1:20 diluted cDNA) and $0.4 \mu \mathrm{M}$ of each primer. PCR cycling conditions were $1 \mathrm{~min}$ at $95^{\circ} \mathrm{C}$, followed by 35 cycles $(38$ for $\mathrm{KBV}$ ) of $15 \mathrm{~s}$ of $95{ }^{\circ} \mathrm{C}, 15 \mathrm{~s} 55^{\circ} \mathrm{C}, 30 \mathrm{~s} 72{ }^{\circ} \mathrm{C}$, and a final $7 \mathrm{~min}$ of $72{ }^{\circ} \mathrm{C}$. Positive and negative controls were included in every PCR. Primer sequences used in this study can be found in the Supplementary Material (Table S2.1).

RT-PCR products were visualised on a $2 \%$ agarose gel (1.5\% for DWV) stained with SYBR ${ }^{\text {TM }}$ Safe DNA stain (Invitrogen, Life Technologies, California, USA) and run alongside 
a TrackIt 100-bp DNA ladder (Invitrogen, Life Technologies, California, USA). A subset of the resultant PCR amplicons was cleaned using ExoSAP-IT ${ }^{\mathrm{TM}}$ (Applied Biosystems ${ }^{\mathrm{TM}}$, Massachusetts, USA) following the manufacturer's instructions and sent for Sanger sequencing to Macrogen Inc (Seoul, South Korea). This subset included at least one virus-positive sample for every arthropod species for each virus. Sequence identity was confirmed using a BLASTn search (https://blast.ncbi.nlm.nih.gov/Blast.cgi) against the NCBI nucleotide collection and sequences were submitted to GenBank under the accession numbers MT068447 to MT068476.

\subsubsection{Negative strand detection}

DWV, KBV, MKV, and LhuBLV1-positive samples were further analysed for active viral infection. Strand-specific RT-PCR allows for detection of the negative strand, which in +ssRNA viruses (DWV, KBV, MKV) indicates active replication and parasitism of host cells by the virus (Craggs et al. 2001, Yue and Genersch 2005). Similarly, the positive strand in an -ssRNA virus (LhuBLV1) functions as mRNA for protein production or as a template to produce new -ssRNA and indicates an active infection (Guu et al. 2012). Equal amounts of RNA from virus-positive samples within each species were pooled and tested for negative or positive strand presence.

Reverse transcription reactions were carried out in the presence of a tagged primer that contains a non-viral sequence (tag) at the 5' end of the virus-specific primer (Yue and Genersch 2005). The non-viral sequence is then used in the PCR step in combination with a downstream virus-specific primer, so that only cDNA derived from the strand-specific tagged primer is amplified. Super-Script ${ }^{\mathrm{TM}}$ IV First-Strand Synthesis System (Invitrogen, Life Technologies, California, USA) was used to reverse transcribe $500 \mathrm{ng}$ of RNA into cDNA following the recommended COLOSS bee book protocol for strand-specific RT-PCR (de Miranda et al. 2015). To avoid the detection of false positives, the remaining RNA and tagged cDNA primers were digested using RNase H (Invitrogen, Life Technologies, California, USA) and $10 \mathrm{U}$ Exonuclease-I (Thermo Scientific ${ }^{\mathrm{TM}}$, Massachusetts, USA) at $37{ }^{\circ} \mathrm{C}$ for $30 \mathrm{~min}$ followed by inactivation at $70{ }^{\circ} \mathrm{C}$ for $15 \mathrm{~min}$ prior to PCR reactions, as described in the COLOSS bee book (de Miranda et al. 2015). PCR was carried out using tag and reverse primer in a $15-\mu \mathrm{L}$ reaction 
using myTaq ${ }^{\mathrm{TM}}$ Red Mix (Bioline, Meridian Bioscience Inc., UK). Cycling was 1 min at $95^{\circ} \mathrm{C}$, 30 cycles of $15 \mathrm{~s}$ at $95^{\circ} \mathrm{C}, 10 \mathrm{~s}$ at $55^{\circ} \mathrm{C}, 10$ to $30 \mathrm{~s} 72{ }^{\circ} \mathrm{C}$ (depending on product length), and a final $5 \mathrm{~min}$ of $72{ }^{\circ} \mathrm{C}$. The low cycling number was used to reduce the occurrence of unspecific bands. PCR products were visualised on an agarose gel and sequenced to confirm products as described above.

\subsubsection{Statistical analyses}

Statistical analyses were performed in R v3.4.1 (R Core Team 2014). Virus prevalence with $95 \%$ confidence intervals in the two focal species was determined using the epiR package (Stevenson et al. 2013) with 95\% sensitivity and specificity. Using the prop.test() function within R (R Core Team 2014), we tested if proportions of infected individuals differed between Argentine ants and honey bees.

\subsubsection{Phylogenetic analyses}

To conduct an analysis of globally occurring viral variants in different host species, we supplemented DWV and KBV sequences generated in this study with sequences obtained from GenBank (www.ncbi.nlm.nih.gov/genbank/). We used the same genomic regions that were used in the virus detection assay to conduct the phylogenetic analyses, which were a fragment of the $R N A$-dependent $R N A$ polymerase (RdRp) gene for DWV and the major capsid protein $v p 3$ for KBV. Through a BLASTn search, we retrieved 160 sequences that matched the DWV $R d R p$ fragment. Only DWV-A was included in the analysis because all DWV sequences from our sample in New Zealand matched the DWV-A group. For KBV, 11 sequences matching the $v p 3$ fragment were found and added to the sequences from this study. Most DWV and KBV sequences available on GenBank are from A. mellifera hosts; therefore, we tested an additional 34 Argentine ant samples obtained by Felden et al. (2019) from Argentina, California, and France for DWV and KBV (details in Table S2.2 and Felden et al. (2019)). Briefly, RNA was extracted from single ants using the Direct-zol RNA Microprep extraction kit (Zymo Research, California, USA) following the manufacturer's instructions. Approximately 50 ng of RNA were reverse transcribed using the High-Capacity cDNA Reverse Transcription kit (Applied 
Biosystems $^{\mathrm{TM}}$, Lithuania), tested for DWV and $\mathrm{KBV}$, and sequenced using the methods described above. From these samples, two DWV and nine KBV sequences were obtained and included in the analysis.

Sequences were trimmed and aligned using the ClustalW algorithm (Thompson et al. 1994) in Geneious v11.1.5 (Kearse et al. 2012). MEGA v.10.1 (Kumar et al. 2018) was used to find the best substitution model using Bayesian information criterion (BIC) scores, determining $\mathrm{HKY}+\mathrm{G}$ as the best model for both viruses. Final alignments were $440 \mathrm{bp}$ in the $R d R p$ region for DWV and $360 \mathrm{bp}$ in the $v p 3$ region for KBV.

Dated Bayesian phylogenetic analyses were performed in BEAST 2.2.6 (Bouckaert et al. 2014). Divergence times were calculated based on a tip-dated coalescent model. Clade probabilities were obtained from the posterior distribution incorporation sampling year information (retrieved from GenBank or original publications) for terminal nodes. Data were run under an uncorrelated lognormal relaxed clock model with gamma-distributed rate heterogeneity and coalescent tree prior with exponential population growth. Bayesian analyses were replicated three times and combined, each with Markov chain Monte Carlo of 150 million generations. Trees were sampled every 10,000 generations, of which the first $10 \%$ were discarded as burn-in. Using TreeAnnotator v1.7.5, we constructed maximum clade credibility trees and used FigTree v1.4.4 to visualise trees, including high posterior probabilities over 0.6. Tip-dated phylogenies are commonly used to reconstruct RNA viral evolution (Drummond et al. 2003, Rieux and Balloux 2016); however, there was a chance that the sampling date and sampling region in our dataset would be connected. Therefore, we repeated the analysis without using the tip-date calibration and compared dated and non-dated phylogenies.

\subsubsection{Phylogeny-trait correlation}

Phylogeny-trait association analysis was used to test to which extent phenotypic traits of a viral strain, such as host species or geographic location, are correlated with shared ancestry (Parker et al. 2008). Association index (AI), parsimony score (PS) (Wang et al. 2001), and monophyletic clade (MC) scores of dated and non-dated phylogenies were computed using 
BaTS (Bayesian tip-association significance testing) beta build 2 (Parker et al. 2008) by comparing a null model of random trait-tip assignments to the known trait distribution. BaTS accounts for phylogenetic uncertainty by testing many trees from the posterior distribution. Trait-tip association analyses were run with 500 replicates (500 random trees from the dated Bayesian phylogenetic analysis) to estimate a null distribution for each statistic.

\subsection{Results}

\subsubsection{Viral prevalence in pollinators and associated arthropods}

We collected arthropods from eight different orders. Virus-positive species were found in all except the Odonata, but we note that only two samples were taken from this order. Overall, viral infections were common in pollinators and associates, with $83 \%$ (95\% confidence intervals (CI): $76 \%-88 \%$ ) of samples testing positive for at least one virus and $44 \%(95 \% \mathrm{CI}$ : $36 \%-53 \%)$ having multiple infections. The majority of samples were hymenopterans $(n=105)$, out of which 97\% (95\% CI: 92\%-99\%) were infected with at least one and many with multiple $(62 \%, 95 \%$ CI: $52 \%-73 \%)$ viruses. One individual had as many as four viruses. Outside the order Hymenoptera $(n=46), 51 \%(95 \%$ CI: 37\%-65\%) of samples were found positive for at least one virus but only $6 \%(95 \% \mathrm{CI}: 2 \%-17 \%)$ for multiple (two) viruses.

\subsubsection{Viral coinfections in honey bees and Argentine ants}

Out of the five viruses tested, up to four were found to coinfect individual workers of Argentine ants and honey bees (Figure 2.1). The most prevalent viruses were DWV in honey bees (100\%, 95\% CI: 94\%-100\%) and LhuBLV1 in Argentine ants (78\%, 95\% CI: 61\%-90\%, Table 2.1). Linepithema humile bunya-like virus 1 was only detected in Argentine ants and associated arthropods in two sites that had ant nests, indicating that other species can host this virus, or that its detection in other arthropods could have resulted from contamination. Only Argentine ants tested positive for the mRNA intermediate of LhuBLV1, suggesting that this virus may be $L$. humile specific. Interestingly, all ants that tested positive for DWV were sampled from a nest in an apiary (Figure S2.2, site WAI). In a test of proportions, DWV was more prevalent in bees than in ants $\left(\mathrm{X}^{2}=35.98, p<0.001\right)$, KBV was more prevalent in ants 
than in bees $\left(\mathrm{X}^{2}=6.69, p=0.010\right)$, and MKV infections were not significantly different between the two species $\left(\mathrm{X}^{2}=0.15, p=0.689\right.$, Table 2.1$)$. Moku virus was found at a high prevalence in Vespinae species ( $n=17,88 \%$ infected, $95 \%$ CI: $65 \%-98 \%$ ); this group also showed a KBV prevalence similar to honey bees $(24 \%$, 95\% CI: $8 \%-49 \%)$.

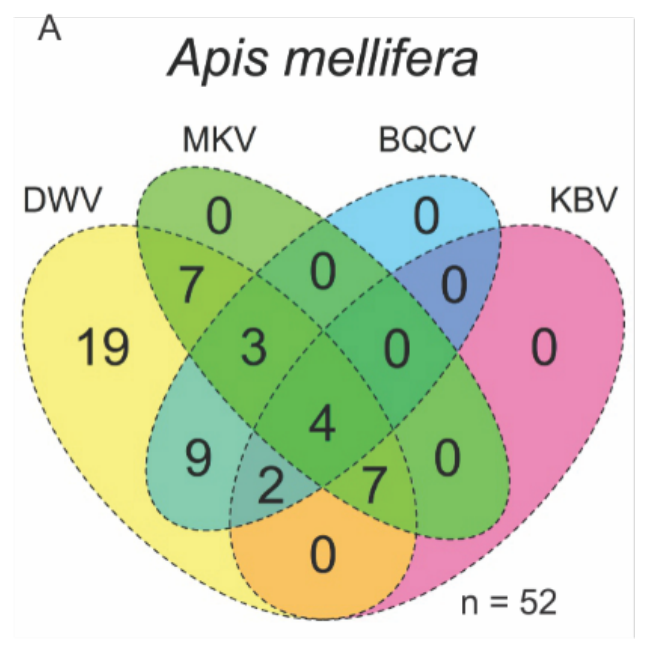

\section{${ }^{\mathrm{B}}$ Linepithema humile}

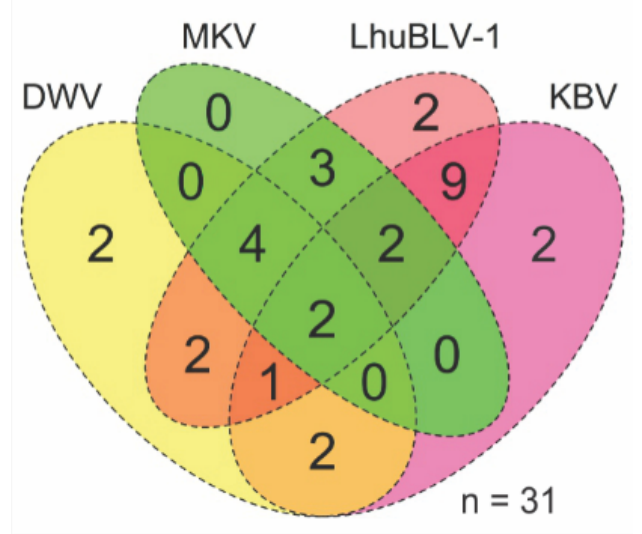

Figure 2.1: Venn diagram of viral coinfection in honey bees (A. mellifera) (A) and Argentine ants (L. humile) (B). Most prevalent viruses were DWV in honey bees and LhuBLV1 in Argentine ants. Virus abbreviations: BQCV: black queen cell virus; DWV: deformed wing virus; KBV: Kashmir bee virus; LhuBLV1: Linepithema humile bunya-like virus 1; MKV: Moku virus.

Table 2.1: Estimated viral prevalence in honey bees (A. mellifera) and Argentine ants $(L$. humile) for five virus targets with $95 \%$ confidence intervals (CI) in brackets. - indicates that the virus was not detected in the species and * indicates a significantly higher prevalence in a test of proportions. Virus abbreviations: BQCV: black queen cell virus; DWV: deformed wing virus; KBV: Kashmir bee virus; LhuBLV1: Linepithema humile bunya-like virus 1; MKV: Moku virus.

\begin{tabular}{llllll}
\hline Host & BQCV [\%] & DWV [\%] & KBV [\%] & LhuBLV1 [\%] & MKV [\%] \\
\hline \hline Apis mellifera & $35(23-49)$ & $100^{*}(94-100)$ & $25(15-39)$ & - & $41(28-55)$ \\
Linepithema humile & - & $41(24-58)$ & $56^{*}(39-72)$ & $78(61-90)$ & $34(20-53)$ \\
\hline \hline
\end{tabular}

\subsubsection{Viral replication within host species}

All viruses, except BQCV which was only detected in honey bees, were tested for virus replication, or active viral infections in the case of LhuBLV1. Active LhuBLV1 infection was only found in Argentine ants while all +ssRNA viruses replicated in multiple species (Table 2.2). Fourteen different species tested positive for DWV; active viral replication could be confirmed for five of them (Table 2.2). For KBV, 5 out of the 9 virus-positive species showed 
active replication and MKV replication was confirmed in 5 out of 10 species including the Polistes and Vespula species tested (Table 2.2). The negative-strand RT-PCR assay is highly conservative and can only confirm the presence of the negative strand intermediate, which is indicative of viral replication but does not allow the exclusion of the possibility for replication.

\subsubsection{Viral strain diversity and phylogenetic analyses}

Using BLAST searches, we confirmed the virus identity and identified locations and hosts infected with viral strains most similar to those observed in our samples. BLAST showed that BQCV most closely matched the polyprotein of BQCV found in a honey bee in Lithuania (KP223790, 96\% identity, 100\% query cover). The MKV sequences from 10 different species showed little sequence variation; all sequences derived in this study closely matched $(99 \%-$ $100 \%$ identity, $97 \%-100 \%$ query cover) the MKV polyprotein found in Vespa velutina in Belgium (MF346349) and Vespula pensylvanica from Hawaii (KU645789). Linepithema humile bunya-like virus 1 most closely matched the putative $R d R p$ complex gene of the only available LhuBLV1 sequences on GenBank (MH213237) from Argentine ants from Spain (100\% identity, 95\% query cover). 
Table 2.2: Viral presence in pollinators and associated arthropods tested for deformed wing virus (DWV), Kashmir bee virus (KBV), Moku virus (MKV), black queen cell virus (BQCV), and Linepithema humile bunya-like virus 1 (LhuBLV1) using RT-PCR. Virus not found in species: -; virus found in species: +; active viral replication confirmed: +/+. Summary in the bottom line.

\begin{tabular}{|c|c|c|c|c|c|c|c|c|c|c|}
\hline Order & Family & Genus & Species & Common name & $\mathrm{n}$ & DWV & KBV & MKV & BQCV & $\begin{array}{l}\text { LhuBL } \\
\text { V1 }\end{array}$ \\
\hline \multirow[t]{4}{*}{ Araneae } & Salticidae & Helpis & Helpis minitabunda & jumping spider & 1 & + & - & + & - & + \\
\hline & Lycosidae & Lycosa & 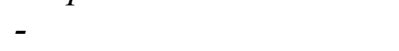 & wolf spider & 1 & - & - & - & - & - \\
\hline & Theridiidae & Steatoda & Steatoda capensis & black cobweb spider & 3 & - & + & $+/+$ & - & - \\
\hline & Thomisidae & Diaea & & flower spider & 4 & - & - & - & - & - \\
\hline \multirow[t]{2}{*}{ Blattodea } & Blattidae & Celatoblatta & - & cockroach & 9 & $+/+$ & + & + & - & - \\
\hline & & Maoriblatta & - & cockroach & 7 & + & - & + & - & + \\
\hline \multirow{4}{*}{$\begin{array}{l}\text { Coleoptera } \\
\text { Diptera }\end{array}$} & Curculionidae & Scolopterus & Scolopterus penicillatus & black spined weevil & 1 & + & - & - & - & - \\
\hline & Sarcophagidae & Jantia & Jantia crassipalpis & flesh fly & 2 & - & - & - & - & - \\
\hline & Stratiomyidae & - & - & solider fly & 2 & - & - & - & - & + \\
\hline & Tipulidae & Leptotarsus & - & crane fly & 2 & + & - & - & - & - \\
\hline \multirow{10}{*}{$\begin{array}{l}\text { Dermaptera } \\
\text { Hymenoptera }\end{array}$} & Forficulidae & Forficula & Forficula auricularia & European earwig & 2 & + & + & - & - & - \\
\hline & Apidae & Apis & Apis mellifera & honey bee & 51 & $+/+$ & $+/+$ & + & + & - \\
\hline & & Bombus & - & bumble bee & 2 & - & $+/+$ & - & - & - \\
\hline & Formicidae & Linepithema & Linepithema humile & Argentine ant & 32 & $+/+$ & $+/+$ & $+/+$ & - & $+/+$ \\
\hline & Pompilidae & Sphictostethus & Sphictostethus nitidus & golden hunter wasp & 1 & + & - & - & - & - \\
\hline & Vespidae & Polistes & Polistes chinensis & Chinese paper wasp & 11 & + & - & $+/+$ & - & - \\
\hline & & & Polistes humilis & Australian paper wasp & 2 & - & - & + & - & - \\
\hline & & Vespula & Vespula germanica & German wasp & 1 & $+/+$ & + & $+/+$ & - & - \\
\hline & & & Vespula vulgaris & common wasp & 3 & $+/+$ & $+/+$ & $+/+$ & - & - \\
\hline & Ichneumonidae & Xanthocryptus & $\begin{array}{l}\text { Xanthocryptus } \\
\text { novozealandicus }\end{array}$ & $\begin{array}{l}\text { lemon tree borer } \\
\text { parasite }\end{array}$ & 1 & + & - & - & - & - \\
\hline \multirow{4}{*}{$\begin{array}{l}\text { Odonata } \\
\text { Orthoptera }\end{array}$} & Lestidae & Austrolestes & Austrolestes colensonis & blue damselfly & 2 & - & - & - & - & - \\
\hline & Acrididae & Locusta & Locusta migratoria & migratory locust & 1 & - & - & - & - & - \\
\hline & Gryllidae & Bobilla & - & small field cricket & 6 & + & $+/+$ & - & - & + \\
\hline & & Teleogryllus & Teleogryllus commodus & black field cricket & 4 & - & - & - & - & - \\
\hline \multicolumn{2}{|c|}{$\begin{array}{l}\text { Total virus-positive species } \\
\text { (total samples) }\end{array}$} & & & & $\begin{array}{l}21 \\
(151)\end{array}$ & $\begin{array}{l}14 \\
(83) \\
\end{array}$ & $\begin{array}{l}9 \\
(44) \\
\end{array}$ & $\begin{array}{l}10 \\
(53) \\
\end{array}$ & $\begin{array}{l}1 \\
(18)\end{array}$ & $\begin{array}{l}5 \\
(32) \\
\end{array}$ \\
\hline
\end{tabular}


The phylogenetic analysis showed that DWV sequences found in New Zealand in this study clustered within the DWV-A clade and formed a monophyletic group with a sequence from A. mellifera from New Zealand (MF623172) and Vespa carbo from Italy (KY909333) (posterior probability $(\mathrm{PP})=0.539$, Figure 2.2). This group is dated to the year $2013(95 \%$ highest posterior density $(H P D)=2010-2016)$. However, when not including the sampling date in the phylogeny, sequences derived in this study clustered with a broader group of European DWV sequences (Figure S2.3). Two DWV strains from Pakistan (KP734706, KP734705) represented a relatively old clade $(\mathrm{PP}=1)$ that was estimated to have separated from other DWV sequences around 1977 (95\% HPD = 1965-1989, Figure 2.2). The DWV phylogeny further split into two sister groups $(\mathrm{PP}=0.95$, dated to $1986,95 \%$ HPD $=1979$ 1994), one from Asia and Europe, which includes the DWV-A variant Kakugo virus, and the other with strains from Europe, North and South America, and New Zealand (Figure 2.2 and Figure S2.3, non-dated phylogeny). Within this group, samples from New Zealand all occupy a different clade than samples from the Americas, including Hawaii, and both share these with Europe (PP $=1$, dated to $1999,95 \%$ HPD $=1998-2001$, Figure 2.2 and Figure S2, non-dated phylogeny). Interestingly the two strains collected from Australia (KP734699, KP734632) did not cluster together or with strains from New Zealand.

The phylogenetic analysis for KBV showed that the sister group of most New Zealand KBV sequences was a group from Tasmania ( $P P=0.641$, Figure 2.3 and Figure S2.4, nondated phylogeny). KBV from a New Zealand $V$. vulgaris, however, closely matched samples from the USA (HM228885, HM228887) ( $\mathrm{PP}=1$, Figure 2.3 and Figure S2.4, non-dated phylogeny). 


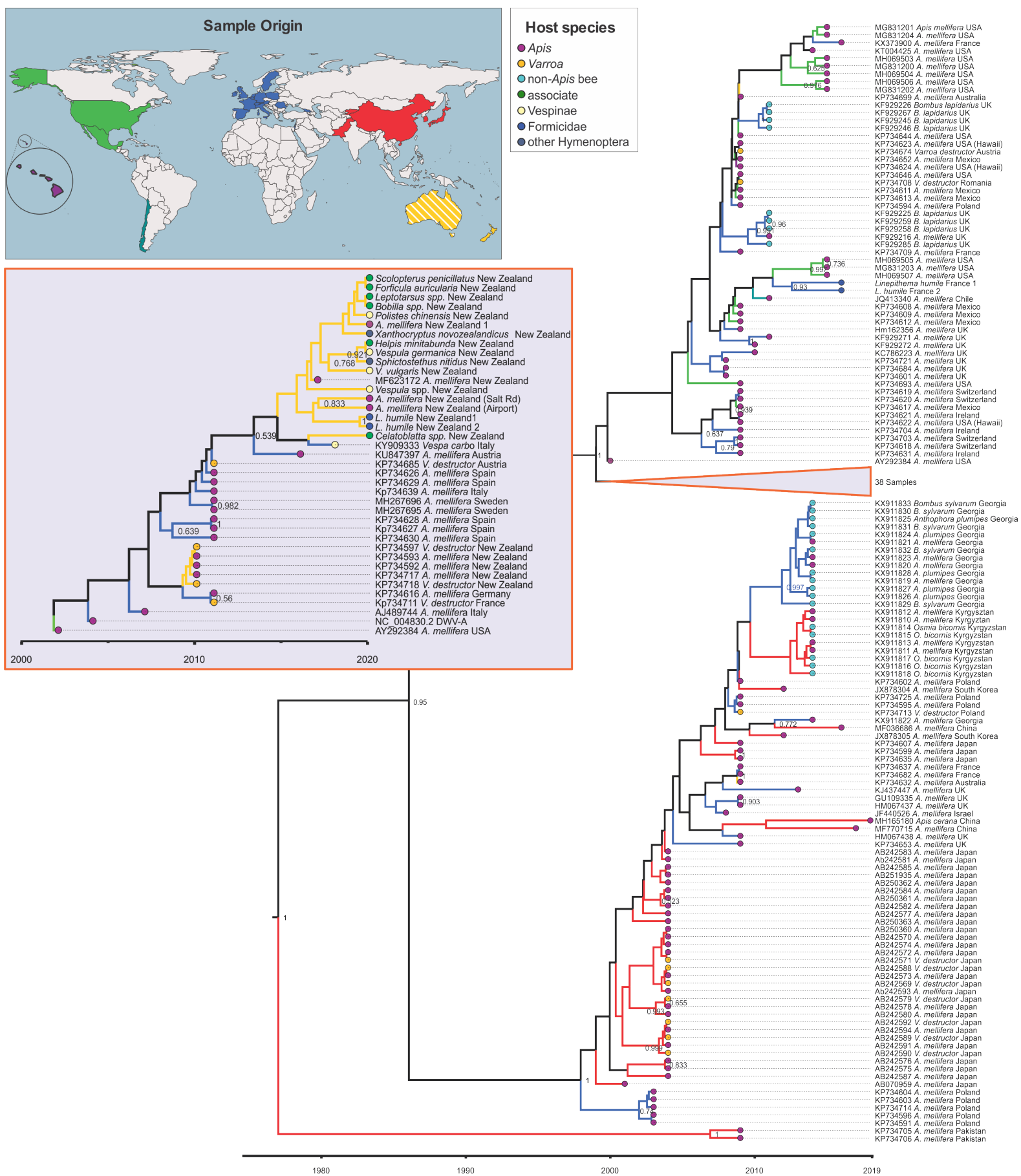

Figure 2.2: Maximum clade credibility tree for the $R N A$-dependent $R N A$ polymerase $(R d R p)$ fragment (440 bp) of deformed wing virus (DWV). Grey insert with orange frame shows the collapsed part of the tree that among others includes the 19 samples from New Zealand from this study. The species name, country of origin, and, if applicable, GenBank accession number are given in the branch label. The branches are coloured according to the lineages' inferred geographic origin as shown on the world map and end nodes are coloured according to the host group as shown in the host species insert. Posterior support $>0.6$ is given and the $\mathrm{x}$-axis shows time in years. 


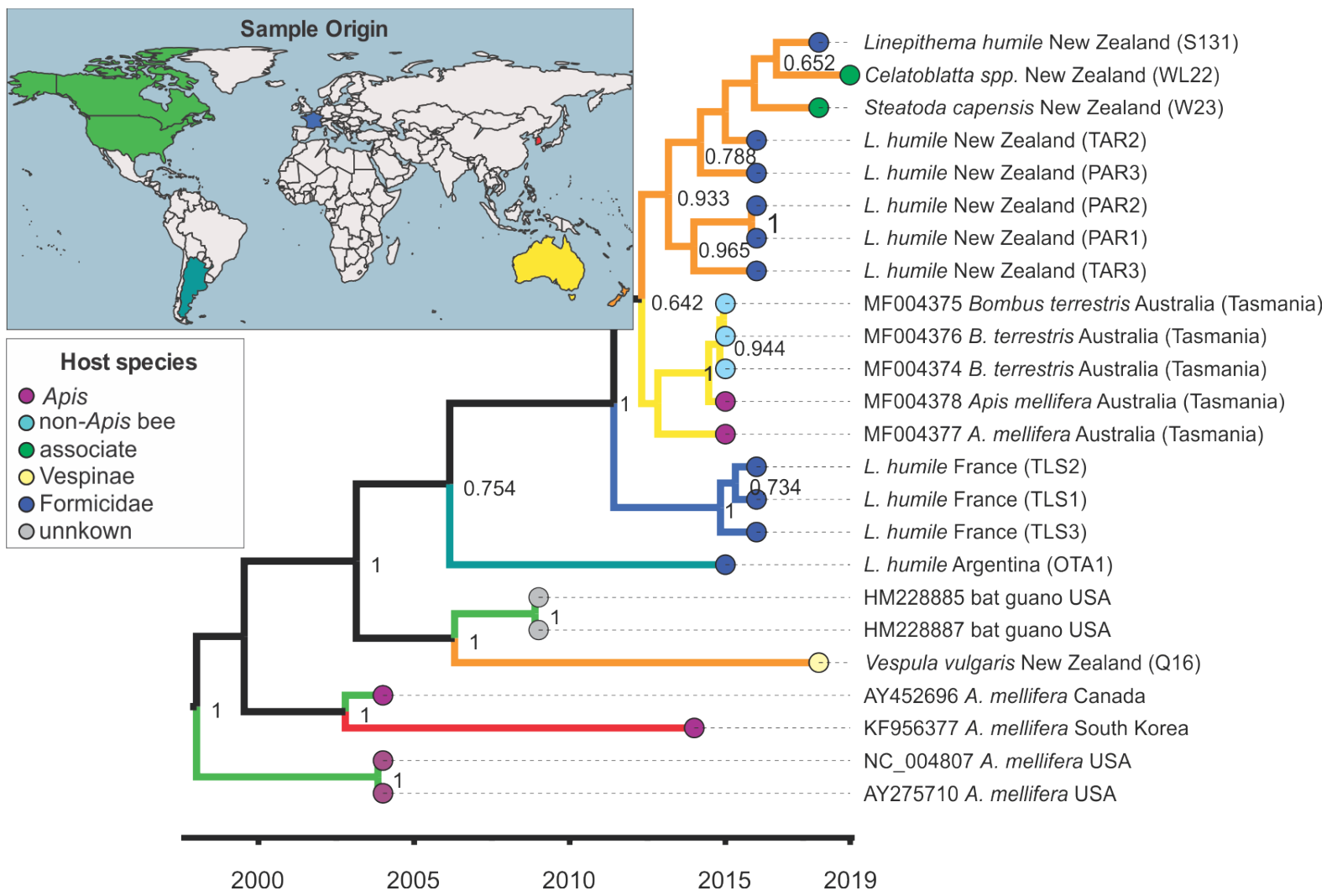

Figure 2.3: Maximum clade credibility tree for the major capsid protein $v p 3$ fragment $(360 \mathrm{bp})$ of Kashmir bee virus (KBV). The species name, country of origin, and, if applicable, GenBank accession number are given in the branch label. The branches are coloured according to the lineages' inferred geographic origin as shown on the world map and end nodes are coloured according to the host group as shown in the host species insert. Posterior support $>0.6$ is given and the $\mathrm{x}$-axis shows time in years.

\subsubsection{Trait-tip associations}

The trait-tip association analysis for DWV showed significant clustering within geographic locations and within host species in both dated and non-dated phylogenies (association index (AI) and parsimony score (PS), $p<0.01$, Table 2.3, and Table S2.3, traittip association in non-dated phylogeny). The PS, which indicates the number of state changes on a tree, was smaller for geographic locations than for host species (Table 2.3 and Table S2.3). Overall, observed to expected ratios indicated a stronger association with geographic origin than with host species (Table 2.3, Table S2.3). The maximum monophyletic clade (MC) index tests which traits are associated with phylogeny, and showed that the maximum observed clade size for Asia, Europe, North America, and Oceania was larger than expected by chance (all MC scores $p<0.01$, Table 2.3 and Table S2.3). The association was also statistically supported (all MC scores $p<0.05$ ) for a number of host states that included Apis, Varroa, bee associates, non-Apis bees, and L. humile (Table 2.3 , bee associates $p=1$ in non-dated analysis, Table 
S2.3), which indicates that these groups carry strains that are more closely related than expected due to chance. Yet, it is difficult to determine whether a geographic state or host state causes the association as the geographic origin and host species were often linked. For example, all North American samples in the analysis were from $A$. mellifera hosts and all bee associates were collected in New Zealand. Therefore, host and geographic origin can both contribute to an observed association.

Table 2.3: Trait-tip association in deformed wing virus (DWV), as calculated with 500 random trees (replicates) in BaTS (Bayesian tip-association significance testing) (Parker et al. 2008). Significant $p$-values are in bold font. A low parsimony score (PS) represents fewer state changes on the tree and a stronger trait-tip association. Maximum clade (MC) scores show the maximum size of a clade for a trait state; a high MC is positively correlated with trait-tip association. The geographic region of South America was only represented by one sample and is not shown.

\begin{tabular}{llllll} 
Statistic & $\mathrm{n}$ & $\begin{array}{l}\text { Observed to } \\
\text { expected ratio } \\
(95 \% \mathrm{CI})\end{array}$ & $\begin{array}{l}\text { Observed mean } \\
(95 \% \mathrm{CI})\end{array}$ & $\begin{array}{l}\text { Null mean } \\
(95 \% \mathrm{CI})\end{array}$ & $\begin{array}{c}p \\
\text { value }\end{array}$ \\
\hline \hline Association Index (AI) & & & & \\
\hline $\begin{array}{l}\text { Geographic } \\
\text { location }\end{array}$ & - & $0.21(0.13-0.30)$ & $2.83(1.95-3.74)$ & $13.43(12.47-14.47)$ & $<\mathbf{0 . 0 1}$ \\
\hline Host species & - & $0.55(0.40-0.74)$ & $4.96(3.89-6.01)$ & $8.98(8.17-9.76)$ & $<\mathbf{0 . 0 1}$ \\
\hline Parsimony Score (PS) & & & & \\
\hline $\begin{array}{l}\text { Geographic } \\
\text { location }\end{array}$ & - & $0.35(0.30-0.40)$ & $30.91(28.00-34.00)$ & $88.56(84.43-92.45)$ & $<\mathbf{0 . 0 1}$ \\
\hline Host species & - & $0.68(0.61-0.74)$ & $34.87(32.00-37.00)$ & $51.30(49.75-52.35)$ & $<\mathbf{0 . 0 1}$ \\
\hline Maximum Clade (MC) scores & 50 & - & & & $<$ \\
\hline Asia & 76 & - & $15.59(11.00-21.00)$ & $2.57(2.12-3.27)$ & $<\mathbf{0 . 0 1}$ \\
\hline Europe & 24 & - & $14.44(14.00-16.00)$ & $3.53(2.89-4.79)$ & $<\mathbf{0 . 0 1}$ \\
\hline North America & 25 & - & $4.48(3.00-8.00)$ & $1.66(1.28-2.09)$ & $<\mathbf{0 . 0 1}$ \\
\hline Oceania & 3 & - & $12.54(6.00-18.00)$ & $1.72(1.29-2.24)$ & $<\mathbf{0 . 0 1}$ \\
\hline Hawaii & 126 & - & $1.07(1.00-2.00)$ & $1.01(1.00-1.06)$ & 1 \\
\hline $\begin{array}{l}\text { Apis } \\
\text { Varroa destructor }\end{array}$ & 13 & - & $13.24(9.00-21.00)$ & $7.04(5.37-9.93)$ & $\mathbf{0 . 0 1}$ \\
\hline Associate & 6 & - & $1.70(1.00-3.00)$ & $1.27(1.01-1.99)$ & $\mathbf{0 . 0 4}$ \\
\hline Non-Apis bee & 23 & - & $1.83(1.00-3.00)$ & $1.05(1.00-1.19)$ & $<\mathbf{0 . 0 1}$ \\
\hline Vespidae & 5 & - & $4.23(4.00-5.00)$ & $1.63(1.23-2.07)$ & $<\mathbf{0 . 0 1}$ \\
\hline Formicidae & 4 & - & $1.09(1.00-2.00)$ & $1.03(1.00-1.15)$ & 1 \\
\hline Other & 2 & - & $2.00(2.00-2.00)$ & $1.02(1.00-1.07)$ & $<\mathbf{0 . 0 1}$ \\
\hline Hymenoptera & 2 & $1.00(1.00-1.00)$ & $1.00(1.00-1.00)$ & 1 \\
\hline \hline
\end{tabular}

For KBV, significant clustering within geographic regions and host species was found (AI and PS, $p<0.01$, Table 2.4 and Table S2.4, trait-tip association in non-dated phylogeny). Again, a stronger association with geographic origin was observed than with host species (observed to expected ratio, Table 2.4). Due to the limited number of available KBV sequences 
covering the $v p 3$ region $(n=24)$, samples from a geographic location were often sourced from the same host, and vice versa. KBV sequences significantly clustered within New Zealand, Europe, and Australia (all MC: $p<0.05$ ) but also within bumble bees (MC: $p<0.01$ ) (Table 2.4 and Table S2.4). The 10 L. humile samples that were positive for KBV did not cluster together (MC: $p=0.16$, Table 2.4) and instead, sequences appeared to be divided by geographic region.

Table 2.4: Trait-tip associations for Kashmir bee virus (KBV), as calculated with 500 random trees (replicates) in BaTS (Bayesian tip-association significance testing) (Parker et al. 2008). Significant $p$-values are in bold font. A low parsimony score represents fewer state changes on the tree and a stronger trait-tip association. Maximum clade scores show the maximum size of a clade for a trait state; a high MC is positively correlated with trait-tip association. The geographic regions of Asia and South America were only represented by one sample each and are not shown.

\begin{tabular}{|c|c|c|c|c|c|}
\hline Statistic & $\mathrm{n}$ & $\begin{array}{l}\text { Observed to } \\
\text { expected ratio } \\
(95 \% \mathrm{CI})\end{array}$ & $\begin{array}{l}\text { Observed mean } \\
(95 \% \mathrm{CI})\end{array}$ & $\begin{array}{l}\text { Null mean } \\
(95 \% \mathrm{CI})\end{array}$ & $p$-value \\
\hline \multicolumn{6}{|c|}{ Association Index (AI) } \\
\hline \multicolumn{5}{|l|}{ Geographic } & $<0.01$ \\
\hline Host species & & $0.27(0.11-0.50)$ & $0.58(0.29-0.79)$ & $2.14(1.59-2.57)$ & $<0.01$ \\
\hline \multicolumn{6}{|c|}{ Parsimony Score (PS) } \\
\hline $\begin{array}{l}\text { Geographic } \\
\text { location }\end{array}$ & & $0.45(0.40-0.58)$ & $6.06(6.00-7.00)$ & $13.44(12.12-14.92)$ & $<0.01$ \\
\hline Host species & & $0.59(0.50-0.73)$ & $7.55(7.00-8.00)$ & $12.74(11.00-13.98)$ & $<\mathbf{0 . 0 1}$ \\
\hline \multicolumn{6}{|c|}{ Maximum Clade (MC) scores } \\
\hline North America & 4 & - & $2.00(2.00-2.00)$ & $1.26(1.00-2.00)$ & 0.10 \\
\hline New Zealand & 9 & - & $7.83(6.00-7.00)$ & $1.92(1.07-3.00)$ & $\mathbf{0 . 0 1}$ \\
\hline Europe & 3 & - & $3.00(3.00-3.00)$ & $1.10(1.00-2.00)$ & 0.01 \\
\hline Australia & 5 & - & $4.37(4.00-5.00)$ & $1.24(1.00-2.00)$ & 0.01 \\
\hline Apis & 6 & - & $2.54(2.00-4.00)$ & $1.45(1.00-2.94)$ & 0.19 \\
\hline Bombus & 3 & - & $2.97(2.00-3.00)$ & $1.10(1.00-2.00)$ & $<0.01$ \\
\hline Linepithema & 10 & - & $3.08(3.00-4.00)$ & $2.12(1.10-3.44)$ & 0.16 \\
\hline associate & 2 & - & $1.09(1.00-2.00)$ & $1.03(1.00-1.17)$ & 1 \\
\hline
\end{tabular}

\subsection{Discussion}

Our study shows that multiple RNA viruses that were initially detected in honey bees or Argentine ants infect a range of pollinators and associated arthropods. We have added to the growing body of evidence of bee viruses infecting and replicating in non-Apis hosts. To our knowledge, this work represents the first study to confirm active LhuBLV1 and MKV infections in arthropod hosts by detecting the positive and negative viral strand intermediates, respectively. Virus replication in novel hosts is a key contributor to disease emergence; 
nevertheless, even hosts without active virus replication may show active virus replication at a different time (Boncristiani et al. 2009) or can be contaminated with infectious viral particles that contribute to disease spread (Manley et al. 2015). The arthropods we examined typically did not display any symptoms of viral infection. The absence of obvious symptoms is common in dicistroviruses like BQCV and KBV (Bonning and Miller 2010), yet there may be negative effects on the host. For example, covert KBV infections in bumble bees reduce reproduction (Meeus et al. 2014) and DWV-infected honey bees rarely display wing deformities, although covert infections reduce foraging activity and long-term survival (Fürst et al. 2014, Benaets et al. 2017). To which extent these viruses affect hosts other than bees is not well characterised and remains to be investigated (Manley et al. 2015).

\subsubsection{Host range of RNA viruses}

DWV appears to be the most prevalent honey bee pathogen, occurring in approximately 55\% of colonies worldwide (Martin and Brettell 2019). We found high DWV prevalence in honey bees but also in Argentine ants. The list of DWV hosts is long (Martin and Brettell 2019). We add to it by reporting the first DWV case in the order Orthoptera (Bobilla) and New Zealand native Hymenoptera (Xanthocryptus novozealandicus and Sphictostethus nitidus), Coleoptera (Scolopterus penicillatus), and Blattodea (Maoriblatta).

In the case of the more recently discovered viruses, such as MKV and LhuBLV1, the knowledge of disease symptoms, dynamics, or host range is very limited (Mordecai et al. 2016b, Viljakainen et al. 2018). Studies suggest that the predatory wasp $V$. pensylvanica may represent an MKV reservoir on Hawaii (Mordecai et al. 2016b, Brettell et al. 2019). We confirmed active MKV replication in three different wasp species and an overall high prevalence in Vespinae, supporting the potential virus reservoir. Negative-sense ssRNA viruses of the Bunyavirales family are found in many species, including LhuBLV1 in Argentine ants (Viljakainen et al. 2018) and Apis mellifera Bunyavirus 1 and 2 in honey bees (Remnant et al. 2017). We detected the positive strand intermediate of LhuBLV1 in Argentine ants but not in any other host. LhuBLV1 did not seem specific to Argentine ants, as four other species were found virus-positive. Yet, no pollinator species tested positive for this virus, making LhuBLV1 unlikely to be an emerging virus that poses a risk on pollinator communities. 


\subsubsection{Pathogen reservoir and viral spillover into wild populations}

Pathogen transmission may potentially alter competition among multiple host species, so that spatial interactions are important when regarding transmission networks (Seabloom et al. 2015). RNA viruses initially identified in honey bees can be widespread in the environment (Figueroa et al. 2019), but only some novel hosts acquire infections. High densities of apiaries or introduced ants, such as the Argentine ant, could allow accumulation of high viral levels and possible spillover into pollinators and associates that would account for the virus-positive species found in this study. Frequently, the main drivers for disease emergence in wildlife populations are spillovers from domesticated hosts and human-mediated pathogen invasion, also termed 'pathogen pollution' (Daszak et al. 2001). Introduced pathogens can provide a competitive benefit to introduced hosts if native hosts are more susceptible to the pathogen than the invader (Faillace et al. 2017). Moreover, domestic animals often outnumber wild species and can act as reservoir hosts that may drive the population decline of the wild host (Daszak et al. 2001). For instance, the introduction of rabies virus into the Serengeti ecosystem through domestic dogs caused spillovers into wild carnivore communities and ongoing efforts to vaccinate dogs are required to stop the disease spread (Lembo et al. 2008). In case of the European honey bee, Varroa-mediated high DWV titres in bees may cause spillovers and promote virus spread to associates and other pollinators (Wilfert et al. 2016). The presence of apiaries has been shown to cause a high prevalence of DWV and BQCV in wild pollinators (Alger et al. 2019) and species associated with beehives (Singh et al. 2010, Levitt et al. 2013, Ravoet et al. 2014). We found BQCV in honey bees but no other pollinators, which may be due to the higher BQCV prevalence in honey bees compared to other bees (Manley et al. 2020), and the low number of wild bee pollinators in our sample set. We only detected DWV in Argentine ants from apiaries, which could indicate that ants acquire the virus from bees, potentially by scavenging in apiaries. In addition to species introduction, anthropogenic actions reducing biodiversity in landscapes and species may facilitate disease transmission and outbreaks (Dobson 2004).

\subsubsection{Coinfections and interactions among viruses}

Coinfections and interactions between viral species within the host can play a key factor in the epidemiology and evolution of viruses (Alizon et al. 2013). Many insects that we analysed tested positive for multiple viruses. Interactions within hosts and, for example, the order in which pathogens are acquired may have a large positive or negative effect on a 
secondary infection (Telfer et al. 2010, Natsopoulou et al. 2017). In some cases, infection with one virus can prevent infection with another: consequently, this makes the infection beneficial if it is less damaging or virulent than the other (Mordecai et al. 2016a, Viljakainen et al. 2018). Multiple parasites may compete for resources or even release toxins that inhibit the growth of a competitor (Mideo 2009, Natsopoulou et al. 2015). These interactions affect adaptive responses to the infection and dynamics within the community (Lello et al. 2004, Mideo 2009). Thereby interactions can be strain-specific and affect parasite evolution and genetic diversity within virus populations (Seppälä et al. 2009). Future work is needed to understand coinfections and interactions among viruses in addition to the commonly studied host-parasite interactions (Telfer et al. 2010).

\subsubsection{Multi-host viruses in emerging disease}

Single-stranded RNA viruses have been considered to be the most likely type of pathogen to jump between species and cause disease outbreaks (Woolhouse et al. 2005) that could devastate wild pollinator populations. High mutation rates in RNA viruses result in virus populations with high variation amongst genotypes (Holmes 2009). Over time, multi-host parasites can evolve into different species-specialist strains or populations, or remain a generalist parasite with lower than optimal virulence (Rigaud et al. 2010). For multi-host viruses, a lack of host specificity may result in a trade-off between virulence in different hosts that depends on the host quality and availability (Rigaud et al. 2010). Whether a virus that invades a new host causes an emerging disease or only a minor outbreak depends on factors such as its ability to replicate in a novel host and transmission routes (Woolhouse et al. 2005, Manley et al. 2015).

We confirmed DWV, KBV, and MKV replication in several species, indicating that these species possibly act as biological vectors that facilitate disease emergence. However, with pollinators and associates collected in the field, we cannot exclude the possibility of detecting viruses and negative-strand intermediates in the gut contents that come from other infected insects. Kashmir bee virus and MKV replication were confirmed in species not typically known to eat bees, such as Polistes wasps and bumble bees. Some cockroaches, crickets, wasps, and ants that tested positive for virus replication were found close to beehives and are species known to scavenge or prey on honey bees (Buys 1990, Loope et al. 2019). Recently consumed viral particles from infected honey bees can cause false positives in replication assays. For instance, Varroa mites that exclusively feed on honey bee tissue (Ramsey et al. 2019) and vector DWV are assumed to act as a biological vector that propagates DWV (Yue and Genersch 
2005, Gisder et al. 2009). Yet, new research suggests that DWV may not replicate in mite cells but honey bee cells recently consumed by mites (Posada-Florez et al. 2019). Nevertheless, feeding experiments have shown that scavenger species like the ant Myrmica rubra are found positive for the negative strand of DWV for up to 13 weeks after consuming infected honey bees (Schläppi et al. 2019), which indicates that DWV actively infects ants. Whether honey bee scavengers can spread viruses without becoming infected themselves (Porter et al. 2016) or aid in reducing viral transmission by removing infectious carcasses (Le Sage et al. 2019) remains to be tested. The detection of negative-strand intermediates is an essential step in identifying host species, but only controlled infection experiments allow the determination of the virus dynamics in these hosts and the potential for disease emergence.

\subsubsection{Global distribution and evolution of bee viruses}

To understand the current epidemic of bee viruses, it is key to determine the role of the host species and geographic distribution (Murray et al. 2019, Manley et al. 2020). By placing the sequences generated in this study within a global phylogeny, we found that both KBV and DWV showed strong grouping by geographic regions. Closely related viral strains in geographic locations support the hypothesis that viral infections can be acquired from the environment and other host species in the same location (Murray et al. 2019). Although DWV was present in New Zealand before the introduction of Varroa in 2001 (Mondet et al. 2014), all New Zealand DWV strains grouped within the clade that emerged in the early 2000s. The DWV phylogeny showed some broad separations into geographic regions, and only European samples were scattered across groups. Whether European strains are found across the phylogeny because introduced honey bees or other virus-carrying arthropods from Europe initiated the spread remains to be tested further. Research indicates that the global DWV epidemic has been mediated by European $A$. mellifera populations but that the virus shows little host specificity (Wilfert et al. 2016). Although the KBV phylogeny only represents a limited number of the true KBV diversity, sequences from Argentine ants showed a weak association with the phylogeny, suggesting that ants acquired local KBV strains. Moreover, the high prevalence of KBV in this species could indicate a disease reservoir, and ants facilitating further spreading of KBV. 


\subsubsection{Conclusions}

Many emerging viruses infect and actively replicate within a wide range of pollinators and associated arthropod species. Our results and others indicate that the host species can affect the association with shared ancestry, which indicates viral transmission, but that these effects are weaker than the geographic location (Levitt et al. 2013). Overall, geographic locations played a major role in shaping patterns of viral genetic diversity. As viruses may be frequently transmitted between species, it is important to incorporate the host abundance and diversity, spatial structure of communities, and multi-host systems into studies of pathogen dynamics and epidemics (Seabloom et al. 2015). Avoiding the introduction of non-native species and transport of diseased pollinators is important to stop disease spillovers and prevent disease emergence. 


\section{Chapter 3:Invasive ants are associated with elevated deformed wing virus infections in honey bees}

\subsection{Abstract}

Emerging viral pathogens and suspected frequent inter-species viral transmission have sparked concerns about pollinator health. Several RNA viruses that infect the European honey bee (Apis mellifera) can be found in a wide range of pollinating insects and hive pests. Yet, how interactions with other species affect viral dynamics is poorly understood. The globally invasive Argentine ant (Linepithema humile) is a common pest in honey bee apiaries and a known host of bee-associated viruses. To test whether Argentine ants affect viral infections in honey bees, we moved beehives into sites with and without Argentine ants and monitored hive survival and pathogen infections in both species over seven months. We found three common honey bee pathogens in Argentine ants: Deformed wing virus (DWV), Kashmir bee virus $(\mathrm{KBV})$ and black queen cell virus (BQCV). Although the presence of ants did not affect beehive mortality throughout our experiment, DWV and BQCV titres in bees were significantly affected by ant presence while the microsporidian Nosema ceranae and trypanosomatid Lotmaria passim were not. Deformed wing virus infection levels in bees without ants peaked in mid-autumn and decreased towards winter, while DWV in bees with Argentine ants showed significantly higher infection levels in mid-autumn and remained elevated until early winter. Whether ants affect viral infections in bees through transmitting viruses, either as a biological or mechanical vector, needs to be studied further. Even in the absence of inter-species viral transmission, invading and robbing ants may lead to stress that can facilitate viral disease. Our work shows that invasive ant presence is associated with changes in the seasonal dynamics of a key honey bee pathogen, DWV. Understanding how ants affect viral dynamics and honey bee health could have important implications for disease management. 


\subsection{Introduction}

Insect populations have been in decline in recent decades (Hallmann et al. 2017, van Klink et al. 2020). In particular, pollinator health decline and associated stressors that include habitat loss, monotonous diets, pesticides and pathogens (Potts et al. 2010, Goulson et al. 2015) may affect ecosystem services provided by pollinations, leading to reductions in crop yields and threats to food security (Goulson et al. 2015). Emerging viral diseases have gained increasing interest due to associations with honey bee mortality (Cox-Foster et al. 2007, Ratnieks and Carreck 2010) and their potential to affect wild pollinator communities (Fürst et al. 2014, Manley et al. 2015). Viral infections in honey bees may appear asymptomatic at first (Grozinger and Flenniken 2019) but are frequently associated with colony collapses (CoxFoster et al. 2007, Cornman et al. 2012, McMenamin and Genersch 2015).

Bee-infecting viruses have been found in many insects and other arthropod species that cohabit in beehives and interact with honey bees (Singh et al. 2010, Levitt et al. 2013, Brettell et al. 2019, Tapia-González et al. 2019, Dobelmann et al. 2020). Disease dynamics in such multi-host multi-pathogen systems are complex. Certain host species and even individual foraging preferences can disproportionally affect disease persistence (Kilpatrick et al. 2006, Ellner et al. 2020). Introductions of novel species may alter entire host-pathogen communities (Faillace et al. 2017). Invasive predators, for instance, can transform pollination systems through the consumption of pollinators (Vanbergen et al. 2018). The global spread of Varroa mites in honey bees demonstrates how the introduction of a vector transformed not only the virus communities in honey bees (Martin et al. 2012) but also in wild bees (Manley et al. 2019, Manley et al. 2020) and honey bee predators (Loope et al. 2019). These wide-ranging effects should raise awareness that inter-species interactions and novel vector species can affect virus dynamics in host communities. Inter-species viral transmission and spread to new host populations play a key role in the dynamics of emerging viral diseases (Woolhouse et al. 2005). How inter-species transmission affects viral communities in bees is an active area of investigation (Grozinger and Flenniken 2019).

Ants are exposed to viruses that are typically found within beehives through robbing honey and pollen stores, preying on bee brood or scavenging on dead bees (Schläppi et al. 2019, Payne et al. 2020, Schläppi et al. 2020, Yañez et al. 2020). Acute bee paralysis virus (ABPV), black queen cell virus (BQCV), deformed wing virus (DWV), Israeli acute paralysis virus (IAPV) and Kashmir bee virus (KBV) are five bee-infecting viruses that have been repeatedly detected in ant species (Celle et al. 2008, Levitt et al. 2013, Sebastien et al. 2015, 
Gruber et al. 2017, Brettell et al. 2019, Lester et al. 2019, Baty et al. 2020, Payne et al. 2020). Disease symptoms of bee-infecting viruses in ants are rarely studied (but see Schläppi et al. 2020) and the host status of ants is debated. Some studies found that the prevalence of beeassociated viruses in ants decreases outside of apiaries (Gruber et al. 2017, Payne et al. 2020). The suspected primary transmission route from bees to ants is foodborne through predation and robbing (Schläppi et al. 2019, Payne et al. 2020, Schläppi et al. 2020, Yañez et al. 2020). Whether vice versa viral transmission, from ants to bees, occurs has not been shown yet. It is possible that ants act as a vector or viral reservoir (Sebastien et al. 2015, Payne et al. 2020). Invasive ant populations often occur in high densities with low genetic variation and potentially harbour high pathogen loads (Holway et al. 2002, Cremer 2019) facilitated by increased withinspecies transmission rates in dense populations (Bailes et al. 2020).

The Argentine ant (Linepithema humile) is one of the most widespread and damaging invasive ant species (Holway et al. 2002). Argentine ants have been observed to attack bees (Buys 1990, Brenton-Rule 2018) and disrupt foraging activities when honey bees share flowers with Argentine ants (Sidhu and Wilson Rankin 2016). In New Zealand, Argentine ants were first observed in 1990 (Green 1990) and have rapidly extended their range since (Ward et al. 2010). Three honey bee-associated viruses (BQCV, DWV, and KBV) have been detected in Argentine ants in New Zealand \{Gruber, $2017 \# 1148$ \}. To test the hypothesis that Argentine ants can affect viral infections in honey bees, we used a controlled field experiment in which we moved hives of the European honey bee (Apis mellifera) into sites with and without Argentine ants. We monitored hive survival and pathogen infections in ants and bees over seven months.

\subsection{Material and methods}

\subsubsection{Experimental setup and sampling}

We selected 18 nucleus beehives that had been established four to five weeks before the start of the experiment by a commercial beekeeping operation (Haines Apiaries, Kaitaia, New Zealand). All hives were made up out of a single brood box with eight frames and a box for honey collection on top. In January 2019, we moved all 18 hives into six different sites in the Northland region of New Zealand, placing three hives in each site (Figure 3.1, Figure S3.1). Three sites had known Argentine ant incursions while the other three were Argentine ant-free. We confirmed this presence or absence of Argentine ants by placing baits consisting of cotton balls soaked in sugar water on the ground. We placed 20 baits in a four-by-five grid with each 
bait being approximately one metre apart from each other. After 30 minutes baits were checked for ant presence and we counted the number of ants at each bait. In sites without Argentine ants, we found low numbers of Ochetellus glaber and Nylanderia $s p$. and in sites with Argentine ants, no other ant species were found.

\section{A. Study sites}

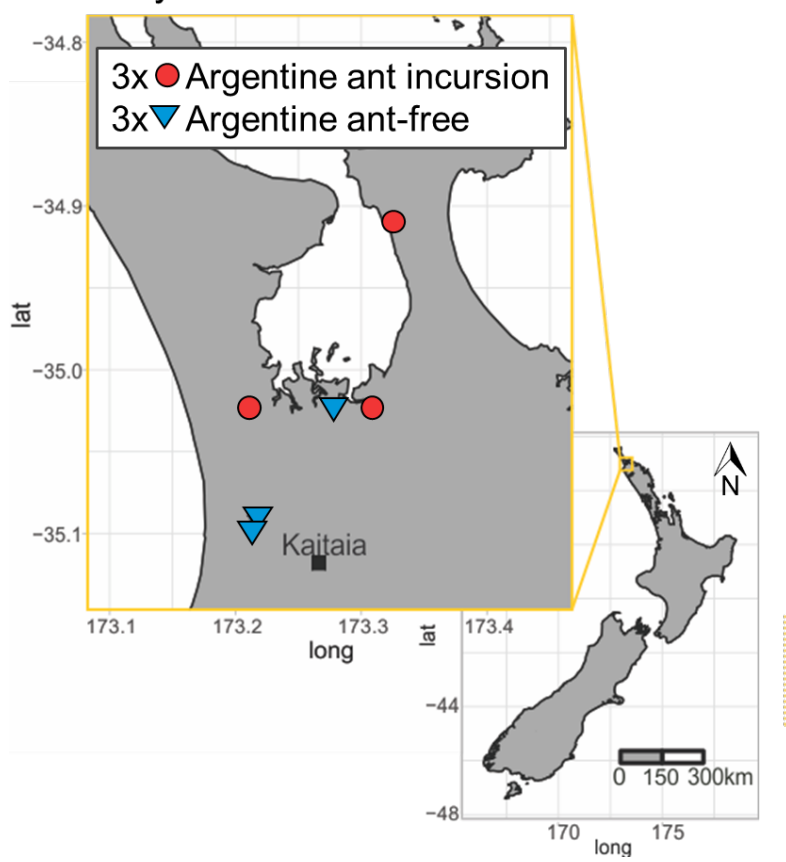

B. Treatments

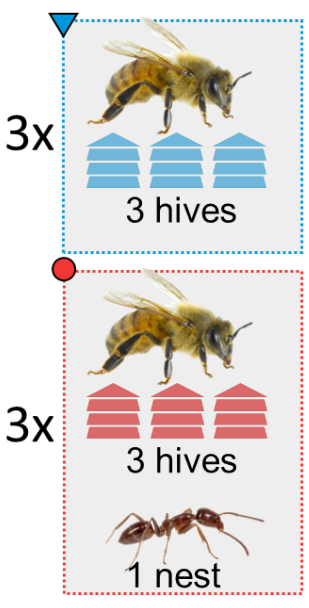

18 hives moved to 6 sites in January Data collection from January until August (7x)

Figure 3.1: Experimental design to test the effect of Argentine ants on viral infections in beehives. A: Three sites with known ant infestations and three sites without Argentine ants in the north of New Zealand were chosen for the study. B: Three beehives were moved into each of the six sites and monitored over seven months. C: Honey bees and Argentine ants were tested for bee-associated viruses: BQCV (black queen cell virus), DWV (deformed wing virus), KBV (Kashmir bee virus). Honey bees were additionally screened for two pathogens that have not been found in Argentine ants: N. ceranae and L. passim. ** Ants were screened for the beeassociated ABPV (acute bee paralysis virus), which was not detected.

The hives were monitored from January 2019 (summer) to August 2019 (winter) in monthly intervals, except July. Bayvarol® strips (Bayer New Zealand Limited, New Zealand) were used to control Varroa populations in June (sites HOW, WIL, Figure S3.1) or May (all other sites). To detect the presence of Varroa mites, a sugar shake was performed for every colony (Macedo et al. 2002). Briefly, honey bees from a frame containing capped brood were shaken into a bowl and approximately 300 bees were transferred into a jar containing icing sugar using a measuring cup. The jar was carefully rolled around to ensure all bees were coated in icing sugar. We left the jar for at least 3 min before shaking out the sugar into a bowl through a mesh lid. The sugar dust causes mites to dislodge from the bees' bodies and fall into the bowl (Macedo et al. 2002) where they were counted. Additionally, frames were visually inspected 
for the presence of bee eggs and the queen. Returning forager bees were counted for 1 min with three repetitions as an estimate for bee foraging activity. Adult A. mellifera were collected from frames containing brood for molecular analyses. In sites with L. humile incursions, ants were collected using an aspirator, when possible from foraging trails on the beehives. To avoid RNA degradation, samples were frozen immediately after collection in a liquid nitrogen dry-shipper at approximately $-150^{\circ} \mathrm{C}$ while in the field. A total of 97 honey bee samples and 21 Argentine ant samples were obtained from January until August 2019. In March, an additional four ant nests that were not in apiaries were sampled for a comparison between Argentine ants inside and outside apiaries.

\subsubsection{Homogenisation and RNA extraction}

Pools of 25 bees from each hive at each time point (except for January and February where 12 and 20 bees, respectively, were pooled) were washed with approximately $10 \mathrm{~mL}$ phosphate-buffered saline (PBS, Ambion, Texas, USA) to reduce the risk of contamination from pathogens on the cuticle (as used in Celle et al. 2008, Li et al. 2015, Shi et al. 2016). Bees were then placed in $7 \mathrm{~mL}$ homogeniser tubes with three $3.2 \mathrm{~mm}$ steel beads. Bees were homogenised in a bead mill homogeniser (Precellys Evolution, Bertin Technologies, France) at $4^{\circ} \mathrm{C}$ using three intervals of $30 \mathrm{~s}$ at $7500 \mathrm{rpm}$ with $5 \mathrm{~s}$ pauses in between. Immediately following the homogenisation, approximately $0.5 \mathrm{~g}$ of the homogenate was added to $600 \mu \mathrm{L}$ TRIzol ${ }^{\circledR}$ Reagent (Life Technologies, California, USA) and vortexed for 20 s. A $350 \mu \mathrm{L}$ volume of the homogenate-TRIzol mix was transferred into a spin column for RNA extraction. Extractions were carried out using the Direct-zol RNA Miniprep Plus Kit (Zymo Research, California, USA) that includes an in-column DNase treatment following the manufacturer's instructions. RNA was eluted in $100 \mu \mathrm{L}$ nuclease-free water, and quantity was measured using a NanoPhotometer ${ }^{\circledR}$ (NP80, Implen, Germany). One $\mu \mathrm{g}$ RNA was used for reverse transcription to complementary DNA (cDNA) in a $20 \mu \mathrm{L}$ reaction using the High-Capacity cDNA Reverse Transcription Kit (Applied Biosystems, Invitrogen, California, USA) according to the manufacturer's instructions. Reactions proceeded at $25^{\circ} \mathrm{C}$ for $10 \mathrm{~min}, 37^{\circ} \mathrm{C}$ for $120 \mathrm{~min}$ and $85^{\circ} \mathrm{C}$ for $5 \mathrm{~min}$. The cDNA was then diluted to $100 \mu \mathrm{L}(1: 5)$ before being used in downstream quantitative PCR (qPCR) reactions.

For each Argentine ant sample, 50 ants were counted while on dry ice and washed three times with $500 \mu \mathrm{L}$ PBS (Ambion, Texas, USA) using fresh PBS each time to reduce the chance of contamination with viruses from the cuticle. Samples were placed in $2 \mathrm{~mL}$ tubes and homogenised in $500 \mu$ L TRIzol ${ }^{\circledR}$ Reagent (Life Technologies, California, USA) in a bead mill 
homogeniser (Precellys Evolution, Bertin Technologies, France) with $0.5 \mathrm{~mm}$ steel beads at $4^{\circ} \mathrm{C}$ with two intervals of $20 \mathrm{~s}$ at $6800 \mathrm{rpm}$ and a $30 \mathrm{~s}$ pause. RNA was extracted using $450 \mu \mathrm{L}$ of the homogenate with the Direct-zol RNA Miniprep Plus Kit (Zymo Research, California, USA) including the DNase treatment following the manufacturer's instructions and eluted in $100 \mu \mathrm{L}$ nuclease-free water. RNA concentrations were analysed using a NanoPhotometer ${ }^{\circledR}$ (NP80, Implen, Germany). RNA (800 ng) was used in reverse transcription reactions using SuperScript IV (Invitrogen, Thermo Fisher Scientific, Lithuania) following the manufacturer's instructions.

\subsubsection{Pathogen detection and quantification in honey bees}

Firstly, we aimed to detect which bee pathogens were present in our sample. Endpoint PCR assays were used to screen pooled samples of bee RNA for 11 candidate pathogens. Bee samples were pooled within the six sampling sites and within collection seasons (January to February: summer; March to May: autumn; and June to August: winter). In this initial screening, we found DWV, BQCV, Lake Sinai virus 1 and 2, and Sacbrood virus in more than 13 out of the 18 pooled samples. Other pathogens with lower prevalences were KBV, chronic bee paralysis virus, and Moku virus (found in $<6$ sample pools). The microsporidian Nosema apis was not detected in any sample pools but $N$. ceranae was detected in 14 and the trypanosome Lotmaria passim was found in all samples. Although KBV was not common in bees, we chose to include the virus in further analyses as it had previously been found in Argentine ants along with BQCV and DWV (Gruber et al. 2017). Consequently, the pathogens we examined in honey bees in the qPCR analysis were $N$. ceranae and L. passim and the three bee-associated viruses BQCV, DWV, and KBV. Lotmaria passim and N. ceranae are not known to infect ants but provide an additional indicator of bee health and may interact with other stressors and pathogens.

Quantitative PCR for honey bee samples was carried out in $20 \mu \mathrm{L}$ reactions on a 48-well plate using a StepOne ${ }^{\mathrm{TM}}$ Real-Time PCR cycler (Applied Biosystems, California, USA). Reactions were run in duplicate using the PowerUp SYBR green master mix (Applied Biosystems, Lithuania) containing $4 \mu \mathrm{L}$ 1:5 diluted cDNA (approximately 40 ng cDNA) and $400 \mathrm{nM}$ of each primer. Apis mellifera actin related protein 1 (GenBank accession: NM_001185145) was included as an endogenous control to normalise pathogen titres between samples. Primer sequences can be found in Table S3.1. Negative controls were included in every mix to rule out non-specific amplification. Cycling was performed with an initial 2 min at $50^{\circ} \mathrm{C}$ and $2 \mathrm{~min}$ at $95^{\circ} \mathrm{C}$, followed by 40 cycles of $3 \mathrm{~s}$ at $95^{\circ} \mathrm{C}$ and $30 \mathrm{~s}$ at $60^{\circ} \mathrm{C}$. A melt curve 
analysis stage was included to confirm amplification of a single product with an increase from $60^{\circ} \mathrm{C}$ to $95^{\circ} \mathrm{C}$ measuring fluorescence at every $0.3^{\circ} \mathrm{C}$ increment. Raw qPCR data were analysed using the Thermo Fisher Connect Relative Quantification app v4.3 to obtain cycle quantification $\left(\mathrm{C}_{\mathrm{q}}\right)$ values, which were then exported to and analysed in $\mathrm{R} v 3.6 .3$ ( $\mathrm{R}$ Core Team 2014). Mean $C_{q}$ values and standard error (SE) were calculated from technical replicates and qPCR runs were repeated when the $\mathrm{SE}$ exceeded $0.5 \mathrm{C}_{\mathrm{q}}$. Cycle quantification values below 35 were regarded as positive detection to avoid false detection from late-cycle amplification. The pathogen load was normalised to reference gene levels $\left(\mathrm{C}_{\mathrm{q}}\right.$ actin $)$ and calculated as $2^{-\Delta \mathrm{Cq}}$.

\subsubsection{Pathogen detection and quantification in Argentine ants}

For Argentine ant samples, probe-based TaqMan chemistry was designed to detect ABPV, BQCV, KBV, DWV and eleven ant-specific viruses (Table S3.1). We did not include IAPV, because it is not found in New Zealand (McFadden et al. 2015), although it has been detected in ants elsewhere. The ant-specific viruses included Linepithema humile bunya-like virus 1 (LhuBLV1), Linepithema humile C-virus 1 (LhuCV1), Linepithema humile entomopoxvirus 1 (LhuEV1), Linepithema humile polycipivirus 1 (LhuPCV1), Linepithema humile polycipivirus 2 (LhuPCV2), Linepithema humile picorna-like virus 1 (LhuPiLV1), Linepithema humile partiti-like virus 1 (LhuPLV1), Linepithema humile rhabdo-like virus 1 (LhuRLV1), Linepithema humile qinvirus-like virus 1 (LhuQLV1), Linepithema humile totilike virus 1 (LhuTLV1), and Linepithema humile virus 1 (LHUV1). Dynactin subunit 4 (GenBank accession: LOC105671957) was used as the reference gene to normalise virus levels in ants across samples. Quantitative PCR using the 21 ant samples from the controlled experiment and the additional four samples collected from nests outside of apiaries was performed using TaqMan Array Micro Fluidic Cards (Applied Biosystems, ThermoFisher Scientific, California, USA). TaqMan Fast Advanced Master Mix (Applied Biosystems, ThermoFisher, USA) was mixed with $800 \mathrm{ng}$ cDNA for qPCR (final concentration of $2 \mathrm{ng} / \mu \mathrm{L}$ ). Thermal cycling on a QuantStudio 7 Real-Time PCR System (Applied Biosystems, ThermoFisher Scientific, USA) was conducted as per the manufacturer's instructions. Viral loads were calculated by normalising $\mathrm{C}_{\mathrm{q}}$ values to reference gene levels $\left(\mathrm{C}_{\mathrm{q}}\right.$ dynactin $)$ and calculated as $2^{-\Delta \mathrm{Cq}}$. 


\subsubsection{Statistical analyses}

Statistical analyses were performed in R v3.6.3 (R Core Team 2014). Data did not follow a normal distribution and could not be normalized using standard transformation techniques, therefore non-parametric tests were used. Spearman's rank correlation analysis was used to assess the correlation between the pathogens (except KBV as it was only found in 9.28 $\%$ of hives) and foraging activity within honey bee samples and correlations of individual viruses between bees and ants from the same apiary using the stats package in R. The effect of Argentine ant presence on hive survival was examined using a log-rank test and Kaplan-Meier survival curves were generated using the package survminer (Kassambara et al. 2017).

We compared individual pathogen loads and foraging activity between treatments using permutational multivariate analysis of variance (PERMANOVA) with the adonis() function in vegan in $\mathrm{R}$ (Oksanen et al. 2017). Pathogens that were not detected were treated as a pathogen load of zero. The pathogen load or the number of returning foragers was used as the response variable and treatment (ants vs. no ants), sampling time point, their interaction, and site nested within treatment as fixed effects. Analyses were run with 999 permutations using Manhattan distances. Permutations were restricted to sampling time points to account for repeatedly measuring the same hives. Kashmir bee virus was excluded from the analysis as it was only detected in beehives in sites with ants. Samples collected in January were regarded as a baseline measurement, before ant presence or absence could affect hives, and were therefore excluded from this analysis. 


\subsection{Results}

\subsubsection{Hive survival and foraging activity}

In two sites, Argentine ants were found foraging on beehives shortly after hives were moved into the site in January. However, in the third site (WAI in Figure S3.1) ants foraging on the hives were seen from March onwards. We observed ants robbing honey and pollen stored within beehives, ants attacking bee brood, and ants scavenging on dead adult bees. No Argentine ants were observed in the control sites throughout the experiment. Out of the 18 hives at the beginning of the experiment, only six survived until August (A in Figure 3.2). Two hives in sites with ants were found dead after only one month; on both, we observed large numbers of ants the day after hives were placed. Another hive in the ant-treatment group lost its queen possibly in February and no brood could be found in this hive until it was found dead in May. Overall, mortality did not differ between hives exposed to ants and hives without ants (Log-rank test: $p=0.88$, A in Figure 3.2). Varroa mites were rarely detected in beehives, and only ever found in four sites (three without and one with Argentine ants) at low infestation rates that did not exceed 1\% (maximum of two mites in approximately 300 bees). No Varroa mites were found in January or August, and April had the highest Varroa prevalence (25\%). Foraging activity differed significantly between treatments but also between sites and observation time points (PERMANOVA: $\mathrm{F}_{\text {Treatment }}=6.206, \mathrm{R}^{2}$ Treatment $=0.050, p_{\text {Treatment }}=0.016$; $\mathrm{F}_{\text {Site }}=2.701, \mathrm{R}^{2}$ Site $=0.086, p_{\text {Site }}=0.027 ; \mathrm{F}_{\text {Time }}=7.685, \mathrm{R}^{2}$ Time $=0.307, p_{\text {Time }}=0.022, \mathrm{~B}$ in Figure 3.2). The interaction between treatment and time had no effect on foraging activity $\left(\mathrm{F}_{\text {Treatment }}=1.347, \mathrm{R}^{2}\right.$ Treatment $\left.=0.054, p_{\text {Treatment }}=0.220\right)$. In hives with Argentine ants, $26 \pm 4$ foragers (mean $\pm \mathrm{SE}$ ) returned per minute, compared to $16 \pm 3$ without ants (B in Figure 3.2). Overall, foraging activity peaked in March with $40 \pm 7$ (mean \pm SE) foragers returning every minute and was lowest in May (7 \pm 2 , B in Figure 3.2). 

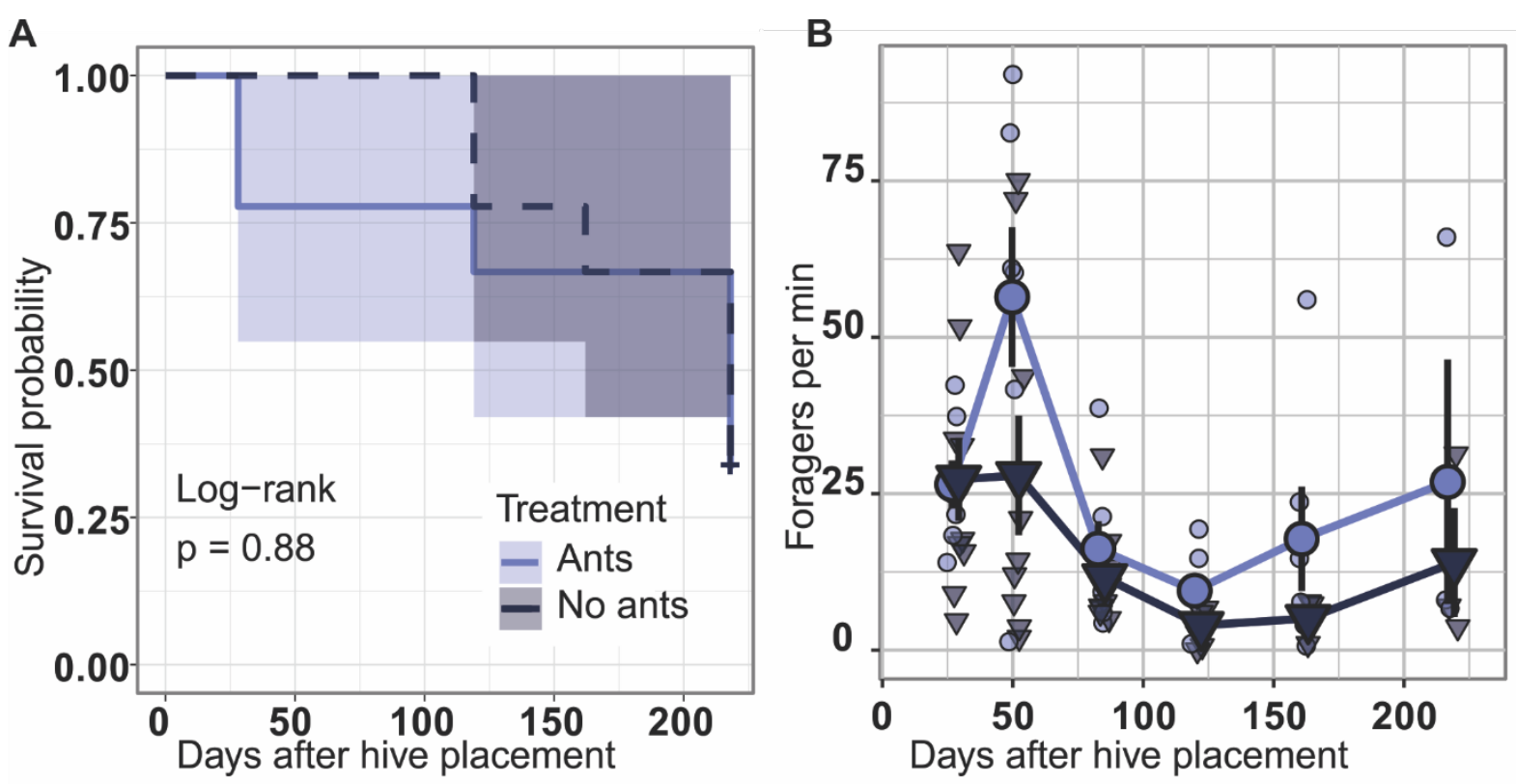

Figure 3.2: Survival and foraging activity of beehives with and without Argentine ants. Beehives were moved into sites in January (day 0) and observed until August (day 218). A: Kaplan-Meier curves showing survival probability with $95 \%$ confidence intervals in beehives from sites with (solid blue line) and without (dashed dark blue line) Argentine ants. The logrank test showed no difference between groups. B: Mean number of returning foragers per minute in beehives with (circles, blue colour) and without (triangles, dark blue colour) Argentine ants. Large symbols indicate the mean \pm one standard error, small transparent shapes show individual data points.

\subsubsection{Pathogen infections in beehives}

In order to determine if ant presence affects pathogen infections in beehives, we measured BQCV, DWV and KBV viral titres and N. ceranae and L. passim infection loads in beehives from January until August. The most commonly detected pathogen in honey bees was BQCV (94.85\% of samples across all time points), followed by DWV and L. passim (both $76.29 \%)$. The microsporidian N. ceranae was found in less than half of the samples $(45.36 \%)$ and KBV infections were rare and only detected in nine hives (9.28\%). All nine KBV-positive hives were also infected with DWV. Deformed wing virus titres were positive correlated with KBV titres ( $\mathrm{n}=9$ coinfected, Spearman's $\rho=0.867, p=0.002)$. The only other significant correlation in pathogen loads was found between BQCV and L. passim $(\mathrm{n}=70$ coinfected, Spearman's $\rho=0.356, p=0.003$, all other pathogen combinations $p>0.05$ ). Foraging activity was positively correlated with BQCV titres (Spearman's $\rho=0.303, p=0.009$ ) but not with other pathogens (all $p>0.05)$.

Out of the three viral pathogens tested, DWV showed the strongest seasonal variation (Figure 3.3). While the virus was detected in only two out of 18 hives in January, DWV prevalence increased from February onwards reaching 100\% in March but then dropped below 
$100 \%$ in April, May and June (A in Figure 3.3). In sites with Argentine ants, DWV in bees reached extremely high levels from autumn until early winter (mean viral load \pm SE: $16.756 \pm$ 12.236 in April, $1.243 \pm 0.317$ in May, $18.134 \pm 8.495$ in June) before decreasing in late winter (August: $0.003 \pm 0.003$, A in Figure 3.3). In hives without Argentine ants, DWV peaked in April after which infection levels slowly decreased (mean viral load \pm SE: $3.718 \pm 3.322$ in April, $2.602 \pm 2.476$ in May, $0.557 \pm 0.556$ in June, $<0.001$ in August, A in Figure 3.3). Viral titres of DWV differed significantly between treatments but also between sampling sites and time points, while no significant treatment-time interaction was found (PERMANOVA: $\mathrm{F}_{\text {Treatment }}=4.653, \mathrm{R}^{2}$ Treatment $=0.046, p_{\text {Treatment }}=0.026, \mathrm{~F}_{\text {Site }}=43.324, \mathrm{R}^{2}$ Site $=0.131, p_{\text {Site }}=0.007$, $\mathrm{F}_{\text {Time }}=2.324, \mathrm{R}^{2}$ Time $=0.114, p_{\text {Time }}=0.006, \mathrm{~F}_{\text {Interaction }}=1.837, \mathrm{R}_{\text {Interaction }}=0.090, p_{\text {Interaction }}=$ $0.074)$.

Black queen cell virus loads were highest in January and decreased throughout the season with the lowest levels in August (B in Figure 3.3). Black queen cell virus levels were significantly affected by Argentine ants and sampling time (PERMANOVA: $\mathrm{F}_{\text {Treatment }}=3.420$, $\mathrm{R}^{2}$ Treatment $=0.044, p_{\text {Treatment }}=0.004 ; \mathrm{F}_{\text {Time }}=0.578, \mathrm{R}^{2}$ Time $\left.=0.037, p_{\text {Time }}=0.046\right)$, but not by the treatment-time interaction or sampling site $\left(\mathrm{F}_{\text {Interaction }}=0.708, \mathrm{R}^{2}\right.$ Interaction $=0.045, p_{\text {Interaction }}=$ $0.652 ; \mathrm{F}_{\text {Site }}=1.382, \mathrm{R}^{2}$ Site $\left.=0.071, p_{\text {Site }}=0.184\right)$. Overall, BQCV levels were higher with Argentine ants, however, the differences in viral loads were much smaller than in DWV (mean viral load \pm SE with ants $0.016 \pm 0.010$ and without ants $0.001 \pm 0.0002$ ). The least prevalent out of the five pathogens tested in honey bees was KBV, which was found from April until June in two sites that had Argentine ant nests (C in Figure 3.3). 

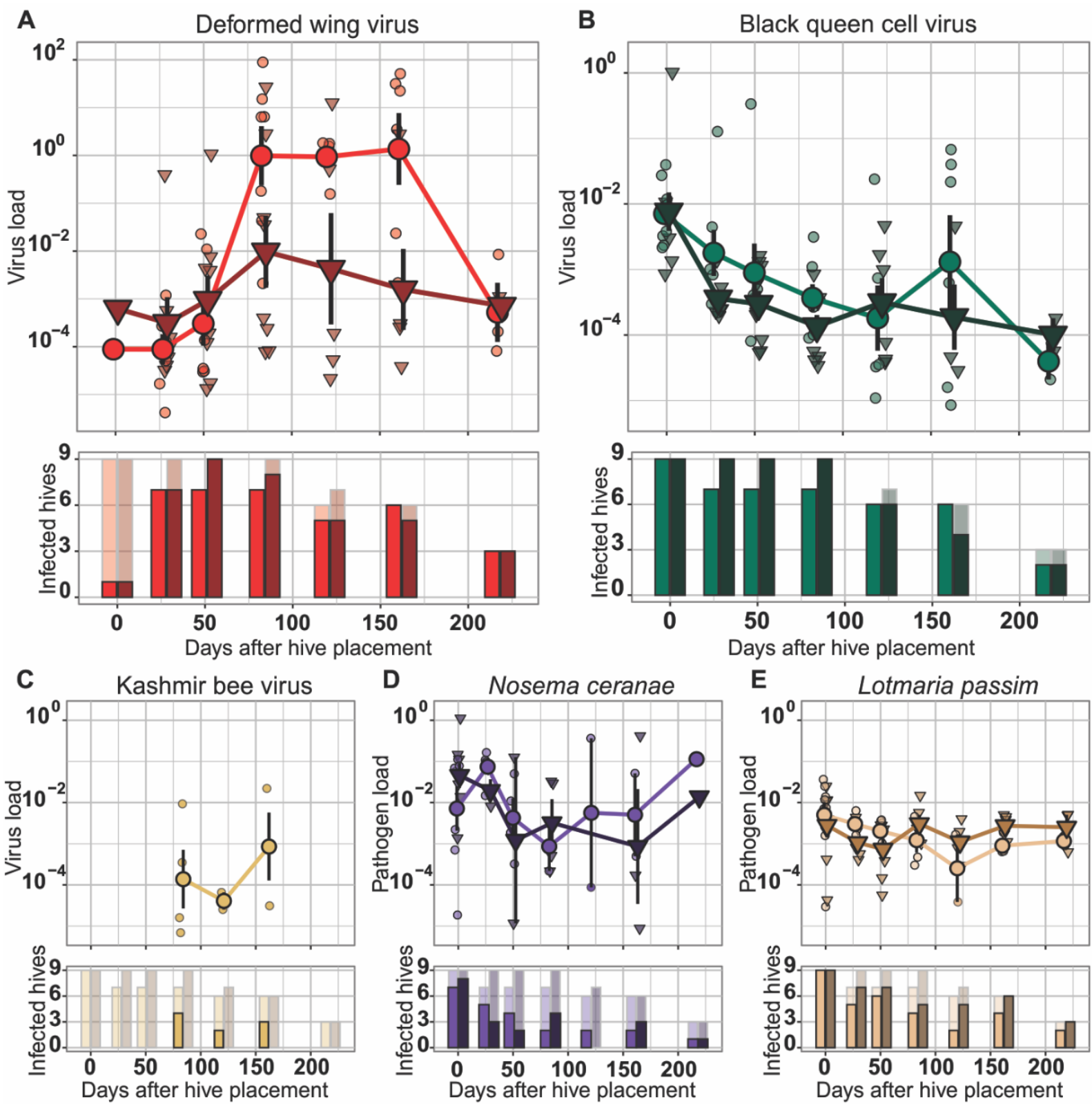

Figure 3.3: Pathogen load and prevalence in honey bee (A. mellifera) hives after beehives were placed in sites with and without Argentine ants (L. humile). Pathogens tested were deformed wing virus (A, red), black queen cell virus (B, green), Kashmir bee virus (C, yellow), $N$. ceranae (D, purple), and L. passim (E, beige). Beehives were moved into sites in January (day 0 ) and observed until August (day 218). Each of the five pathogens (A - E) has a top and bottom panel. Top: Virus or other pathogen loads in beehives from sites with Argentine ants (circles, light colour) and without Argentine ants (triangles, dark colour). Large symbols indicate the mean \pm standard error. Small symbols are individual data points. Note the different scale for deformed wing virus (A). Bottom: Pathogen prevalence: Bars indicate the number of beehives that tested positive for a pathogen in the treatment group with ants (left, light colour) and without ants (right, dark colour), transparent bars in the background indicate how many hives were alive in each group at the time point. 
The microsporidian $N$. ceranae was most prevalent in January ( $83.33 \%$ infected) but prevalence decreased during autumn and winter and was lowest in May when only two hives with Argentine ants were $N$. ceranae positive (D in Figure 3.3). Nosema ceranae infection levels were not affected by ant presence, samling site or time, or the treatment-time interaction

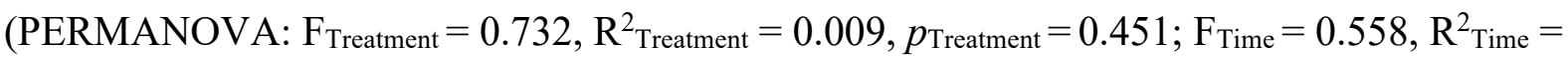
$0.036, p_{\text {Time }}=0.567 ; \mathrm{F}_{\text {Site }}=0.890, \mathrm{R}^{2}$ Site $=0.046, p_{\text {Site }}=0.489 ; \mathrm{F}_{\text {Interaction }}=1.492, \mathrm{R}^{2}$ Interaction $=$ $\left.0.096, p_{\text {Interaction }}=0.094\right)$. The trypanosomatid L. passim was most prevalent in January $(100 \%$ of hives infected) after which prevalence slightly decreased (E in Figure 3.3). Although higher at first, from April onwards, L. passim levels were lower in colonies with ants, this difference was not statistically significant (PERMANOVA: $\mathrm{F}_{\text {Treatment }}=1.697, \mathrm{R}^{2}$ Treatment $=0.018$, $\left.p_{\text {Treatment }}=0.217 ; \mathrm{F}_{\text {Interaction }}=2.120, \mathrm{R}_{\text {Interaction }}=0.111, p_{\text {Interaction }}=0.066\right)$. Sampling site and sampling time point significantly affected L. passim levels $\left(\mathrm{F}_{\text {Time }}=0.821, \mathrm{R}^{2}\right.$ Time $=0.043, p_{\text {Time }}$ $=0.005 ; \mathrm{F}_{\text {Site }}=4.136, \mathrm{R}^{2}$ Site $=0.172, p_{\text {Site }}=0.006$ ). Pathogen levels were highest in April and lowest in May (mean pathogen load \pm SE: $0.004 \pm 0.002$ and $0.001 \pm 0.0003$, respectively). The sites with with highest and lowest L. passim levels were both free of Argentine ants (HOW and HL2 in Figure S3.1; mean pathogen load \pm SE: $0.004 \pm 0.001$ and $0.001 \pm 0.0004$, respectively).

\subsubsection{Viral infections in ant nests}

The three bee-infecting viruses BQCV, DWV and KBV were found in all three ant colonies in January on the day the beehives were moved into the field sites (Figure 3.4). One site (WAI in Figure S3.1) had beehives before we moved the experimental colonies in and showed the highest DWV tires in January. Throughout the experiment, differences between ant colonies were large. The highest variation in viral loads was found for DWV with extremely high levels in May (A in Figure 3.4). Noticeably, KBV was found in all three Argentine ant nests throughout the study (except one nest in March, $\mathrm{C}$ in Figure 3.4) but was much rarer in honey bees. 

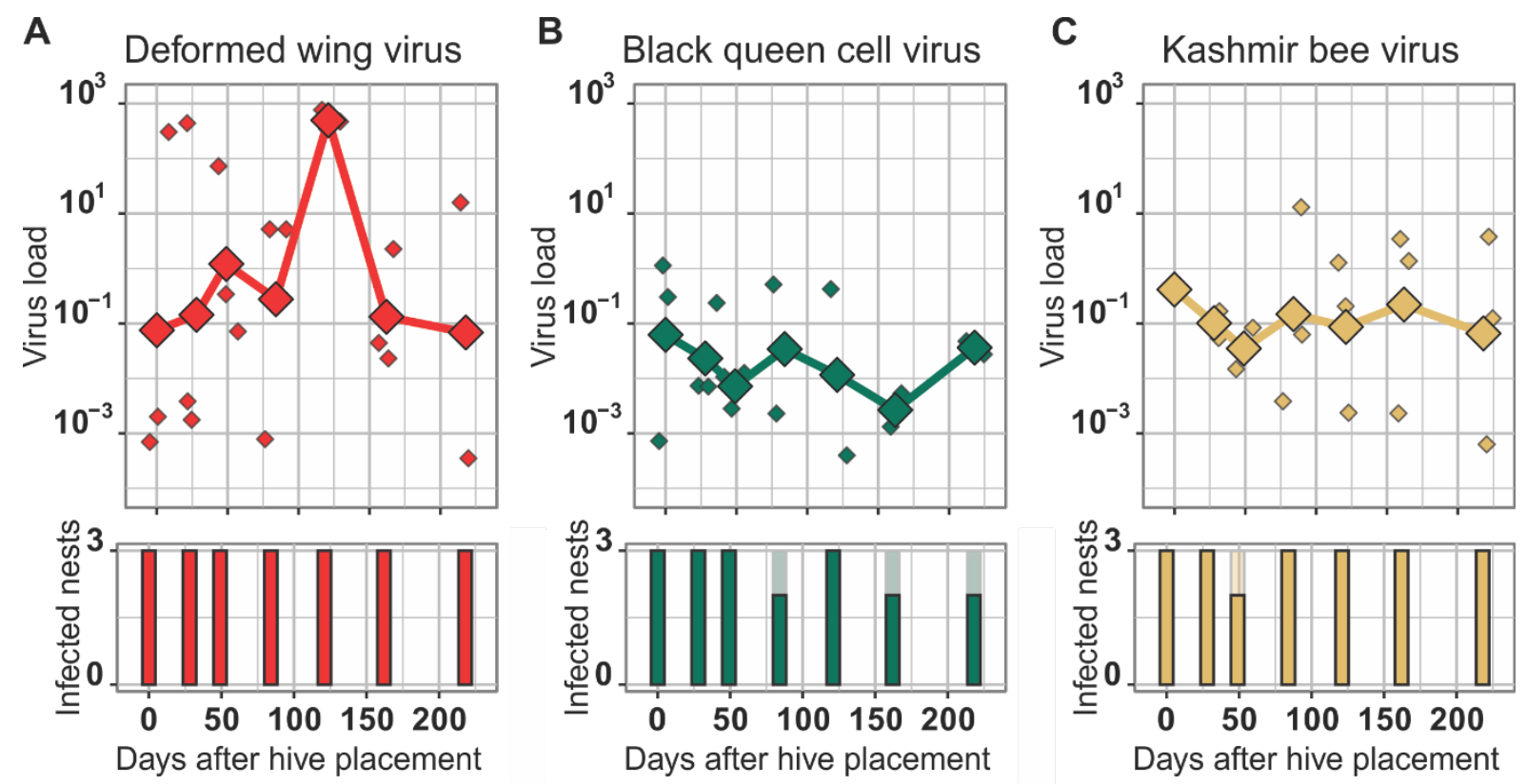

Figure 3.4: Viral load and prevalence of bee-associated viruses in Argentine ants (L. humile) after beehives were placed next to ant nests. Viruses tested were deformed wing virus (A, red), black queen cell virus (B, green), and Kashmir bee virus $(C$, yellow). Beehives were moved into sites in January (day 0) and observed until August (day 218). Top: Mean viral load over time is indicated by large filled diamonds, with individual data points shown by small diamonds. Bottom: bars indicate the number of nests that tested positive for the virus.

In March, we compared the ant nests in the experiment with four ant nests in the same region but outside of apiaries. Three out of four ant nests that were sampled outside apiaries were DWV positive, however, DWV load in those was at least three magnitudes lower than DWV load in ant nests with beehives (Kruskal-Wallis rank-sum test, $\chi^{2}=3.857, p=0.049$, Figure S3.2). Kashmir bee virus and BQCV viral levels were not significantly different between ant nests with and without beehives (both $p>0.05$ ), however, only one nest outside an apiary tested positive for BQCV (Figure S3.2).

We screened Argentine ants for an additional eleven viruses that have only been detected in Argentine ants and ABPV which had previously been found in Argentine ants (Gruber et al. 2017, Viljakainen et al. 2018). Five viruses, LhuBLV1, LhuEV1, LhuPLV1, LhuTLV1, and LhuPCV2, were always present in all three ant colonies. The largest variation in viral loads was found in LhuPCV2, while LhuBLV1, LhuEV1, LhuPLV1, and LhuTLV1 showed less variation (Figure S3.3). Linepithema humile C-virus 1 and LhuPiLV1 were found in most nests and present throughout the whole sampling period (Figure S3.3). LHUV-1 and LhuRLV1 were less prevalent and were absent in some months (Figure S3.3). Acute bee paralysis virus, LhuPCV1, and LhuQLV1 were not detected. The comparison between ants 
inside and outside of apiaries in March did not show differences between groups in viral loads of the nine ant-specific viruses we detected (Kruskal-Wallis rank-sum test all $p>0.05$ ).

Finally, we tested whether viral loads in beehives correlated with viral loads in ant colonies from the same apiary. Deformed wing virus load in bees was not correlated with DWV in ants (Spearman's $\rho=0.070, p=0.686$ ) but BQCV and KBV loads in bees were positively correlated with BQCV and KBV loads in ants (Spearman's $\rho=0.336, p=0.045$ and $\rho=0.339$, $p=0.043$, respectively).

\subsection{Discussion}

We showed that an ant species that is considered a pest in apiaries can affect seasonal viral infection dynamics in honey bees. We found that BQCV and DWV titres were significantly affected by ants, while the non-viral pathogens, N. ceranae and L. passim, were not affected by ant presence. The rarest virus detected, KBV, was found in ants throughout the study, yet only a few beehives showed KBV infections in sites with ants in late autumn. Deformed wing virus showed strong seasonal variation with a higher amplitude of increase in autumn when Argentine ants were present. Honey bee population dynamics can affect the life cycle of viruses and cause seasonal patterns in infection levels (Tentcheva et al. 2004, Runckel et al. 2011, Beaurepaire et al. 2020), yet ant presence seems to affect these patterns. Infection levels of BQCV and KBV in bees correlated with those found in ants from the same apiary suggesting similar seasonal patterns in both species or inter-species virus transmission.

We did not find evidence for increased hive mortality associated with Argentine ant presence throughout the experiment, nevertheless, shifts in viral infections and coinfection networks have been associated with colony collapse and reduced bee health (Cornman et al. 2012). Accumulating stressors and interactions between them, in particular between pathogens can accelerate honey bee mortality (Cornman et al. 2012, Goulson et al. 2015). The mechanisms by which ants could affect viral disease in bees are unknown. Here we discuss three possibilities: ants as biological vectors, ants as mechanical vectors, and stress-induced viral disease.

\subsubsection{Ants as biological vectors}

Argentine ants may be biological hosts for bee-infecting viruses, which is supported by our finding of BQCV, DWV and KBV in ant nests at the start of the experiments. Although it should be noted that one ant nest had beehives within foraging distance before the experiment began. It has been hypothesised that Argentine ants function as a pathogen reservoir from 
which viruses are spilled into honey bee populations (Sebastien et al. 2015). Active viral replication is a requirement for an organism to act as a biological vector for a virus (Yañez et al. 2020). Two bee-infecting RNA viruses (DWV and KBV) show evidence of active replication in Argentine ants (Gruber et al. 2017, Dobelmann et al. 2020). Other bee viruses were confirmed to replicate in other ants species, such as chronic bee paralysis virus in Camponotus vagus (Celle et al. 2008) and ABPV in Lasius species (Schläppi et al. 2020). Immune gene expression changes in Argentine ants correlate with BQCV, DWV and KBV infection levels, indicating an immune reaction that could be caused by replicating viruses (Lester et al. 2019). Ant-specific viruses, however, trigger a stronger immune reaction (Lester et al. 2019) and no evidence has been found for BQCV replication in Argentine ants (Gruber et al. 2017). Whether ants are an active host for these viruses and whether bees transmit viruses to ants is an ongoing area of research (Baty et al. 2020, Payne et al. 2020). For some beeinfecting viruses, viral levels and prevalence typically decrease outside apiaries (Gruber et al. 2017, Payne et al. 2020) suggesting that ants acquire viruses through scavenging on infected bees (Schläppi et al. 2019), which corresponds with our observations of ants consuming brood and dead bees and decreased DWV levels in ants outside apiaries. For KBV, on the other hand, it appears less likely that infections in ants depend on viral spillover from honey bees. We found no difference in KBV titres between Argentine ants in apiaries and other sites in the March sample. Others have made similar observations for KBV in Solenopsis invicta (Payne et al. 2020). The prevalence of KBV can reach higher levels in Argentine ants than in honey bees (Dobelmann et al. 2020). Studies on the Argentine ant virome have frequently detected KBV (Viljakainen et al. 2018, Felden et al. 2019) suggesting that, despite its name, the virus could possibly be closer associated with ants or other non-bee insects. None of the beehives in our experiment were found to be infected with KBV until April even though KBV-infected ants inside the apiary (and hives) could have exposed bees to the virus. The accumulated exposure of KBV through infected ants inside the beehive could have led to KBV infections in some beehives.

\subsubsection{Ants as mechanical vectors}

As mechanical vectors ants could passively acquire and mechanically transmit the virus, without supporting viral replication prior to transmission (Schläppi et al. 2019, Payne et al. 2020). A recent study showed that bees can vector microsporidian parasites without being actively infected (Graystock et al. 2015). After the host species deposits parasites on flowers, a different bee species that can not be affected by the microsporidian moves the pathogen to 
different flowers from where the host species then acquire infections (Graystock et al. 2015). The study shows that mechanical vectors and shared foraging environments provide a route for transmission (Graystock et al. 2015). We discuss a similar mechanism by which ants could mechanically vector bee viruses here. Even before the start of the experiment, ants may have been exposed to and acquired bee viruses from other virus-positive species (virus provider). Bee-infecting viruses such as BQCV, DWV, and $\mathrm{KBV}$, have been found in a vast number of pollinators and associated arthropods (Singh et al. 2010, Levitt et al. 2013, Dolezal et al. 2016, Alger et al. 2019, Dobelmann et al. 2020). When Argentine ants and honey bees interacted, we found elevated DWV levels in both species, therefore the interaction might promote DWV prevalence or infection levels. Ants could passively acquire DWV from entering beehives, consuming infected bees and bee products (Schläppi et al. 2019, Payne et al. 2020). Simultaneously, ants moving within and between beehives could then disperse the virus and may infect additional bees within the hive or transmit the virus to neighbouring hives. Yet, viral prevalence and titres vary temporally, making it difficult to infer the direction of transmission (Runckel et al. 2011). DWV titres positively correlated within sampling sites, which indicates transmission between hives within the same site or site effects that were not measured in this study. Bees entering neighbouring colonies (drifting) can transmit disease (Yañez et al. 2020), whether ants facilitate transmission between hives has not been studied yet.

\subsubsection{Stress-induced viral disease}

Even without transmitting viruses the activity of ants entering the hive to rob food stores or prey on bee brood causes additional stress to the bee colony (Payne et al. 2020). The accumulation of stressors is known to decrease bee health (Nazzi et al. 2012, Goulson et al. 2015). Examples show how stressors can affect bee disease. Stressful rearing conditions, for instance, increase susceptibility to L. passim (Schwarz et al. 2016). Neonicotinoids increase the susceptibility to $N$. ceranae (Wu et al. 2012) and promote DWV replication by disrupting antiviral responses controlled by the Toll pathway (Nazzi et al. 2012, Di Prisco et al. 2013). The parasitic Varroa mite can suppress the immune function in bees which is correlated with increased virus replication (Yang and Cox-Foster 2005, Nazzi et al. 2012, Di Prisco et al. 2016). Multiple stress factors may synergistically promote DWV replication (Nazzi and Pennacchio 2014). The elevated DWV levels in bees with Argentine ants suggest that ants could be such as stress factor that potentially affects immune responses. Deformed wing virus reduces the expression of a transcription factor involved in Toll-mediated antiviral immunity, which 
possibly allows the virus to persist at low rates (Nazzi et al. 2012). Nazzi et al. (2012) argue that stressors that activate responses that depend on this transcription factor overwhelm immune responses causing viral increases. Multiple stressors such as Varroa, poor nutrition, and insecticides interfere with this transcription factor, which could impact immunocompetence and trigger changes in DWV-mite interactions (Nazzi and Pennacchio 2018). Whether ant presence triggers stress responses or affects immune signalling pathways needs to be tested.

Deformed wing virus is one of the main viruses linked to hive collapses (Highfield et al. 2009, Dainat et al. 2012). Low-level covert DWV infections, such as we observed during summer and early autumn can have long-term effects on colony survival by impacting foraging activity (Benaets et al. 2017). Such long-term effects may then affect susceptibility to secondary pathogen infections (Seabloom et al. 2015, Di Prisco et al. 2016). We found that KBV only infected colonies in late autumn when DWV levels in hives exposed to Argentine ants were high. Perhaps KBV was acquired when colonies were weakened by DWV, Argentine ant presence or other pathogens and stressors. We found $N$. ceranae and L. passim to follow typical seasonal dynamics with high incidences in late summer and early autumn (Runckel et al. 2011, Vejnovic et al. 2018) regardless of ant presence. Positive correlations between the two pathogens are also in accordance with other studies (Tritschler et al. 2017).

Regardless of the mechanisms, increased viral loads and shifts in pathogen networks associated with ant presence can be detrimental to bees. In general, infections with one pathogen seem to positively correlate with other infections (Cox-Foster et al. 2007, Cornman et al. 2012). Although, competition for resources between viruses can also exclude secondary infections (Mordecai et al. 2016a). With the arrival of Varroa in New Zealand, viral pathogen networks in bees shifted (Mondet et al. 2014). Effects from Argentine ants on the bee pathogen communities may be more subtle, yet increases in DWV levels in autumn are worrying as they may result in bee health decline and increased overwinter mortality (Highfield et al. 2009, Cornman et al. 2012, Dainat et al. 2012). Only viral infections in bees were affected by ants and we did not observe changes in the non-viral pathogens that are specific to bees (N. ceranae and L. passim). These results may indicate that viral transmission could be the main mechanism for increased viral loads in bees or that if ants act as a stressor only some pathogens are affected. Novel vectors and ways of dispersing honey bee-associated viruses could possibly affect wider pollinator communities through viral spillover (Fürst et al. 2014, Manley et al. 2019). Insights 
into pathogen transmission and the role of ants within transmission networks will aid in decisions about controlling host species to manage disease (Dobson 2004). 


\section{Chapter 4:RNA sequencing reveals invasive ants are linked to increased Sacbrood virus levels and trigger transcriptomic responses in honey bees}

\subsection{Abstract}

Several RNA viruses that are associated with honey bee colony losses have been found in ant species. Yet, it is unknown whether the introduction of ants, which may host or vector viruses, affects viral networks. Here, we used RNA sequencing (RNA-seq) to study whether Argentine ants (Linepithema humile) affect the RNA virome and transcriptomic responses in European honey bees (Apis mellifera). We found evidence for 12 different RNA viruses in beehives which included three plant-infecting viruses and one unclassified virus that has not previously been detected in honey bees. Deformed wing virus was the most abundant virus and was associated with high Sacbrood virus and black queen cell virus titres in some hives. Sacbrood virus and the plant-associated Tomato ringspot virus had significantly higher titres in beehives with ants. However, as these viruses are not found in Argentine ants, it is unlikely that they were vectored by ants. Ant-presence may affect viral dynamics through other mechanisms. Honey bee genes that were differentially expressed with ant presence were associated with the cuticle and overlapped with transcriptomic responses typically associated with viruses and immune activation. We suggest that ants act as a stressor that, possibly in combination with other stressors, affects immune function and thereby facilitates viral infections that induce transcriptomic responses. Improved understanding of ant-bee interactions and identification of stress responses can help prevent honey bee disease. 


\subsection{Introduction}

The role of viral infections in honey bee colony losses has boosted interest in the field of bee virus research (Runckel et al. 2011, Cornman et al. 2012, McMenamin and Genersch 2015, Gisder and Genersch 2017, Grozinger and Flenniken 2019). Increasing evidence is pointing towards networks of interacting viruses that together affect the health of honey bees (Cox-Foster et al. 2007, McMahon et al. 2015). Virus-virus dynamics within the bee host can be complex and highlight the need to study viruses within their communities rather than viral species individually. Multiple viruses or viral strains interact and can compete for resources within bees thereby affecting viral dynamics (Chen et al. 2004, Mordecai et al. 2016a). Deformed wing virus (DWV), for instance, one of the main viruses associated with hive collapses (Highfield et al. 2009), can facilitate secondary infections by reducing honey bee immunity (Di Prisco et al. 2016). The spread of the parasitic Varroa mite that vectors DWV further affects virus dynamics (Martin et al. 2012). Deformed wing virus infections in bee populations free of Varroa often persist covertly with low levels of virus replication. However, Varroa-mediated DWV transmission has rapidly altered the virulence and evolution of DWV strains in bee populations (Wilfert et al. 2016, Natsopoulou et al. 2017, Gisder et al. 2018, Remnant et al. 2019). Varroa-presence and pathogen infections can activate specific transcriptomic responses in the honey bee host (Doublet et al. 2017). Particularly, the activation of immune pathways such as the RNA-interference signalling pathway is characteristic for viral infections (Brutscher et al. 2015, 2017).

Many viruses that infect honey bees are multi-host viruses (Singh et al. 2010, Levitt et al. 2013). It is not well understood whether the introduction of new host species or stressors has the potential to induce changes in viral networks (Faillace et al. 2017). Multi-host viruses can be disproportionally affected by the abundance of certain hosts (Kilpatrick et al. 2006). Several bee-infecting RNA viruses have been found in ants (Sebastien et al. 2015, Gruber et al. 2017, Dobelmann et al. 2020, Payne et al. 2020). The most commonly detected viruses in ant species have all been previously associated with honey bees (Baty et al. 2020). Although it remains to be seen whether ant-bee interactions and inter-species viral transmission cause this finding or whether this result is due to a testing bias given the limited knowledge of antassociated viruses (Baty et al. 2020). The number of known Argentine ant viruses has rapidly increased in recent years through RNA sequencing (RNA-seq) (Sebastien et al. 2015, Viljakainen et al. 2018, Baty et al. 2020). Transcriptomic responses in ants to these antassociated viruses seem strong in comparison to responses to bee-associated viruses (Lester et 
al. 2019), which could suggest a limited ability of bee-associated viruses to infect ants or of the ant host to respond to bee-associated viruses. Yet, the frequent detection of bee-infecting viruses in species such as Argentine ants, sometimes with high viral titres (Gruber et al. 2017), has led to the assumption that the species could act as a viral reservoir (Sebastien et al. 2015). Ants possibly disperse viruses as a 'mechanical vector' which potentially affects transmission to honey bees (Payne et al. 2020).

The vast majority of bee-infecting viruses are part of the Dicistroviruses or Picornaviruses families and have positive-sense single-stranded RNA (+ssRNA) genomes (Chen and Siede 2007, Bonning and Miller 2010, Grozinger and Flenniken 2019). The high mutation rates in RNA viruses (Domingo and Holland 1997, Drake and Holland 1999) can hinder detection through PCR-based methods but do not limit next-generation sequencing techniques that do not require prior knowledge of viral genome sequences, such as RNA-seq. A remarkable diversity of viruses in arthropods had been revealed through RNA-seq (Li et al. 2015, Shi et al. 2016). High-throughput sequencing has become a popular tool to study insect virus communities, as insects are important for ecosystem function, food production or as pathogen vectors. Full-length viral genomes can be assembled bioinformatically using RNAseq data, allowing for phylogenetic analyses and detection of recombination events (Liu et al. 2011). Such genomic methods have, for instance, led to the discovery of the Lake Sinai virus (LSV) group, a widespread virus associated with bee colonies (Runckel et al. 2011), and are constantly discovering new bee-associated viruses (Remnant et al. 2017, Galbraith et al. 2018).

In this study, we use RNA-seq to study whether Argentine ants affect viral dynamics in honey bees. Argentine ant presence is associated with reduced hive survival (Brenton-Rule 2018) and increased DWV infection levels (discussed in Chapter 3). This ant species was first observed in New Zealand in 1990 (Green 1990, Corin et al. 2007) and has since become a pest in households as well as apiaries (Stahlmann-Brown et al. 2020). To improve the understanding of ant-bee interactions and how these interactions affect bee disease and transcriptomic responses, we look into RNA virus infections in honey bees in the presence and absence of Argentine ants. We hypothesise that Argentine ants act as a stressor or virus vector and thereby increase viral infections that can trigger antiviral immune responses in honey bees. 


\subsection{Material and methods}

\subsubsection{Experimental design and RNA library preparation}

Honey bee samples were collected in the Northland region of New Zealand as part of an experiment in which we observed the effects of Argentine ants on beehives. The experiment is described and discussed in detail in Chapter 3. Briefly, beehives were placed in six different sites, three with and three without Argentine ants, in January 2019 and monitored over five months (Figure 4.1 and Figure S4.1). For this study, we used 11 samples from honey bee colonies collected in June 2019. Six samples were collected from three sites without Argentine ants (two each) and five from three sites with Argentine ants (two each except RAN in Figure S4.1). Bees were collected from inside the hive, frozen in a liquid nitrogen dry shipper while in the field and transferred to $-80^{\circ} \mathrm{C}$ until analyses. Pools of 25 bees per hive were used for molecular analyses, which statistically allowed us to detect a virus that infects at least $4 \%$ of the bee population. Samples were washed with ice-cold phosphate-buffered saline (PBS, Ambion, Texas, USA) and RNA was extracted using the Direct-zol RNA Miniprep Plus Kit (Zymo Research, California, USA) following the manufacturer's instructions. RNA quality was checked using an RNA 6000 Nanochip on the Agilent 2100 Bioanalyzer (Agilent Technologies Co. Limited, Belgium), according to the manufacturer's instructions and then preserved in RNAStable (Biomatrica Inc., California, USA) for shipment to BGI Genomics (Hong Kong) for Illumina $1.9 \mathrm{Hi}$-seq 150 base pair (bp) paired-end sequencing. Adaptor trimming, filtering of contamination and low-quality reads were performed by BGI Genomics.

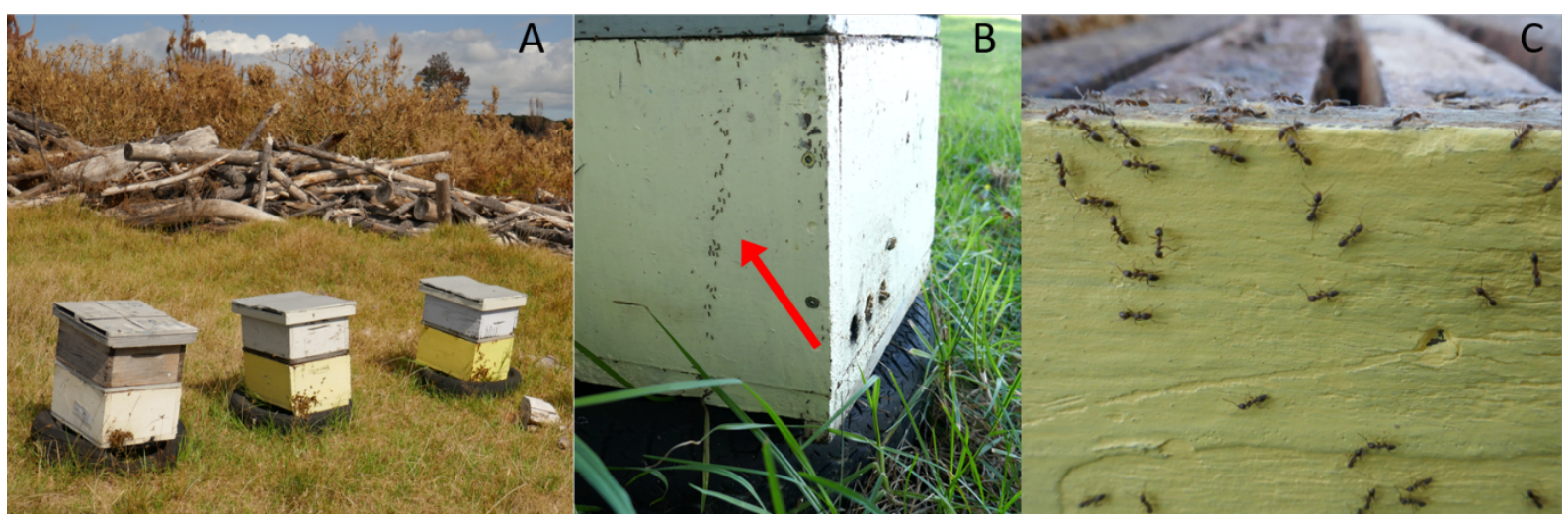

Figure 4.1: Photos of beehives with (and without) Argentine ants (L. humile). A: A sampling site with three small nucleus hives. Three hives were placed in each of the six different sites, half with Argentine ant incursions and the other half without this ant species. B: A trail of Argentine ants on the outside of a hive (red arrow). C: A close-up of Argentine ants on a beehive. Photos by Jana Dobelmann. 


\subsubsection{Quality control and ribosomal RNA filtering}

We checked read quality using fastqc v0.11.7 (https://www.bioinformatics. babraham.ac.uk/projects/fastqc/) and established that no further trimming or filtering was necessary. To determine whether ribosomal RNA (rRNA) removal had been successful we identified rRNA reads by de novo assembling reads from each sample using Trinity v2.9.1 (Grabherr et al. 2011) and using BLASTn searches (https://blast.ncbi.nlm.nih.gov/Blast.cgi, Altschul et al. 1990) against the honey bee reference transcriptome (Amel_HAv3.1). We then used rRNA matches as a reference and aligned reads from each hive to these references in HISAT2 v2.1.0 (Kim et al. 2019). Aligned reads were counted using Samtools v1.10 (Li et al. 2009). The percentage of rRNA was overall low, ranging from $0.20 \%$ to $0.67 \%$, indicating successful rRNA removal and was used to normalise read numbers.

\subsubsection{Honey bee transcriptome and differential gene expression}

To assess gene expression in honey bees, we followed the 'new tuxedo' protocol using HISAT2 v2.1.0 and StringTie v1.3.5 (Pertea et al. 2015). Clean paired-end reads were mapped to the honey bee reference genome (Amel_HAv3.1) in HISAT2 with default parameters using the annotation of the genome to produce sample-specific SAM files. Overall alignment rates ranged from $84.44 \%$ to $96.28 \%$ (Table S4.1). Samtools v1.10 (www.htslib.org) was used to convert SAM into BAM files and sort BAM files. Alignments were assembled in StringTie using the 'Transcript merge mode' (-merge) to merge assembled transcripts and create a uniform transcript assembly for all samples. Using this merged transcript assembly, transcript abundances were re-estimated where necessary and transcript abundance tables were created using the $-e$ and $-B$ parameters in StringTie. Transcripts were then matched to the reference transcriptome using gffcompare v0.11.2 (www.github.com/gpertea/gffcompare/ releases) and annotations were retrieved.

To analyse differential gene expression between beehives with and without ants we used DESeq2 (Love et al. 2014) in R v4.0.1 (R Core Team 2014). We generated a transcript count matrix at the gene level compatible with DESeq2 input using the prepDE.py script (https:/github.com/gpertea/stringtie/blob/master/prepDE.py), which uses coverage values from the StringTie $-e$ output. We filtered out genes with mean counts below ten, leaving 15,659 read counts and used the DESeq function with default parameters. $P$-values were adjusted using the false discovery rate method (Benjamini and Hochberg 1995) with $\alpha=0.05$. For visualisation purposes, the top 50 differentially expressed genes (DEGs) with the lowest 
adjusted $p$-values and an absolute fold change $(\mathrm{FC})>2$ were plotted in a heat map. For this plot, count estimates were log-transformed after adding a pseudo count of 1 and plotted using the pheatmap package (Kolde 2019). A principal component analysis (PCA) was performed using the plotPCA function integrated with DESeq2.

\subsubsection{Virus discovery in beehives}

Viral pathogens were detected and analysed in a separate pipeline using the reads that did not map concordantly onto the honey bee reference in the HISAT2 mapping. These unmapped reads were expected to include divergent honey bee sequences but also associated microbiota such as RNA viruses. Unmapped reads were de novo assembled using Trinity v2.9.1 (Grabherr et al. 2011) using default settings. The N50 (weighted median length of contigs) was 774 nucleotides. To identify putative viruses, contigs were compared against the non-redundant viral protein sequence database on GenBank (viral.nonredundant_protein.1.protein.faa, accessed 04/08/2020) using the ncbi_blast programme v2.7.1 and BLASTx searches with an $E$-value cut-off of $10^{-5}$. To identify and annotate high-confidence viral sequences, only matches with $>85 \%$ identity and $>300 \mathrm{bp}$ in length were included resulting in 12 viral species. In search of Argentine ant-associated viruses that may not be included in the NCBI viral protein database, we built a custom database that included Linepithema humile virus 1 (MF417634) discovered by Sebastien et al. (2015) and 12 viruses described in Viljakainen et al. (2018) (MH213235 MH213246, and LHUV1: MH213249). BLASTn searches were performed in Geneious v2019.0.4 with an $E$-value cut-off of $10^{-5}$, and matches with $<85 \%$ identity or $<300$ bp in length were filtered out. To visualise viral infection loads, Trimmed mean of $M$-values normalised counts were matched to the contigs with high-confidence viral hits.

\subsubsection{Viral infections in beehives with and without Argentine ants}

Differential expression analysis of virus contigs in hives with and without Argentine ants was performed in DESeq2 (Love et al. 2014). Estimated gene counts were generated with the abundance_estimates_to_matrix.pl script that is part of the Trinity pipeline (www.github.com/trinityrnaseq/trinityrnaseq/blob/master/util/abundance_estimates_to_matri x.pl). Contigs with mean counts below ten were filtered out leaving 8,819 read counts that were used in the analysis. $P$-values were adjusted using the false discovery rate method (Benjamini and Hochberg 1995) with a significance level of $\alpha=0.05$. To account for the size of the initial sequencing library, which included the mapped reads and manually calculated size factors that DESeq2 uses for read count normalisation. Size factors were calculated by taking the sum of 
mapped and unmapped reads within each library (after filtering out lowly expressed genes) divided by the median number of total reads. As a default, DESeq2 detects outliers by Cook's distance and does not provide $p$-values for outlier genes. We disabled this function as viral titres behave differently from gene expression and outliers may be common. DESeq2 results were mapped to the high-confidence viral hits to identify differential viral titres between hives with and without Argentine ants. Viruses that were found in less than 50\% of hives were excluded. Correlation of read count estimates between viruses was assessed using Pearson's correlation test. The PCA plots were generated using the plotPCA function integrated in DESeq2.

\subsubsection{Phylogenetic analysis}

One of the viruses discovered through BLASTx searches was Hubei picorna-like virus 15 (HPLV-15, YP_009336541.1), which had, to our knowledge, not been identified in honey bees before. Among the ten matches with the lowest $E$-value in this search were two unclassified Riboviria, HPLV-15 (APG77986) and Gorebridge virus (QAY29254), five unclassified viruses in the Picornavirales order, Bombus-associated virus Pic1 (QAY29255) and four Picornavirales spp. (QJI53523, -549 -581, and QKN88946), and as the only known bee-infecting virus, Aphid lethal paralysis virus (ALPV) in the Dicistroviridae family (ATJ04557 and QAY29255). We used phylogenetic analysis to identify the sequence homology of this unclassified virus to known bee pathogens. Therefore, contigs matching HPLV-15 and ALPV were compared with global virus isolates retrieved from GenBank (www.ncbi.nlm.nih.gov/genbank/). The analysis was performed in Geneious 2019.0.4 (Kearse et al. 2012) using the MrBayes plugin v3.2.6 (Huelsenbeck and Ronquist 2001). The two contigs matching ALPV and HPLV-15 were aligned with nucleotide sequences from GenBank using the MUSCLE algorithm and trimmed where necessary. The final alignment was 1,849 nucleotides covering the structural virus protein. Cricket paralysis virus (Cripavirus, NC_003924) and black queen cell virus (Triatovirus, NC_003784) were used as outgroups. Bayesian analyses were performed with 2,000,000 Markov chain Monte Carlo iterations with sampling every 500 iterations and a $10 \%$ burn-in period. Convergence was confirmed with high effective sample sizes $(>1000)$ for all parameters. 


\subsection{Results}

\subsubsection{Virus discovery in beehives}

We detected 12 contigs with high-quality viral matches in our bee samples (Table S4.2). Six to ten different viruses were discovered in each hive (Figure 4.2). Black queen cell virus (BQCV), DWV, Moku virus (MKV), Sacbrood virus (SBV) and Tomato ringspot virus (ToRSV) were found in every hive tested (Figure 4.2). Even beehives within the same field sites were infected with different viruses and every hive had its unique combination of viruses (except hive 9 and 16). Eight viruses found are known to infect bees, which included ALPV, BQCV, DWV, Kashmir bee virus (KBV), two LSV strains (South Africa-2 and Tonga), MKV and SBV. We also detected three viruses that are more typically associated with plants: Arabis mosaic virus (ArMV), ToRSV and White clover cryptic virus 2 (WCCV2). One contig matched an unclassified Riboviria Hubei picorna-like virus 15 (HPLV15, details Table S4.2). All identified viruses are +ssRNA viruses, except WCCV2 which is a double-stranded RNA (dsRNA) virus in the Betapartitivurs genus. No Argentine ant-associated viruses were found.

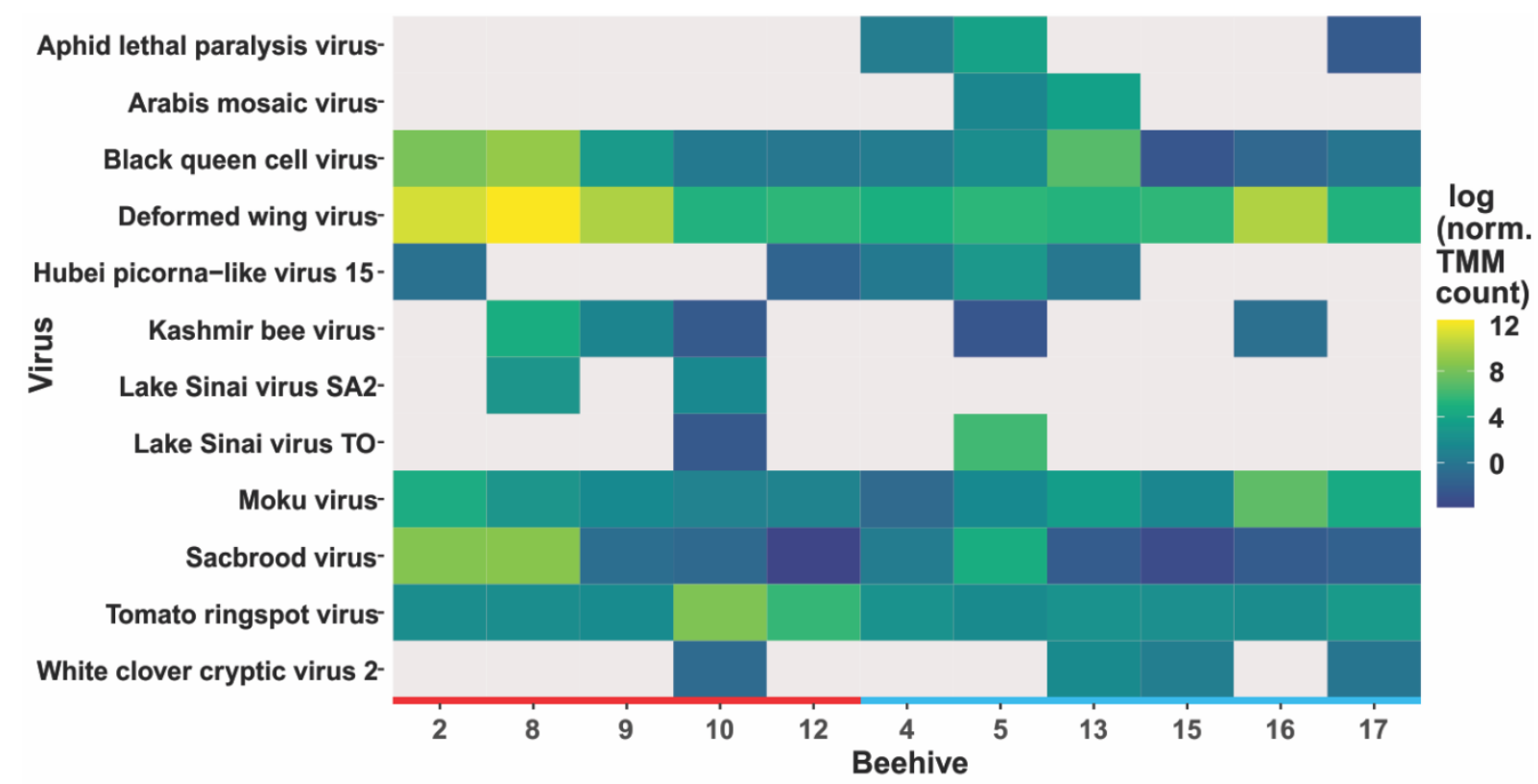

Figure 4.2: A heat map showing read numbers of viruses found in RNA sequencing libraries from beehives with and without Argentine ants. Beehives were either in sites with (red) or without Argentine ants (blue). Trinity de novo assembled transcripts were searched against the NCBI virus database using BLASTx with an $E$-value of $10^{-5}$, keeping matches $>300$ nucleotides and $>85 \%$ identity. Trimmed mean of $M$-values (TMM) normalised read count estimates indicate viral titres: blue showing low and yellow high viral levels, grey indicating virus absence. GenBank accessions: Aphid lethal paralysis virus (NP_733846), Arabis mosaic virus (YP_053924), black queen cell virus (NP_620564), deformed wing virus (strain A, NP_853560), Kashmir bee virus (NP_851403), lake Sinai virus SA2 (YP_009388492), lake Sinai virus To (YP_009388501), Moku virus (YP_009305421), sacbrood virus (NP_049374), tomato ringspot virus (NP_620765) and white clover cryptic virus 2 (YP_007889822). 
Deformed wing virus was the most abundant virus in seven out of the 11 beehives, exceptions were hive 13 in which BQCV had the highest titres, hive 5 in which LSV-To was the most abundant, and hive 10 and 12 in which, surprisingly, the plant virus ToRSV was the most abundant RNA virus (Figure 4.2). From the observations in Chapter 3, we know that only six hives sampled in June survived until they were monitored again two months later in August. Hive 8, in which we found the highest DWV levels survived while three other hives with relatively high DWV titres (2, 9 and 16) did not survive. Levels of BQCV, DWV and SBV were positively correlated (Pearson's correlation: BQCV and DWV: $\mathrm{r}=0.993, p<0.01, \mathrm{BQCV}$ and SBV: $\mathrm{r}=0.943, p<0.001$, DWV and SBV: $\mathrm{r}=0.923, p<0.001)$ while all other virus combinations were not correlated (all $p>0.05$ ).

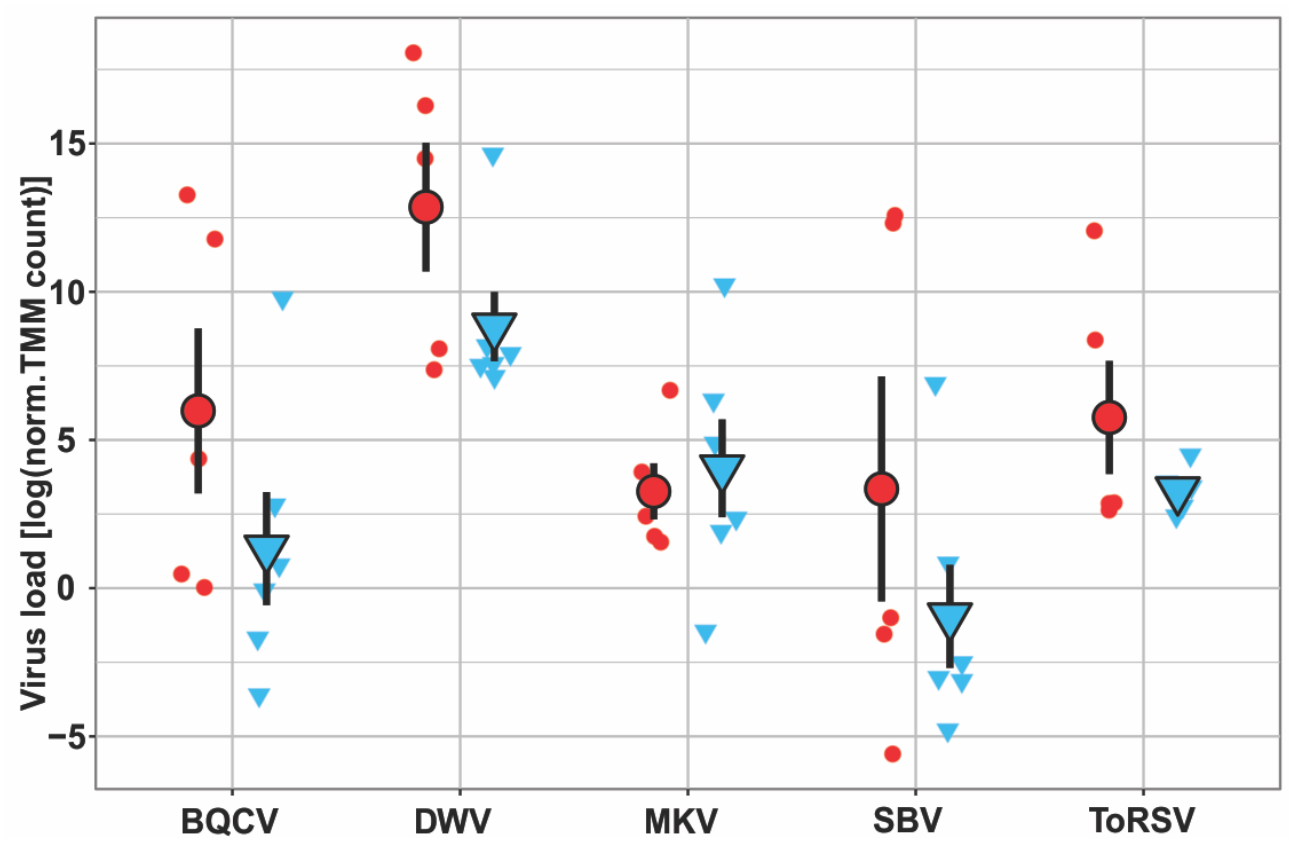

Figure 4.3: Virus titres in honey bees (A. mellifera) from sites with and without Argentine ants (L. humile). Virus titres are shown as trimmed mean of $M$-values (TMM) normalised read count estimates. Large symbols show the mean \pm one standard error and small symbols indicate individual values. Red circles show bees with and blue triangles bees without ants. Virus abbreviations: Black queen cell virus (BQCV), deformed wing virus (DWV), Moku virus (MKV), sacbrood virus (SBV), tomato ringspot virus (ToRSV). 


\subsubsection{Effect of Argentine ants on viral pathogens in honey bees}

We found 345 upregulated and 295 downregulated transcripts from the trinity de novo assembled reads that did not map Apis mellifera. Only five transcripts with high-quality virus matches were present in over $50 \%$ of hives and were mapped to the DEG analysis. Sacbrood virus levels were significantly higher in bees with Argentine ants $(\mathrm{FC}=6.634, p=0.050)$ and DWV was marginally increased with ant presence $(\mathrm{FC}=4.657, p=0.066)$. The plantassociated ToRSV had significantly higher titres in beehives with ants $(\mathrm{FC}=8.146, p<0.001)$. Black queen cell virus and MKV were not significantly affected by ant presence $(\mathrm{FC}=4.044$, $p=0.237$ and $\mathrm{FC}=-2.933, p=0.182$, respectively).

\subsubsection{Phylogeny of ALPV and an unclassified Riboviria}

The contig that matched the unclassified virus HPLV15 (YP_009336541.1, 86.4\% identity) was found in hives $2,4,5,12$, and 13. A manual BLASTx search revealed that the contig had high similarity with a virus that was only recently described, Gorebridge virus (QAY29254, 96.4\% identity). The phylogenetic analysis showed that the virus clustered closely with Gorebridge virus (Figure 4.4, MH614301). Gorebridge virus is a picorna-calici virus that was isolated from a Bombus spp. in Scotland (Pascall et al. 2019). Closely related to this virus is ALPV, a honey bee-infecting virus, which we also isolated from our samples and clustered with ALPV from a honey bee in Spain (JX045858 in Figure 4.4). Aphid lethal paralysis virus and the contig that matched HPLV15 and clustered with Gorebridge virus were different genes within the same Trinity read cluster. Trinity groups transcripts into clusters based on shared sequence content, which indicates high genetic similarity between the two viruses. 


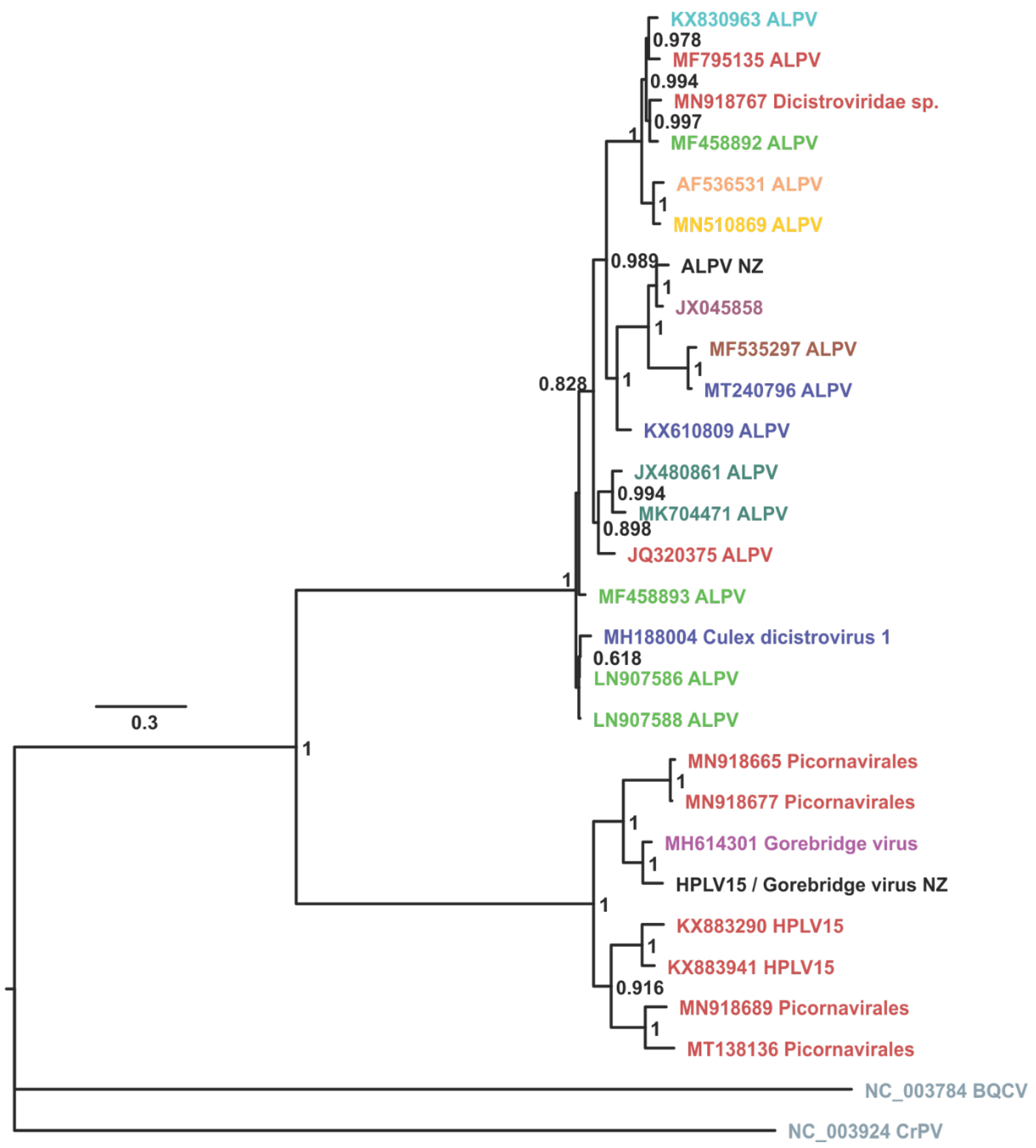

Figure 4.4: Maximum clade credibility tree for 1,931 nucleotides of the putative structural protein of Aphid lethal paralysis virus (ALPV) and the unclassified Hubei picorna-like virus 15 (HPLV15). Cricket paralysis virus (CrPV) and black queen cell virus (BQCV) were used as outgroups (grey). Posterior support $>0.7$ is given and the scale bar indicates the nucleotide substitutions per site. ALPV NZ and HPLV15/Gorebridge virus NZ were found in this study through RNA sequencing. The accession number and species name (if known) are shown in the branch label, which is coloured according to the geographic origin: Timor-Leste (light blue), China (red), Kenya (green), the Netherlands (orange), Zimbabwe (yellow), New Zealand (black), Spain (purple), Hungary (brown), USA (dark blue), Israel and Turkey (dark green) and the United Kingdom (pink). 


\subsubsection{Differential gene expression in beehives with and without Argentine ants}

Out of the 15,659 genes, 141 were identified as differentially expressed ( $p<0.05,|\mathrm{FC}|$ $>2$ ). Of those 69 were upregulated and 72 were downregulated in bees with Argentine ants (Table S4.3). The top 50 differentially expressed genes (DEGs) are shown in Figure 4.5. No distinct clustering by treatment group was observed in the PCA (Figure S4.2). Annotations for some genes could not be retrieved (MSTRG in gene name, Figure 4.5 and Table S4.3). Reasons for missing annotations could include novel transcripts without overlap in the reference, or transcripts being associated with multiple gene names when StringTie falsely joins overlapping genes.

Differentially expressed genes included genes involved in lipid catabolic processes such as the pancreatic triacylglycerol lipase-like gene (LOC411609) and multiple DEGs that are associated with the cuticle or pigmentation, including the pupal cuticle protein (LOC552685), cuticular protein 27 (CPR27), apidermin proteins (Apd-2 and Apd-3), and Yellow-y (Y-y). Other DEGs were associated with communication, including three Odorant binding proteins (Obp13, Obp16 and Obp18). Thirty-nine out of the 141 DEGs were uncharacterised whose functions are unknown and 23 had missing annotations. 


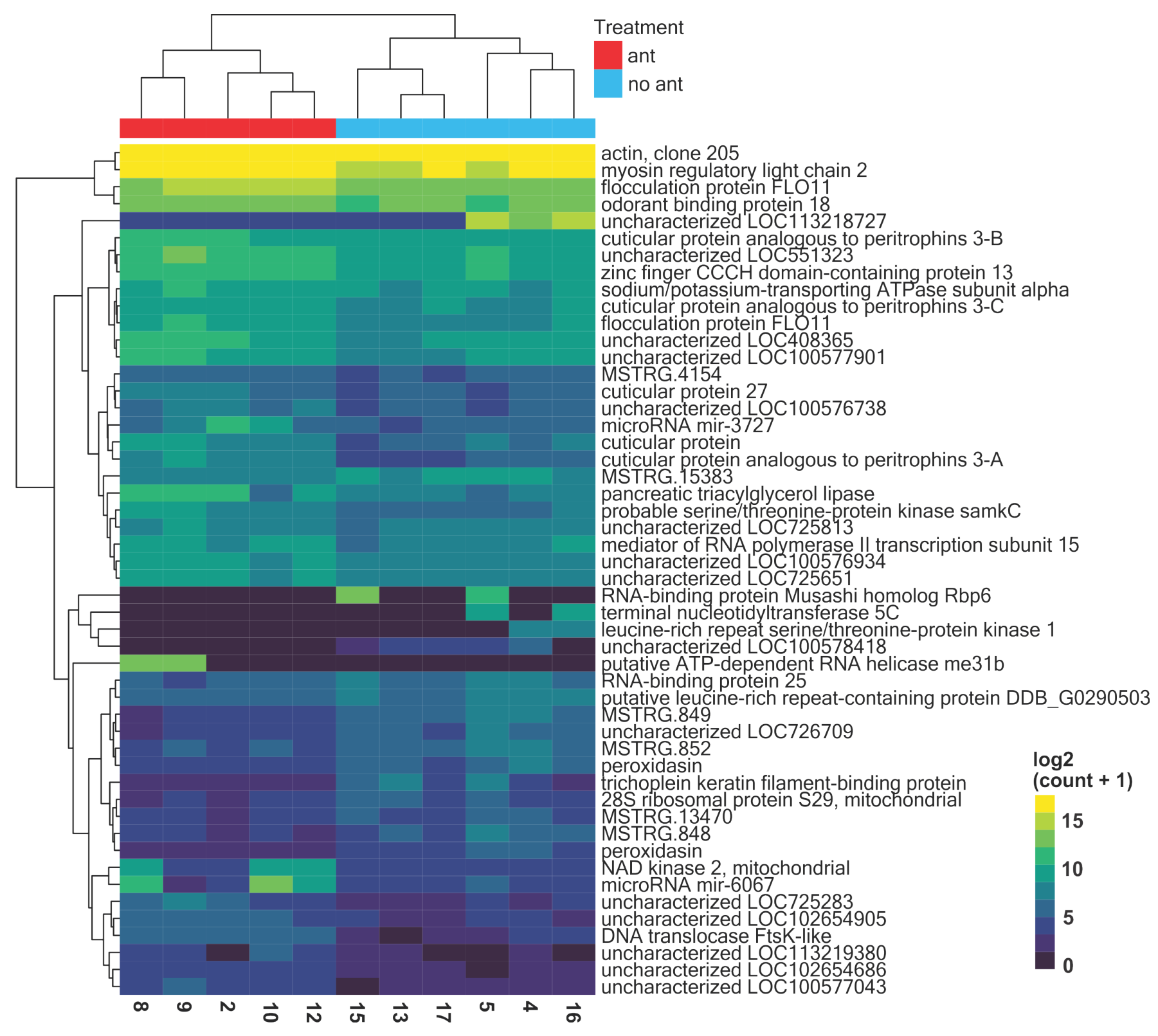

Figure 4.5: A heat map of the top 50 differentially expressed genes (DEGs) in honey bees with and without Argentine ants. Out of the 141 DEGs, 50 genes with the lowest adjusted p-value with an absolute fold change $>2$ were selected. Gene expression is shown as $\log 2$ transformed read counts after adding a pseudo count of 1 . Yellow indicates a high level of gene expression and dark blue a low expression level. Some transcripts do not have gene IDs associated and default StringTie IDs starting with "MSTRG" are shown. 


\subsection{Discussion}

In this study, we explored RNA virus infections in honey bee colonies that were infested with invasive Argentine ants using RNA-seq. As Argentine ants closely interact with bees and frequently carry bee-associated viruses, we hypothesised that ants would affect viral infections in bees. We found a significant increase of the bee pathogen SBV in bees sampled from sites with ants and observed that high DWV titres in some hives were associated with increases in BQCV and SBV levels. Additionally, three plant viruses and a novel virus that had not been found in honey bees before were detected. We observed several DEGs associated with Argentine ant presence and discuss below which biological functions they relate to and how ants could affect pathogen infections.

\subsubsection{Virus discovery and the association between ants and viral titres}

Argentine ant presence was associated with increased titres of SBV and ToRSV. Sacbrood virus levels were significantly increased in hives in sites that had ants, although we did not observe irregularities in brood patterns or diseased larvae that would be indicative of heavy SBV infections. Honey bee workers can be infected with SBV without showing any obvious symptoms (Chen and Siede 2007). Clinical symptoms for bee pathogens are often obscure or only observed at high infection levels (Chen and Siede 2007, Evans and Schwarz 2011, Gisder and Genersch 2017). Sacbrood virus is a common pathogen in honey bee colonies with high prevalence in spring and summer and typically lower prevalence in autumn (Tentcheva et al. 2004), which is when we sampled bees. Increased SBV levels detected in bees with ants may be driven by two hives ( 2 and 8 ) with viral levels many orders of magnitudes higher than in most other hives. Apart from SBV, these two hives had high BQCV and DWV titres, with DWV being the most abundant virus in both. Deformed wing virus, which in contrast to SBV, has been found in Argentine ants (Sebastien et al. 2015, Gruber et al. 2017, Dobelmann et al. 2020), was only marginally increased by their presence. Hight DWV levels may increase levels of secondary viral infections by disrupting immune signalling in bees (Di Prisco et al. 2016), which could have caused elevated BQCV and SBV titres associated with this virus.

Among the viruses we found in beehives were three that have been discovered in recent years, mostly through next-generation sequencing (Runckel et al. 2011, Mordecai et al. 2016b). These were LSV, ALPV and MKV. Two LSV strains were each found in two hives and LSV-To was the virus with the highest viral levels in one hive. Although LSV can reach high abundances in beehives (Runckel et al. 2011), codon usage suggests that, as opposed to other bee viruses, 
LSV may not have co-evolved with honey bees (Cornman 2019) and its effects on bee health are not well understood. Both strains we detected belong to the LSV-2 group (Remnant et al. 2017) and there is evidence for a negative correlation between LSV-2 levels and colony strength (Faurot-Daniels et al. 2020). Aphid lethal paralysis virus was first discovered in aphids (Hatfill et al. 1990) but has since been detected in honey bees in the USA (Runckel et al. 2011) and Europe (Granberg et al. 2013, Ravoet et al. 2013). We found ALPV in three hives. Phylogenetic studies suggest that ALPV consists of two distinct species, one of which can be found in honey bees (Liu et al. 2014). The strain in our study clustered with a Spanish ALPV strain from honey bees (JX045858). Moku virus is the most recently discovered out of the three viruses (Mordecai et al. 2016b) and we detected this virus in every hive. It is unknown whether MKV is pathogenic to bees. Replication of MKV has been found in Argentine ants and three wasp species (Vespula germanica, V. vulgaris and Polistes chinensis) but so far not in bees (Dobelmann et al. 2020).

While RNA sequencing is a powerful tool for virus discovery there are limitations. For instance, novel viruses that lack homology with known viruses cannot easily be identified and high viral titres are needed to acquire full-length genomes (Liu et al. 2011). For HPLV15/Gorebridge virus, we only acquired a 1,849 nucleotide-long contig that covered parts of the structural protein. The phylogenetic analysis suggested that the virus is closely related to Gorebridge virus (MH614301) which is a +ssRNA virus first described by Pascall et al. (2019). In their study, Gorebridge virus was extracted from bumble bees in Scotland and small RNA sequencing was used to test for virus replication but without showing active siRNA-based immune responses (Pascall et al. 2019). Many of the sequences that clustered with Gorebridge virus are unclassified Picornavirales collected from bird faeces suggesting the birds consumed infected insects. The closest relative to Gorebridge virus that is directly associated with honey bees is ALPV. Its detection in five hives from four different sites suggests that HPLV15/Gorebridge virus is relatively common in the sampled region.

\subsubsection{Bees could potentially vector plant viruses}

We found three viruses in beehives that are associated with plants: ArMV, ToRSV and WCCV2. These viruses presumably do not affect bee health but highlight the role pollinators may play in plant virus transmission (Shipp et al. 2008, Darzi et al. 2018). Tomato ringspot virus and ArMV are part of the Secoviridae family and infect crops such as tobacco, raspberries, peaches and grapes. Secoviridae are closely related to Dicistroviridae and Iflaviridae, the two main families of bee-infecting viruses (Grozinger and Flenniken 2019). Although Secoviruses infect plants, they can be found in honey bees (Granberg et al. 2013, Galbraith et al. 2018, 
Roberts et al. 2018) and other bee species (Schoonvaere et al. 2016). A seco-like virus was discovered in honey bees with a wide distribution over multiple continents suggesting a close, although potentially passive, association with bees (Galbraith et al. 2018). One study found active replication of a Secovirus in honey bees (Li et al. 2014), yet the results are debated and additional experiments are needed to support the findings (Miller et al. 2014). White clover cryptic virus 2 is the only dsRNA virus we found in this study and has only recently been recorded in New Zealand (Guy and Gerard 2018). The virus has rarely been found in insects, although WCC2 has been detected in bumble bees (Pascall et al. 2019). How this virus affects pasture plants such as clover and whether bees distribute the virus has not been studied. The fact that bees frequently carry plant viruses suggests they may play a significant role in vectoring plant viruses (Shipp et al. 2008, Darzi et al. 2018).

\subsubsection{Transcriptomic changes associated with Argentine ant presence}

Stress-related genes

We hypothesised that ant presence would affect viral infections in honey bees and that combined stress from ants and pathogen infections would result in transcriptomic responses. We found DEGs associated with ant presence that were related to immune responses previously found in studies on biotic stressors such as viruses or mite infestations. For instance, ant presence upregulated the pancreatic triacylglycerol lipase-like gene (LOC411609), which has been shown to increase in response to dsRNA and infections with DWV or SBV (Ryabov et al. 2016, Brutscher et al. 2017). This increase may be caused by higher SBV and slightly higher DWV titres in the ant group. Nosema infections, which we detected in bee samples in Chapter 3 can also affect lipid metabolism (Holt et al. 2013).

\section{Cuticle and exoskeleton-related genes}

We detected increased expression of several cuticle genes that may represent activation of repair mechanisms in response to parasites damaging the cuticle (Barribeau et al. 2014, Doublet et al. 2017). The pupal cuticle protein (LOC552685), for example, is part of a group of cuticular proteins with increased expression following immune challenge with a range of pathogens (Doublet et al. 2017) and ant presence. We also found a cuticular protein 27 (CPR27) expression increase, a gene that is differentially expressed over multiple time points in virusinfected bees (Chen et al. 2014, Brutscher et al. 2017). Increases in cuticular apidermin proteins (Apd-2 and Apd-3) that are otherwise associated with Nosema infections (Doublet et al. 2017) were also differentially expressed with ant presence. The gene Yellow-y $(Y-y)$, which controls the pigmentation pattern in the cuticle (Wittkopp et al. 2002) increased in bees with ants, while 
others found $Y-y$ decreases in association with viral infections (Chen et al. 2014, Galbraith et al. 2015, Ryabov et al. 2016).

\section{Communication-related genes and others}

Other DEGs were associated with communication, including three Odorant binding proteins (Obp13, Obp16 and Obp18). These proteins were found differentially expressed in dsRNA-treated bees (Brutscher et al. 2017) and are involved in the recognition and discrimination of odours, which are key for communication in social insects (Forêt and Maleszka 2006). Differential expression in odorant receptors is also found in Varroa-infested bees (Zanni et al. 2017). While some DEGs are uncharacterised (LOC102654905, LOC100576161, LOC724830, LOC552685), differential expression of these genes is consistent with other studies examining honey bee immune responses (Chen et al. 2014, Galbraith et al. 2015, Ryabov et al. 2016, Brutscher et al. 2017, Doublet et al. 2017), suggesting functions in immunity that are yet to be discovered. Transcriptomic studies are useful to identify stressor-specific markers, which can be used to monitor bee health (Dainat et al. 2012, Doublet et al. 2017, Zanni et al. 2017, Grozinger and Zayed 2020).

\subsubsection{Implications for understanding ant-bee interactions}

Although viral infections in honey bees have been extensively studied, gaps in our understanding of how interactions between honey bees and other species affect infection outcomes remain. Some of the most prevalent bee pathogens like SBV and to a lesser degree DWV were affected by ant presence. Yet, these pathogens coinfect bees together with a range of other pathogens and their interactions within the host are not well known. Stress through invasive ants may be associated with increases in some viruses but not others. There is limited evidence for Argentine ants actively transmitting viral pathogens, especially since SBV and ToRSV have never been detected in ants, although, their presence affected viral titres regardless. Ants may act as a stressor and thereby cause elevated SBV levels and transcriptomic changes that overlap with changes induced by viral infections. Stress through pesticides, poor nutrition and overcrowded apiaries reduce the bees' immune function (Goulson et al. 2015), which can change covert viral infections already present in the hive to an overt infection (Di Prisco et al. 2013). The effects of stressors, such as ants, may not be apparent at first but have long-term effects on virus communities in bees. 


\section{Chapter 5:RNA interference, used to inhibit immune function, was not effective in controlling Argentine ants in apiaries}

\subsection{Abstract}

New control methods for pest insects are needed as many conventional insecticides are harmful to beneficial insects, humans, and the environment. RNA interference (RNAi) is a cellular mechanism that suppresses targeted gene expression, which could be exploited to offer a safer, environmentally-friendly pest control strategy. Invasive Argentine ants can harm native invertebrates and are a common pest in honey bee apiaries. In this study, we tested whether Argentine ant gene-specific double-stranded RNA (dsRNA) could be used to control Argentine ants in apiaries. Our goal was to use RNAi to increase ant susceptibility to pathogens by silencing two immune-related genes, dicer-1 and spätzle. In a controlled experiment, we applied dsRNA bait in an apiary that had a known ant incursion and compared its effectiveness with a conventional pesticide that contains fipronil. We then observed the effects of dsRNA on expression in immune-related genes, pathogen levels, Argentine ant numbers and beehive survival. No consistent suppression of dicer-1 and spätzle was achieved. However, pathogen recognition receptor, antimicrobial peptide, and immune deficiency (Imd) signalling pathway gene expression were affected by the dsRNA treatment. Yet, this effect was dependent on the sampling day. To determine pathogen infections in Argentine ants, we monitored 15 viruses and four bacterial species. The observed changes in immune genes expression from gene silencing did not lead to an increase in pathogens or a reduction in ant numbers. Many bee colonies in our test site abandoned their hives (absconded) in the control and dsRNA treatments. Only conventional ant control with fipronil significantly reduced ant numbers and increased bee survival. The dsRNA baits that we applied did not appear to be effective in raising pathogen abundance, nor did it result in any control of ants. While further improvements need to be made to develop effective RNAi for ant control, insights into ant immunity and antbee interactions will aid the development of control agents. 


\subsection{Introduction}

Insect pest control is a challenging task as many pesticides are not species-specific and kill a broad range of insects, including beneficial taxa. The increased use of pesticides and insecticides has been identified as one of the main drivers of insect population declines worldwide (Goulson et al. 2015, Sánchez-Bayo and Wyckhuys 2019, van Klink et al. 2020). Neonicotinoids are a group of neuro-active insecticides applied for crop protection. Application of neonicotinoids has recently been banned by the European Union because of their harmful effects on bees and other non-target arthropods (Blacquiere et al. 2012, Di Prisco et al. 2013, Gibbons et al. 2015, Hallmann et al. 2017) but are still widely used in other parts of the world. Other commonly used insecticides, such as fipronil, can cause high mortality in pollinators including honey bees and are harmful to vertebrate life (Gibbons et al. 2015, Pisa et al. 2015). Effects of pesticides on social bees may be unapparent at first because foragers only ingest small amounts of the nectar or pollen they collect from treated plants. Yet, residues that are taken into the hive expose bees to sub-lethal doses of the pesticide over an extended period, which can cause impaired learning and weaken the colony (Henry et al. 2012, O'Neal et al. 2018). Global food production equally depends on controlling insect pests for crop protection while protecting beneficial insects for pollination services (Oerke and Dehne 2004, Klein et al. 2007). Many insecticides used today affect both pests and beneficial insects alike. Speciesspecific and environmentally-friendly pest control is urgently needed as insect populations around the world are declining at unprecedented levels (Biesmeijer et al. 2006, Potts et al. 2010, Hallmann et al. 2017, Goulson 2019).

Genetic advancements have the potential to revolutionise pest control (Baum et al. 2007). Genetic modification of pests allows for target-specific control, however, altering genes is still highly debated and not always culturally accepted (Lucht 2015, Palmer and Mercier 2020, Ichim 2021). Non-transformative alternatives such as RNA interference (RNAi) are considered safer and have the potential to deliver the same targeted species-specific effects (Whyard et al. 2009). RNA interference is a conserved cellular gene regulation mechanism that has been widely used to study gene function by silencing specific genes (reviewed by Hannon 2002). Thereby double-stranded RNA (dsRNA) is introduced into target cells, which is then processed into 21 to 24 nucleotide long small RNAs by the dicer RNase III enzymes. These small RNAs are then incorporated into the RNA-induced silencing complex (RISC) and target complementary messenger RNA (mRNA) to exert post-transcriptional gene silencing (Hannon 2002). Through this process, the expression of specific genes can be silenced without altering 
the genomic DNA. With decreasing costs of dsRNA production, RNAi could soon become widely available for pest control (Burand and Hunter 2013, Mat Jalaluddin et al. 2019). Recent uses of dsRNA for pest control include the treatment of breeding waters of Aedes mosquito larvae to reduce mosquitos vectoring human pathogens (Lopez et al. 2019) and the control of honey bee pests such as Varroa mites or fungal and viral diseases (Hunter et al. 2010, Garbian et al. 2012, Leonard et al. 2020). The potential uses of RNAi are manifold, and more research is needed to explore applications.

RNA interference could aid the control of invasive ants. Invasive ant species are a common apiary pest (Payne et al. 2020) but also outcompete native ants and prey on invertebrate fauna (Holway 1999, Wilder et al. 2013, Garnas et al. 2014). The invasive Argentine ant (Linepithema humile) has been shown to disrupt flower visitation by pollinators (Sinu et al. 2017) and to enter beehives to rob food stores and prey on bee brood, which can cause bee colonies to abscond and abandon their hive (Payne et al. 2020). Controlling pests that closely interact with honey bees is challenging as it requires species-specific agents that do not harm bees. Furthermore, the formation of ant supercolonies, which is regarded as one of the key factors that determine the success of invasive ants (Holway et al. 2002), often undermines conventional pest control methods. Argentine ant supercolonies consist of large networks of cooperating nests (Tsutsui et al. 2000, Tsutsui and Case 2001, Suarez et al. 2002), which make long-term control difficult when lost queens and workers are easily replaced from neighbouring nests. An evaluation of invasive ant eradication attempts concluded that the majority of attempts to eradicate Argentine ants have failed, especially when using fipronil (Hoffmann et al. 2016).

Another challenge in ant control is that some invasive ant spceis can undergo boombust dynamics, whereby large ant incursions virtually disappear within a short time (Wetterer et al. 2006, Cooling et al. 2012, Gruber et al. 2013, Lester and Gruber 2016). Pathogens have been hypothesised as a likely cause of these dynamics (Simberloff and Gibbons 2004, Lester and Gruber 2016). Argentine ants harbour a diverse range of viruses (Gruber et al. 2017, Viljakainen et al. 2018, Felden et al. 2019). Many of which have only recently been discovered and their effects on ants are unknown (Baty et al. 2020). Viral pathogens could be harnessed for population control of invasive ants.

Parasites may be lost during introduction leading to greater parasite species richness in native compared to introduced populations (Torchin et al. 2003). A release from predators or 
parasites is hypothesised to be a key factor in invasion success (Keane and Crawley 2002) as enemies are important in host populations dynamics. In invasive species that have lost some of their pathogens during the invasion event (enemy release), the introduction of pathogens from their native range is regarded as an option to control populations (Hajek and Eilenberg 2018). Microbial symbionts and viruses in Argentine ants have undergone a series of bottlenecks along the invasion pathway leading to reduced diversity in New Zealand's ant population (Lester et al. 2017, Felden et al. 2019). Yet, boom-bust dynamics suggest that pathogens exist within introduced ranges. Invasive ant supercolonies could even facilitate pathogen transmission as cooperating nests hinder social barriers (Nunn et al. 2015) that are part of behavioural responses that function as the society's immune system (Cremer and Sixt 2009). Reduced genetic diversity following an invasion bottleneck may adversely affect the efficiency of behavioural and cellular immune responses (Ugelvig et al. 2010, Simone-Finstrom et al. 2016), therefore pathogen outbreaks appear likely. On the other hand, social structures could allow invasive ants to tolerate high pathogen loads (Cremer 2019), which would hinder population control through pathogens.

We propose a new avenue for Argentine ant control aiming to induce an outbreak of a pathogen that is already present within the population, by suppressing the ants' immune system through RNAi (Figure 5.1). Immunosuppression could be achieved by targeting immune genes using dsRNA. Bait with dsRNA can be brought into the ant nest by foragers and then fed to nestmates via trophallaxis (mouth-to-mouth sharing of food between nestmates), potentially allowing for RNAi in colony members that may not actively forage, such as nurses and queens (A and B in Figure 5.1). To achieve a long-lasting effect, the pathogen needs to easily spread between immunosuppressed colony members and to healthy nestmates and neighbouring ant nests ( $\mathrm{C}$ and $\mathrm{D}$ in Figure 5.1). The supercolonial behaviour of Argentine ants, wherein individual ants can move freely between many different nests and colonies, might facilitate pathogen spread within infestations. To test this novel approach, dsRNA targeting the ants' immune responses were first tested in laboratory experiments to establish whether gene immune gene expression can be reduced using dsRNA (Felden et al. in preparation) and then whether this approach would be feasible for ant control in the field (this study). The Argentine ant genome contains RNAi machinery genes (Smith et al. 2011) which suggest that gene silencing through RNAi is feasible. Three RNA pathways are involved in RNA processing with different cellular and immune functions. The micro RNA (miRNA) and the small interfering RNA (siRNA) pathways have primarily housekeeping and antiviral functions and are mediated 
through dicer-1 and dicer-2, respectively (Lee et al. 2004). Most arthropods possess two dicer genes from both dicer subfamilies (Gao et al. 2014, Dowling et al. 2016). The piwi-interacting RNA pathway is mostly involved in the silencing of transposable elements.

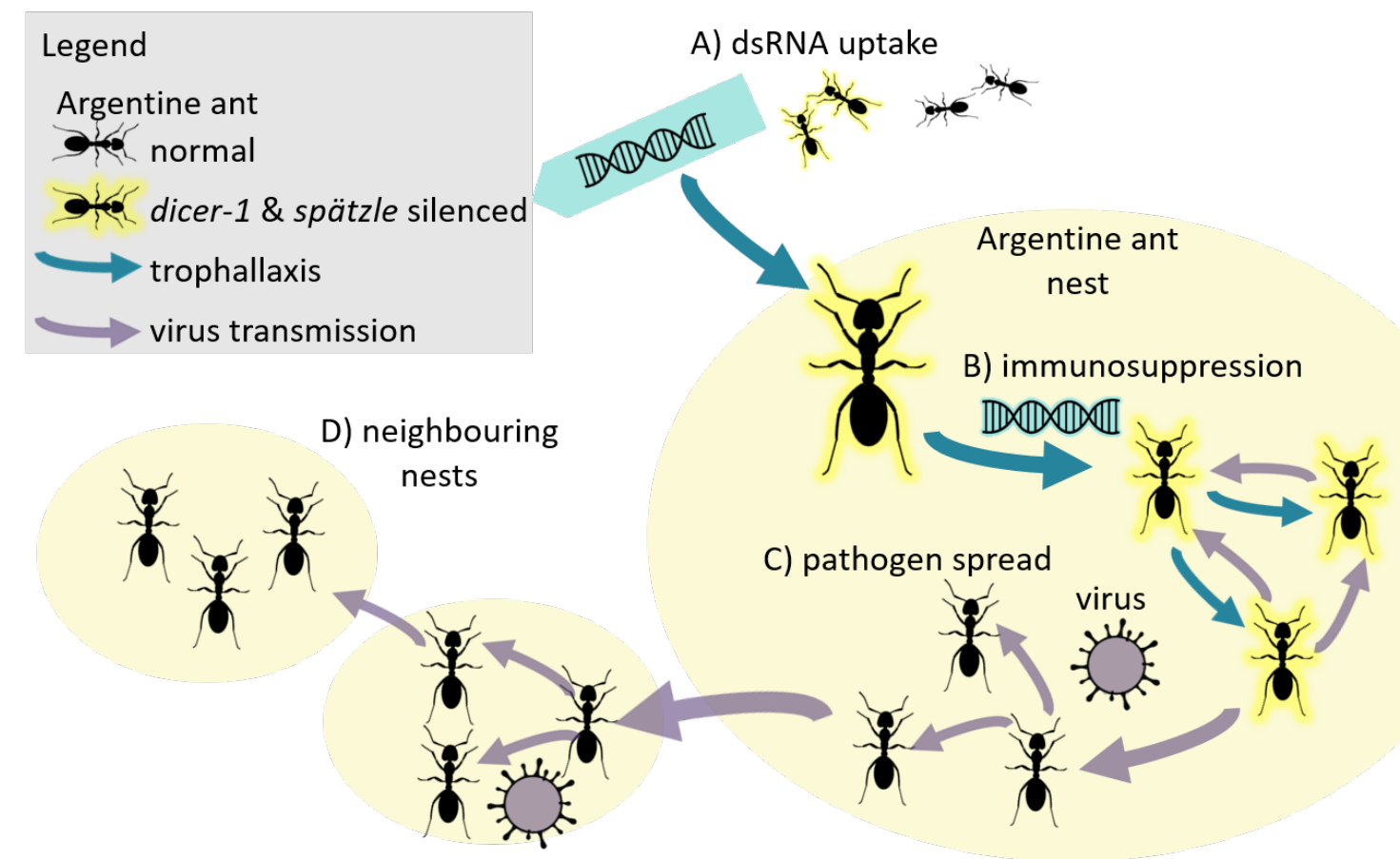

Figure 5.1: Schematic overview of the hypothesised method to use RNA interference (RNAi) induced immunosuppression for Argentine ant pest control. A: Argentine ants feed on bait containing double-stranded RNA (dsRNA) dicer-1 and dsRNA spätzle that reduces dicer-1 and spätzle expression (yellow glow). B: The dsRNA bait is spread to nestmates by sharing dsRNA bait through trophallaxis. C: Reduced immune gene expression suppresses immune function causing ants to become hyper susceptible to pathogens that are already present in the nest. Pathogen transmission is facilitated through highly infected immunosuppressed colony members. D: Pathogens are spread to neighbouring nests with the potential to induce a pathogen epidemic within the ant population.

RNA interference is the key antiviral defence mechanism in insects (van Rij et al. 2006), but other pathways such as JAK-STAT, Toll, Immune deficiency (Imd), autophagy, eicosanoid and endocytosis can also be activated upon viral encounter (Ding 2010, Kingsolver et al. 2013). Ant control was tested by targeting the RNAi and Toll signalling pathways. As part of the RNAi pathway dicer-2 recognises virus-derived dsRNA. Silencing of dicer-2 and argonaute-2, which closely interacts with dicer-2, has shown to increase susceptibility to +ssRNA and dsRNA viruses in Drosophila (van Rij et al. 2006, Zambon et al. 2006). Although, another study that silenced dicer-2 in black-faced leafhoppers reported increased mortality with no effect on virus acquisition or transmission (Chen et al. 2015), suggesting that effects could be host- or virus-specific. Various studies have reported the effects of silencing Toll-pathway genes on pathogen susceptibility. The Toll signalling pathway named after the Toll receptors 
that recognise pathogen-associated molecular patterns is largely involved in antibacterial and antifungal defences (Lemaitre et al. 1997). Silencing toll4 in a shrimp species increases viral replication levels ( $\mathrm{Li}$ et al. 2018) and silencing of dorsal-1A, which is activated by Toll, increases DWV titres in honey bees (Nazzi et al. 2012). But also anti-bacterial and anti-fungal defences are affected. For instance, silencing of Myeloid differentiation primary response 88 (Myd88), an adaptor protein used by Toll, increases susceptibility to a fungal pathogen in locust species (Zheng et al. 2020). TmToll-7 silenced mealworm beetles show reduced survival to Escherichia coli infections (Park et al. 2019) and TmSPz6 (spätzle) silenced beetles show increases susceptibility to E. coli and Staphylococcus aureus (Edosa et al. 2020). Spätzle is a receptor-ligand that enhances cactus and Toll and could provide a suitable target for immunosuppression in Argentine ant control. In insect pest control, targeting the cactus gene in the Toll signalling pathway has proven as a sensitive RNAi target, however, with lethal effects (Ulrich et al. 2015, Bingsohn et al. 2017).

Feeding experiments using dsRNA targeting dicer-1 and spätzle have successfully reduced expression in laboratory trials (Felden et al. in preparation). Here, we ask whether dsRNA application in the field reduces dicer-1 and spätzle expression in Argentine ants. By silencing the expression of these genes, we expect to disrupt the immune system of Argentine ants to increase their susceptibility to one or multiple pathogens that are already present in the ant population. Distinct responses to different classes of pathogens in Argentine ants suggest that disrupting signalling pathways could affect pathogen dynamics (Lester et al. 2019). This immunosuppression could then facilitate pathogen transmission so that a large proportion of the colony falls sick and ant numbers can be reduced. We used quantitative PCR (qPCR) to investigate whether the expression of immune genes or pathogen levels in Argentine ants were affected by dsRNA application. Because Argentine ants can be an apiary pest (Payne et al. 2020), we further tested whether Argentine ant control through dsRNA is feasible near beehives and hypothesised that controlling ants can improve the survival of bee colonies.

\subsection{Materials and methods}

\subsubsection{Double-stranded RNA bait and experimental design}

Double-stranded RNA oligonucleotides were designed to target mRNAs of two candidate genes dicer-1 (LOC105668788, XM_012361369.1) and spätzle (LOC105678357, XM_012377618.1 and XM_012377620.1, Table S5.1) to achieve targeted RNAi-mediated gene silencing through dsRNA feeding (Felden et al. in preparation). Gene annotations are 
based on Gnomon predictions using the NCBI Reference Sequence NW_012160832.1. Double-stranded RNA dicer-1 (495 bp) and spätzle (500 bp) were produced by agroRNA (Seoul, South Korea). Suppression of these candidate genes through dsRNA had been achieved in lab experiments testing short- (two days) and long-term (28 days) effects of feeding ants with a sugar dsRNA mix (Felden et al. in preparation). Briefly, groups of 250 worker ants and one queen were fed the equivalent of $0.2 \mu \mathrm{L}$ dsRNA/sugar mix per ant every $12 \mathrm{~h}$ over two days To test the effects of a longer exposure to dsRNA that would be closer to dsRNA treatment for population control experimental colonies of approx. 900 workers and one queen were fed $200 \mu \mathrm{L}$ sugar/dsRNA solution every day over 28 days (Felden et al. in preparation). These lab trials showed that RNAi mediated immune gene knock-down was feasible in Argentine ants as a 50\% decrease in spätzle expression was observed after two and seven days of treatment and a $35 \%$ decrease up until the end of the 28-day treatment period. Similarly, dicer- 1 expression had been reduced, however not as severely (not more than 30\% decrease) and for a shorter period only until seven days of dsRNA feeding (Felden et al. in preparation). Hence, dicer-1 and spätzle provided suitable candidates for testing RNAi in the field.

As a test site for Argentine ant control, we used an agricultural pasture site (-34.912493, 173.331153) that had a known Argentine ant incursion on the Karikari peninsula in the north of New Zealand. Although Argentine ants within New Zealand belong to the same supercolony (Corin et al. 2007) and were expected to structure into cooperating nests that spanned the entire site, the foraging range of Argentine ants is below $36 \mathrm{~m}$ (Hogg et al. 2018), which allowed us to test different treatments within the same site. The abundance of ants was established through baiting by using cotton soaked in sugar water that was laid out in a grid around the site and counting ant numbers after 30 minutes. Based on these counts, we decided to choose a transect parallel to a line of pine trees that bordered the north-western side of the site (B in Figure 5.3). Fifteen bait stations for the experiment were established along this transect, every $40 \mathrm{~m}$ apart to avoid overlap from foraging ants (B in Figure 5.3).

Each station was randomly assigned to one of three treatment groups: 1) dsRNA in sugar water, 2) sugar water only as a control and 3) Vanquish ${ }^{\mathrm{TM}}$ Argentine Ant Bait (Bait Technology Ltd, New Zealand; hereafter vanquish bait) (Figure 5.2). Each of the 15 treatment stations consisted of four cardboard 'shelters' (A in Figure 5.3). In each we placed either two tubes with dsRNA (dsRNA treatment), $3 \mathrm{~mL}$ dsRNA dicer-1 and $3 \mathrm{~mL}$ dsRNA spätzle (18 mg of each dsRNA at each treatment station), two tubes containing $3 \mathrm{~mL}$ sugar bait each (control treatment), or approximately $3 \mathrm{~cm}$ of vanquish bait (vanquish treatment) (Figure 5.2). As a bait 
for Argentine ants, dsRNA was diluted to $1.5 \mathrm{mg} / \mathrm{mL}$ in $300 \mathrm{mM}$ sugar water, which was established as an attractive bait in preliminary experiments (Felden et al. in preparation) and the same sugar concentration was used in the control treatment. Tubes for the dsRNA and sugar treatment were loosely closed with a cotton ball that allowed ants to enter the tube but prevented larger insects like bees from consuming the bait. Vanquish bait is a green paste that contains fipronil $(0.01 \%$ weight) which is a slow-acting broad-spectrum insecticide that disrupts the nervous system (Jackson et al. 2009). During the treatment period, each station in the dsRNA and sugar control group was supplied with $24 \mathrm{~mL}$ bait daily, while Vanquish bait was applied on days one, two, five, and ten.

A. Bait stations setup

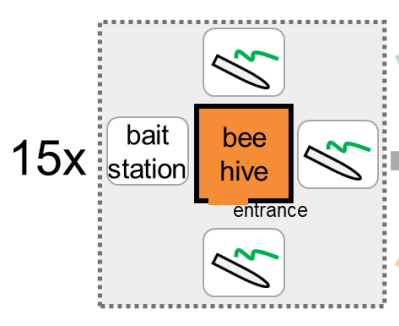

B. Treatments dsRNA $(1.5 \mathrm{mg} / \mathrm{mL}$ in $300 \mathrm{mM}$ sugar water)

$$
5 x
$$<smiles>[13CH3]</smiles>

C. Sampling and analysis

\section{Abundance $(\square \bigcirc \nabla)$}

$\checkmark$ Sugar bait around hives, day 1-17 Estimating ant numbers from hive photos, day 1-17

Gene expression \& pathogen levels $(\square \bigcirc)$

$\checkmark$ day 5 \& 8 (during treatment)

$\checkmark$ day $10 \& 12$ (post treatment)

Figure 5.2: Experimental design to test double-stranded RNA (dsRNA) for ant population control. A: 15 beehives were moved into a field site with a known Argentine ant incursion. Bait stations were established around all four sites of each hive. B: One out of three treatments was randomly assigned to each hive and bait stations were supplied with dsRNA targeting dicer-1 and spätzle (green), a sugar control (purple) or a commercial ant bait containing fipronil (orange). C: Ant abundance around beehives/bait stations was established every second day by placing a sugar bait in a grid around the hive and using photos of each side of the hive box to count ant numbers (all treatments). Ants treated with dsRNA (green) and the sugar control (purple) were collected during the treatment (day 5 and 8) and after the treatment (day 10 and 12) to study gene expression and pathogen levels using quantitative PCR. 


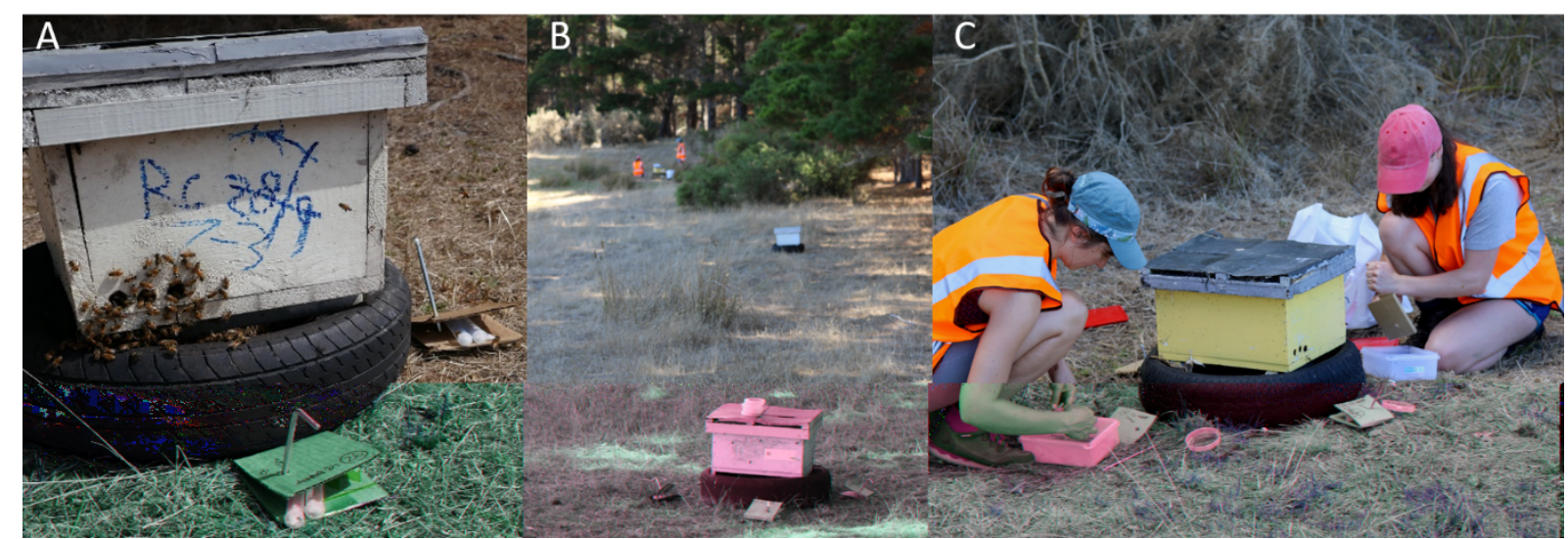

Figure 5.3: Experimental setup and Argentine ant (L. humile) sampling. A: Ant bait was set up in four treatment stations around each beehive. Two tubes with dsRNA or control sugar mix were closed with a cotton ball and placed under a cardboard shelter fixated with a peg. B: Beehives and treatment stations around them were set up in a transect along the pine trees bordering the site. Each hive was $40 \mathrm{~m}$ apart from the next one. C: Argentine ants were sampled by carefully transferring them from the cardboard shelters into Fluon ${ }^{\circledR}$ coated plastic boxes. Photo A by Jana Dobelmann, B and C by Phil Lester.

Argentine ants are a pest that can be a major source of hive loss in apiaries in the Northland region of New Zealand (Brown et al. 2018). Our study site had previously been used as an apiary before hive losses due to ants forced beekeepers to abandon the site. To test the feasibility of Argentine ant control in an apiary, we placed one bee colony in the centre of each treatment station (A in Figure 5.3). Beehives were small nucleus colonies of about the same age that were provided by a commercial beekeeping operator (Haines Apiaries, Kaitaia) and placed on tyres with their entrance facing away from the pine trees. Beehives were moved into the site on day zero (19/02/20). Ant treatment (dsRNA and control) started on day one (20/02/20) and continued for eight days (until 27/02/20) after which no more bait was applied. Observations continued until day 17 (06/03/20).

\subsubsection{RNA extraction and reverse transcription quantitative PCR}

Samples of 100 Argentine ants were collected for molecular analyses from the dsRNA and control group on days five, eight, ten, and twelve (C in Figure 5.3). We also collected ants on days zero and three, however, the cooling system (dry shipper) failed and samples could not be used in further steps as RNA quickly degrades at room temperature. No ants were collected from vanquish bait controlled ants, as this treatment served as a positive control to reduce ant numbers and we were only interested in the effects of dsRNA on gene expression and pathogen levels. The resulting 40 samples of 100 ants were washed three times with ice-cold phosphatebuffered saline (PBS, Ambion, Texas, USA) before RNA extraction in order to remove 
potential pathogens on the exoskeleton of the ants. Then $500 \mu \mathrm{L}$ TRIzol ${ }^{\circledR}$ Reagent (Life Technologies, California, USA) was added and ants were homogenised at $4^{\circ} \mathrm{C}$ with a bead mill homogeniser (Precellys Evolution, Bertin Technologies, France) using $0.5 \mathrm{~mm}$ steel beads with two intervals of $20 \mathrm{~s}$ at $6800 \mathrm{rpm}$ and a $30 \mathrm{~s}$ pause. We mixed $450 \mu \mathrm{L}$ of the homogenate with $450 \mu \mathrm{L} 100 \%$ EtOH and extracted RNA using the Direct-zol RNA Miniprep Plus Kit (Zymo Research, California, USA) following the manufacturer's instructions and including the incolumn DNase treatment. RNA quantity and quality were checked using a Nano Photometer ${ }^{\circledR}$ (NP80, Implen, Germany).

TaqMan Array Micro Fluidic Cards (Applied Biosystems, ThermoFisher Scientific, California, USA) containing 47 custom TaqMan assays and one mandatory manufacturing control assay (see Table S5.2 for TaqMan assay sequences), were used to measure gene expression and pathogen levels in Argentine ants using quantitative PCR (qPCR). We used three genes as endogenous controls (peptidyl-prolyl cis-trans isomerase like (PPI), dynactin, Fip-1). Targeted genes were mostly associated with the immune system and included five targets in the RNAi pathway, three in the JAK-STAT pathway, five in the Toll pathway, two in the Imd/ c-Jun N-terminal kinases (JNK) pathway, two antimicrobial peptides and four recognition receptors. Because RNAi could directly or indirectly affect metabolic genes through pathogen interactions (Lee and Lee 2018), we also targeted three metabolic genes. To see whether dsRNA and potentially reduced immune function affects ant pathogen communities, we measured pathogen loads for four bacteria, four bee-associated viruses, eleven ant-associated viruses and a putative bacteriophage (details in Table S5.2).

Primers for TaqMan assays were designed using Primer Express software v3.0.1 (Applied Biosystems, ThermoFisher Scientific, California, USA) except those for black queen cell virus (BQCV), which were modified from Chantawannakul et al. (2006). Twenty-seven of the newly designed assays, including three reference genes that were critical for the array, were tested on pooled Argentine ant complementary DNA (cDNA) to confirm amplification (assays indicated in Table S5.2; qPCR data not shown). Briefly, SuperScript IV (Invitrogen, ThermoFisher Scientific, Lithuania) was used to generate cDNA from $2 \mu \mathrm{L}$ pooled Argentine ant RNA in $20 \mu \mathrm{L}$ reactions. Quantitative PCR was conducted with $80 \mathrm{ng}$ of cDNA mixed with TaqMan Fast Advanced Master Mix (Applied Biosystems, ThermoFisher Scientific, USA) and the TaqMan assay ( $20 \mu \mathrm{L}$ total volume). Thermal cycling ran as follows: $50^{\circ} \mathrm{C}$ for $2 \mathrm{~min}, 95^{\circ} \mathrm{C}$ for $2 \mathrm{~min}$, followed by 40 cycles of $95^{\circ} \mathrm{C}$ for $1 \mathrm{~s}$ then $60^{\circ} \mathrm{C}$ for $20 \mathrm{~s}$ on a QuantStudio 7 RealTime PCR System (Applied Biosystems, ThermoFisher Scientific, California, USA). 
From the 40 samples collected during the experiment, approximately $800 \mathrm{ng}$ of RNA was reverse transcribed into cDNA using the Superscript IV reverse transcription kit following the manufacturer's instructions and added to the TaqMan Fast Advanced Master Mix for qPCR. Reactions were carried out with a final concentration of $2 \mathrm{ng} / \mu \mathrm{L}$ (400 ng) cDNA, which was loaded onto custom-designed TaqMan Array Micro Fluidic Cards. Thermal cycling on a QuantStudio 7 Real-Time PCR System was conducted as per the manufacturer's instructions.

Raw qPCR data were analysed using the Thermo Fisher Connect Relative Quantification app v4.3 to obtain Cycle quantification $\left(\mathrm{C}_{\mathrm{q}}\right)$ values that were then exported and analysed in R v4.0.1 (R Core Team 2014). We normalised $\mathrm{C}_{\mathrm{q}}$ values against the mean $\mathrm{C}_{\mathrm{q}}$ of the three reference genes (PPI, Fip-1, and Dynactin) to calculate $\Delta \mathrm{C}_{\mathrm{q}}$ values. The use of these three genes was established as the most stable combination of reference genes using NormFinder software v0.953 (Andersen et al. 2004). For statistical analyses, gene expression values (2-ACq, not normalised against the control group) were used. Gene expression and pathogen load data did not follow a normal distribution and could not be normalized using standard transformation techniques. Therefore, non-parametric tests were used to assess differences between treatment groups. Wilcoxon rank-sum tests for each target (genes and pathogens) within sampling days were used to test for differences between dsRNA treated nests and control nests using $2^{-\Delta \mathrm{Cq}}$ values. In order to visualise fold expression change in the dsRNA treatment group, $\Delta \mathrm{C}_{\mathrm{qT} \text { Treat }}$ values were normalised against the mean $\Delta \mathrm{C}_{\mathrm{q}}$ of the sugar control group $\left(\Delta \mathrm{C}_{\mathrm{q} C r t \mathrm{l}}\right)$ and calculated as $\Delta \Delta \mathrm{C}_{\mathrm{q}}$ values. Fold expression change was then calculated as $2^{-\Delta \Delta \mathrm{Cq}}$ (Livak and Schmittgen 2001) so that values below one indicate negative expression and above one positive expression changes.

\subsubsection{Effects of dsRNA on gene expression and pathogen levels}

Dicer-1 and spätzle gene expression values were analysed using repeated measures permutational multivariate analysis of variance (PERMANOVA). We used two analyses to account for the nested design of our study. Firstly, we analysed temporal differences within nests (within-subjects) and then treatment differences between nests (between-subjects). To analyse effects within nests over time we used the adonis() function in vegan (Oksanen et al. 2017) with 'ant nests' as strata, to account for repeated measures. 'Time', 'treatment', and their interaction were fixed effects and Bray-Curtis distances of dicer-1 or spätzle expression were the response variables. The 'time' main effect and 'interaction' effect were analysed using this method, however, the 'treatment' main effect was analysed in separate PERMANOVA. 'Ant 
nests' were nested within treatments in our experimental design, so that differences between treatments could not be assessed when restricting permutations to nests. Therefore, we used the function nested.npmanova() in the BiodiversityR package (Kindt and Coe 2005) to test for the 'treatment' main effect in a second analysis. Models were fitted using treatment as the main factor and ant nest as the nested factor with Bray-Curtis distances of gene expression as the response. Both permutational analyses were run with 999 permutations.

To test whether genes other than those targeted with dsRNA and pathogen levels were affected by time or treatment we grouped genes into different immune pathways (RNAi, Toll, Imd/JNK, JAK-STAT, recognition receptors, antimicrobial peptides) or metabolic genes and pathogens into bacteria, ant-associated viruses and bee-associated viruses (Table 5.1). For these nine groups, we calculated distance matrices and used the same repeated measures PERMANOVA analyses as described above. Non-metric multidimensional scaling was used to visualise gene expression and pathogen load data in two-dimensional plots using the metaMDS function that is part of the vegan package. 
Table 5.1: Grouping of genes and pathogens for statistical analyses. Virus abbreviations: Deformed wing virus (DWV), Kashmir bee virus (KBV), black queen cell virus (BQCV), Linepithema humile virus 1 (LHUV1), Linepithema humile C virus 1 (LhuCV1), Linepithema humile bunya-like virus 1 (LhuBLV1), Linepithema humile entomopoxvirus 1 (LhuEV1), Linepithema humile partiti-like virus 1 (LhuPLV1), Linepithema humile polycipivirus 2 (LhuPcV2), Linepithema humile picorna-like virus 1 (LhuPiLV1), Linepithema humile totilike virus 1 (LhuTLV1). Gene acronyms: myeloid differentiation primary response protein MyD88-like (MyD88), dredd, fas-associated death domain protein (Fadd), tyrosine-protein kinase hopscotch (hopscrotch), signal transducer and activator of transcription 5B (Stat5B), domeless.

\begin{tabular}{|c|c|c|}
\hline Group & Gene or Pathogen & GenBank accession \\
\hline \multirow{5}{*}{ Immune pathway: Toll (5) } & protein Toll (A) & LOC105680007 \\
\hline & protein Toll (B) & LOC105674053 \\
\hline & protein spätzle & LOC105678357 \\
\hline & embryonic polarity protein dorsal & LOC105677755 \\
\hline & MyD88 & LOC105677438 \\
\hline \multirow{5}{*}{ Immune pathway: RNAi (5) } & endoribonuclease dcr-1 & LOC105668788 \\
\hline & endoribonuclease dicer & LOC105670277 \\
\hline & protein argonaute-2 (A) & LOC105677214 \\
\hline & protein argonaute-2 (B) & LOC105669925 \\
\hline & protein argonaute-3 & LOC105679318 \\
\hline \multirow{2}{*}{$\begin{array}{l}\text { Immune pathway: } \\
\text { Antimicrobial peptide (2) }\end{array}$} & defensin-2 & LOC105675717 \\
\hline & hymenoptaecin & LOC105670591 \\
\hline \multirow{2}{*}{$\begin{array}{l}\text { Immune pathway: Imd/ JNK } \\
\text { (2) }\end{array}$} & dredd & LOC105678455 \\
\hline & Fadd & LOC105676271 \\
\hline \multirow{3}{*}{ Immune pathway: JKST (3) } & hopscotch & LOC105677920 \\
\hline & Stat5B & LOC105672556 \\
\hline & domeless & LOC105676117 \\
\hline \multirow{4}{*}{$\begin{array}{l}\text { Immune pathway: pathogen } \\
\text { recognition (4) }\end{array}$} & beta-1,3-glucan-binding protein-like (A) & LOC105673881 \\
\hline & beta-1,3-glucan-binding protein-like (B) & LOC105674418 \\
\hline & peptidoglycan-recognition protein LC-like & LOC105678063 \\
\hline & peptidoglycan recognition protein-like & LOC105672853 \\
\hline \multirow{3}{*}{ Metabolic genes (3) } & cytochrome b-c1 complex subunit 2 & LOC105670362 \\
\hline & fatty acid synthase-like & LOC105679506 \\
\hline & guanylate kinase & LOC105674201 \\
\hline \multirow{3}{*}{ Bee viruses (3) } & DWV & NC_004830 \\
\hline & KBV & NC_004807 \\
\hline & BQCV & MF417634 \\
\hline \multirow{8}{*}{ Ant viruses (8) } & LHUV1 & NC 003784 \\
\hline & LhuCV1 & MH213244 \\
\hline & LhuBLV1 & MH213237 \\
\hline & LhuEV1 & MH213250 \\
\hline & LhuPLV1 & MH213239- 240 \\
\hline & LhuPcV2 & MH213248 \\
\hline & LhuPiLV1 & MH213235 \\
\hline & LhuTLV1 & MH213243 \\
\hline \multirow{4}{*}{ Bacteria (4) } & Pseudomonas spp. & MH997630.1 \\
\hline & Lactobacillus florum & AB498046.1 \\
\hline & Serratia proteamaculans & NR 025341.1 \\
\hline & Acinetobacter spp. & AJ303011.1 \\
\hline
\end{tabular}




\subsubsection{Effects of dsRNA and vanquish bait on ant numbers and hive survival}

Argentine ant numbers were assessed every second day starting from day zero until day 17 by laying out sugar bait around the fifteen hives/treatment stations. At each treatment station with a beehive in its centre, eight cotton balls soaked in 300nM sugar water were placed in a three by three grid with the centre point being the beehive and every cotton ball one metre apart from the next one. After 30 minutes the number of ants on the cotton was counted by carefully transferring the cotton ball into a plastic box lined with Fluon ${ }^{\circledR}$ (BioQuip, USA) to prevent ants from escaping and using a click counter. Beehives were visually inspected every day. We took photos of all four sites of each hive every second day and noted when bees absconded (abandoned their hive). The number of ants and bees present on the outside of the hive was estimated from photos using ImageJ (Schindelin et al. 2015). We expected that bees on the outside of the hive (except on the side with the hive entrance), could indicate defensive behaviour towards ants or attempts to seal off gaps which ants were using to access the hive. Kaplan-Meier survival curves for beehive survival were generated and analysed using the R package survival (Therneau 2020) and visualised using the survminer package (Kassambara et al. 2017). Multiple comparisons between treatment groups were calculated using pairwise_survdiff() and $p$-values were adjusted for multiple testing using the BenjaminiHochberg procedure (Benjamini and Hochberg 1995).

We used generalised linear mixed-effects models to assess whether Argentine ant numbers on baits or Argentine ant numbers on beehives were affected by treatments. The first model used ant numbers on baits as the dependent variable, with treatment, time and their interaction included as fixed effects. The treatment station around which the bait counts were conducted was included as a random effect. In the second model ant numbers on beehives was the dependent variable and we initially fitted a model with treatment, time, the number of bees on the outside of the hive and the mean ant number on baits around the hive as fixed effects with hive as the random effect. Models were built in R v4.0.1. using the lme4 package (glmer and glmer.nb function). We used the function overdisp.glmer() in the RVAideMemoire package (Hervé 2018) to evaluate overdispersion and used negative binomial distributions when Poisson models were overdispersed. The best model was chosen by fitting the full model with all predictors and removing fixed effects in a stepwise manner if removal lowered the corrected Akaike information criterion (AICc). 


\subsection{Results}

\subsubsection{Gene expression in Argentine ants treated with dsRNA}

Our first aim was to suppress the expression of dicer-1 and spätzle using dsRNA. Commonly, a large number of ants could be seen within the tubes drinking the bait soon after its introduction. Tubes with dsRNA bait or sugar bait were mostly empty after one day and no preference for either of the dsRNA baits was observed by the ants. Expression of dicer-1 and spätzle was not consistently reduced by dsRNA during or after the treatment (Figure 5.4). Gene expression was not affected by treatment, experimental phase, nor their interaction for dicer-1 (Treatment: $\mathrm{F}=0.019, p=0.340$; Time: $\mathrm{F}=1.156, p=0.343$; Treatment $\mathrm{x}$ Time: $\mathrm{F}=1.285, p$ $=0.269$ ) and spätzle (Treatment: $\mathrm{F}=0.232, p=0.792$; Time: $\mathrm{F}=1.726, p=0.138$; Treatment $\mathrm{x}$ Time: $\mathrm{F}=1.332, p=0.246)$.
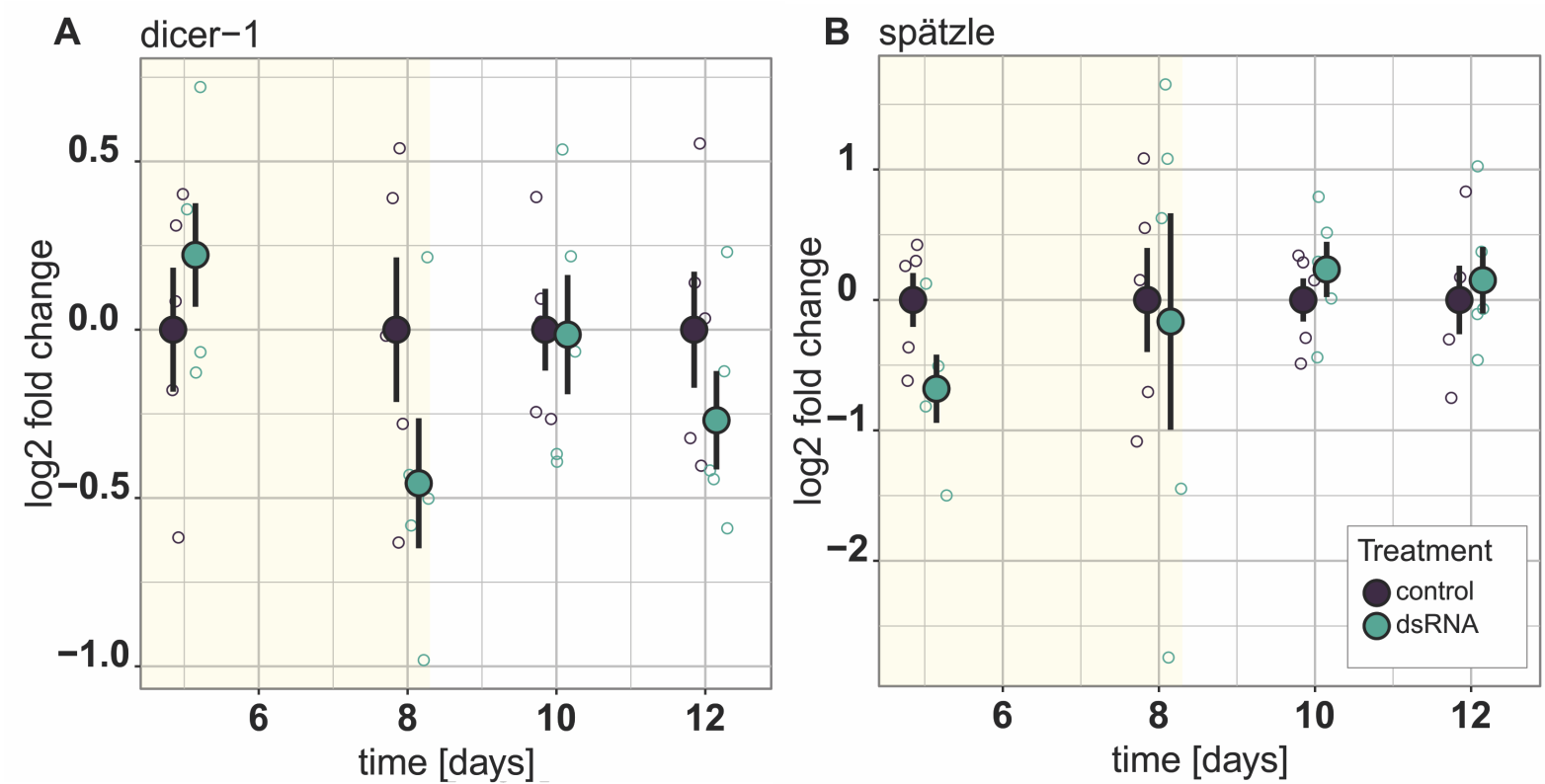

Figure 5.4: Differential gene expression of dicer-1 (A) and spätzle (B) in Argentine ants treated with a mix of double-stranded RNA (dsRNA) targeting dicer-1 and spätzle. Relative gene expression was calculated using the $\Delta \Delta \mathrm{Cq}$ method with dynactin, peptidyl-prolyl cis-trans isomerase like (PPI) and Fip-1 as endogenous control genes and are shown as $\log 22^{-\Delta \Delta \mathrm{Cq}}$ values with negative values indicating lower mRNA amounts compared to the control group. Samples were taken five, eight, ten and 12 days after the start of the experiment. Argentine ants were treated with dsRNA daily from day one until day eight indicated by yellow shading. Fold change gene expression in Argentine ants treated with dsRNA (green) and control (dark purple) is shown as mean \pm standard error (large circle with error bar) with individual data points shown as small circles. 
Next, we aimed to detect dsRNA effects on gene expression in a broader range of immune genes and metabolic genes. The comparison of dsRNA and control within each targeted gene at individual sampling days revealed significant differences in gene expression at day five and eight while the bait was applied (Wilcoxon signed-rank test, Figure 5.5). Significant differences in gene expression were found in hopscotch (mean $2^{-\Delta \Delta \mathrm{Cq}}=1.565, p=$ 0.032 , day 5), fas-associated death domain protein (mean $2^{-\Delta \Delta \mathrm{Cq}}=0.558 p=0.016$, day 8 ), dredd (mean $2^{-\Delta \Delta \mathrm{Cq}}=1.337, p=0.032$, day 5$)$, defensin-2 $\left(\right.$ mean $2^{-\Delta \Delta \mathrm{Cq}}=0.741, p=0.032$, day 8), $\beta$-1,3-GPB-like $A$ (mean $2^{-\Delta \Delta \mathrm{Cq}}=0.321, p=0.032$, day 8 ), argonaute- $2 A$ (mean $2^{-\Delta \Delta \mathrm{Cq}}=$ $0.484, p=0.032$, day 8 ) and fatty-acid-dehydrogenase (mean $2^{-\Delta \Delta \mathrm{Cq}}=0.495, p=0.016$, day 8 ). However, none of these differences was significant when controlling for the false discovery rate (all $p>0.05)$. When using a rank-based test with a small sample size $(\mathrm{n}=10$ at each time point) the lowest $p$-value equals 0.008 (i.e. ranks of one group is always higher than the other such as LhuTLV1 infection loads at day five). Yet, we tested a large number of targets at each time point $(\mathrm{n}=37)$, so that the adjusted $p$-value (Benjamini-Hochberg procedure) equals 0.158 and is considered non-significant. We, therefore, decided to show $p$-values before the adjustment in Figure 5.5 but emphasise that these need to be considered with caution.

When grouping genes into different immune pathways and metabolic genes, expression in all the immune pathways and the metabolic genes group was significantly affected by the sampling day of the experiment (PERMANOVA, Table 5.2 and Figure S5.1). Treatment did not affect gene expression in immune pathway groups or any of the pathogen groups. However, the interaction between time and treatment significantly affected gene expression in pathogen recognition genes, antimicrobial peptides, the Imd/JNK pathway and the genes grouped as metabolic genes (Table 5.2). 

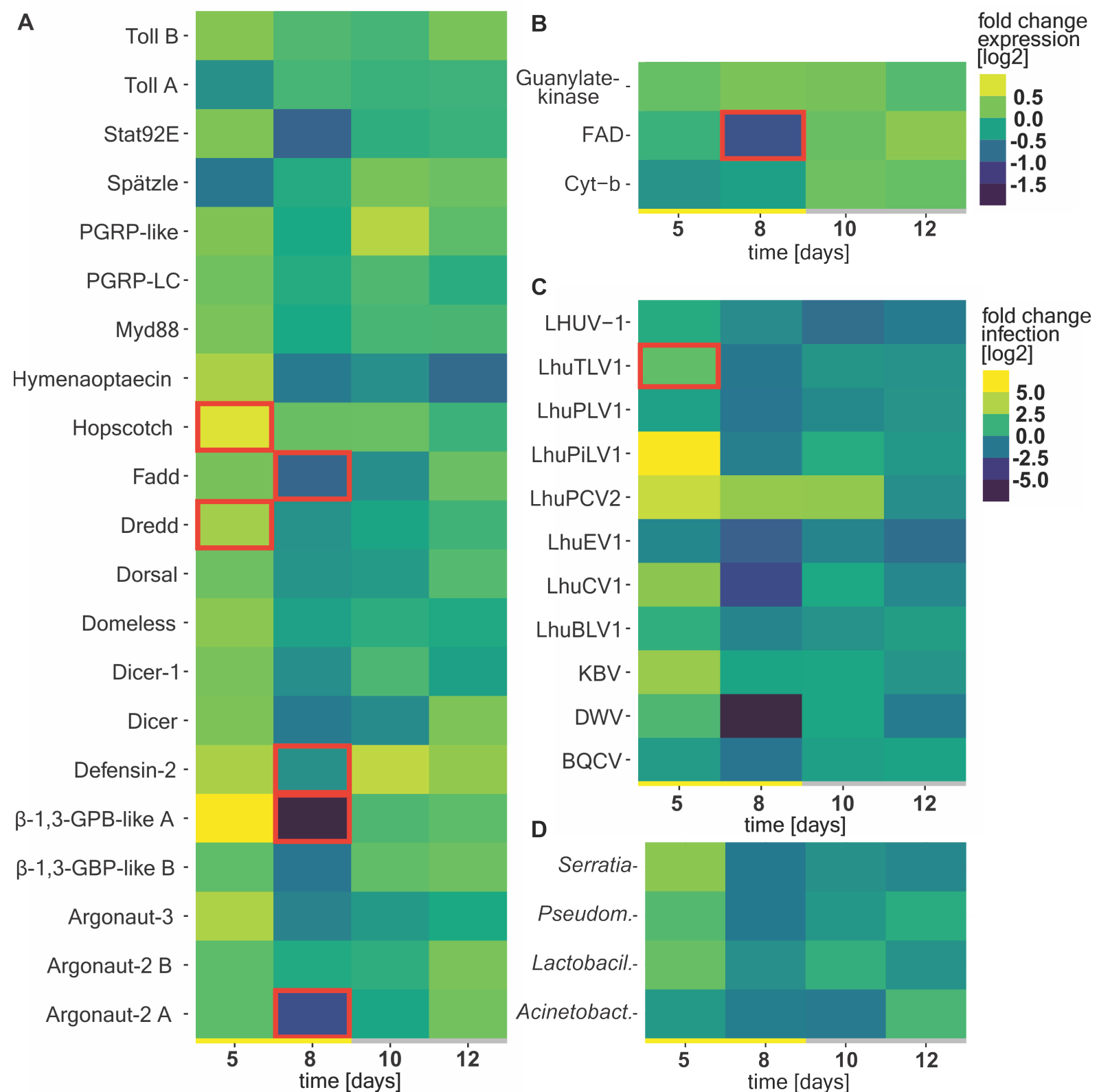

C

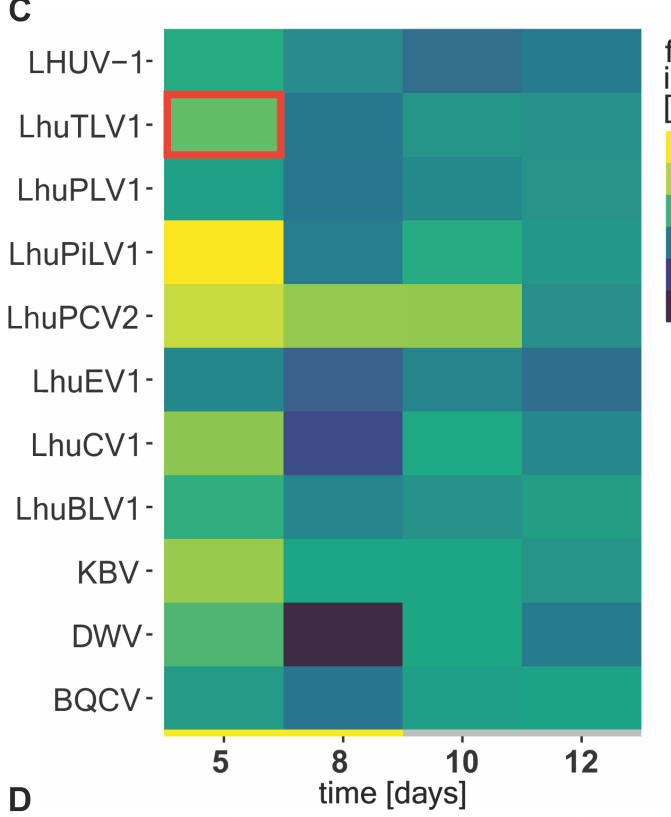

fold change

[log2]

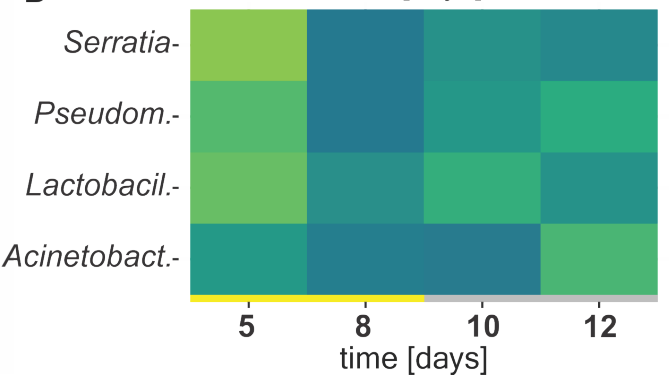

Figure 5.5: A heat map showing gene expression and pathogen load in Argentine ants treated with a mix of double-stranded RNA dicer-1 and spätzle relative to a control group. Yellow indicates increases and dark blue decreases in mRNA levels of immune genes (A), metabolic genes (B), viral load (C) and bacterial load (D). Gene expression (A and B) and pathogen loads (C and D) were calculated using dynactin, peptidyl-prolyl cis-trans isomerase like (PPI) and Fip-1 as endogenous control genes and sugar-treated ants as a control group and are shown as mean $\log 22^{-\Delta \Delta \mathrm{Cq}}$. Note that the "fold change expression" scale belongs to A and B and "fold change infection" to C and D. Red frames indicate significantly different expression $(p<0.05$, Wilcoxon signed-rank test). Daily treatment started from day one until day eight and samples were collected during treatment (day five and eight, yellow bar on x-axis) and after treatment (ten and twelve, grey bar). Virus abbreviations: Linepithema humile virus 1 (LHUV1), Linepithema humile toti-like virus 1 (LhuTLV1), Linepithema humile partiti-like virus 1 (LhuPLV1), Linepithema humile picorna-like virus 1 (LhuPiLV1), Linepithema humile polycipivirus 2 LhuPcV2), Linepithema humile entomopoxvirus 1 (LhuEV1), Linepithema humile $\mathrm{C}$ virus 1 (LhuCV1), Linepithema humile bunya-like virus 1 (LhuBLV1), Kashmir bee virus (KBV), deformed wing virus (DWV), black queen cell virus (BQCV). Bacteria: Pseudomonas spp., Lactobacillus florum, Serratia proteamaculans, Acinetobacter spp. 


\subsubsection{Viral and bacterial pathogen loads in Argentine ants treated with dsRNA}

Most of the pathogens tested were detected in all samples. We found eleven different viruses and four different bacteria in Argentine ants (Figure 5.5). Five viruses were absent from all samples tested: Linepithema humile qinvirus-like virus 1 (LhuQLV1), Linepithema humile polycipivirus 1 (LhuPcV1), Linepithema humile rhabdo-like virus 1 (LhuRLV1), acute bee paralysis virus (ABPV) and pseudomonas phage. Pseudomonas spp. was found in 37 samples, BQCV in 39 samples, and Acinetobacter spp. and Kashmir bee virus (KBV) were only found in 32 out of 40 samples each. All detected pathogens were included in statistical analyses.

The only pathogen significantly affected by the dsRNA treatment was LhuTLV1, which showed significantly higher loads at day 5 (mean $2^{-\Delta \Delta \mathrm{Cq}}=4.208, p=0.008$ ). There was no effect on pathogen levels after treatment stopped. Permutational analyses found that pathogen levels of bee-associated viruses, ant-associated viruses and bacteria were affected by the sampling day of the experiment, but treatment and the interaction between treatment and day did not significantly affect pathogen loads (PERMANOVA, Table 5.2, Figure S5.1).

Table 5.2: Repeated measures permutational multivariate analysis of variance (PERMANOVA) testing the effect of double-stranded RNA, experimental phase and their interaction on gene expression and pathogen loads. Pathogens were grouped into beeassociated viruses, ant-associated viruses and bacteria and genes were grouped into immune pathways (RNA interference (RNAi), pathogen recognition, antimicrobial peptides (AMPs), JAK-STAT, Toll and immune deficiency/ c-Jun N-terminal kinases (Imd/JNK)) and metabolic genes. PERMANOVA were run with 999 permutations and Bray-Curtis dissimilarities. $P$ values $<0.05$ are in bold. Note that the treatment main effect was calculated separately in a nested PERMANOVA to account for the nestedness.

\begin{tabular}{lllllll} 
Effect & Day & & \multicolumn{2}{l}{ Day x Treatment } & \multicolumn{2}{l}{ Treatment } \\
\hline \hline Group & F-value & $p$-value & F-value & $p$-value & F-value & $p$-value \\
\hline bee-associated viruses & 2.377 & $\mathbf{0 . 0 0 6}$ & 0.951 & 0.504 & 0.434 & 0.797 \\
\hline ant-associated viruses & 1.832 & $\mathbf{0 . 0 3 8}$ & 1.367 & 0.133 & 0.488 & 0.864 \\
\hline bacteria & 2.326 & $\mathbf{0 . 0 2 8}$ & 1.197 & 0.310 & 0.699 & 0.551 \\
\hline RNAi pathway & 10.667 & $\mathbf{0 . 0 0 1}$ & 1.618 & 0.169 & 1.385 & 0.271 \\
\hline pathogen recognition & 5.360 & $\mathbf{0 . 0 0 1}$ & 3.164 & $\mathbf{0 . 0 0 8}$ & 0.904 & 0.438 \\
\hline AMPs & 11.649 & $\mathbf{0 . 0 0 1}$ & 3.255 & $\mathbf{0 . 0 3 2}$ & 2.480 & 0.122 \\
\hline JAK-STAT pathway & 3.252 & $\mathbf{0 . 0 0 4}$ & 1.882 & 0.082 & 1.136 & 0.313 \\
\hline Toll pathway & 6.649 & $\mathbf{0 . 0 0 1}$ & 0.691 & 0.599 & 0.555 & 0.658 \\
\hline imd/JNK pathway & 23.515 & $\mathbf{0 . 0 0 1}$ & 3.554 & $\mathbf{0 . 0 2 1}$ & 2.027 & 0.142 \\
\hline metabolic genes & 24.084 & $\mathbf{0 . 0 0 1}$ & 3.160 & $\mathbf{0 . 0 2 9}$ & 2.274 & 0.146 \\
\hline \hline
\end{tabular}




\subsubsection{Effects of dsRNA and vanquish bait on ant numbers}

Poisson distributed mixed-effects models for ant counts on baits and hives were overdispersed and we used a negative binomial distribution. For the number of ants on bait dishes, the full model with sampling day, treatment and their interaction scored the lowest AICc. Sampling day significantly affected ant numbers on baits $(\mathrm{z}=4.896, p<0.001)$. In the dsRNA and control group, we observed an increase in Argentine ant numbers around the bait stations after the start of the experiment when beehives were moved into the site until day 11 or 13, after which numbers slightly decreased towards the end of the experiment (day 17, A in Figure 5.6). This effect was expected as sugar is an attractive bait that can increase ant foraging activity in an area. Ant numbers in the vanquish group decreased after the first treatment and remained low throughout the experiment (A in Figure 5.6). Vanquish bait $(z=-2.884, p=$ $0.004)$ and the interaction between vanquish bait and time $(z=-5.320, p<0.001)$ significantly negatively affected ant numbers, suggesting that over time vanquish bait increasingly reduced ant numbers compared to the other treatments. The treatment with dsRNA $(z=-0.578, p=$ $0.563)$ and the interaction between dsRNA treatment and time $(z=1.069, p=0.284)$ were not significantly different from the control.
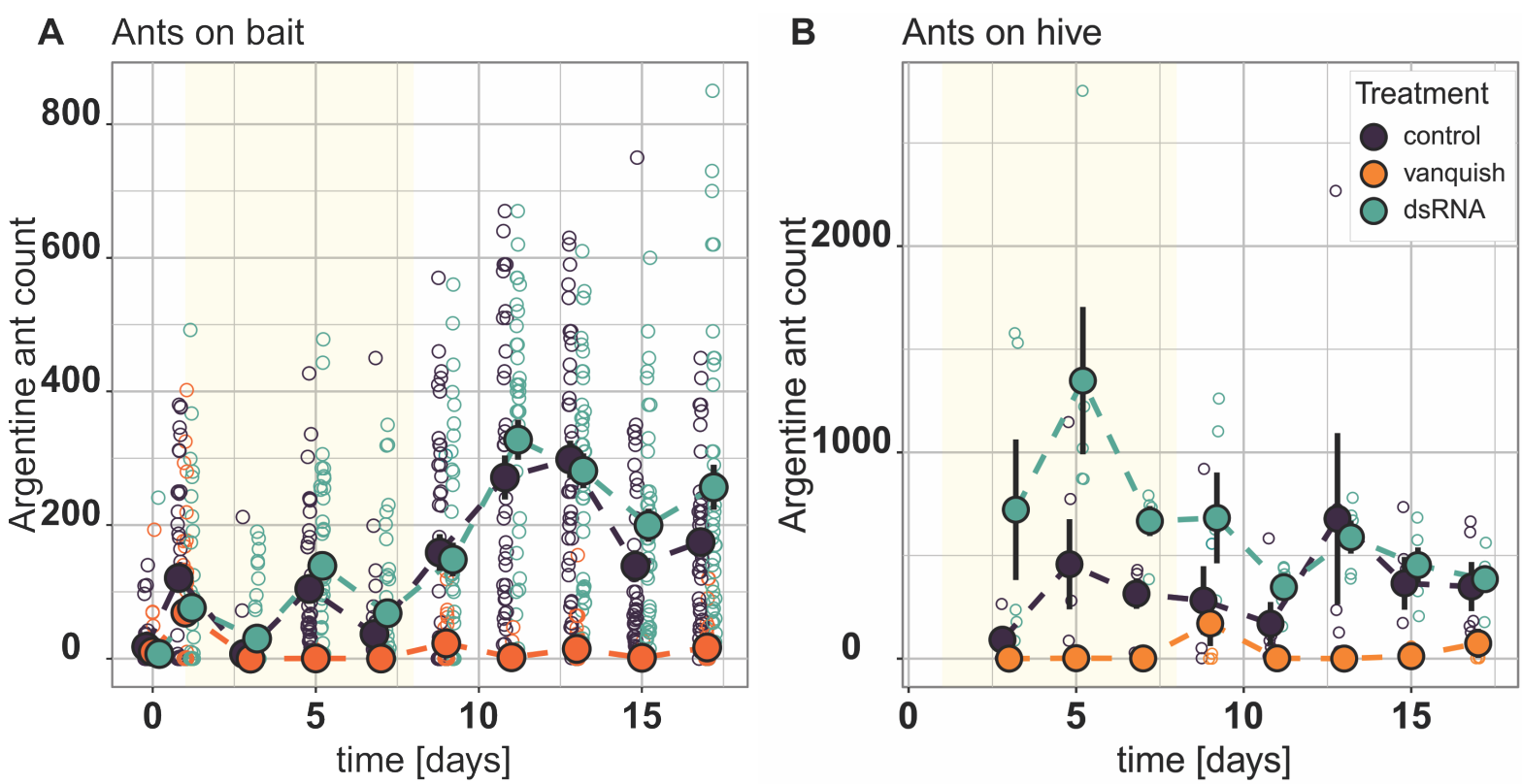

Figure 5.6: Argentine ant abundance on bait dishes and beehives during the experiment. A: Ant numbers were counted from dishes containing a sugar bait after 30 minutes. B: Ant numbers were counted from photos. Ants were treated with a fipronil-based insecticide (Vanquish ${ }^{\circledR}$, orange), double-stranded RNA (dsRNA) targeting dicer-1 and spätzle (green) or a sugar control (purple) over seven days (yellow background). Large circles with error bars show the mean \pm standard error and small circles indicate individual data points. 
The final model for ant numbers on beehives included treatment, time and their interaction as fixed effects and hive as a random effect. The number of ants on baits around beehives and the number of bees on hives did not improve the model fit and were therefore not included. As with the ant counts on bait dishes, ants on beehives were negatively affected by vanquish bait $(z=-3.690, p<0.001$, B in Figure 5.6). The dsRNA treatment, however, had a positive effect on ant numbers compared to the control treatment $(z=2.207, p=0.027$, B in Figure 5.6). Ant counts increased over time, however, this effect was only marginally statistically significant $(\mathrm{z}=1.820, p=0.069)$. This increase after hives were moved into field sites could indicate that beehives attracted ants, so that overall more ants were counted on hives the further the experiment progressed. Interactions between time and dsRNA treatment negatively affected ant numbers $(\mathrm{z}=-0.405, p=0.016)$, however, the effect size was smaller than the positive main effects of dsRNA treatment and time. Interactions between time and vanquish treatment had a marginally positive effect $(\mathrm{z}=1.819, p=0.069$, B in Figure 5.6).

\subsubsection{Effects of ant numbers, dsRNA and vanquish treatment on hive survival}

From the 15 beehives in the study seven absconded within the first ten days from the start of the experiment. Four days after being placed in the site the first two hives absconded; on both, we observed ant numbers exceeding 1500 individuals on the outside of the hive on day three (outliers in Figure S5.2). We observed bee queens leaving their hives and whole colonies leaving their brood and food stores behind (B in Figure 5.7). All hives from which bees absconded had high numbers of ants inside and outside of the hives and we observed ants attacking brood on frames abandoned by bees ( $\mathrm{C}$ in Figure 5.7).

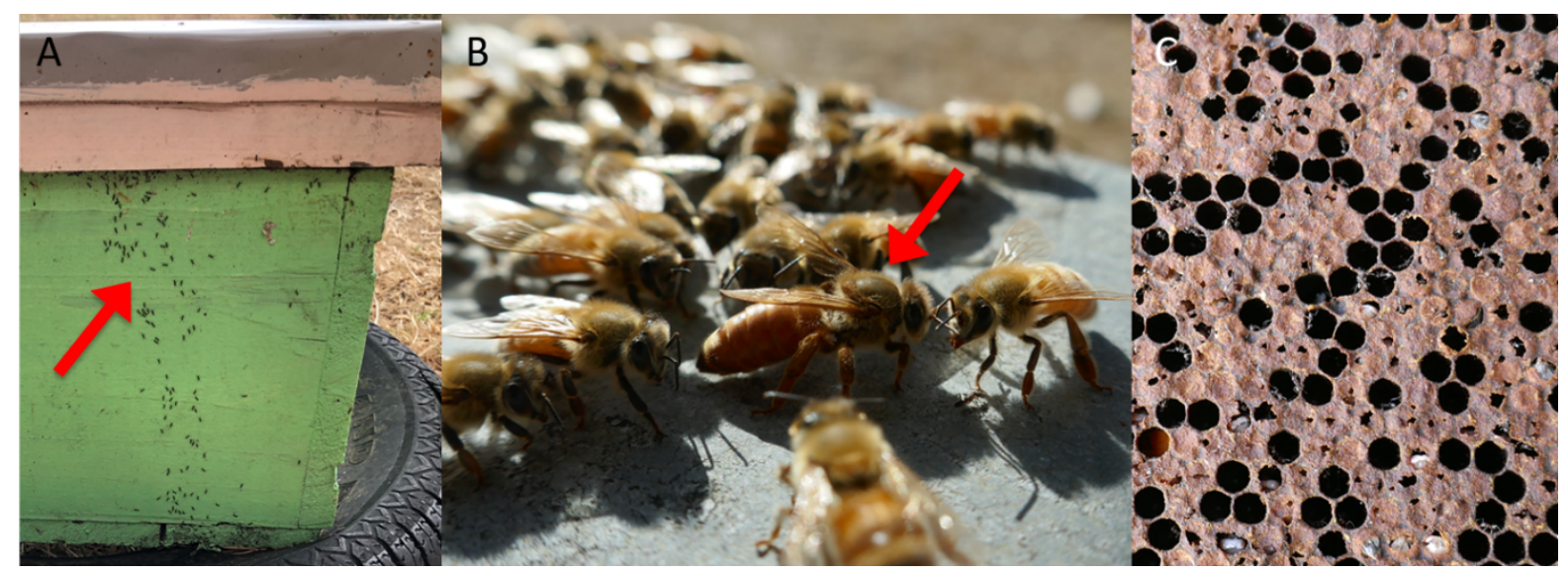

Figure 5.7: Observations of beehives with Argentine ants. A:A trail of Argentine ants on the back of the hive box (red arrow). Ants enter the hive through the gap between the box and the lid. B: A honey bee queen (red arrow) surrounded by workers sitting on top of the hive. The colony abandoned their hive (absconded) due to ant attacks. C: Brood cells inside the hive are uncapped and larvae and pupae are being attacked by ants after bees absconded. Photos by Jana Dobelmann. 
Bee survival in dsRNA and vanquish bait treatment groups ware not different from the sugar control group (both $p=0.134$ ). However, honey bee hive survival in the dsRNA group compared to the vanquish bait group was significantly lower $(p=0.008)$. None of the beehives in the dsRNA treatment and only three out of five hives in the sugar control group survived the 16 days of the experiment. We frequently observed bees hovering over ants that were entering the hive. Although the bees appeared agitated, they did not seem to attack or remove ants from the hive. Photo counts revealed that ants were predominantly entering the hive from the back and to a lesser degree from the left side of the hive (A in Figure 5.7, Figure S5.3). Ants were accessing the hive through gaps between the brood box and lid. Just before bees absconded, ant numbers rapidly increased around the front entrance of the hive (Figure S5.3) and Argentine ants were observed feeding on bee brood when the adult bee populations had left the hive. Approximately three days after the bees absconded, ant numbers started to decrease (Figure S5.3), which probably indicates that ants removed all the food stores and brood within the hive.

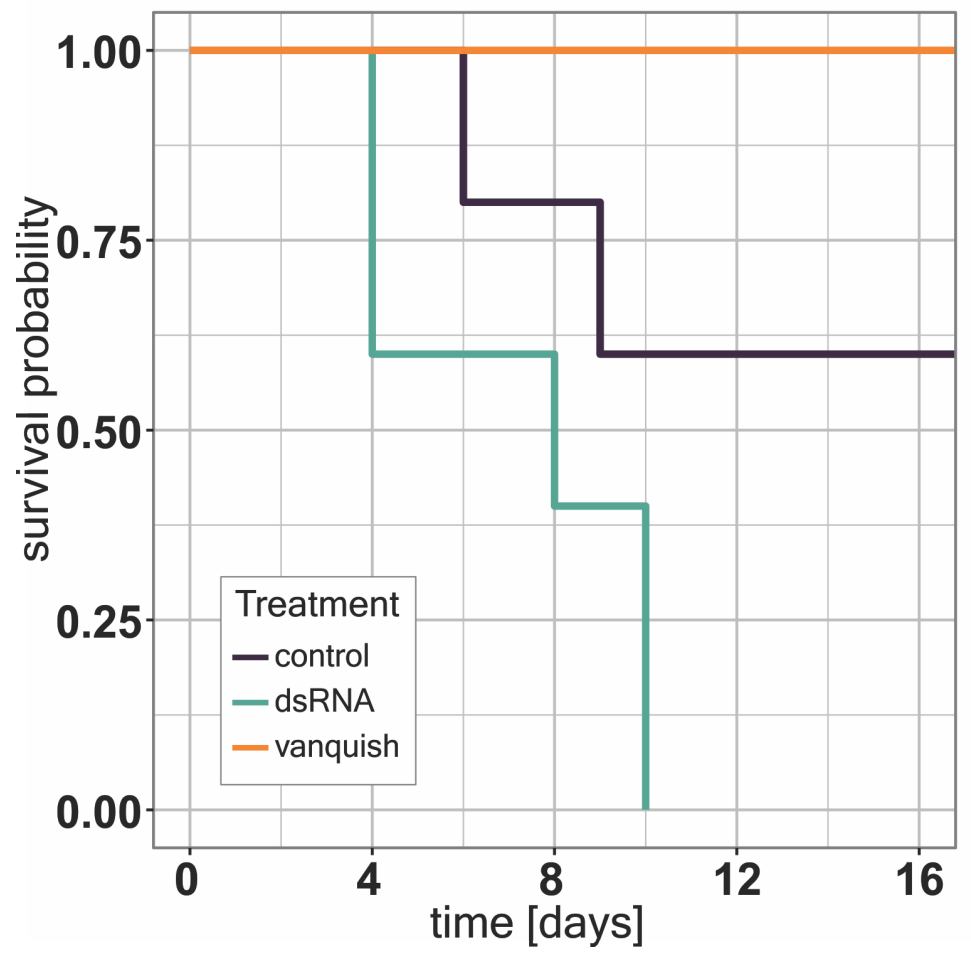

Figure 5.8: Kaplan-Meyer survival curves of 15 beehives after being moved into a field site with Argentine ants and controlling ants using three different treatments. Argentine ants were treated with either Vanquish ${ }^{\circledR}$ bait, a fipronil-based insecticide (orange), sugar water mixed with double-stranded RNA (dsRNA) targeting ant immune genes dicer-1 and spätzle (green) or sugar water only as a control (dark purple). Bee colonies absconded and abandoned their hives when too many ants were entering the hive. Beehives were moved into the site at day zero and treatments were applied from day one until day eight. 


\subsection{Discussion}

In this study, we tested the feasibility and effect of dsRNA as a targeted ant control agent for Argentine ants in apiaries. While we found that conventional ant control significantly reduced ant numbers and improved beehive survival, the dsRNA treatment that we used did not reduce ant numbers and many bee colonies absconded. Expression of targeted genes could not be reduced in the field, yet, we observed some gene expression changes associated with dsRNA application. The current form of delivery, dosage and choice of dsRNA targets failed to achieve a consistent mRNA reduction of the dsRNA target genes and was unable to disrupt ant immunity consistently or affect pathogen levels. Below we discuss factors that could have affected the outcome and further avenues to improve species-specific ant control.

Opposed to laboratory experiments where dicer-1 and spätzle silencing could be achieved for seven and 28 days, respectively (Felden et al. in preparation), we found that, in the field, mRNA levels of the two targeted immune genes were not significantly reduced by dsRNA treatment either during or after the treatment. The pooled sample we used for gene expression analyses may have included ants that fed on the dsRNA bait but also ants that did not. Double-stranded RNA can be introduced into the ant colonies 'social stomach' through foragers feeding on the bait (Hölldobler and Wilson 1990) and therefore may reach individuals that did not actively forage on the bait through trophallaxis. Through this process, dsRNA may be diluted and reach different colony members at a range of dsRNA concentrations, some of which may be too low to induce RNAi. With the goal to reduce immune functions in some ants, we expected immunosuppressed colony members to develop high viral titres and spread viruses to other colony members, thereby achieving population control with potentially longlasting effects. Pathogen levels in ants were not affected by dsRNA treatment. However, we found a significant increase in LhuTLV1 on day five and a consistent yet non-significant trend of higher LhuPCV2 levels in the dsRNA group. Overall, there was no significant viral or bacterial increase, so a pathogen epidemic in the tested nests was unlikely. Lab experiments had found decreases in LhuBLV1 associated with both dsRNA dicer-1 and spätzle, but no increase in any of the pathogens tested (Felden et al. in preparation).

We found that pathogen and gene expression varied significantly over time, which could be caused by large variation within ant nests, differences in sugar feeding or external effects such as environmental conditions. These factors may affect day-to-day gene expression more than dsRNA feeding. Although treatment alone did not affect pathogen loads or gene 
expression, the significant interaction between sampling day and treatment in some gene groups indicates that treatment did affect mRNA levels, but that this effect depends on the day and may only be observed during active treatment (day five and eight) and not beyond ongoing treatment. Yet, the affected groups of immune genes did not include the two immune pathways related to dsRNA targets, i.e. RNAi and Toll, but pathogen recognition, antimicrobial peptides and the Imd/JNK pathway. In lab trials, dicer-1 dsRNA treatment was associated with wideranging reduced expression in immune pathways (Felden et al. in preparation), and although some immune genes showed reduced expression in this study, the reduction did not last over multiple sampling days. Feeding of dsRNA and RNAi can lead to off-target effects that may have affected our results.

The immune genes we targeted in this experiment, dicer-1 and spätzle, were identified as homologues to insect immune-related genes but may also be involved in other processes in the ant host. The two-fold function of RNAi in gene regulation and antiviral immunity could mean that dicer-1 suppression affects immune defences, but that simultaneous changes in gene regulation make it harder to detect these effects. Micro RNAs serve essential functions in biological processes; when dicer-1 silencing inhibits miRNA processing, this can result, for instance, in developmental defects (Lee et al. 2004). Studies have shown that silencing of core genes in the siRNA pathway, dicer-2 and argonaute-2, increases susceptibility to +ssRNA and dsRNA viruses in Drosophila (van Rij et al. 2006, Zambon et al. 2006) without impairing development (van Rij et al. 2006, Wang et al. 2006). A dicer-2 homologue has not yet been identified from the Argentine ant draft genome, although another dicer gene (LOC105670277) was found. Genome sequencing has so far identified two dicer copies in all hymenopterans (Dowling et al. 2016), suggesting that dicer functions across this order are conserved and dicer-1 and dicer in Argentine ants likely correspond to dicer-1 and dicer-2. Whether targeting the other dicer gene instead of dicer-1 could disrupt the siRNA pathway and increase susceptibility to viral pathogens needs to be studied.

Instead of suppressing RNAi genes, other laboratory studies have induced overexpression of RNAi genes, which has shown improved RNAi efficiency. Over-expression of dicer-2 and argonaute- 2 improves RNAi in lepidopterans and dipterans, likely by improving the spread of the RNAi signal (Dietzl et al. 2007, You et al. 2020). Therefore, reducing dicer (or dicer-1) function in ants through RNAi may be counterproductive if RNAi efficiency is reduced. It is possible to achieve an increased efficiency of the experimental RNAi-mediated silencing of genes with a positive feedback loop (Amdam and Omholt 2003, Marco Antonio et 
al. 2008). An example of a positive feedback loop in honey bees is the suppression of Vitellogenin. Once Vitellogenin protein stocks are depleted in silenced bees, production of Vitellogenin is (possibly irreversibly) inhibited through a feedback loop (Marco Antonio et al. 2008).

Apart from the sequence-specific silencing induced by dsRNA, dsRNA itself can act as a pathogen associate molecular pattern (PAMP) through its similarity to virus-derived dsRNA (Flenniken and Andino 2013, Nunes et al. 2013, Yang et al. 2020). Therefore, dsRNA may trigger immune responses or other gene expression changes within the host. For instance, injection of unspecific dsRNA into pea aphids reduces argonaute expression in all RNA pathways and increases dicer-1 expression (Yang et al. 2020), which could indicate activation of the miRNA pathway. The pathogen recognition receptor beta-1,3-glucan binding proteinlike A showed significant upregulation in dsRNA fed ants in the lab (Felden et al. in preparation), suggesting that dsRNA may be recognised as PAMPs. If dsRNA feeding triggers a similar miRNA activation in Argentine ants, dicer- 1 silencing may not be effective, as the host increases expression of this gene in response to the PAMP. In honey bees, the increased RNAi activity through non-specific dsRNA feeding even causes an 'immune boost' that can clear viral infections (Flenniken and Andino 2013). Ants have been shown to share immune responses to boost colony-level immunocompetence through shared feeding (Hamilton et al. 2011), so that recognition of dsRNA as a PAPM could have wide-ranging effects in the nest. To avoid dsRNA triggered 'immune boosts' or other non-specific effects, dsRNA for population control has to be carefully designed.

RNA interference function is even further complicated through viruses interacting with the host responses. As hosts evolve immune responses to combat viral infections, many viruses evolve counter defences ( $\mathrm{Li}$ and Ding 2006). Viral suppressors of RNA silencing (VSRs) have been identified in various invertebrate viruses. These suppressors likely evolved repeatedly and multiple times (Li and Ding 2006, Ding and Voinnet 2007), and include picorna-like viruses that target dicer-2 (van Rij et al. 2006). By targeting RNA pathways to inhibit RNA silencing VSRs improve virus fitness and could drive the evolution of RNA pathway genes. Studies that compared different Drosophila species have found that the siRNA pathway that is primarily involved in antiviral immunity, shows higher evolutionary rates than the miRNA pathway, whose primary function is housekeeping genes (Obbard et al. 2006, Ding and Voinnet 2007). We are only beginning to understand the mechanisms involved in interactions between viruses 
and host immune defences. Yet, VSRs highlight the importance of studying immune responses in natural host populations where viruses are present.

Efficient RNAi can also depend on the dsRNA delivery method. Injecting dsRNA into specific insect tissues, e.g. into the fat body, which is the main site for humoral immune responses, allows us to study immune function and avoids dsRNA degradation in the gut. For insect control in the field, however, dsRNA can only be consumed with uptake through the mid-gut. Uptake from the environment through protein channels into mid-gut cells is mediated by the SID-1 protein (Feinberg and Hunter 2003). This uptake leads to environmental RNAi, which only affects cells that directly take up dsRNA (Huvenne and Smagghe 2010). To achieve silencing in the insects fat body the RNAi signal needs to spread beyond the midgut causing systemic RNAi (Huvenne and Smagghe 2010). The mechanisms that affect systemic RNAi in insects are largely unknown. Lepidopterans, for instance, show reduced RNAi efficiency, most likely caused by fast dsRNA degradation in the gut (Shukla et al. 2016). Protecting dsRNA from degradation inside the 'social stomach' of ant colonies may be important to achieve efficient RNAi. Protection could come from liposomes or dendrimer coated carbon nanotubes, for instance, and may reduce degradation and improve uptake (Edwards et al. 2020, Felden et al. in preparation).

Overall, the dsRNA treatment within our experimental design was ineffective as pest control in an apiary. However, increasing the understanding of RNAi and immune function in ants is key to improving genetic-based pest control methods. Alternative choices of dsRNA targets and sequences or alterations to delivery methods could increase immune silencing for ant control in the field. Understanding RNAi function in ants will inform dsRNA bait design in the future. Critically, combining RNAi and viruses depends on the presence of pathogenic viruses in introduced ant populations to achieve desired ant control (Felden et al. 2019). Sequencing of small RNAs, for instance, could be used to study the processing of dsRNA and responses to viral pathogens within Argentine ants and establish whether dsRNA uptake and dicer-mediated processing are functional. Fluorescently labelled dsRNA could allow tracking of the dsRNA within ants and between colony members to establish whether trophallaxis allows for the transfer of dsRNA from foragers to other colony members. 


\section{Chapter 6: General discussion}

Emerging infectious diseases threaten public health, livestock economies and wildlife (Cleaveland et al. 2001, Dobson and Foufopoulos 2001). Wildlife populations can act as reservoirs for pathogens that cause disease in livestock (Daszak et al. 2000). Several pollinator species have experienced population declines, leading to concerns about malnutrition, food security, and economic losses (Biesmeijer et al. 2006, Potts et al. 2010, Hallmann et al. 2017, Sánchez-Bayo and Wyckhuys 2019). There is increasing evidence that suggests that RNA viruses are a key driver in wild pollinator declines and managed honey bee losses (Manley et al. 2015, McMenamin and Flenniken 2018, Grozinger and Flenniken 2019). Several honey bee-associated viruses have been detected in a range of insects (Singh et al. 2010, Evison et al. 2012, Levitt et al. 2013, McMahon et al. 2015). Disease dynamics in these multi-host pathogens appear to be shaped by host communities (Fountain-Jones et al. 2018) so that human-mediated species introductions can alter dynamics which may lead to disease emergence (Daszak et al. 2000). Little is known about how introduced species affect viruses in pollinator communities (Manley et al. 2015). An improved understanding of the viruses circulating within complex multi-host communities is required to mitigate disease threats (Cleaveland et al. 2001).

Argentine ants (Linepithema humile) are successful invaders (Suarez et al. 2001, Roura-Pascual et al. 2004) that can compete with honey bees for floral resources (Buys 1987, Lach 2007, Sidhu and Wilson Rankin 2016) and are a pest in some New Zealand apiaries (Brown 2017, Brown and Robertson 2018). Several bee-infecting viruses have been detected in Argentine ants (Gruber et al. 2017, Payne et al. 2020) suggesting this ant could affect bee disease (Sebastien et al. 2015). Yet, it was unknown whether Argentine ants impact viral infections in bees and contribute to the epidemic of bee viruses. Here, I studied viral infections in Argentine ants and honey bees, viral host ranges, how species interactions affect disease dynamics in bees, and whether viral pathogens can be harnessed for ant control.

The three main goals of this thesis were:

- To examine disease dynamics within pollinator and arthropod communities that include Argentine ants and honey bees.

$\circ$ To study interactions between Argentine ants and honey bees in apiaries and test whether pathogens and hive survival are affected by species interactions.

○ To explore the feasibility of a novel ant control method that could provide a costeffective long-term strategy to control populations and limit damage from ants. 


\subsection{Summary}

Chapter 2 focused on RNA viruses that are found in honey bees, Argentine ants, and a range of associated arthropods. Deformed wing virus (DWV), Kashmir bee virus (KBV) and Moku virus (MKV) were found to replicate in several hymenopteran species, but also in a spider, a cockroach and a cricket species. Phylogenetic analyses for DWV and KBV showed that shared viral ancestry was significantly associated with the geographic origin of the sample and to a lesser extent with the host species, indicating that strains circulate within pollinator communities and associated insects within geographic regions.

Chapters 3 and 4 examined interactions between honey bees and Argentine ants and whether these interactions affect honey bees. The longitudinal study in Chapter 3 compared survival and pathogen infections in beehives with and without Argentine ants over several months. Hives in sites with Argentine ants had significantly higher DWV levels in autumn compared to the control. Yet, hive survival was not affected by ants throughout the duration of this experiment. In Chapter 4, RNA sequencing (RNA-seq) described the RNA virome of beehives with and without ants. Twelve different viruses were found in the hives studied, among those were three plant viruses and an unclassified virus that had not been detected in honey bees previously. Argentine ant presence was associated with significant increases in Sacbrood virus (SBV) and Tomato ringspot virus (ToRSV) levels, although both viruses have not previously been detected in Argentine ants and ToRSV is unlikely to affect bee health. The changes in viral levels when Argentine ants were present could indicate that ants act as a stressor that affects immune responses, which may increase the replication of viruses that are already present within the beehive. Longer observations could show whether the increased viral titres with ant presence affect long-term colony survival.

Lastly, Chapter 5 investigated a novel control strategy that uses RNA interference (RNAi) to silence immune-related genes in Argentine ants. Reducing antiviral immunity in ants in the field through RNAi was expected to increase viral titres and possibly reduce ant numbers in apiaries. However, no consistent gene-silencing could be achieved and although changes in immune pathways were observed, pathogen levels did not increase and ant numbers were not controlled. Many bee colonies abandoned their hive due to ant attacks and only conventional ant control with the insecticide fipronil could sufficiently control ant numbers to improve bee survival. 


\subsection{Synthesis}

\subsubsection{Do Argentine ants vector bee diseases?}

Multi-host RNA viruses are prevalent across pollinator communities and associated arthropods (Chapter 2, Singh et al. 2010, Evison et al. 2012, Levitt et al. 2013, Manley et al. 2019). In this thesis, I found that Argentine ants, honey bees and a range of associated species carried the same viruses and closely related viral variants, indicating that species barriers are not limiting host ranges and that cross-species transmission is prevalent. Instead, viral genetic variation was determined by geographic location. Ants and bees within the same geographic region may share viruses through overlapping foraging areas or interactions within beehives. Understanding the role ants play in the dynamics of bee-associated viruses is essential to control and mitigate pathogen spread. The high number of ants in some apiaries and their attraction to beehives can affect viral dispersal. The findings in this thesis suggest that Argentine ants could act as a mechanical or biological bee virus vector.

Chapter 3 and 4 provide evidence for Argentine ants potentially transmitting DWV and SBV to honey bees, as their presence was associated with viral increases. Whether ants vector these viruses has not been tested yet. High SBV-levels were correlated with high DWV titres, a virus that has repeatedly been detected in Argentine ants (Sebastien et al. 2015, Gruber et al. 2017, Brenton-Rule 2018, Lester et al. 2019). Sebastien et al. (2015) first suggested that Argentine ants could act as a reservoir for DWV. In concordance with their study, I found DWV replication in Argentine ants (Chapter 2) and high DWV titres in some ant nests (Chapter 3 and 5). To deem this ant species a reservoir population, however, DWV has to persist within Argentine ants which could be tested in laboratory experiments. Deformed wing virus can actively infect and persist in Myrmica rubra colonies for up to 13 weeks after feeding on infected honey bee pupae suggesting that ants are a biological DWV host (Schläppi et al. 2019) and potentially vector the virus.

Argentine ants actively vectoring SBV, however, appears less likely. Argentine ants were not tested for SBV, as the virus has not yet been detected in studies investigating the Argentine ant virome (Gruber et al. 2017, Viljakainen et al. 2018, Felden et al. 2019, Lester et al. 2019), and its presence in ants was therefore considered unlikely. Few reports of SBV in non-Apis bees or other insects exist, indicating the virus may be closely associated with honey bees (Gisder and Genersch 2017, McMahon et al. 2018). Ants may still act as a mechanical vector of SBV, yet, as a mechanical vector, SBV detection would be expected in Argentine 
ants. Payne et al. (2020) found SBV in several ant species (but not Argentine ants), however, only when sampling ants in apiary sites and without detecting SBV replication, indicating that ants acquire SBV from bees.

Controlled transmission experiments could be used to study viral dispersal and indicate whether ants transmit viruses to honey bees. The suggested transmission route from bee to ant is foodborne (Schläppi et al. 2019). How ants could transmit viruses to bees, either while being infected or without being actively infected is unknown. Graystock et al. (2015) designed an experiment in which they showed that honey bees and bumble bees can vector pathogens without being actively infected (mechanical vector) by sharing floral resources. A similar design could be used to study whether ants vector viruses to honey bees or other insects. Honey bees could act as a 'pathogen provider' that contaminate pollen pellets or honey with infectious viral particles (Singh et al. 2010). If Argentine ants consume virus-positive bee-products and transfer the virus from contaminated to virus-free hives or parts of the hive, from which honey bees then acquire viral infections, Argentine ants would act as a mechanical vector.

Bee-infecting viruses have repeatedly been detected in species that prey on honey bees, nonetheless, detection methods are often sensitive so that ingested viruses, for instance from contaminated pollen or infected prey items can lead to a positive test. Perhaps even negative viral strands, indicative of active replication, could be produced in honey bees that are prey items of the ant, causing false detection of viral replication (Posada-Florez et al. 2019). Honey bee-associated viruses may be widely distributed in the environment (Figueroa et al. 2019). Yet, studying viral replication can give insights into which hosts are parasitised by the virus. In Chapter 2, I used strand-specific RT-PCR as an indicator of active viral replication. Positivesense single-stranded RNA viruses replicate via a negative-strand intermediate that can be detected through strand-specific RT-PCR (Craggs et al. 2001). Other methods that indicate active infections include quantitative PCR to track titres over time or deep sequencing to detect antiviral immune responses in the host (Chejanovsky et al. 2014, Remnant et al. 2017, Baty et al. 2020). Antiviral immune responses in the insect host involve RNAi pathways that cleave double-stranded RNA into small RNAs through dicer enzymes (Ding 2010, Chejanovsky et al. 2014). The size distribution of small RNAs, the ratio of negative- to positive-sense RNAs, and the genome position of the small RNA reads can thereby be indicative of active or inactive infections and host immune responses (Remnant et al. 2017). Characterising viral small RNA patterns in honey bees and Argentine ants and comparing these could provide information 
about whether ants ingest viruses (and possibly negative viral strands) through preying on infected honey bees or produce immune responses targeting the virus.

\subsubsection{Stressors can trigger viral diseases in honey bees}

The list of stress factors affecting honey bee health is long. It includes Varroa and DWV, other RNA viruses, the microsporidian Nosema ceranae, monotonous diets, pesticides and landscape fragmentation (Evans and Schwarz 2011, Di Prisco et al. 2013, Dolezal et al. 2019, Grozinger and Flenniken 2019). Stressors may facilitate disease emergence (Manley et al. 2015). Deformed wing virus is highly prevalent in honey bees (this thesis, de Miranda and Genersch 2010, Gallagher et al. 2018). The virus commonly persists covertly (Chen and Siede 2007), and the honey bee host may fail to clear infections, possibly because DWV suppresses immune function (Nazzi et al. 2012). Nazzi and Pennacchio (2014) argue that stressors could overwhelm immune responses that are already affected by the virus. Multiple interacting stress factors could affect immune function which may lead to DWV outbreaks (Nazzi and Pennacchio 2018). Argentine ant presence was associated with increases in DWV and transcriptomic changes in immune-related genes (Chapter 3 and 4). I argue that Argentine ants should be considered as a stressor that can cause elevated infection rates (Figure 6.1). Their presence could trigger stress responses that then overwhelm immune function and lead to changes in viral dynamics. Although it is extremely difficult to pinpoint or disentangle the interactions between a specific pathogen and a stressor (Lafferty 2010), particularly in field studies.

Further study of ant-bee interactions can improve the understanding of disease transmission and how bees respond to ant stress. Bees generally show avoidance behaviour when encountering ants on flowers (Sidhu and Wilson Rankin 2016), but when Argentine ants attack beehives, bees hovered over ants and seemed agitated (Chapter 5). Inside the hive, we found Argentine ants preying on emerging workers and bee brood. Disturbance-free observations of ant-bee interactions through observational hives could be used to study bee defensive behaviour and allow insight into ant movements inside the hive that may be key to viral spread. 


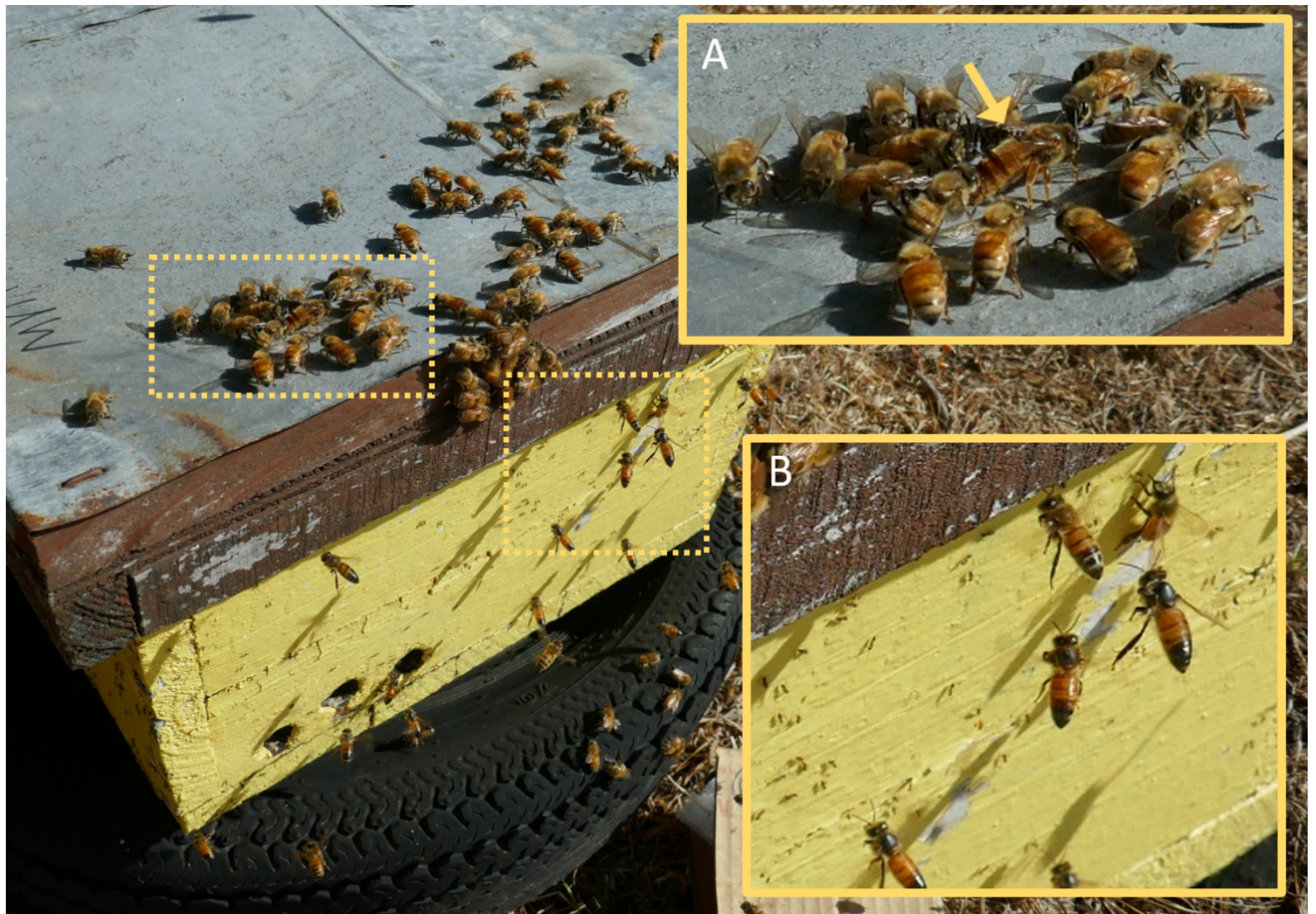

Figure 6.1: Argentine ants cause stress in honey bees through attacks on beehives that may be linked to changes in pathogen infections. The photo shows a bee colony that is being attacked by ants and is abandoning its hive. A: A queen bee (yellow arrow) surrounded by workers is sitting on top of the hive box. B: Bees hovering over ants on the outside of the hive. Photo by Jana Dobelmann.

\subsubsection{Do increased viral loads in association with Argentine ants affect hive} mortality?

Increased DWV levels in autumn are correlated with overwinter collapses (Dainat et al. 2012). Brenton-Rule (2018) found that beehive survival with Argentine ants was reduced to approximately $50 \%$ over 5 months compared to almost $90 \%$ in Argentine ant-free hives. Argentine ant control in apiaries with conventional insecticides improved bee survival by reducing absconding behaviour (Chapter 5) but over the 7 months when honey bees were studied in sites with and without Argentine ants, hive survival did not differ between sites (Chapter 3). Two beehives in sites with Argentine ants in the experiment in Chapter 3 were found empty after only one month. Together with the observations in Chapter 5, it appears likely that these hives absconded shortly after we placed beehives in the sites, a behaviour linked to ant attacks (Payne et al. 2020). The small hives used in this thesis may be more prone to failure, while Brenton-Rule (2018) observed hives that were already established in sites with and without ants and potentially consisted of larger colonies. Yet, the high mortality rates 
observed in Chapter 3 were surprising and indicate that hives were in poor health and other stress factors not assessed in the experiment possibly contributed to mortality. Manipulative field experiments often have low levels of replication coupled with weak effect sizes which can lead to falsely accepting the null hypothesis, i.e. not detecting effects (Lemoine et al. 2016). Increasing sample sizes can reduce this error but financial limitations do not always allow for large field experiments with extensive molecular analysis. Applying more emphasis to effect sizes than statistical significance (Lemoine et al. 2016) can be useful to interpret findings in such cases.

\subsubsection{Argentine ant control relies on pathogenic viruses within ant populations}

Controlling Argentine ants in apiaries could significantly improve bee health. Using RNAi to control ant populations firstly depends on the ability to alter immune gene expression, but also critically depends on the presence of pathogenic viruses within ant populations (Gruber et al. 2017). The use of RNA-seq and other next-generation sequencing tools in a broad range of insects has greatly enriched our understanding of virus biodiversity ( $\mathrm{Li}$ et al. 2015). Future efforts need to focus on the effect viruses have on their insect hosts and identify pathogenic ones. Argentine ants are naturally infected with a range of viruses (Gruber et al. 2017, Viljakainen et al. 2018) but data on virus pathogenicity in Argentine ants is scarce (Baty et al. 2020). Studies in L. humile have primarily focused on virus discovery (Gruber et al. 2017, Viljakainen et al. 2018, Baty et al. 2020). Lower viral diversity in the introduced ranges of Argentine ants could potentially indicate that highly pathogenic viruses are absent from invasive populations (Felden et al. 2019). Negative viral strands indicating active viral replication of DWV and KBV in Argentine ants have been detected (this thesis, Gruber et al. 2017), yet, replication of ant-associated viruses was only assessed for Linepithema humile bunya-like virus 1 (Chapter 2) and has otherwise not been assessed. Future studies need to investigate antiviral immune responses and clinical symptoms of viruses in ants. Sequencing and characterisation of small RNA populations in Argentine ants, for instance, could show whether ants show RNAi responses to viruses and determine which viruses actively infect the host.

Acute bee paralysis virus (ABPV) was the first bee-infecting virus with health effects in an ant species (Schläppi et al. 2020). Schläppi et al. (2020) fed ABPV infected bee pupae to field-caught ant colonies (Lasius spp.) and found clinical effects at the individual- and colonylevel. The virus forms a species complex with Israeli acute paralysis virus (IAPV) and KBV 
(de Miranda et al. 2010). In New Zealand, KBV is the dominating virus out of the complex (de Miranda et al. 2010) and IAPV is completely absent (McFadden et al. 2015). Whether KBV shows clinical effects in ants is unknown but the high prevalence in Argentine ants (Chapter 2) and observations of KBV-positive ants in apiaries without KBV-positive bees (Chapter 3) indicate that the virus may persist in ant nests without ongoing transmission from honey bees to ants. Nevertheless, inferring the direction of transmission based on viral prevalence and titre is challenging as these are known to vary temporally (Runckel et al. 2011).

\subsection{Conclusion and outlook}

Pathogen communities are shaped by within- and between-species interactions, which can change with species introductions (Daszak et al. 2000, Fountain-Jones et al. 2018). The ongoing introduction of alien species, including the pathogens they may harbour, in combination with environmental stressors will further affect pollinator health (Potts et al. 2010). Invasive ant species can impact ecosystem functioning (Holway et al. 2002). With the difficulties of controlling ant populations (e.g. Hoffmann et al. 2016) and future climates that may promote range expansions (Hartley and Lester 2003, Roura-Pascual et al. 2004, RouraPascual et al. 2011), pest ants in apiaries may become more frequent. Here, I showed that invasive Argentine ants are associated with increases in viral titres in honey bees. The mechanisms by which ants affect bee disease are unknown, although there is some evidence for ants vectoring pathogens or causing stress responses that affect bee immunity. This novel contribution to the field of honey bee-associated viruses further highlights the risk of disrupting pollination through invasive species. Ants or any other stressor affecting viral disease in honey bees could cause viral spillover to wild bee species and associates (Fürst et al. 2014, Alger et al. 2019, Manley et al. 2019) that can further affect pollination and food security (Goulson et al. 2015). New and environmentally-friendly methods to control invasive species are urgently needed to reduce the risk of disrupting pollination services. 


\section{References.}

Adler, L. S., K. M. Michaud, S. P. Ellner, S. H. McArt, P. C. Stevenson, and R. E. Irwin. 2018. Disease where you dine: Plant species and floral traits associated with pathogen transmission in bumble bees. Ecology 99:2535-2545.

Alger, S. A., P. A. Burnham, H. F. Boncristiani, and A. K. Brody. 2019. RNA virus spillover from managed honeybees (Apis mellifera) to wild bumblebees (Bombus spp.). PLoS One 14:e0217822.

Alizon, S., J. C. de Roode, and Y. Michalakis. 2013. Multiple infections and the evolution of virulence. Ecology Letters 16:556-567.

Altschul, S. F., W. Gish, W. Miller, E. W. Myers, and D. J. Lipman. 1990. Basic local alignment search tool. Journal of Molecular Biology 215:403-410.

Amdam, G. V., and S. W. Omholt. 2003. The hive bee to forager transition in honeybee colonies: The double repressor hypothesis. Journal of Theoretical Biology 223:451464.

Andersen, C. L., J. L. Jensen, and T. F. Ørntoft. 2004. Normalization of real-time quantitative reverse transcription-PCR data: A model-based variance estimation approach to identify genes suited for normalization, applied to bladder and colon cancer data sets. Cancer Research 64:5245-5250.

Arbetman, M. P., I. Meeus, C. L. Morales, M. A. Aizen, and G. Smagghe. 2013. Alien parasite hitchhikes to Patagonia on invasive bumblebee. Biological Invasions 15:489-494.

Archie, E. A., G. Luikart, and V. O. Ezenwa. 2009. Infecting epidemiology with genetics: A new frontier in disease ecology. Trends in Ecology \& Evolution 24:21-30.

Bailes, E. J., J. Bagi, J. Coltman, M. T. Fountain, L. Wilfert, and M. J. F. Brown. 2020. Host density drives viral, but not trypanosome, transmission in a key pollinator. Proceedings of the Royal Society B: Biological Sciences 287:20191969.

Bailes, E. J., K. R. Deutsch, J. Bagi, L. Rondissone, M. J. F. Brown, and O. T. Lewis. 2018. First detection of bee viruses in hoverfly (syrphid) pollinators. Biology Letters 14:20180001.

Bailey, L. 1967. The incidence of virus diseases in the honey bee. Annals of Applied Biology 60:43-48.

Bailey, L. 1968. Honey bee pathology. Annual Review of Entomology 13:191-212. 
Barribeau, S. M., B. M. Sadd, L. du Plessis, and P. Schmid-Hempel. 2014. Gene expression differences underlying genotype-by-genotype specificity in a host-parasite system. Proceedings of the National Academy of Sciences 111:3496-3501.

Baty, J. W., M. Bulgarella, J. Dobelmann, A. Felden, and P. J. Lester. 2020. Viruses and their effects in ants (Hymenoptera: Formicidae). Myrmecological News 30:213-228.

Baum, J. A., T. Bogaert, W. Clinton, G. R. Heck, P. Feldmann, O. Ilagan, S. Johnson, G. Plaetinck, T. Munyikwa, M. Pleau, T. Vaughn, and J. Roberts. 2007. Control of coleopteran insect pests through RNA interference. Nature Biotechnology 25:13221326.

Beaurepaire, A., N. Piot, V. Doublet, K. Antunez, E. Campbell, P. Chantawannakul, N. Chejanovsky, A. Gajda, M. Heerman, and D. Panziera. 2020. Diversity and global distribution of viruses of the western honey bee, Apis mellifera. Insects 11:239.

Benaets, K., A. V. Geystelen, D. Cardoen, L. D. Smet, D. C. d. Graaf, L. Schoofs, M. H. D. Larmuseau, L. E. Brettell, S. J. Martin, and T. Wenseleers. 2017. Covert deformed wing virus infections have long-term deleterious effects on honeybee foraging and survival. Proceedings of the Royal Society B: Biological Sciences 284:20162149.

Benjamini, Y., and Y. Hochberg. 1995. Controlling the False Discovery Rate-a practical and powerful approach to multiple testing. Journal of the Royal Statistical Society Series B-Statistical Methodology 57:289-300.

Benjeddou, M., N. Leat, M. Allsopp, and S. Davison. 2001. Detection of acute bee paralysis virus and black queen cell virus from honeybees by reverse transcriptase PCR. Applied and Environmental Microbiology 67:2384-2387.

Bertelsmeier, C., S. Ollier, A. Liebhold, and L. Keller. 2017. Recent human history governs global ant invasion dynamics. Nature Ecology \& Evolution 1:0184.

Biesmeijer, J. C., S. P. M. Roberts, M. Reemer, R. Ohlemüller, M. Edwards, T. Peeters, A. P. Schaffers, S. G. Potts, R. Kleukers, C. D. Thomas, J. Settele, and W. E. Kunin. 2006. Parallel declines in pollinators and insect-pollinated plants in Britain and the Netherlands. Science 313:351-354.

Bingsohn, L., E. Knorr, A. Billion, K. E. Narva, and A. Vilcinskas. 2017. Knockdown of genes in the Toll pathway reveals new lethal RNA interference targets for insect pest control. Insect Molecular Biology 26:92-102.

Blacquiere, T., G. Smagghe, C. A. van Gestel, and V. Mommaerts. 2012. Neonicotinoids in bees: A review on concentrations, side-effects and risk assessment. Ecotoxicology 21:973-992.

Boecking, O., and M. Spivak. 1999. Behavioral defenses of honey bees against Varroa jacobsoni Oud. Apidologie 30:141-158. 
Boncristiani, H. F., G. Di Prisco, J. S. Pettis, M. Hamilton, and Y. P. Chen. 2009. Molecular approaches to the analysis of deformed wing virus replication and pathogenesis in the honey bee, Apis mellifera. Virology Journal 6:221.

Bonning, B. C., and W. A. Miller. 2010. Dicistroviruses. Annual Review of Entomology 55:129-150.

Bouckaert, R., J. Heled, D. Kühnert, T. Vaughan, C.-H. Wu, D. Xie, M. A. Suchard, A. Rambaut, and A. J. Drummond. 2014. BEAST 2: A software platform for bayesian evolutionary analysis. PLoS Computational Biology 10:e1003537.

Bowen-Walker, P. L., S. J. Martin, and A. Gunn. 1999. The transmission of deformed wing virus between honeybees (Apis mellifera L.) by the ectoparasitic mite Varroa jacobsoni Oud. Journal of Invertebrate Pathology 73:101-106.

Brenton-Rule, E. C. 2018. The interface between invasive species science and legal regulation, using Hymenopteran species and their pathogens as a model system. PhD Thesis. Victoria University of Wellington, Wellington, New Zealand.

Brettell, L. E., D. C. Schroeder, and S. J. Martin. 2019. RNAseq analysis reveals virus diversity within Hawaiian apiary insect communities. Viruses 11:397.

Brodschneider, R., A. Gray, N. Adjlane, A. Ballis, V. Brusbardis, J. D. Charriere, R. Chlebo, M. F. Coffey, B. Dahle, D. C. de Graaf, M. M. Drazic, G. Evans, M. Fedoriak, I. Forsythe, A. Gregorc, U. Grzeda, A. Hetzroni, L. Kauko, P. Kristiansen, M. Martikkala, R. Martin-Hernandez, C. A. Medina-Flores, F. Mutinelli, A. Raudmets, V. A. Ryzhikov, N. Simon-Delso, J. Stevanovic, A. Uzunov, F. Vejsnaes, S. Wohl, M. Zammit-Mangion, and J. Danihlik. 2018. Multi-country loss rates of honey bee colonies during winter 2016/2017 from the COLOSS survey. Journal of Apicultural Research 57:452-457.

Brown, P. 2017. New Zealand colony loss survey report - 2016. Ministry of Primary Industries, Wellington, New Zealand.

Brown, P., L. E. Newstrom-Lloyd, B. J. Foster, P. H. Badger, and J. A. McLean. 2018. Winter 2016 honey bee colony losses in New Zealand. Journal of Apicultural Research 57:278291.

Brown, P., and T. Robertson. 2018. Report on the 2017 New Zealand Colony Loss Survey. Ministry for Primary Industries, New Zealand.

Brutscher, L. M., K. F. Daughenbaugh, and M. L. Flenniken. 2015. Antiviral defense mechanisms in honey bees. Current Opinion in Insect Science 10:71-82.

Brutscher, L. M., K. F. Daughenbaugh, and M. L. Flenniken. 2017. Virus and dsRNA-triggered transcriptional responses reveal key components of honey bee antiviral defense. Scientific Reports 7:6448. 
Buczkowski, G., and T. C. Wossler. 2019. Controlling invasive Argentine ants, Linepithema humile, in conservation areas using horizontal insecticide transfer. Scientific Reports 9:19495.

Burand, J. P., and W. B. Hunter. 2013. RNAi: Future in insect management. Journal of Invertebrate Pathology 112 Suppl: S68-74.

Buys, B. 1987. Competition for nectar between Argentine ants (Iridomyrmex humilis) and honeybees (Apis mellifera) on black ironbark (Eucalyptus sideroxylon). South African Journal of Zoology 22:173-174.

Buys, B. 1990. Relationships between Argentine ants and honeybees in South Africa. Pages 519-524 in R. K. Vander Meer, K. Jaffe, and A. Cedeno, editors. Applied Myrmecology: A World Perspective. CRC Press.

Calisher, C. H., J. E. Childs, H. E. Field, K. V. Holmes, and T. Schountz. 2006. Bats: Important reservoir hosts of emerging viruses. Clinical Microbiology Reviews 19:531-545.

Capinha, C., F. Essl, H. Seebens, D. Moser, and H. M. Pereira. 2015. The dispersal of alien species redefines biogeography in the Anthropocene. Science 348:1248-1251.

Celle, O., P. Blanchard, V. Olivier, F. Schurr, N. Cougoule, J. P. Faucon, and M. Ribiere. 2008. Detection of chronic bee paralysis virus (CBPV) genome and its replicative RNA form in various hosts and possible ways of spread. Virus Research 133:280-284.

Chantawannakul, P., L. Ward, N. Boonham, and M. Brown. 2006. A scientific note on the detection of honeybee viruses using real-time PCR (TaqMan) in Varroa mites collected from a Thai honeybee (Apis mellifera) apiary. Journal of Invertebrate Pathology 91:6973.

Charles, J., D. Suckling, D. Allan, K. Froud, P. Dentener, P. Connolly, and H. Verberne. 2002. The distribution of Argentine ants in New Zealand: Can a ten-year old decision not to eradicate be re-visited? Pages 109-124 in S. L. Goldsson and D. M. Suckling, editors. Defending the Green Oasis: New Zealand Biosecurity and Science, Lincoln: New Zealand Plant Protection Society Inc.

Chejanovsky, N., R. Ophir, M. S. Schwager, Y. Slabezki, S. Grossman, and D. Cox-Foster. 2014. Characterization of viral siRNA populations in honey bee colony collapse disorder. Virology 454-455:176-183.

Chen, Y., J. D. Evans, I. B. Smith, and J. S. Pettis. 2008. Nosema ceranae is a long-present and wide-spread microsporidian infection of the European honey bee (Apis mellifera) in the United States. Journal of Invertebrate Pathology 97:186-188.

Chen, Y., M. G. Redinbaugh, and A. P. Michel. 2015. Molecular interactions and immune responses between Maize fine streak virus and the leafhopper vector Graminella 
nigrifrons through differential expression and RNA interference. Insect Molecular Biology 24:391-401.

Chen, Y., Y. Zhao, J. Hammond, H. T. Hsu, J. Evans, and M. Feldlaufer. 2004. Multiple virus infections in the honey bee and genome divergence of honey bee viruses. Journal of Invertebrate Pathology 87:84-93.

Chen, Y. P., J. S. Pettis, M. Corona, W. P. Chen, C. J. Li, M. Spivak, P. K. Visscher, G. DeGrandi-Hoffman, H. Boncristiani, Y. Zhao, D. vanEngelsdorp, K. Delaplane, L. Solter, F. Drummond, M. Kramer, W. I. Lipkin, G. Palacios, M. C. Hamilton, B. Smith, S. K. Huang, H. Q. Zheng, J. L. Li, X. Zhang, A. F. Zhou, L. Y. Wu, J. Z. Zhou, M.-L. Lee, E. W. Teixeira, Z. G. Li, and J. D. Evans. 2014. Israeli acute paralysis virus: Epidemiology, pathogenesis and implications for honey bee health. PLoS Pathogens 10:e1004261.

Chen, Y. P., and R. Siede. 2007. Honey bee viruses. Advances in Virus Research 70:33-80.

Cleaveland, S., M. Laurenson, and L. Taylor. 2001. Diseases of humans and their domestic mammals: Pathogen characteristics, host range and the risk of emergence. Philosophical Transactions of the Royal Society of London. Series B: Biological Sciences 356:991-999.

Cooling, M., S. Hartley, D. A. Sim, and P. J. Lester. 2012. The widespread collapse of an invasive species: Argentine ants (Linepithema humile) in New Zealand. Biology Letters 8:430-433.

Cooling, M., D. A. Sim, and P. J. Lester. 2015. Density-dependent effects of an invasive ant on a ground-dwelling arthropod community. Environmental Entomology 44:44-53.

Corin, S. E., K. L. Abbott, P. A. Ritchie, and P. J. Lester. 2007. Large scale unicoloniality: The population and colony structure of the invasive Argentine ant (Linepithema humile) in New Zealand. Insectes Sociaux 54:275-282.

Cornman, R. S. 2019. Relative abundance and molecular evolution of lake Sinai virus (Sinaivirus) clades. PeerJ 7:e6305.

Cornman, R. S., D. R. Tarpy, Y. Chen, L. Jeffreys, D. Lopez, J. S. Pettis, D. vanEngelsdorp, and J. D. Evans. 2012. Pathogen webs in collapsing honey bee colonies. PLoS One 7:e43562.

Cox-Foster, D. L., S. Conlan, E. C. Holmes, G. Palacios, J. D. Evans, N. A. Moran, P.-L. Quan, T. Briese, M. Hornig, D. M. Geiser, V. Martinson, D. vanEngelsdorp, A. L. Kalkstein, A. Drysdale, J. Hui, J. Zhai, L. Cui, S. K. Hutchison, J. F. Simons, M. Egholm, J. S. Pettis, and W. I. Lipkin. 2007. A Metagenomic Survey of microbes in honey bee colony collapse disorder. Science 318:283-287. 
Craggs, J. K., J. K. Ball, B. J. Thomson, W. L. Irving, and A. M. Grabowska. 2001. Development of a strand-specific RT-PCR based assay to detect the replicative form of hepatitis C virus RNA. Journal of Virological Methods 94:111-120.

Cremer, S. 2019. Pathogens and disease defense of invasive ants. Current Opinion in Insect Science 33:63-68.

Cremer, S., and M. Sixt. 2009. Analogies in the evolution of individual and social immunity. Philosophical Transactions of the Royal Society B: Biological Sciences 364:129-142.

Dainat, B., J. D. Evans, Y. P. Chen, L. Gauthier, and P. Neumann. 2012. Predictive markers of honey bee colony collapse. PLoS One 7.

Darzi, E., E. Smith, D. Shargil, O. Lachman, L. Ganot, and A. Dombrovsky. 2018. The honeybee Apis mellifera contributes to Cucumber green mottle mosaic virus spread via pollination. Plant Pathology 67:244-251.

Daszak, P., A. A. Cunningham, and A. D. Hyatt. 2000. Emerging infectious diseases of wildlife - threats to biodiversity and human health. Science 287:443-449.

Daszak, P., A. A. Cunningham, and A. D. Hyatt. 2001. Anthropogenic environmental change and the emergence of infectious diseases in wildlife. Acta Tropica 78:103-116.

Daszak, P., A. A. Cunningham, and A. D. Hyatt. 2003. Infectious disease and amphibian population declines. Diversity and Distributions 9:141-150.

de Miranda, J. R., L. Bailey, B. V. Ball, P. Blanchard, G. E. Budge, N. Chejanovsky, Y.-P. Chen, L. Gauthier, E. Genersch, D. C. de Graaf, M. Ribière, E. Ryabov, L. De Smet, and J. J. M. van der Steen. 2015. Standard methods for virus research in Apis mellifera. Journal of Apicultural Research 52:1-56.

de Miranda, J. R., G. Cordoni, and G. Budge. 2010. The Acute bee paralysis virus-Kashmir bee virus-Israeli acute paralysis virus complex. Journal of Invertebrate Pathology $\mathbf{1 0 3}$ Suppl 1:S30-47.

de Miranda, J. R., and E. Genersch. 2010. Deformed wing virus. Journal of Invertebrate Pathology 103 Suppl 1:S48-61.

DeGrandi-Hoffman, G., Y. Chen, E. Huang, and M. H. Huang. 2010. The effect of diet on protein concentration, hypopharyngeal gland development and virus load in worker honey bees (Apis mellifera L.). Journal of Insect Physiology 56:1184-1191.

Di Prisco, G., D. Annoscia, M. Margiotta, R. Ferrara, P. Varricchio, V. Zanni, E. Caprio, F. Nazzi, and F. Pennacchio. 2016. A mutualistic symbiosis between a parasitic mite and a pathogenic virus undermines honey bee immunity and health. Proceedings of the National Academy of Sciences 113:3203-3208. 
Di Prisco, G., V. Cavaliere, D. Annoscia, P. Varricchio, E. Caprio, F. Nazzi, G. Gargiulo, and F. Pennacchio. 2013. Neonicotinoid clothianidin adversely affects insect immunity and promotes replication of a viral pathogen in honey bees. Proceedings of the National Academy of Sciences 110:18466-18471.

Dietzl, G., D. Chen, F. Schnorrer, K.-C. Su, Y. Barinova, M. Fellner, B. Gasser, K. Kinsey, S. Oppel, S. Scheiblauer, A. Couto, V. Marra, K. Keleman, and B. J. Dickson. 2007. A genome-wide transgenic RNAi library for conditional gene inactivation in Drosophila. Nature 448:151-156.

Ding, S.-W., and O. Voinnet. 2007. Antiviral immunity directed by small RNAs. Cell 130:413426.

Ding, S. W. 2010. RNA-based antiviral immunity. Nature Reviews Immunology 10:632-644.

Dobelmann, J., A. Felden, and P. J. Lester. 2020. Genetic strain diversity of multi-host RNA viruses that infect a wide range of pollinators and associates is shaped by geographic origins. Viruses 12:358.

Dobson, A. 2004. Population dynamics of pathogens with multiple host species. The American Naturalist 164:64-78.

Dobson, A., and J. Foufopoulos. 2001. Emerging infectious pathogens of wildlife. Philosophical Transactions of the Royal Society of London. Series B: Biological Sciences 356:1001-1012.

Dolezal, A. G., J. Carrillo-Tripp, T. M. Judd, W. Allen Miller, B. C. Bonning, and A. L. Toth. 2019. Interacting stressors matter: Diet quality and virus infection in honeybee health. Royal Society Open Science 6:181803.

Dolezal, A. G., S. D. Hendrix, N. A. Scavo, J. Carrillo-Tripp, M. A. Harris, M. J. Wheelock, M. E. O’Neal, and A. L. Toth. 2016. Honey bee viruses in wild bees: Viral prevalence, loads, and experimental inoculation. PLoS One 11:e0166190.

Domingo, E., and J. J. Holland. 1997. RNA virus mutations and fitness for survival. Annual Review Microbiology 51:151-178.

Donovan, B. J. 2007. Apoidea (Insecta: Hymenoptera). Page 295 in T. K. Crosby, editor. Fauna of New Zealand, Landcare Research Ltd, Christchurch, New Zealand.

Doublet, V., Y. Poeschl, A. Gogol-Doring, C. Alaux, D. Annoscia, C. Aurori, S. M. Barribeau, O. C. Bedoya-Reina, M. J. Brown, J. C. Bull, M. L. Flenniken, D. A. Galbraith, E. Genersch, S. Gisder, I. Grosse, H. L. Holt, D. Hultmark, H. M. Lattorff, Y. Le Conte, F. Manfredini, D. P. McMahon, R. F. Moritz, F. Nazzi, E. L. Nino, K. Nowick, R. P. van Rij, R. J. Paxton, and C. M. Grozinger. 2017. Unity in defence: Honeybee workers exhibit conserved molecular responses to diverse pathogens. BMC Genomics 18:207. 
Dowling, D., T. Pauli, A. Donath, K. Meusemann, L. Podsiadlowski, M. Petersen, R. S. Peters, C. Mayer, S. Liu, X. Zhou, B. Misof, and O. Niehuis. 2016. Phylogenetic origin and diversification of RNAi pathway genes in insects. Genome Biology and Evolution 8:3784-3793.

Drake, J. W., and J. J. Holland. 1999. Mutation rates among RNA viruses. Proceedings of the National Academy of Sciences 96:13910-13913.

Drummond, A. J., O. G. Pybus, A. Rambaut, R. Forsberg, and A. G. Rodrigo. 2003. Measurably evolving populations. Trends in Ecology \& Evolution 18:481-488.

Edosa, T. T., Y. H. Jo, M. Keshavarz, Y. M. Bae, D. H. Kim, Y. S. Lee, and Y. S. Han. 2020. TmSpz6 is essential for regulating the immune response to Escherichia coli and Staphylococcus aureus infection in Tenebrio molitor. Insects 11:105.

Edwards, C. H., C. R. Christie, A. Masotti, A. Celluzzi, A. Caporali, and E. M. Campbell. 2020. Dendrimer-coated carbon nanotubes deliver dsRNA and increase the efficacy of gene knockdown in the red flour beetle Tribolium castaneum. Scientific Reports 10:12422.

Ellner, S. P., W. H. Ng, and C. R. Myers. 2020. Individual specialization and multihost epidemics: Disease spread in plant-pollinator networks. The American Naturalist 195:E118-E131.

Evans, J. D., and R. S. Schwarz. 2011. Bees brought to their knees: Microbes affecting honey bee health. Trends in Microbiology 19:614-620.

Evans, J. D., R. S. Schwarz, Y. P. Chen, G. Budge, R. S. Cornman, P. De la Rua, J. R. de Miranda, S. Foret, L. Foster, L. Gauthier, E. Genersch, S. Gisder, A. Jarosch, R. Kucharski, D. Lopez, C. M. Lun, R. F. A. Moritz, R. Maleszka, I. Muñoz, and M. A. Pinto. 2013. Standard methods for molecular research in Apis mellifera. Journal of Apicultural Research 52:1-54.

Evison, S. E., K. E. Roberts, L. Laurenson, S. Pietravalle, J. Hui, J. C. Biesmeijer, J. E. Smith, G. Budge, and W. O. Hughes. 2012. Pervasiveness of parasites in pollinators. PLoS One 7:e30641.

Eyer, P.-A., and E. L. Vargo. 2021. Breeding structure and invasiveness in social insects. Current Opinion in Insect Science 46:24-30.

Faillace, C. A., N. S. Lorusso, and S. Duffy. 2017. Overlooking the smallest matter: Viruses impact biological invasions. Ecology Letters 20:524-538.

Faurot-Daniels, C., W. Glenny, K. F. Daughenbaugh, A. J. McMenamin, L. A. Burkle, and M. L. Flenniken. 2020. Longitudinal monitoring of honey bee colonies reveals dynamic nature of virus abundance and indicates a negative impact of lake Sinai virus 2 on colony health. PLoS One 15:e0237544. 
Feinberg, E. H., and C. P. Hunter. 2003. Transport of dsRNA into cells by the transmembrane protein SID-1. Science 301:1545-1547.

Felden, A., J. Dobelmann, J. W. Baty, J. McCormick, J. Haywood, and P. J. Lester. in preparation. Silencing immunity in the globally invasive Argentine ant as a pest control tool.

Felden, A., C. Paris, D. G. Chapple, A. V. Suarez, N. D. Tsutsui, P. J. Lester, and M. A. M. Gruber. 2019. Native and introduced Argentine ant populations are characterised by distinct transcriptomic signatures associated with behaviour and immunity. NeoBiota 49:105-126.

Fidler, D. P. 1996. Globalization, international law, and emerging infectious diseases. Emerging infectious diseases 2:77-84.

Figueroa, L. L., M. Blinder, C. Grincavitch, A. Jelinek, E. K. Mann, L. A. Merva, L. E. Metz, A. Y. Zhao, R. E. Irwin, S. H. McArt, and L. S. Adler. 2019. Bee pathogen transmission dynamics: Deposition, persistence and acquisition on flowers. Proceedings of the Royal Society B: Biological Sciences 286:20190603.

Flenniken, M. L., and R. Andino. 2013. Non-specific dsRNA-mediated antiviral response in the honey bee. PLoS One 8:e77263.

Forêt, S., and R. Maleszka. 2006. Function and evolution of a gene family encoding odorant binding-like proteins in a social insect, the honey bee (Apis mellifera). Genome Research 16:1404-1413.

Fountain-Jones, N. M., W. D. Pearse, L. E. Escobar, A. Alba-Casals, S. Carver, T. J. Davies, S. Kraberger, M. Papes, K. Vandegrift, K. Worsley-Tonks, and M. E. Craft. 2018. Towards an eco-phylogenetic framework for infectious disease ecology. Biological Reviews 93:950-970.

Fürst, M. A., D. P. McMahon, J. L. Osborne, R. J. Paxton, and M. J. Brown. 2014. Disease associations between honeybees and bumblebees as a threat to wild pollinators. Nature 506:364-366.

Galbraith, D. A., Z. L. Fuller, A. M. Ray, A. Brockmann, M. Frazier, M. W. Gikungu, J. F. I. Martinez, K. M. Kapheim, J. T. Kerby, S. D. Kocher, O. Losyev, E. Muli, H. M. Patch, C. Rosa, J. M. Sakamoto, S. Stanley, A. D. Vaudo, and C. M. Grozinger. 2018. Investigating the viral ecology of global bee communities with high-throughput metagenomics. Scientific Reports 8:8879.

Galbraith, D. A., X. Yang, E. L. Niño, S. Yi, and C. Grozinger. 2015. Parallel epigenomic and transcriptomic responses to viral infection in honey bees (Apis mellifera). PLOS Pathogens 11:e1004713. 
Gallagher, L. M., R. J. Hall, H. Pragert, and Q. H. Fan. 2018. Bee pathogen programme at MPI. Pages 10 - 15 The New Zealand Beekeeper Journal. Apiculture New Zealand, Wellinton.

Gallai, N., J. M. Salles, J. Settele, and B. E. Vaissiere. 2009. Economic valuation of the vulnerability of world agriculture confronted with pollinator decline. Ecological Economics 68:810-821.

Gao, Z., M. Wang, D. Blair, Y. Zheng, and Y. Dou. 2014. Phylogenetic analysis of the endoribonuclease dicer family. PLoS One 9:e95350.

Garbian, Y., E. Maori, H. Kalev, S. Shafir, and I. Sela. 2012. Bidirectional transfer of RNAi between honey bee and Varroa destructor: Varroa gene silencing reduces Varroa population. PLOS Pathogens 8:e1003035.

Garibaldi, L. A., I. Steffan-Dewenter, R. Winfree, M. A. Aizen, R. Bommarco, S. A. Cunningham, C. Kremen, L. G. Carvalheiro, L. D. Harder, O. Afik, I. Bartomeus, F. Benjamin, V. Boreux, D. Cariveau, N. P. Chacoff, J. H. Dudenhoffer, B. M. Freitas, J. Ghazoul, S. Greenleaf, J. Hipolito, A. Holzschuh, B. Howlett, R. Isaacs, S. K. Javorek, C. M. Kennedy, K. M. Krewenka, S. Krishnan, Y. Mandelik, M. M. Mayfield, I. Motzke, T. Munyuli, B. A. Nault, M. Otieno, J. Petersen, G. Pisanty, S. G. Potts, R. Rader, T. H. Ricketts, M. Rundlof, C. L. Seymour, C. Schuepp, H. Szentgyorgyi, H. Taki, T. Tscharntke, C. H. Vergara, B. F. Viana, T. C. Wanger, C. Westphal, N. Williams, and A. M. Klein. 2013. Wild pollinators enhance fruit set of crops regardless of honey bee abundance. Science 339:1608-1611.

Garigliany, M., B. Taminiau, N. El Agrebi, D. Cadar, G. Gilliaux, M. Hue, D. Desmecht, G. Daube, A. Linden, F. Farnir, M. De Proft, and C. Saegerman. 2017. Moku Virus in invasive Asian hornets, Belgium, 2016. Emerging infectious diseases 23:2109-2112.

Garnas, J., E. Groden, and F. A. Drummond. 2014. Mechanisms of competitive displacement of native ant fauna by invading Myrmica rubra (Hymenoptera: Formicidae) populations. Environmental Entomology 43:1496-1506.

Gibbons, D., C. Morrissey, and P. Mineau. 2015. A review of the direct and indirect effects of neonicotinoids and fipronil on vertebrate wildlife. Environmental Science and Pollution Research 22:103-118.

Gisder, S., P. Aumeier, and E. Genersch. 2009. Deformed wing virus: Replication and viral load in mites (Varroa destructor). Journal of General Virology 90:463-467.

Gisder, S., and E. Genersch. 2017. Viruses of commercialized insect pollinators. Journal of Invertebrate Pathology 147:51-59.

Gisder, S., N. Mockel, D. Eisenhardt, and E. Genersch. 2018. In vivo evolution of viral virulence: Switching of deformed wing virus between hosts results in virulence changes and sequence shifts. Environmental Microbiology 20:4612-4628. 
Goulson, D. 2019. The insect apocalypse, and why it matters. Current Biology 29:R967-R971.

Goulson, D., E. Nicholls, C. Botías, and E. L. Rotheray. 2015. Bee declines driven by combined stress from parasites, pesticides, and lack of flowers. Science 347:1255957.

Grabherr, M. G., B. J. Haas, M. Yassour, J. Z. Levin, D. A. Thompson, I. Amit, X. Adiconis, L. Fan, R. Raychowdhury, Q. Zeng, Z. Chen, E. Mauceli, N. Hacohen, A. Gnirke, N. Rhind, F. di Palma, B. W. Birren, C. Nusbaum, K. Lindblad-Toh, N. Friedman, and A. Regev. 2011. Full-length transcriptome assembly from RNA-Seq data without a reference genome. Nature Biotechnology 29:644-652.

Granberg, F., M. Vicente-Rubiano, C. Rubio-Guerri, O. E. Karlsson, D. Kukielka, S. Belák, and J. M. Sánchez-Vizcaíno. 2013. Metagenomic detection of viral pathogens in Spanish honeybees: Co-infection by aphid lethal paralysis, Israel acute paralysis and lake Sinai viruses. PLoS One 8:e57459.

Gray, A., N. Adjlane, A. Arab, A. Ballis, V. Brusbardis, J.-D. Charrière, R. Chlebo, M. F. Coffey, B. Cornelissen, C. Amaro da Costa, B. Dahle, J. Danihlík, M. M. Dražić, G. Evans, M. Fedoriak, I. Forsythe, A. Gajda, D. C. de Graaf, A. Gregorc, I. Ilieva, J. Johannesen, L. Kauko, P. Kristiansen, M. Martikkala, R. Martín-Hernández, C. A. Medina-Flores, F. Mutinelli, S. Patalano, A. Raudmets, G. S. Martin, V. Soroker, J. Stevanovic, A. Uzunov, F. Vejsnaes, A. Williams, M. Zammit-Mangion, and R. Brodschneider. 2020. Honey bee colony winter loss rates for 35 countries participating in the COLOSS survey for winter 2018-2019, and the effects of a new queen on the risk of colony winter loss. Journal of Apicultural Research 59:744-751.

Graystock, P., D. Goulson, and W. O. H. Hughes. 2015. Parasites in bloom: Flowers aid dispersal and transmission of pollinator parasites within and between bee species. Proceedings of the Royal Society B: Biological Sciences 282:20151371.

Graystock, P., K. Yates, S. E. F. Evison, B. Darvill, D. Goulson, W. O. H. Hughes, and J. Osborne. 2013. The Trojan hives: Pollinator pathogens, imported and distributed in bumblebee colonies. Journal of Applied Ecology 50:1207-1215.

Green, O. 1990. Entomologist sets new record at Mt Smart for Iridomyrmex humilis established in New Zealand. Weta 13:14-16.

Grozinger, C. M., and M. L. Flenniken. 2019. Bee viruses: Ecology, pathogenicity, and impacts. Annual Review of Entomology 64:205-226.

Grozinger, C. M., and A. Zayed. 2020. Improving bee health through genomics. Nature Reviews Genetics 21:277-291.

Gruber, M. A. M., A. R. Burne, K. L. Abbott, R. J. Pierce, and P. J. Lester. 2013. Population decline but increased distribution of an invasive ant genotype on a Pacific atoll. Biological Invasions 15:599-612. 
Gruber, M. A. M., M. Cooling, J. W. Baty, K. Buckley, A. Friedlander, O. Quinn, J. Russell, A. Sebastien, and P. J. Lester. 2017. Single-stranded RNA viruses infecting the invasive Argentine ant, Linepithema humile. Scientific Reports 7:3304.

Guu, T. S., W. Zheng, and Y. J. Tao. 2012. Bunyavirus: Structure and replication. Pages 245266 in M. G. Rossmann and V. B. Rao, editors. Viral Molecular Machines. Springer.

Guy, P. L., and P. J. Gerard. 2018. First record of White clover cryptic virus-2 in New Zealand. Australasian Plant Disease Notes 13:43.

Hajek, A. E., and J. Eilenberg. 2018. An introduction to biological control.in A. E. Hajek, editor. Natural Enemies. Cambridge University Press, Cambridge.

Hallmann, C. A., M. Sorg, E. Jongejans, H. Siepel, N. Hofland, H. Schwan, W. Stenmans, A. Muller, H. Sumser, T. Horren, D. Goulson, and H. de Kroon. 2017. More than 75 percent decline over 27 years in total flying insect biomass in protected areas. PLoS One 12:e0185809.

Hamilton, C., B. T. Lejeune, and R. B. Rosengaus. 2011. Trophallaxis and prophylaxis: Social immunity in the carpenter ant Camponotus pennsylvanicus. Biology Letters 7:89-92.

Hannon, G. J. 2002. RNA interference. Nature 418:244-251.

Harris, R. J. 2002. Potential impact of the Argentine ant (Linepithema humile) in New Zealand and options for its control. Department of Conservation, Wellington, New Zealand.

Hartley, S., and P. J. Lester. 2003. Temperature-dependent development of the Argentine ant, Linepithema humile (Mayr) (Hymenoptera: Formicidae): A degree-day model with implications for range limits in New Zealand. New Zealand Entomologist 26:91-100.

Hatfill, S., C. Williamson, R. Kirby, and M. Von Wechmar. 1990. Identification and localization of aphid lethal paralysis virus particles in thin tissue sections of the Rhopalosiphum padi aphid by in situ nucleic acid hybridization. Journal of Invertebrate Pathology 55:265-271.

Henry, M., M. Béguin, F. Requier, O. Rollin, J.-F. Odoux, P. Aupinel, J. Aptel, S. Tchamitchian, and A. Decourtye. 2012. A common pesticide decreases foraging success and survival in honey bees. Science 336:348-350.

Hervé, M. 2018. RVAideMemoire: testing and plotting procedures for biostatistics. R Package Version 0.9-69. Available online at: https:/CRAN.Rproject.org/package=RVAideMemoire.

Highfield, A. C., A. El Nagar, L. C. Mackinder, L. M. Noel, M. J. Hall, S. J. Martin, and D. C. Schroeder. 2009. Deformed wing virus implicated in overwintering honeybee colony losses. Applied and Environmental Microbiology 75:7212-7220. 
Hoffmann, B. D., G. M. Luque, C. Bellard, N. D. Holmes, and C. J. Donlan. 2016. Improving invasive ant eradication as a conservation tool: A review. Biological Conservation 198:37-49.

Hogg, B. N., E. H. Nelson, J. R. Hagler, and K. M. Daane. 2018. Foraging distance of the Argentine ant in California vineyards. Journal of Economic Entomology 111:672-679.

Hölldobler, B., and E. O. Wilson. 1977. The number of queens: An important trait in ant evolution. Naturwissenschaften 64:8-15.

Hölldobler, B., and E. O. Wilson. 1990. The ants. Harvard University Press.

Holmes, E. C. 2009. The evolutionary genetics of emerging viruses. Annual Review of Ecology, Evolution, and Systematics 40:353-372.

Holt, H. L., K. Aronstein, and C. M. Grozinger. 2013. Chronic parasitization by Nosema microsporidia causes global expression changes in core nutritional, metabolic and behavioral pathways in honey bee workers (Apis mellifera). BMC Genomics 14:799.

Holway, D. A. 1999. Competitive mechanisms underlying the displacement of native ants by the invasive Argentine ant. Ecology 80:238-251.

Holway, D. A., L. Lach, A. V. Suarez, N. D. Tsutsui, and T. J. Case. 2002. The causes and consequences of ant invasions. Annual Review of Ecology and Systematics 33:181233.

Huelsenbeck, J. P., and F. Ronquist. 2001. MRBAYES: Bayesian inference of phylogenetic trees. Bioinformatics 17:754-755.

Hulme, P. E. 2009. Trade, transport and trouble: Managing invasive species pathways in an era of globalization. Journal of Applied Ecology 46:10-18.

Hunter, W., J. Ellis, D. VanEngelsdorp, J. Hayes, D. Westervelt, E. Glick, M. Williams, I. Sela, E. Maori, J. Pettis, D. Cox-Foster, and N. Paldi. 2010. Large-scale field application of RNAi technology reducing Israeli acute paralysis virus disease in honey bees (Apis mellifera, Hymenoptera: Apidae). PLOS Pathogens 6:e1001160.

Huryn, V. M. B. 1997. Ecological impacts of introduced honey bees. Quarterly Review of Biology 72:275-297.

Huryn, V. M. B., and H. Moller. 1995. An assessment of the contribution of honey bees (Apis mellifera) to weed reproduction in New Zealand protected natural areas. New Zealand Journal of Ecology 19:111-122.

Huvenne, H., and G. Smagghe. 2010. Mechanisms of dsRNA uptake in insects and potential of RNAi for pest control: A review. Journal of Insect Physiology 56:227-235. 
Ichim, M. C. 2021. The more favorable attitude of the citizens toward GMOs supports a new regulatory framework in the European Union. GM Crops \& Food 12:18-24.

Jackson, D., C. Cornell, B. Luukinen, K. Buhl, and D. Stone. 2009. Fipronil technical fact sheet. National Pesticide Information Center, Oregon State University Extension Services.

Johnson, P. T. J., J. C. de Roode, and A. Fenton. 2015. Why infectious disease research needs community ecology. Science 349:1259504.

Kassambara, A., M. Kosinski, and P. Biecek. 2017. survminer: Drawing survival curves using 'ggplot2'. R Package Version 0.3. Available online at: https://CRAN.Rproject.org/package $=$ survminer.

Keane, R. M., and M. J. Crawley. 2002. Exotic plant invasions and the enemy release hypothesis. Trends in Ecology \& Evolution 17:164-170.

Kearse, M., R. Moir, A. Wilson, S. Stones-Havas, M. Cheung, S. Sturrock, S. Buxton, A. Cooper, S. Markowitz, C. Duran, T. Thierer, B. Ashton, P. Meintjes, and A. Drummond. 2012. Geneious Basic: An integrated and extendable desktop software platform for the organization and analysis of sequence data. Bioinformatics 28:16471649.

Kilpatrick, A. M., P. Daszak, M. J. Jones, P. P. Marra, and L. D. Kramer. 2006. Host heterogeneity dominates West Nile virus transmission. Proceedings of the Royal Society B: Biological Sciences 273:2327-2333.

Kim, D., J. M. Paggi, C. Park, C. Bennett, and S. L. Salzberg. 2019. Graph-based genome alignment and genotyping with HISAT2 and HISAT-genotype. Nature Biotechnology 37:907-915.

Kindt, R., and R. Coe. 2005. Tree diversity analysis: A manual and software for common statistical methods for ecological and biodiversity studies. World Agroforestry Centre (ICRAF).

Kingsolver, M. B., Z. Huang, and R. W. Hardy. 2013. Insect antiviral innate immunity: Pathways, effectors, and connections. Journal of Molecular Biology 425:4921-4936.

Klein, A. M., B. E. Vaissiere, J. H. Cane, I. Steffan-Dewenter, S. A. Cunningham, C. Kremen, and T. Tscharntke. 2007. Importance of pollinators in changing landscapes for world crops. Proceedings of the Royal Society B: Biological Sciences 274:303-313.

Kolde, R. 2019. pheatmap: Pretty Heatmaps. R package version 1.0.12. Available online at: https://CRAN.R-project.org/package=pheatmap. 
Kulhanek, K., N. Steinhauer, K. Rennich, D. M. Caron, R. R. Sagili, J. S. Pettis, J. D. Ellis, M. E. Wilson, J. T. Wilkes, D. R. Tarpy, R. Rose, K. Lee, J. Rangel, and D. vanEngelsdorp. 2017. A national survey of managed honey bee 2015-2016 annual colony losses in the USA. Journal of Apicultural Research 56:328-340.

Kumar, S., G. Stecher, M. Li, C. Knyaz, and K. Tamura. 2018. MEGA X: Molecular Evolutionary Genetics Analysis across computing platforms. Molecular Biology and Evolution 35:1547-1549.

Lach, L. 2007. Argentine ants displace floral arthropods in a biodiversity hotspot. Diversity and Distributions 14:281-290.

Lafferty, K. D. 2010. Interacting parasites. Science 330:187-188.

Le Sage, M. J., B. D. Towey, and J. L. Brunner. 2019. Do scavengers prevent or promote disease transmission? The effect of invertebrate scavenging on Ranavirus transmission. Functional Ecology 33:1342-1350.

Lee, K.-A., and W.-J. Lee. 2018. Immune-metabolic interactions during systemic and enteric infection in Drosophila. Current Opinion in Insect Science 29:21-26.

Lee, Y. S., K. Nakahara, J. W. Pham, K. Kim, Z. He, E. J. Sontheimer, and R. W. Carthew. 2004. Distinct roles for Drosophila dicer-1 and dicer-2 in the siRNA/miRNA silencing pathways. Cell 117:69-81.

Lello, J., B. Boag, A. Fenton, I. R. Stevenson, and P. J. Hudson. 2004. Competition and mutualism among the gut helminths of a mammalian host. Nature 428:840-844.

Lemaitre, B., J. M. Reichhart, and J. A. Hoffmann. 1997. Drosophila host defense: Differential induction of antimicrobial peptide genes after infection by various classes of microorganisms. Proceedings of the National Academy of Sciences 94:14614-14619.

Lembo, T., K. Hampson, D. T. Haydon, M. Craft, A. Dobson, J. Dushoff, E. Ernest, R. Hoare, M. Kaare, T. Mlengeya, C. Mentzel, and S. Cleaveland. 2008. Exploring reservoir dynamics: A case study of rabies in the Serengeti ecosystem. Journal of Applied Ecology 45:1246-1257.

Lemoine, N. P., A. Hoffman, A. J. Felton, L. Baur, F. Chaves, J. Gray, Q. Yu, and M. D. Smith. 2016. Underappreciated problems of low replication in ecological field studies. Ecology 97:2554-2561.

Leonard, S. P., J. E. Powell, J. Perutka, P. Geng, L. C. Heckmann, R. D. Horak, B. W. Davies, A. D. Ellington, J. E. Barrick, and N. A. Moran. 2020. Engineered symbionts activate honey bee immunity and limit pathogens. Science 367:573-576. 
Leroy, E. M., B. Kumulungui, X. Pourrut, P. Rouquet, A. Hassanin, P. Yaba, A. Delicat, J. T. Paweska, J. P. Gonzalez, and R. Swanepoel. 2005. Fruit bats as reservoirs of Ebola virus. Nature 438:575-576.

Lester, P. J., K. H. Buick, J. W. Baty, A. Felden, and J. Haywood. 2019. Different bacterial and viral pathogens trigger distinct immune responses in a globally invasive ant. Scientific Reports 9:5780.

Lester, P. J., and M. A. M. Gruber. 2016. Booms, busts and population collapses in invasive ants. Biological Invasions 18:3091-3101.

Lester, P. J., A. Sébastien, A. V. Suarez, R. F. Barbieri, and M. A. M. Gruber. 2017. Symbiotic bacterial communities in ants are modified by invasion pathway bottlenecks and alter host behavior. Ecology 98:861-874.

Levitt, A. L., R. Singh, D. L. Cox-Foster, E. Rajotte, K. Hoover, N. Ostiguy, and E. C. Holmes. 2013. Cross-species transmission of honey bee viruses in associated arthropods. Virus Research 176:232-240.

Li, C. X., M. Shi, J. H. Tian, X. D. Lin, Y. J. Kang, L. J. Chen, X. C. Qin, J. Xu, E. C. Holmes, and Y. Z. Zhang. 2015. Unprecedented genomic diversity of RNA viruses in arthropods reveals the ancestry of negative-sense RNA viruses. Elife 4:e 05378.

Li, F., and S. W. Ding. 2006. Virus counterdefense: Diverse strategies for evading the RNAsilencing immunity. Annual Review of Microbiology 60:503-531.

Li, H., B. Handsaker, A. Wysoker, T. Fennell, J. Ruan, N. Homer, G. Marth, G. Abecasis, R. Durbin, and 1000 Genome Project Data Processing Subgroup. 2009. The Sequence Alignment/Map format and SAMtools. Bioinformatics 25:2078-2079.

Li, H., B. Yin, S. Wang, Q. Fu, B. Xiao, K. Lü, J. He, and C. Li. 2018. RNAi screening identifies a new Toll from shrimp Litopenaeus vannamei that restricts WSSV infection through activating Dorsal to induce antimicrobial peptides. PLoS Pathogens 14:e1007109.

Li, J. L., R. S. Cornman, J. D. Evans, J. S. Pettis, Y. Zhao, C. Murphy, W. J. Peng, J. Wu, M. Hamilton, H. F. Boncristiani, L. Zhou, J. Hammond, and Y. P. Chen. 2014. Systemic spread and propagation of a plant-pathogenic virus in European honeybees, Apis mellifera. mBio 5:e0898-00813.

Liu, S., D. Vijayendran, and B. C. Bonning. 2011. Next generation sequencing technologies for insect virus discovery. Viruses 3:1849-1869.

Liu, S., D. Vijayendran, J. Carrillo-Tripp, W. A. Miller, and B. C. Bonning. 2014. Analysis of new aphid lethal paralysis virus (ALPV) isolates suggests evolution of two ALPV species. Journal of General Virology 95:2809-2819. 
Livak, K. J., and T. D. Schmittgen. 2001. Analysis of relative gene expression data using realtime quantitative PCR and the $2^{-\Delta \Delta C T}$ method. Methods 25:402-408.

Loope, K. J., J. W. Baty, P. J. Lester, and E. E. Wilson Rankin. 2019. Pathogen shifts in a honeybee predator following the arrival of the Varroa mite. Proceedings of the Royal Society B: Biological Sciences 286:20182499.

Lopez, S. B. G., V. Guimarães-Ribeiro, J. V. G. Rodriguez, F. A. P. S. Dorand, T. S. Salles, T. E. Sá-Guimarães, E. S. L. Alvarenga, A. C. A. Melo, R. V. Almeida, and M. F. Moreira. 2019. RNAi-based bioinsecticide for Aedes mosquito control. Scientific Reports 9:4038.

Love, M. I., W. Huber, and S. Anders. 2014. Moderated estimation of fold change and dispersion for RNA-seq data with DESeq2. Genome Biology 15:550.

Lowe, S., M. Browne, S. Boudjelas, and M. De Poorter. 2000. 100 of the world's worst invasive alien species : A selection from the global invasive species database. Invasive Species Specialist Group, Auckland.

Lucht, J. M. 2015. Public acceptance of plant biotechnology and GM crops. Viruses 7:42544281.

Macedo, P. A., J. Wu, and M. D. Ellis. 2002. Using inert dusts to detect and assess Varroa infestations in honey bee colonies. Journal of Apicultural Research 41:3-7.

Manley, R., M. Boots, and L. Wilfert. 2015. Emerging viral disease risk to pollinating insects: Ecological, evolutionary and anthropogenic factors. Journal of Applied Ecology 52:331-340.

Manley, R., B. Temperton, M. Boots, and L. Wilfert. 2020. Contrasting impacts of a novel specialist vector on multihost viral pathogen epidemiology in wild and managed bees. Molecular Ecology 29:380-393.

Manley, R., B. Temperton, T. Doyle, D. Gates, S. Hedges, M. Boots, and L. Wilfert. 2019. Knock-on community impacts of a novel vector: Spillover of emerging DWV-B from Varroa-infested honeybees to wild bumblebees. Ecology Letters 22:1306-1315.

Marco Antonio, D. S., K. R. Guidugli-Lazzarini, A. M. do Nascimento, Z. L. P. Simões, and K. Hartfelder. 2008. RNAi-mediated silencing of vitellogenin gene function turns honeybee (Apis mellifera) workers into extremely precocious foragers. Naturwissenschaften 95:953-961.

Martin, S. J., and L. E. Brettell. 2019. Deformed wing virus in honeybees and other insects. Annual Review of Virology 6:49-69. 
Martin, S. J., A. C. Highfield, L. Brettell, E. M. Villalobos, G. E. Budge, M. Powell, S. Nikaido, and D. C. Schroeder. 2012. Global honey bee viral landscape altered by a parasitic mite. Science 336:1304-1306.

Mat Jalaluddin, N. S., R. Y. Othman, and J. A. Harikrishna. 2019. Global trends in research and commercialization of exogenous and endogenous RNAi technologies for crops. Critical Reviews in Biotechnology 39:67-78.

McArt, S. H., H. Koch, R. E. Irwin, and L. S. Adler. 2014. Arranging the bouquet of disease: floral traits and the transmission of plant and animal pathogens. Ecology Letters 17:624-636.

McCallum, H., and A. Dobson. 1995. Detecting disease and parasite threats to endangered species and ecosystems. Trends in Ecology \& Evolution 10:190-194.

McFadden, A. M. J., K.-M. Tham, M. Stevenson, M. Goodwin, H. Pharo, B. Taylor, G. Munro, K. Owen, L. Peacock, W. L. Stanslawek, and M. Stone. 2015. Israeli acute paralysis virus not detected in Apis mellifera in New Zealand in a national survey. Journal of Apicultural Research 53:520-527.

McMahon, D. P., M. A. Furst, J. Caspar, P. Theodorou, M. J. F. Brown, and R. J. Paxton. 2015. A sting in the spit: Widespread cross-infection of multiple RNA viruses across wild and managed bees. Journal of Animal Ecology 84:615-624.

McMahon, D. P., L. Wilfert, R. J. Paxton, and M. J. Brown. 2018. Emerging viruses in bees: From molecules to ecology. Advances in Virus Research 101:251-291.

McMenamin, A. J., and M. L. Flenniken. 2018. Recently identified bee viruses and their impact on bee pollinators. Current Opinion in Insect Science 26:120-129.

McMenamin, A. J., and E. Genersch. 2015. Honey bee colony losses and associated viruses. Current Opinion in Insect Science 8:121-129.

Meeus, I., J. R. de Miranda, D. C. de Graaf, F. Wackers, and G. Smagghe. 2014. Effect of oral infection with Kashmir bee virus and Israeli acute paralysis virus on bumblebee (Bombus terrestris) reproductive success. Journal of Invertebrate Pathology 121:64-69.

Mideo, N. 2009. Parasite adaptations to within-host competition. Trends in Parasitology 25:261-268.

Miller, W. A., J. Carrillo-Tripp, B. C. Bonning, A. G. Dolezal, and A. L. Toth. 2014. Conclusive evidence of replication of a plant virus in honeybees is lacking. mBio 5:e00985-00914.

Ministry of Primary Industries. 2018. MPI 2017 apiculture monitoring programme report. 9781-77665-778-0. 
Moller, H. 1996. Lessons for invasion theory from social insects. Biological Conservation 78:125-142.

Mondet, F., J. R. de Miranda, A. Kretzschmar, Y. Le Conte, and A. R. Mercer. 2014. On the front line: Quantitative virus dynamics in honeybee (Apis mellifera L.) colonies along a new expansion front of the parasite Varroa destructor. PLoS Pathogens 10:e1004323.

Mordecai, G. J., L. E. Brettell, S. J. Martin, D. Dixon, I. M. Jones, and D. C. Schroeder. 2016a. Superinfection exclusion and the long-term survival of honey bees in Varroa-infested colonies. ISME Journal 10:1182-1191.

Mordecai, G. J., L. E. Brettell, P. Pachori, E. M. Villalobos, S. J. Martin, I. M. Jones, and D. C. Schroeder. 2016b. Moku virus; A new Iflavirus found in wasps, honey bees and Varroa. Scientific Reports 6:34983.

Murray, E. A., J. Burand, N. Trikoz, J. Schnabel, H. Grab, and B. N. Danforth. 2019. Viral transmission in honey bees and native bees, supported by a global black queen cell virus phylogeny. Environmental Microbiology 21:972-983.

Mutti, N. S., A. G. Dolezal, F. Wolschin, J. S. Mutti, K. S. Gill, and G. V. Amdam. 2011. IRS and TOR nutrient-signaling pathways act via juvenile hormone to influence honey bee caste fate. The Journal of Experimental Biology 214:3977-3984.

Natsopoulou, M. E., D. P. McMahon, V. Doublet, J. Bryden, and R. J. Paxton. 2015. Interspecific competition in honeybee intracellular gut parasites is asymmetric and favours the spread of an emerging infectious disease. Proceedings of the Royal Society B: Biological Sciences 282:20141896.

Natsopoulou, M. E., D. P. McMahon, V. Doublet, E. Frey, P. Rosenkranz, and R. J. Paxton. 2017. The virulent, emerging genotype B of Deformed wing virus is closely linked to overwinter honeybee worker loss. Scientific Reports 7:5242.

Nazzi, F., S. P. Brown, D. Annoscia, F. Del Piccolo, G. Di Prisco, P. Varricchio, G. Della Vedova, F. Cattonaro, E. Caprio, and F. Pennacchio. 2012. Synergistic parasitepathogen interactions mediated by host immunity can drive the collapse of honeybee colonies. PLoS Pathogens 8:e1002735.

Nazzi, F., and F. Pennacchio. 2014. Disentangling multiple interactions in the hive ecosystem. Trends in Parasitology 30:556-561.

Nazzi, F., and F. Pennacchio. 2018. Honey bee antiviral immune barriers as affected by multiple stress factors: A novel paradigm to interpret colony health decline and collapse. Viruses 10:159.

Nunes, F. M. F., A. C. Aleixo, A. R. Barchuk, A. D. Bomtorin, C. M. Grozinger, and Z. L. P. Simões. 2013. Non-target effects of green fluorescent protein (GFP)-derived double- 
stranded RNA (dsRNA-GFP) used in honey bee RNA interference (RNAi) assays. Insects 4:90-103.

Nunn, C. L., F. Jordán, C. M. McCabe, J. L. Verdolin, and J. H. Fewell. 2015. Infectious disease and group size: More than just a numbers game. Philosophical Transactions of the Royal Society B: Biological Sciences 370:20140111.

O’Neal, S. T., T. D. Anderson, and J. Y. Wu-Smart. 2018. Interactions between pesticides and pathogen susceptibility in honey bees. Current Opinion in Insect Science 26:57-62.

Obbard, D. J., F. M. Jiggins, D. L. Halligan, and T. J. Little. 2006. Natural selection drives extremely rapid evolution in antiviral RNAi genes. Current Biology 16:580-585.

Oerke, E. C., and H. W. Dehne. 2004. Safeguarding production-losses in major crops and the role of crop protection. Crop Protection 23:275-285.

Oksanen, J., F. G. Blanchet, M. Friendly, R. Kindt, P. Legendre, D. McGlinn, M. P. R., R. O’hara, G. L. Simpson, P. Solymos, M. H. H. Stevens, S. E, and H. Wagner. 2017. Vegan: Community ecology package. R package version 2.4-2. Available online at: https://CRAN.R-project.org/package=vegan.

Oldroyd, B. P. 1999. Coevolution while you wait: Varroa jacobsoni, a new parasite of western honeybees. Trends in Ecology \& Evolution 14:312-315.

Palmer, M. V. 2013. Mycobacterium bovis: Characteristics of wildlife reservoir hosts. Transboundary and Emerging Diseases 60:1-13.

Palmer, S., and O. R. Mercier. 2020. Biotechnologies in pest wasp control: Taking the sting out of pest management for Māori businesses? New Genetics and Society:1-23.

Park, S., Y. H. Jo, K. B. Park, H. J. Ko, C. E. Kim, Y. M. Bae, B. Kim, S. A. Jun, I. S. Bang, Y. S. Lee, Y. J. Kim, and Y. S. Han. 2019. TmToll-7 Plays a crucial role in innate immune responses against gram-negative bacteria by regulating 5 AMP genes in Tenebrio molitor. Frontiers in Immunology 10:310.

Parker, J., A. Rambaut, and O. G. Pybus. 2008. Correlating viral phenotypes with phylogeny: Accounting for phylogenetic uncertainty. Infection, Genetics and Evolution 8:239-246.

Parrish, C. R., E. C. Holmes, D. M. Morens, E.-C. Park, D. S. Burke, C. H. Calisher, C. A. Laughlin, L. J. Saif, and P. Daszak. 2008. Cross-species virus transmission and the emergence of new epidemic diseases. Microbiology and Molecular Biology Reviews 72:457-470.

Pascall, D. J., M. C. Tinsley, D. J. Obbard, and L. Wilfert. 2019. Host evolutionary history predicts virus prevalence across bumblebee species. bioRxiv:498717. 
Payne, A. N., T. F. Shepherd, and J. Rangel. 2020. The detection of honey bee (Apis mellifera)associated viruses in ants. Scientific Reports 10:2923.

Pertea, M., G. M. Pertea, C. M. Antonescu, T.-C. Chang, J. T. Mendell, and S. L. Salzberg. 2015. StringTie enables improved reconstruction of a transcriptome from RNA-seq reads. Nature Biotechnology 33:290-295.

Pisa, L. W., V. Amaral-Rogers, L. P. Belzunces, J. M. Bonmatin, C. A. Downs, D. Goulson, D. P. Kreutzweiser, C. Krupke, M. Liess, M. McField, C. A. Morrissey, D. A. Noome, J. Settele, N. Simon-Delso, J. D. Stark, J. P. Van der Sluijs, H. Van Dyck, and M. Wiemers. 2015. Effects of neonicotinoids and fipronil on non-target invertebrates. Environmental Science and Pollution Research 22:68-102.

Porter, S. D., S. M. Valles, and R. M. Pereira. 2016. Scavenging crickets (Orthoptera: Gryllidae) transmit Solenopsis invicta virus 3 to red imported fire ant (Hymenoptera: Formicidae) colonies. Florida Entomologist 99:811-812.

Posada-Florez, F., A. K. Childers, M. C. Heerman, N. I. Egekwu, S. C. Cook, Y. Chen, J. D. Evans, and E. V. Ryabov. 2019. Deformed wing virus type A, a major honey bee pathogen, is vectored by the mite Varroa destructor in a non-propagative manner. Scientific Reports 9:12445.

Potts, S. G., J. C. Biesmeijer, C. Kremen, P. Neumann, O. Schweiger, and W. E. Kunin. 2010. Global pollinator declines: Trends, impacts and drivers. Trends in Ecology \& Evolution 25:345-353.

R Core Team. 2014. R: A language and environment for statistical computing. Vienna, Austria: R Foundation for Statistical Computing. Retrieved from: https://www.R-project.org.

Rader, R., I. Bartomeus, L. A. Garibaldi, M. P. D. Garratt, B. G. Howlett, R. Winfree, S. A. Cunningham, M. M. Mayfield, A. D. Arthur, G. K. S. Andersson, R. Bommarco, C. Brittain, L. G. Carvalheiro, N. P. Chacoff, M. H. Entling, B. Foully, B. M. Freitas, B. Gemmill-Herren, J. Ghazoul, S. R. Griffin, C. L. Gross, L. Herbertsson, F. Herzog, J. Hipólito, S. Jaggar, F. Jauker, A.-M. Klein, D. Kleijn, S. Krishnan, C. Q. Lemos, S. A. M. Lindström, Y. Mandelik, V. M. Monteiro, W. Nelson, L. Nilsson, D. E. Pattemore, N. de O. Pereira, G. Pisanty, S. G. Potts, M. Reemer, M. Rundlöf, C. S. Sheffield, J. Scheper, C. Schüepp, H. G. Smith, D. A. Stanley, J. C. Stout, H. Szentgyörgyi, H. Taki, C. H. Vergara, B. F. Viana, and M. Woyciechowski. 2016. Non-bee insects are important contributors to global crop pollination. Proceedings of the National Academy of Sciences 113:146-151.

Ramsey, S. D., R. Ochoa, G. Bauchan, C. Gulbronson, J. D. Mowery, A. Cohen, D. Lim, J. Joklik, J. M. Cicero, J. D. Ellis, D. Hawthorne, and D. vanEngelsdorp. 2019. Varroa destructor feeds primarily on honey bee fat body tissue and not hemolymph. Proceedings of the National Academy of Sciences 116:1792-1801.

Ratnieks, F. L., and N. L. Carreck. 2010. Clarity on honey bee collapse? Science 327:152-153. 
Ravoet, J., L. De Smet, I. Meeus, G. Smagghe, T. Wenseleers, and D. C. de Graaf. 2014. Widespread occurrence of honey bee pathogens in solitary bees. Journal of Invertebrate Pathology 122:55-58.

Ravoet, J., J. Maharramov, I. Meeus, L. De Smet, T. Wenseleers, G. Smagghe, and D. C. de Graaf. 2013. Comprehensive bee pathogen screening in Belgium reveals Crithidia mellificae as a new contributory factor to winter mortality. PLoS One 8:e72443.

Remnant, E. J., N. Mather, T. L. Gillard, B. Yagound, and M. Beekman. 2019. Direct transmission by injection affects competition among RNA viruses in honeybees. Proceedings of the Royal Society B: Biological Sciences 286:20182452.

Remnant, E. J., M. Shi, G. Buchmann, T. Blacquiere, E. C. Holmes, M. Beekman, and A. Ashe. 2017. A diverse range of novel RNA viruses in geographically distinct honey bee populations. Journal of Virology 91:e00158-00117.

Rieux, A., and F. Balloux. 2016. Inferences from tip-calibrated phylogenies: A review and a practical guide. Molecular Ecology 25:1911-1924.

Rigaud, T., M. J. Perrot-Minnot, and M. J. Brown. 2010. Parasite and host assemblages: Embracing the reality will improve our knowledge of parasite transmission and virulence. Proceedings of the Royal Society B: Biological Sciences 277:3693-3702.

Roberts, J. M. K., D. L. Anderson, and P. A. Durr. 2018. Metagenomic analysis of Varroa-free Australian honey bees (Apis mellifera) shows a diverse Picornavirales virome. Journal of General Virology 99:818-826.

Rothan, H. A., and S. N. Byrareddy. 2020. The epidemiology and pathogenesis of coronavirus disease (COVID-19) outbreak. Journal of Autoimmunity 109:102433.

Roura-Pascual, N., C. Hui, T. Ikeda, G. Leday, D. M. Richardson, S. Carpintero, X. Espadaler, C. Gómez, B. Guénard, and S. Hartley. 2011. Relative roles of climatic suitability and anthropogenic influence in determining the pattern of spread in a global invader. Proceedings of the National Academy of Sciences 108:220-225.

Roura-Pascual, N., A. V. Suarez, C. Gomez, P. Pons, Y. Touyama, A. L. Wild, and A. T. Peterson. 2004. Geographical potential of Argentine ants (Linepithema humile Mayr) in the face of global climate change. Proceedings of the Royal Society B: Biological Sciences 271:2527-2535.

Runckel, C., M. L. Flenniken, J. C. Engel, J. G. Ruby, D. Ganem, R. Andino, and J. L. DeRisi. 2011. Temporal analysis of the honey bee microbiome reveals four novel viruses and seasonal prevalence of known viruses, Nosema, and Crithidia. PLoS One 6:e20656.

Ryabov, E. V., J. M. Fannon, J. D. Moore, G. R. Wood, and D. J. Evans. 2016. The Iflaviruses Sacbrood virus and deformed wing virus evoke different transcriptional responses in 
the honeybee which may facilitate their horizontal or vertical transmission. PeerJ 4:e1591.

Ryabov, E. V., G. R. Wood, J. M. Fannon, J. D. Moore, J. C. Bull, D. Chandler, A. Mead, N. Burroughs, and D. J. Evans. 2014. A virulent strain of deformed wing virus (DWV) of honeybees (Apis mellifera) prevails after Varroa destructor-mediated, or in vitro, transmission. PLoS Pathogens 10:e1004230.

Sánchez-Bayo, F., and K. A. G. Wyckhuys. 2019. Worldwide decline of the entomofauna: A review of its drivers. Biological Conservation 232:8-27.

Sanchez, A., T. G. Ksiazek, P. E. Rollin, C. J. Peters, S. T. Nichol, A. S. Khan, and B. W. Mahy. 1995. Reemergence of Ebola virus in Africa. Emerging infectious diseases 1:9697.

Santamaria, J., E. M. Villalobos, L. E. Brettell, S. Nikaido, J. R. Graham, and S. Martin. 2018. Evidence of Varroa-mediated deformed wing virus spillover in Hawaii. Journal of Invertebrate Pathology 151:126-130.

Schindelin, J., C. T. Rueden, M. C. Hiner, and K. W. Eliceiri. 2015. The ImageJ ecosystem: An open platform for biomedical image analysis. Molecular Reproduction and Development 82:518-529.

Schläppi, D., N. Chejanovsky, O. Yañez, and P. Neumann. 2020. Foodborne transmission and clinical symptoms of honey bee viruses in ants Lasius spp. Viruses 12:321.

Schläppi, D., P. Lattrell, O. Yañez, N. Chejanovsky, and P. Neumann. 2019. Foodborne transmission of deformed wing virus to ants (Myrmica rubra). Insects 10:394.

Schmid-Hempel, R., M. Eckhardt, D. Goulson, D. Heinzmann, C. Lange, S. Plischuk, L. R. Escudero, R. Salathe, J. J. Scriven, and P. Schmid-Hempel. 2014. The invasion of southern South America by imported bumblebees and associated parasites. Journal of Animal Ecology 83:823-837.

Schoonvaere, K., L. De Smet, G. Smagghe, A. Vierstraete, B. P. Braeckman, and D. C. de Graaf. 2016. Unbiased RNA shotgun metagenomics in social and solitary wild bees detects associations with eukaryote parasites and new viruses. PLoS One 11:e0168456.

Schwarz, R. S., N. A. Moran, and J. D. Evans. 2016. Early gut colonizers shape parasite susceptibility and microbiota composition in honey bee workers. Proceedings of the National Academy of Sciences 113:9345-9350.

Seabloom, E. W., E. T. Borer, K. Gross, A. E. Kendig, C. Lacroix, C. E. Mitchell, E. A. Mordecai, and A. G. Power. 2015. The community ecology of pathogens: Coinfection, coexistence and community composition. Ecology Letters 18:401-415. 
Sebastien, A., P. J. Lester, R. J. Hall, J. Wang, N. E. Moore, and M. A. Gruber. 2015. Invasive ants carry novel viruses in their new range and form reservoirs for a honeybee pathogen. Biology Letters 11:20150610.

Seppälä, O., A. Karvonen, E. T. Valtonen, and J. Jokela. 2009. Interactions among co-infecting parasite species: A mechanism maintaining genetic variation in parasites? Proceedings of the Royal Society B: Biological Sciences 276:691-697.

Shi, M., X. D. Lin, J. H. Tian, L. J. Chen, X. Chen, C. X. Li, X. C. Qin, J. Li, J. P. Cao, J. S. Eden, J. Buchmann, W. Wang, J. Xu, E. C. Holmes, and Y. Z. Zhang. 2016. Redefining the invertebrate RNA virosphere. Nature 540:539.

Shipp, J. L., R. Buitenhuis, L. Stobbs, K. Wang, W. S. Kim, and G. Ferguson. 2008. Vectoring of Pepino mosaic virus by bumble-bees in tomato greenhouses. Annals of Applied Biology 153:149-155.

Shukla, J. N., M. Kalsi, A. Sethi, K. E. Narva, E. Fishilevich, S. Singh, K. Mogilicherla, and S. R. Palli. 2016. Reduced stability and intracellular transport of dsRNA contribute to poor RNAi response in lepidopteran insects. RNA Biology 13:656-669.

Sidhu, S. C., and E. E. Wilson Rankin. 2016. Honey bees avoiding ant harassment at flowers using scent cues. Environmental Entomology 45:420-426.

Silverman, J., and R. J. Brightwell. 2008. The Argentine Ant: Challenges in managing an invasive unicolonial pest. Annual Review of Entomology 53:231-252.

Simberloff, D., and L. Gibbons. 2004. Now you see them, now you don't! - Population crashes of established introduced species. Biological Invasions 6:161-172.

Simone-Finstrom, M., M. Walz, and D. R. Tarpy. 2016. Genetic diversity confers colony-level benefits due to individual immunity. Biology Letters 12:20151007.

Singh, R., A. L. Levitt, E. G. Rajotte, E. C. Holmes, N. Ostiguy, D. vanEngelsdorp, W. I. Lipkin, C. W. Depamphilis, A. L. Toth, and D. L. Cox-Foster. 2010. RNA viruses in hymenopteran pollinators: Evidence of inter-taxa virus transmission via pollen and potential impact on non-Apis hymenopteran species. PLoS One 5:e14357.

Sinu, P. A., V. C. Sibisha, M. V. Nikhila Reshmi, K. S. Reshmi, T. V. Jasna, K. Aswathi, and P. P. Megha. 2017. Invasive ant (Anoplolepis gracilipes) disrupts pollination in pumpkin. Biological Invasions 19:2599-2607.

Smith, C. D., A. Zimin, C. Holt, E. Abouheif, R. Benton, E. Cash, V. Croset, C. R. Currie, E. Elhaik, C. G. Elsik, M.-J. Fave, V. Fernandes, J. Gadau, J. D. Gibson, D. Graur, K. J. Grubbs, D. E. Hagen, M. Helmkampf, J.-A. Holley, H. Hu, A. S. I. Viniegra, B. R. Johnson, R. M. Johnson, A. Khila, J. W. Kim, J. Laird, K. A. Mathis, J. A. Moeller, M. C. Muñoz-Torres, M. C. Murphy, R. Nakamura, S. Nigam, R. P. Overson, J. E. Placek, R. Rajakumar, J. T. Reese, H. M. Robertson, C. R. Smith, A. V. Suarez, G. Suen, E. L. 
Suhr, S. Tao, C. W. Torres, E. van Wilgenburg, L. Viljakainen, K. K. O. Walden, A. L. Wild, M. Yandell, J. A. Yorke, and N. D. Tsutsui. 2011. Draft genome of the globally widespread and invasive Argentine ant (Linepithema humile). Proceedings of the National Academy of Sciences 108:5673-5678.

Sorrell, I., A. White, A. B. Pedersen, R. S. Hails, and M. Boots. 2009. The evolution of covert, silent infection as a parasite strategy. Proceedings of the Royal Society B: Biological Sciences 276:2217-2226.

Stahlmann-Brown, P., T. Robertson, and O. Borowik. 2020. Report on the 2019 New Zealand colony loss survey. Ministry for Primary Industries, New Zealand.

Stevenson, M., T. Nunes, J. Sanchez, R. Thornton, J. Reiczigel, J. Robison-Cox, and P. Sebastiani. 2013. epiR: An R package for the analysis of epidemiological data. $\mathrm{R}$ Package Version 1.0-4. Available onlin at: https://CRAN.R-project.org/package=epiR.

Suarez, A. V., D. A. Holway, and T. J. Case. 2001. Patterns of spread in biological invasions dominated by long-distance jump dispersal: Insights from Argentine ants. Proceedings of the National Academy of Sciences 98:1095-1100.

Suarez, A. V., D. A. Holway, D. Liang, N. D. Tsutsui, and T. J. Case. 2002. Spatiotemporal patterns of intraspecific aggression in the invasive Argentine ant. Animal Behaviour 64:697-708.

Suarez, A. V., P. Yeh, and T. J. Case. 2005. Impacts of Argentine ants on avian nesting success. Insectes Sociaux 52:378-382.

Tapia-González, J. M., N. Morfin, J. O. Macías-Macías, A. De la Mora, J. C. Tapia-Rivera, R. Ayala, F. Contreras-Escareño, H. A. Gashout, and E. Guzman-Novoa. 2019. Evidence of presence and replication of honey bee viruses among wild bee pollinators in subtropical environments. Journal of Invertebrate Pathology 168:107256.

Tehel, A., M. J. Brown, and R. J. Paxton. 2016. Impact of managed honey bee viruses on wild bees. Current Opinion in Virology 19:16-22.

Telfer, S., X. Lambin, R. Birtles, P. Beldomenico, S. Burthe, S. Paterson, and M. Begon. 2010. Species interactions in a parasite community drive infection risk in a wildlife population. Science 330:243-246.

Tentcheva, D., L. Gauthier, N. Zappulla, B. Dainat, F. Cousserans, M. E. Colin, and M. Bergoin. 2004. Prevalence and seasonal variations of six bee viruses in Apis mellifera L. and Varroa destructor mite populations in France. Applied and Environmental Microbiology 70:7185-7191.

Therneau, T. 2020. A package for survival analysis in R, R package version 3.1-12. Available online at: https://CRAN.R-project.org/package=survival. 
Thompson, J., D. Higgins, and T. Gibson. 1994. CLUSTALW: Improving the sensitivity of progressive multiple sequence through weighing, position-specific gap penalties and weight matrix choice. Nucleic Acids Research 22:4673-4680.

To, K. K. W., J. F. W. Chan, A. K. L. Tsang, V. C. C. Cheng, and K.-Y. Yuen. 2015. Ebola virus disease: A highly fatal infectious disease reemerging in West Africa. Microbes and Infection 17:84-97.

Torchin, M. E., K. D. Lafferty, A. P. Dobson, V. J. McKenzie, and A. M. Kuris. 2003. Introduced species and their missing parasites. Nature 421:628-630.

Tritschler, M., G. Retschnig, O. Yañez, G. R. Williams, and P. Neumann. 2017. Host sharing by the honey bee parasites Lotmaria passim and Nosema ceranae. Ecology and Evolution 7:1850-1857.

Tsutsui, N. D., and T. J. Case. 2001. Population genetics and colony structure of the Argentine ant (Linepithema humile) in its native and introduced ranges. Evolution 55:976-985.

Tsutsui, N. D., A. V. Suarez, D. A. Holway, and T. J. Case. 2000. Reduced genetic variation and the success of an invasive species. Proceedings of the National Academy of Sciences 97:5948-5953.

Ugelvig, L. V., D. J. C. Kronauer, A. Schrempf, J. Heinze, and S. Cremer. 2010. Rapid antipathogen response in ant societies relies on high genetic diversity. Proceedings of the Royal Society B: Biological Sciences 277:2821-2828.

Ulrich, J., V. A. Dao, U. Majumdar, C. Schmitt-Engel, J. Schwirz, D. Schultheis, N. Ströhlein, N. Troelenberg, D. Grossmann, T. Richter, J. Dönitz, L. Gerischer, G. Leboulle, A. Vilcinskas, M. Stanke, and G. Bucher. 2015. Large scale RNAi screen in Tribolium reveals novel target genes for pest control and the proteasome as prime target. BMC Genomics 16:674.

van Klink, R., D. E. Bowler, K. B. Gongalsky, A. B. Swengel, A. Gentile, and J. M. Chase. 2020. Meta-analysis reveals declines in terrestrial but increases in freshwater insect abundances. Science 368:417-420.

van Rij, R. P., M. C. Saleh, B. Berry, C. Foo, A. Houk, C. Antoniewski, and R. Andino. 2006. The RNA silencing endonuclease Argonaute 2 mediates specific antiviral immunity in Drosophila melanogaster. Genes and Development 20:2985-2995.

Vanbergen, A. J., A. Espíndola, and M. A. Aizen. 2018. Risks to pollinators and pollination from invasive alien species. Nature Ecology \& Evolution 2:16-25.

Vejnovic, B., J. Stevanovic, R. S. Schwarz, N. Aleksic, M. Mirilovic, N. M. Jovanovic, and Z. Stanimirovic. 2018. Quantitative PCR assessment of Lotmaria passim in Apis mellifera colonies co-infected naturally with Nosema ceranae. Journal of Invertebrate Pathology 151:76-81. 
Viljakainen, L., I. Holmberg, S. Abril, and J. Jurvansuu. 2018. Viruses of invasive Argentine ants from the European main supercolony: Characterization, interactions and evolution. Journal of General Virology 99:1129-1140.

Vitousek, P. M., H. A. Mooney, J. Lubchenco, and J. M. Mellilo. 1997. Human Domination of Earth's Ecosystems. Science 277:494-499.

Wang, T. H., Y. K. Donaldson, R. P. Brettle, J. E. Bell, and P. Simmonds. 2001. Identification of shared populations of human immunodeficiency virus type 1 infecting microglia and tissue macrophages outside the central nervous system. Journal of Virology 75:1168611699.

Wang, X.-H., R. Aliyari, W.-X. Li, H.-W. Li, K. Kim, R. Carthew, P. Atkinson, and S.-W. Ding. 2006. RNA Interference directs innate immunity against viruses in adult Drosophila. Science 312:452-454.

Warburton, B., and P. Livingstone. 2015. Managing and eradicating wildlife tuberculosis in New Zealand. New Zealand Veterinary Journal 63:77-88.

Ward, D. F., C. Green, R. J. Harris, S. Hartley, P. J. Lester, M. C. Stanley, D. M. Suckling, and R. J. Toft. 2010. Twenty years of Argentine ants in New Zealand: Past research and future priorities for applied management. New Zealand Entomologist 33:68-78.

Wetterer, J. K., X. Espadaler, A. L. Wetterer, D. Aguin-Pombo, and A. M. Franquinho-Aguiar. 2006. Long-term impact of exotic ants on the native ants of Madeira. Ecological Entomology 31:358-368.

Whyard, S., A. D. Singh, and S. Wong. 2009. Ingested double-stranded RNAs can act as species-specific insecticides. Insect Biochemistry and Molecular Biology 39:824-832.

Wilder, S. M., T. R. Barnum, D. A. Holway, A. V. Suarez, and M. D. Eubanks. 2013. Introduced fire ants can exclude native ants from critical mutualist-provided resources. Oecologia 172:197-205.

Wilfert, L., G. Long, H. C. Leggett, P. Schmid-Hempel, R. Butlin, S. J. Martin, and M. Boots. 2016. Deformed wing virus is a recent global epidemic in honeybees driven by Varroa mites. Science 351:594-597.

Williams, T., C. Virto, R. Murillo, and P. Caballero. 2017. Covert infection of insects by baculoviruses. Frontiers in Microbiology 8:1337.

Wilson, E. E., L. M. Mullen, and D. A. Holway. 2009. Life history plasticity magnifies the ecological effects of a social wasp invasion. Proceedings of the National Academy of Sciences 106:12809-12813.

Wilson, E. O. 1971. The insect societies. Harvard University Press. 
Wittkopp, P. J., J. R. True, and S. B. Carroll. 2002. Reciprocal functions of the Drosophila Yellow and Ebony proteins in the development and evolution of pigment patterns. Development 129:1849-1858.

Woolhouse, M. E. J. 2002. Population biology of emerging and re-emerging pathogens. Trends in Microbiology 10:S3-7.

Woolhouse, M. E. J., and C. Dye. 2001. Population biology of emerging and re-emerging pathogens - Preface. Philosophical Transactions of the Royal Society of London. Series B: Biological Sciences 356:981-982.

Woolhouse, M. E. J., D. T. Haydon, and R. Antia. 2005. Emerging pathogens: The epidemiology and evolution of species jumps. Trends in Ecology \& Evolution 20:238244.

Wu, J. Y., M. D. Smart, C. M. Anelli, and W. S. Sheppard. 2012. Honey bees (Apis mellifera) reared in brood combs containing high levels of pesticide residues exhibit increased susceptibility to Nosema (Microsporidia) infection. Journal of Invertebrate Pathology 109:326-329.

Yañez, O., N. Piot, A. Dalmon, J. R. de Miranda, P. Chantawannakul, D. Panziera, E. Amiri, G. Smagghe, D. Schroeder, and N. Chejanovsky. 2020. Bee Viruses: Routes of infection in hymenoptera. Frontiers in Microbiology 11:943.

Yang, L., Y. Tian, Y.-Y. Peng, J. Niu, and J.-J. Wang. 2020. Expression dynamics of core RNAi machinery genes in pea aphids upon exposure to artificially synthesized dsRNA and miRNAs. Insects 11:70.

Yang, X., and D. L. Cox-Foster. 2005. Impact of an ectoparasite on the immunity and pathology of an invertebrate: Evidence for host immunosuppression and viral amplification. Proceedings of the National Academy of Sciences 102:7470-7475.

You, L., F. Zhang, S. Huang, A. Merchant, X. Zhou, and Z. Li. 2020. Over-expression of RNA interference (RNAi) core machinery improves susceptibility to RNAi in silkworm larvae. Insect Molecular Biology 29:353-362.

Yue, C., and E. Genersch. 2005. RT-PCR analysis of deformed wing virus in honeybees (Apis mellifera) and mites (Varroa destructor). Journal of General Virology 86:3419-3424.

Zambon, R. A., V. N. Vakharia, and L. P. Wu. 2006. RNAi is an antiviral immune response against a dsRNA virus in Drosophila melanogaster. Cellular Microbiology 8:880-889.

Zanni, V., D. A. Galbraith, D. Annoscia, C. M. Grozinger, and F. Nazzi. 2017. Transcriptional signatures of parasitization and markers of colony decline in Varroa-infested honey bees (Apis mellifera). Insect Biochemistry and Molecular Biology 87:1-13. 
Zheng, X., S. Li, Y. Si, J. Hu, and Y. Xia. 2020. Locust can detect $\beta$-1, 3-glucan of the fungal pathogen before penetration and defend infection via the Toll signaling pathway. Developmental \& Comparative Immunology 106:103636. 


\section{Supplemental materials}

Supplemental figures and tables Chapter 2:

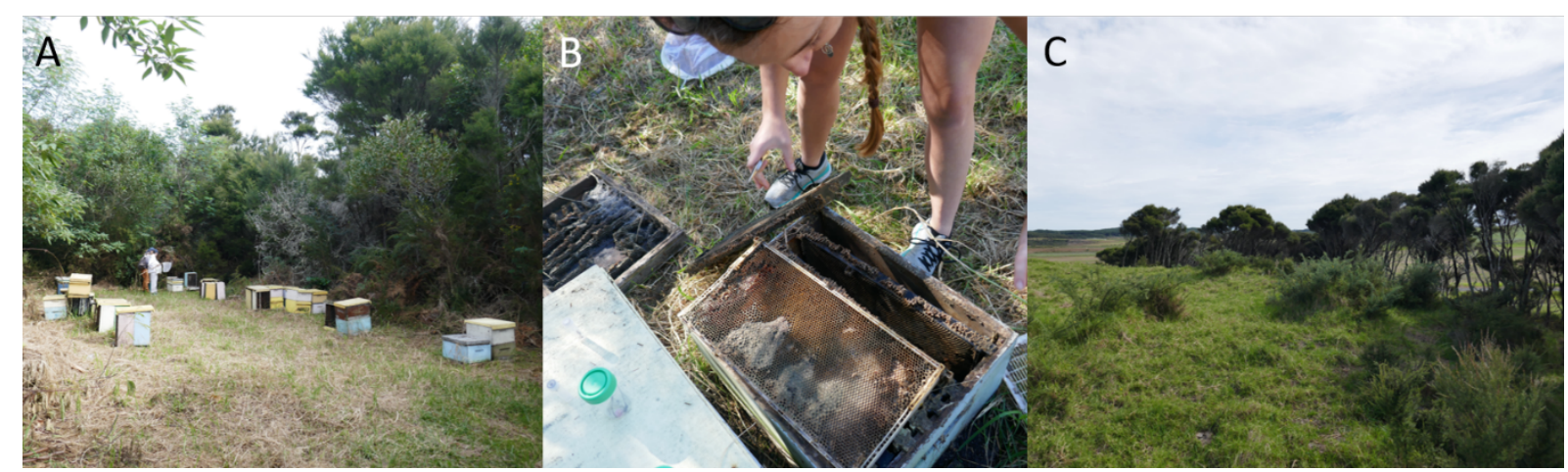

Figure S2.1: Sampling pollinators and associated arthropods in Northland in apiary- and nonapiary sites. A: An apiary site with an ant incursion. B: Sampling Argentine ants, cockroaches and spiders from an abandoned beehive. C: A non-apiary site with kānuka (Kunzea ericoides) trees. Photos by Jana Dobelmann 


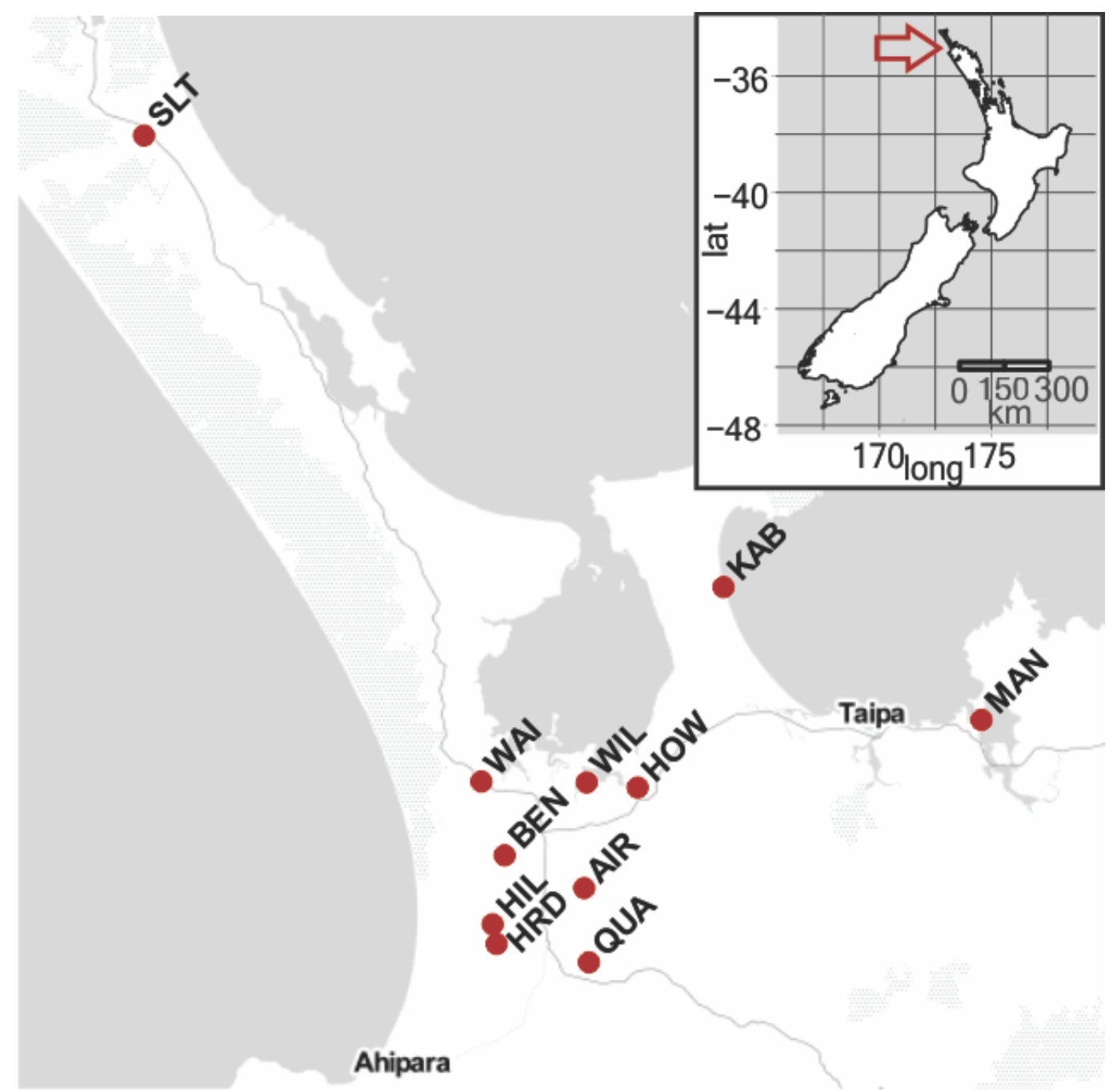

Figure S2.2: Sampling sites in the far north region near Ahipara, Northland, New Zealand. Overview map of New Zealand in the top right corner with red arrow indicating the zoom in on the region shown in the background. AIR, BEN, WAI are sampling locations in apiaries; WAI and SLT had Argentine ant nests; all other sites without apiaries or Argentine ant nests nearby. QUA:-35.11665, 173.28511; AIR: -35.07769, 173.28212; BEN: -35.06049, 173.23116; WAI: -35.02177, 173.21626; SLT: $-34.68195,173.00017$; KAB: -34.91964 , 173.37140; HRD: -35.106945, 173.22600; MAN: -34.98943, 173.53658; HOW: -35.02490, 173.31634; WIL: -35.02227, 173.28375; HIL: -35.09669, 173.22350. 


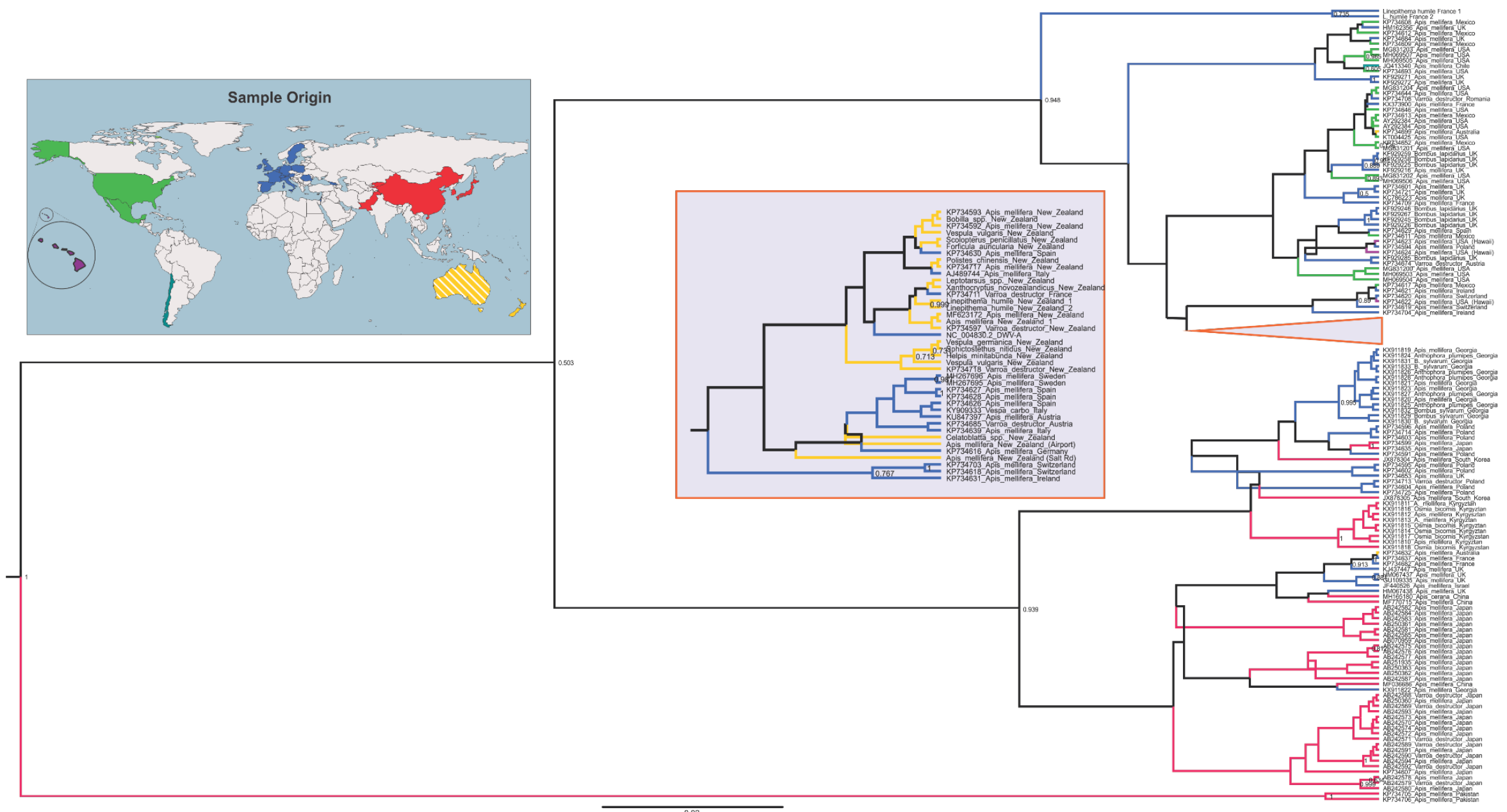

Figure S2.3: Non-dated maximum clade credibility tree for the $R d R p$ fragment (440 bp) of deformed wing virus (DWV) without tip dates. Grey insert with orange frame shows collapsed part of the tree that among others includes the 19 samples from New Zealand from this study. Species name, country of origin and if applicable GenBank accession number are given in the branch label. The branches are coloured according to the lineages' inferred geographic origin as shown on the world map. Posterior support $>0.5$ is given and the scale bar shows substitutions. 


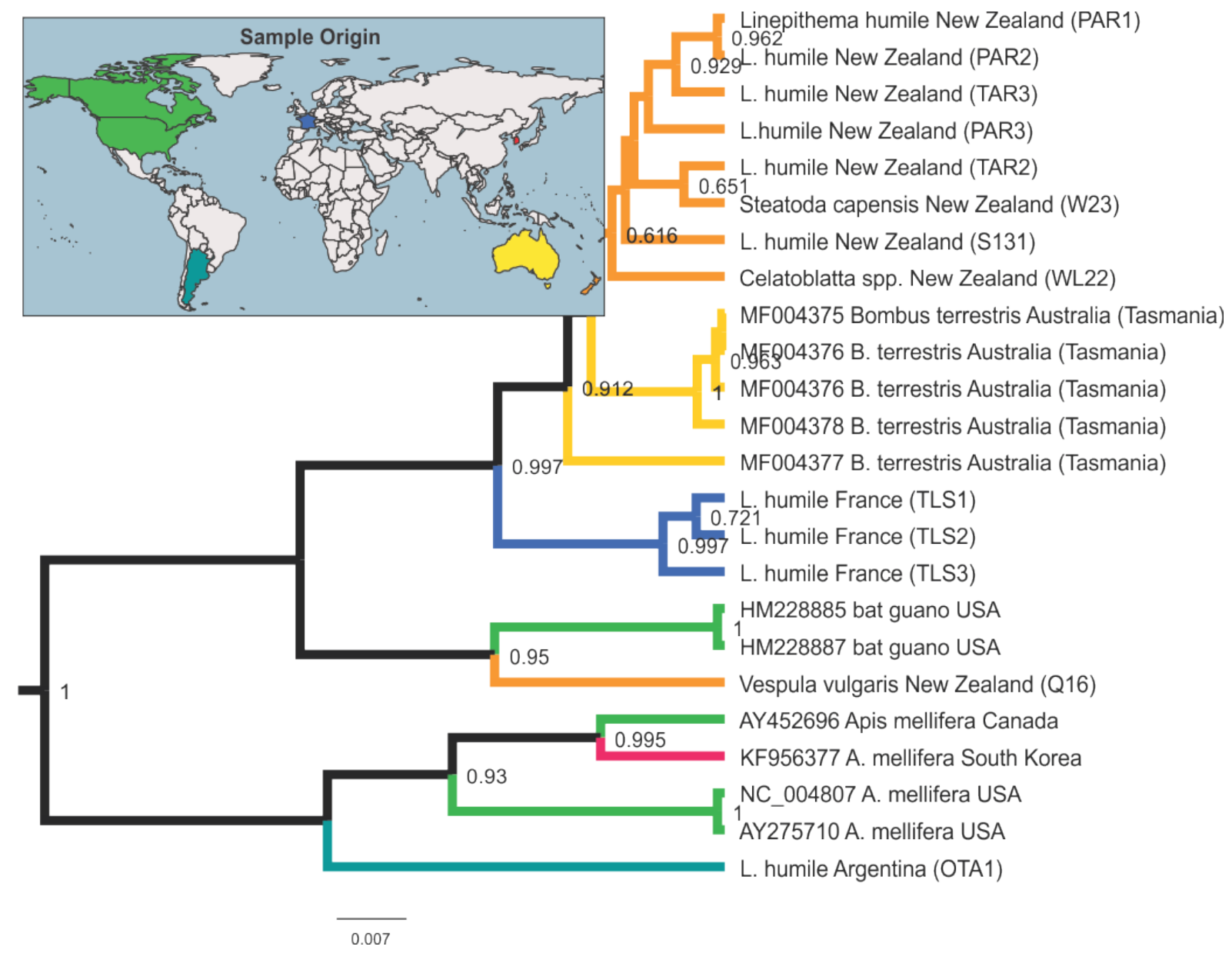

Figure S2.4: Non-dated maximum clade credibility tree for the $v p 3$ fragment (360 bp) of Kashmir bee virus (KBV) without tip dates. Species name, country of origin and if applicable GenBank accession number are given in the branch label. The branches are coloured according to the lineages' inferred geographic origin as shown on the world map. Posterior support $>0.5$ is given and the scale bar shows substitutions. 
Table S2.1: Primers used for virus detection. Screened viruses are deformed wing virus (DWV), black queen cell virus (BQCV), Kashmir bee virus (KBV), Linepithema humile bunya-like virus 1 (LhuBLV1) and Moku virus (MKV). Primers were used in presence/absence PCR and sequencing (+/-) or in strand specific RT-PCR (Repl) as shown in M (Method) column. Forward primer (F) and reverse primer (R), tagged primer (tag-F/tag-R) are given in $3^{\prime}$ to 5 ' direction, with the length of the PCR product in base pairs (pb) and the annealing temperature used in PCR reactions (T). References for primer sequences: DWV (Yue and Genersch 2005), BCQV and KBV (Benjeddou et al. 2001, Singh et al. 2010), LhuBLV1 (Viljakainen et al. 2018), MKV (Garigliany et al. 2017).

\begin{tabular}{|c|c|c|c|c|c|}
\hline Virus & $\begin{array}{l}\text { Target } \\
\text { gene }\end{array}$ & M & Primer sequence & bp & $\begin{array}{l}\mathrm{T} \\
{\left[{ }^{\circ} \mathrm{C}\right]}\end{array}$ \\
\hline DWV & RdRP & $\begin{array}{l}+/- \\
\text { Repl. }\end{array}$ & $\begin{array}{l}\text { F: TCCATCAGGTTCTCCAATAACGGA } \\
\text { R: CCACCCAAATGCTAACTCTAAGCG } \\
\text { tag-F: } \\
\text { gcctgcgcaccgtggTCCATCAGGTTCTCCAATAACGGA }\end{array}$ & 451 & 55 \\
\hline BQCV & $\begin{array}{l}\text { Capsid/ } \\
\text { 3'UTR }\end{array}$ & $+/-$ & $\begin{array}{l}\text { F:TGGTCAGCTCCCACTACCTTAAAC } \\
\text { R:GCAACAAGAAGAAACGTAAACCAC }\end{array}$ & 700 & 56 \\
\hline KBV & Capsid & $\begin{array}{l}+/- \\
\text { Repl. }\end{array}$ & $\begin{array}{l}\text { F: TGTTTGTGGCAATCCAGCTA } \\
\text { R: TACGTCTTCTGCCCATTTCC } \\
\text { Tag-F: } \\
\text { agcetgcgcaccgtggTGTTTGTGGCAATCCAGCTA }\end{array}$ & 625 & 54 \\
\hline LhuBLV1 & RdPR & $\begin{array}{l}+/- \\
\text { Repl. }\end{array}$ & $\begin{array}{l}\text { F:GTCACAGAGGAGCAGATACCA } \\
\text { R:GCCCATAAACCTTGCTCTGC } \\
\text { Tag-R: } \\
\text { agcetgcgcaccgtggGCCCATAAACCTTGCTCTGC }\end{array}$ & 89 & 54 \\
\hline MKV & $\begin{array}{l}\text { Poly } \\
\text { prot. }\end{array}$ & Repl. & $\begin{array}{l}\text { F: CTACAACGCACGCGAGTAGA } \\
\text { R:CCTTTCAAAGCAACGCTACC } \\
\text { Tag-F: } \\
\text { agcetgcgcaccgtggCTACAACGCACGCGAGTAGA }\end{array}$ & 102 & 55 \\
\hline
\end{tabular}

Table S2.2: Sampling location of additional Argentine ant samples used for phylogenetic analyses from Felden et al. (2019).

\begin{tabular}{llll}
\hline $\begin{array}{l}\text { Country } \\
\text {-Location }\end{array}$ & Latitude & Longitude & Collection date \\
\hline \hline $\begin{array}{l}\text { France } \\
\text {-Toulouse }\end{array}$ & 43.5593 & 1.4723 & August 2016 \\
\hline $\begin{array}{l}\text { Argentina } \\
\text {-Otamendi }\end{array}$ & -34.2224 & -58.8961 & January 2015 \\
\hline $\begin{array}{l}\text { New Zealand } \\
\text {-Oakura }\end{array}$ & -39.1195 & 173.9397 & February 2016 \\
-Paraparaumu & -40.963439 & 174.973731 & February 2016 \\
\hline \hline
\end{tabular}


Table S2.3: Trait-tip association of non-dated deformed wing virus (DWV) phylogeny, as calculated with 100 random trees (replicates) in BaTS (Parker et al. 2008). Low parsimony score (PS) represents fewer state changes on the tree and a stronger trait-tip association. Maximum clade (MC) scores show the maximum size of a clade for a trait state, a high MC is positively correlated with trait-tip association.

\begin{tabular}{|c|c|c|c|c|c|c|c|c|c|}
\hline Statistic & $\begin{array}{l}\text { Observed } \\
\text { /expected }\end{array}$ & $\mathrm{n}$ & $\begin{array}{l}\text { observed } \\
\text { mean }\end{array}$ & $\begin{array}{l}\text { lower } \\
95 \% \\
\text { CI }\end{array}$ & $\begin{array}{l}\text { upper } \\
95 \% \\
\text { CI }\end{array}$ & $\begin{array}{l}\text { null } \\
\text { mean }\end{array}$ & $\begin{array}{l}\text { lower } \\
95 \% \\
\text { CI }\end{array}$ & $\begin{array}{l}\text { upper } \\
95 \% \\
\text { CI }\end{array}$ & $\begin{array}{l}p \text { - } \\
\text { value }\end{array}$ \\
\hline \multicolumn{10}{|c|}{ Association Index (AI) } \\
\hline Geographic origin & 0.26 & & 3.82 & 2.81 & 4.84 & 14.80 & 13.86 & 15.68 & $<0.01$ \\
\hline Host species & 0.59 & & 5.82 & 4.72 & 6.89 & 9.87 & 9.22 & 10.44 & $<0.01$ \\
\hline \multicolumn{10}{|c|}{ Parsimony Score (PS) } \\
\hline Geographic origin & 0.36 & & 31.43 & 28.00 & 35.00 & 88.49 & 85.33 & 91.86 & $<0.01$ \\
\hline Host species & 0.72 & & 37.07 & 34.00 & 40.00 & 51.26 & 50.05 & 52.07 & $<0.01$ \\
\hline \multicolumn{10}{|c|}{ Maximum Clade (MC) scores } \\
\hline Apis & & 126 & 14.72 & 9.00 & 24.00 & 7.48 & 5.93 & 9.63 & 0.02 \\
\hline Varroa destructor & & 13 & 1.67 & 1.00 & 3.00 & 1.20 & 1.02 & 1.49 & 0.03 \\
\hline Associate & & 6 & 1.31 & 1.00 & 2.00 & 1.05 & 1.00 & 1.16 & 1 \\
\hline Non-Apis bee & & 23 & 4.23 & 4.00 & 6.00 & 1.66 & 1.31 & 2.05 & $<0.01$ \\
\hline Vespidae & & 5 & 1.07 & 1.00 & 2.00 & 1.04 & 1.00 & 1.15 & 1 \\
\hline Formicidae & & 4 & 2.00 & 2.00 & 2.00 & 1.01 & 1.00 & 1.05 & $<0.01$ \\
\hline Other Hymenoptera & & 2 & 1.00 & 1.00 & 1.00 & 1.01 & 1.00 & 1.01 & 1 \\
\hline Asia & & 50 & 21.52 & 13.00 & 34 & 2.58 & 2.23 & 3.28 & $<0.01$ \\
\hline Europe & & 76 & 14.79 & 14.00 & 19.00 & 3.63 & 3.03 & 4.44 & $<0.01$ \\
\hline North America & & 24 & 3.80 & 3.00 & 6.00 & 1.70 & 1.31 & 2.08 & $<0.01$ \\
\hline Oceania & & 25 & 6.09 & 4.00 & 10.00 & 1.85 & 1.49 & 2.50 & $<0.01$ \\
\hline South America & & 1 & 1.00 & 1.00 & 1.00 & 1.00 & 1.00 & 1.00 & 1 \\
\hline Hawaii & & 3 & 1.05 & 1.00 & 2.00 & 1.03 & 1.00 & 1.08 & 1 \\
\hline
\end{tabular}


Table S2.4: Trait-tip association of non-dated Kashmir bee virus (KBV) phylogeny, as calculated with 100 random trees (replicates) in BaTS (Parker et al. 2008). Low parsimony score (PS) represents fewer state changes on the tree and a stronger trait-tip association. Maximum clade (MC) scores show the maximum size of a clade for a trait state, a high MC is positively correlated with trait-tip association

\begin{tabular}{|c|c|c|c|c|c|c|c|c|c|}
\hline Statistic & $\begin{array}{l}\text { Observed } \\
\text { /expected }\end{array}$ & $\mathrm{n}$ & $\begin{array}{l}\text { observed } \\
\text { mean }\end{array}$ & $\begin{array}{l}\text { lower } \\
95 \% \\
\text { CI }\end{array}$ & $\begin{array}{l}\text { upper } \\
95 \% \\
\text { CI }\end{array}$ & $\begin{array}{l}\text { null } \\
\text { mean }\end{array}$ & $\begin{array}{l}\text { lower } \\
95 \% \\
\text { CI }\end{array}$ & $\begin{array}{l}\text { upper } \\
95 \% \\
\text { CI }\end{array}$ & $\begin{array}{l}p \text { - } \\
\text { value }\end{array}$ \\
\hline
\end{tabular}

\begin{tabular}{|c|c|c|c|c|c|c|c|c|c|}
\hline \multicolumn{10}{|c|}{ Association Index (AI) } \\
\hline Geographic origin & 0.18 & & 0.40 & 0.33 & 0.62 & 2.22 & 1.74 & 2.64 & $<0.01$ \\
\hline Host species & 0.20 & & 0.42 & 0.13 & 0.66 & 2.08 & 1.47 & 2.56 & $<0.01$ \\
\hline \multicolumn{10}{|c|}{ Parsimony Score (PS) } \\
\hline Geographic origin & 0.46 & & 6.26 & 6.00 & 7.00 & 13.55 & 12.01 & 14.90 & $<0.01$ \\
\hline Host species & 0.53 & & 6.61 & 6.00 & 7.00 & 12.43 & 10.78 & 13.79 & $<0.01$ \\
\hline \multicolumn{10}{|c|}{ Maximum Clade (MC) scores } \\
\hline Apis mellifera & & 6 & 3.86 & 2.00 & 4.00 & 1.37 & 1.00 & 2.17 & $<0.01$ \\
\hline other & & 5 & 2.95 & 2.00 & 3.00 & 1.26 & 1.00 & 2.00 & $<0.01$ \\
\hline Linepithema humile & & 10 & 3.50 & 3.00 & 4.00 & 2.01 & 1.10 & 3.06 & 0.09 \\
\hline Bombus & & 3 & 2.95 & 3.00 & 3.00 & 1.09 & 1.00 & 1.72 & $<0.01$ \\
\hline North America & & 4 & 2.00 & 2.00 & 2.00 & 1.34 & 1.00 & 2.00 & 0.15 \\
\hline New Zealand & & 9 & 7.12 & 4.00 & 8.00 & 1.99 & 1.05 & 3.10 & $<0.01$ \\
\hline South America & & 1 & 1.00 & 1.00 & 1.00 & 1.00 & 1.00 & 1.00 & 1.00 \\
\hline Europe & & 3 & 3.00 & 3.00 & 3.00 & 1.10 & 1.00 & 2.00 & $<0.01$ \\
\hline Asia & & 1 & 1.00 & 1.00 & 1.00 & 1.00 & 1.00 & 1.00 & 1.00 \\
\hline Australia & & 5 & 4.35 & 4.00 & 5.00 & 1.27 & 1.00 & 2.00 & $<0.01$ \\
\hline
\end{tabular}




\section{Supplemental figures and tables Chapter 3:}

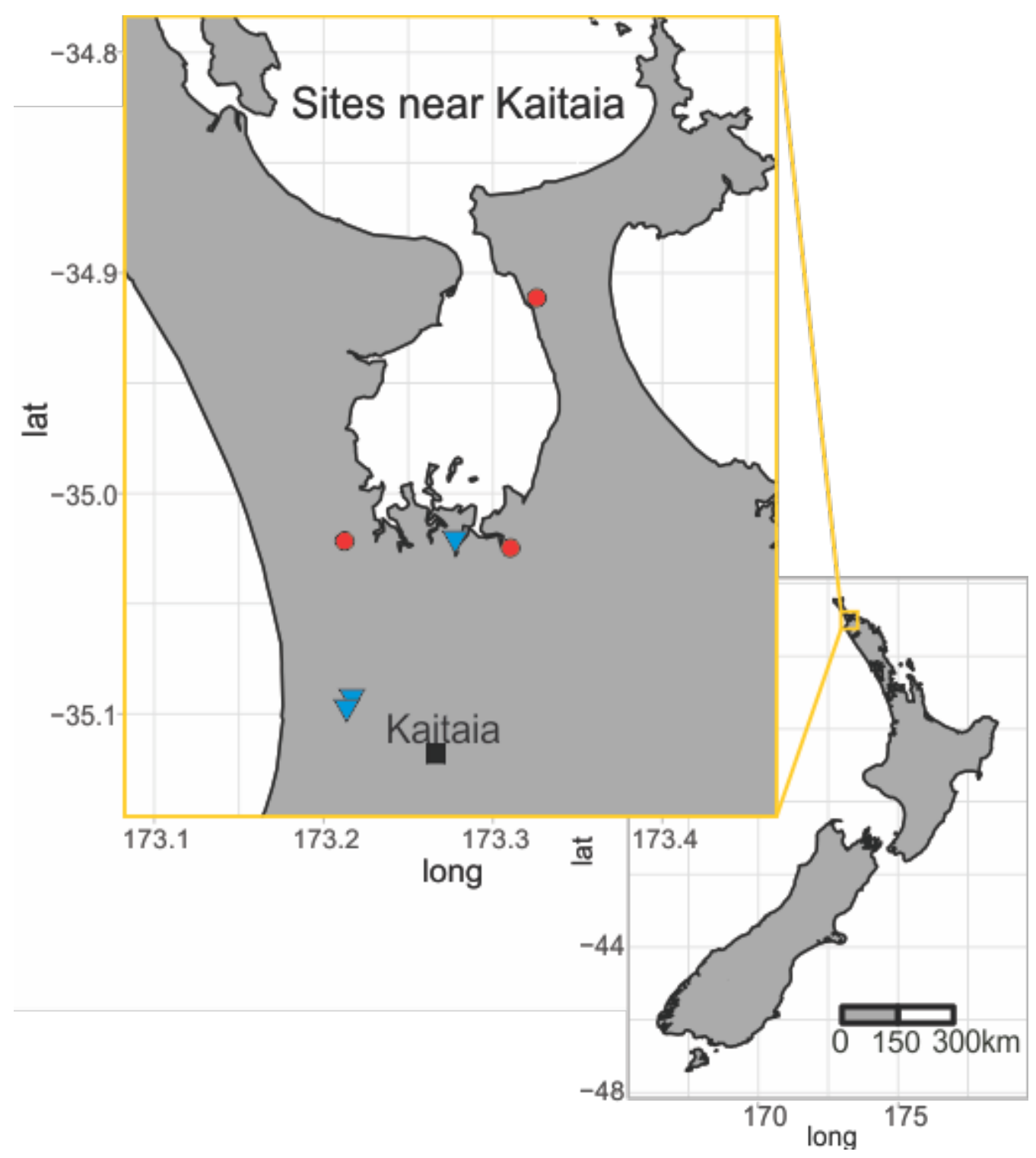

Figure S3.1: Beehive sampling locations for longitudinal study in Northland in New Zealand. Three beehives were placed in each sampling site in January 2019 and samples were collected in monthly intervals until August 2019. Sampling sites without ants are represented by blue triangles (left to right: HLT1, HLT2 and HOW) and sites with Argentine ants by red circles (left to right WAI, WIL, RAN). Bottom right: overview map of New Zealand with a yellow rectangle marking the focus region. 


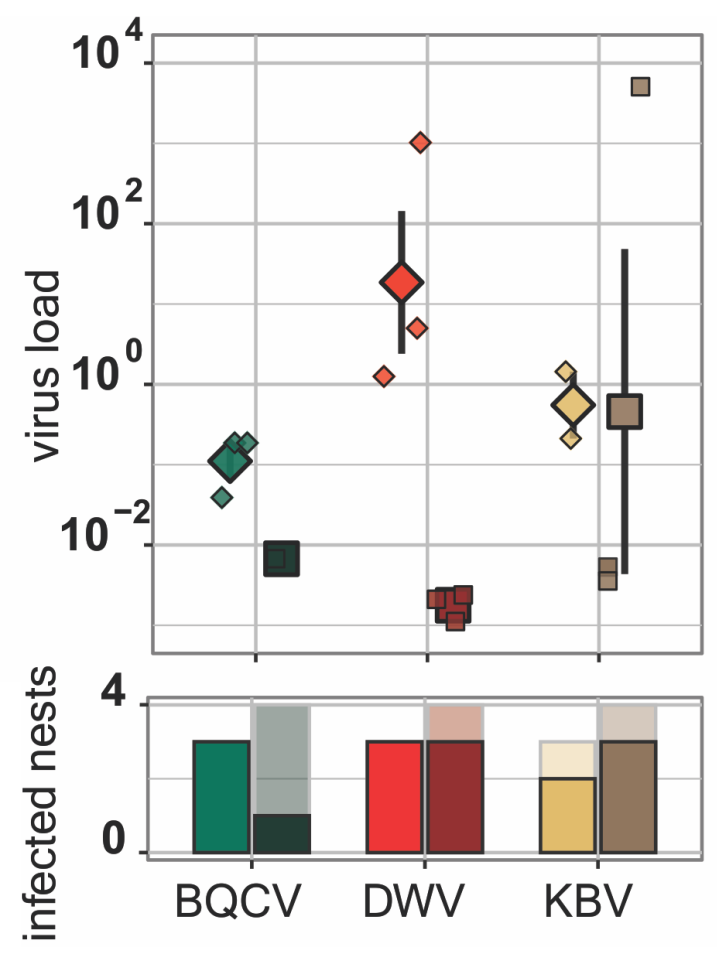

Figure S3.2: Bee-infecting viruses in Argentine ant (L. humile) nests in apiaries and outside apiaries in March. Top: Virus load of ants exposed to bees (diamonds) and without bees (squares). Large symbols indicate the mean \pm standard error and small symbols indicate individual data points. Bottom: Bars indicate the number of infected ant nests with the number of tested ant nests represented by the transparent bar in the background. BQCV: black queen cell virus, DWV: deformed wing virus, KBV: Kashmir bee virus. 

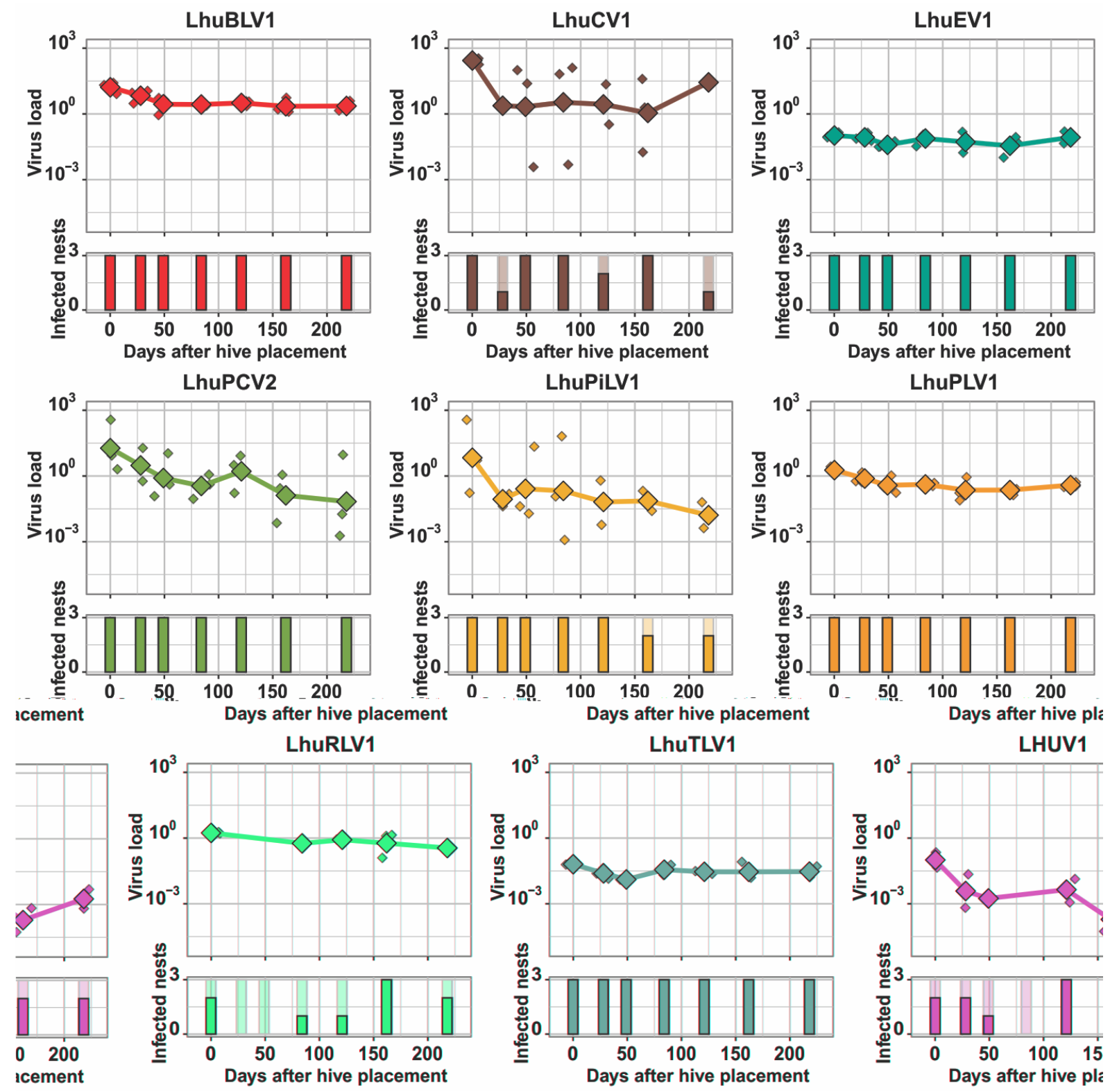

Figure S3.3: Viral load and prevalence of ant-associated viruses in Argentine ant (L. humile) nests after beehives were placed next to the nests. Top: Mean viral load over time is indicated by large filled diamonds, with individual data points shown by small transparent diamonds. Bottom: bars indicate the number of nests that tested positive for the virus. LhuBLV1: Linepithema humile bunya-like virus 1, LhuCV1: Linepithema humile C-virus 1, LhuEV1: Linepithema humile entomopoxvirus 1, LhuPCV2: Linepithema humile polycipivirus 2, LhuPiLV1: Linepithema humile picorna-like virus 1, LhuPLV1: Linepithema humile partitilike virus 1, LhuRLV1: Linepithema humile rhabdo-like virus 1, LhuTLV1: Linepithema humile toti-like virus 1, LHUV1: Linepithema humile virus 1. 
Table S3.1: Primer sequences used in this study. Primes used in TaqMan assays show GenBank accession number used to design primers. Virus acronyms: deformed wing virus (DWV), Kashmir bee virus (KBV), black queen cell virus (BQCV), acute bee paralysis virus (ABPV), Linepithema humile virus 1 (LHUV1), Linepithema humile C virus 1 (LhuCV1), Linepithema humile rhabdo-like virus 1 (LhuRLV1), Linepithema humile bunya-like virus 1 (LhuBLV1), Linepithema humile entomopoxvirus 1 (LhuEV1), Linepithema humile partiti-like virus 1 (LhuPLV1), Linepithema humile polycipivirus 1 (LhuPcV1), Linepithema humile polycipivirus 2 (LhuPcV2), Linepithema humile picorna-like virus 1 (LhuPiLV1), Linepithema humile qinvirus-like virus 1 (LhuQLV1), Linepithema humile toti-like virus 1 (LhuTLV1).

\begin{tabular}{|c|c|c|c|}
\hline \multicolumn{2}{|l|}{$\begin{array}{l}\text { Name } \\
\text { (GenBank accession) }\end{array}$} & Primer sequence & Reference \\
\hline \multicolumn{4}{|c|}{ A. mellifera-SYBR green } \\
\hline \multirow[t]{2}{*}{ DWV } & For. & CGAAACCAACTTCTGAGGAA & \multirow{2}{*}{$\begin{array}{l}\text { (DeGrandi- } \\
\text { Hoffman et al. } \\
\text { 2010) }\end{array}$} \\
\hline & Rev. & GTGTTGATCCCTGAGGCTTA & \\
\hline \multirow[t]{2}{*}{ KBV } & For. & ACCAGGAAGTATTCCCATGGTAAG & \multirow{2}{*}{ This study } \\
\hline & Rev. & TGGAGCTATGGTTCCGTTCAG & \\
\hline \multirow[t]{2}{*}{ BQCV } & For. & TCCTCAAATCTGGAGCGAAC & \multirow{2}{*}{$\begin{array}{l}\text { (Runckel et al. } \\
\text { 2011) }\end{array}$} \\
\hline & Rev. & GTATTCGCTGGCCGTAAAAC & \\
\hline \multirow[t]{2}{*}{ Nosema ceranae } & For. & CGGATAAAAGAGTCCGTTACC & \multirow{2}{*}{$\begin{array}{l}\text { (Chen et al. } \\
\text { 2008) }\end{array}$} \\
\hline & Rev. & TGAGCAGGGTTCTAGGGAT & \\
\hline \multirow[t]{2}{*}{ Lotmaria passim } & For. & AGTATGAGCAGTAGGTTTTATTATA & \multirow{2}{*}{$\begin{array}{l}\text { (Vejnovic et al. } \\
\text { 2018) }\end{array}$} \\
\hline & Rev. & GCCAAACACCAATAACTGGTACT & \\
\hline \multirow[t]{2}{*}{$\beta$-actin } & For. & TGCCAACACTGTCCTTTCTG & \multirow{2}{*}{$\begin{array}{l}\text { (Mutti et al. } \\
\text { 2011) }\end{array}$} \\
\hline & Rev. & AGAATTGACCCACCAATCCA & \\
\hline \multicolumn{4}{|l|}{ L. humile - TaqMan } \\
\hline \multirow{3}{*}{$\begin{array}{l}\text { ABPV } \\
\left(\mathrm{NC} \_002548\right)\end{array}$} & For. & GGTTATGCTCAGGAGAGATTCCTAAA & \multirow{24}{*}{ This study } \\
\hline & Rev. & TCCAACACAACAAGCCTTAATACTG & \\
\hline & Pr. & ACACAACATGGTTACCCATA & \\
\hline \multirow{3}{*}{$\begin{array}{l}\text { BQCV } \\
\left(\mathrm{NC} \_003784\right)\end{array}$} & For. & GGTGCGGGAGATGATATGGA & \\
\hline & Rev. & GCCGTCTGAGATGCATGAATAC & \\
\hline & Pr. & TTTCCATCTTTATCGGTACGCCGCC & \\
\hline \multirow{3}{*}{$\begin{array}{l}\text { DWV } \\
\text { (NC_004830) }\end{array}$} & For. & AAATACTGTAAAGTGGCGGACGTT & \\
\hline & Rev. & CGTTCCTTCTACCGAGACCTTGT & \\
\hline & Pr. & TAAAACATCCAACTAGACCTGT & \\
\hline \multirow{3}{*}{$\begin{array}{l}\text { KBV } \\
\left(\mathrm{NC} \_004807\right)\end{array}$} & For. & GCATCGAGCGCATTCCA & \\
\hline & Rev. & TACACCTCCTACAATATCAGCAAACC & \\
\hline & Pr. & TGCAAAGCCGGTAACT & \\
\hline \multirow{3}{*}{$\begin{array}{l}\text { LHUV1 } \\
\text { (MF417634) }\end{array}$} & For. & AACAGTATGCAGAGACAAGATTCGA & \\
\hline & Rev. & ATTCAGCTGGAGTACTTGCATGTTA & \\
\hline & Pr. & CCGGACGCACACAA & \\
\hline \multirow{3}{*}{$\begin{array}{l}\text { LhuCV1 } \\
\text { (MH213244) }\end{array}$} & For. & GGGAGATGGGCTGGAAGTG & \\
\hline & Rev. & TTGGCTCTCGACACCATCAG & \\
\hline & Pr. & CAGCTGTCGCCCTT & \\
\hline \multirow{3}{*}{$\begin{array}{l}\text { LhuRLV1 } \\
(\mathrm{MH} 213246)\end{array}$} & For. & TGGAACCCAAGAGCATGGAT & \\
\hline & Rev. & TCTTTGGTACCCCAATTGGATCT & \\
\hline & Pr. & CCTCCCAGATTGTGC & \\
\hline \multirow{3}{*}{$\begin{array}{l}\text { LhuBLV1 } \\
\text { (MH213237) }\end{array}$} & For. & AAGGTGAATGTTTGTCTGAACTACCA & \\
\hline & Rev. & AAGACAAGACAGAAGAAGATGCATTG & \\
\hline & Pr. & ATCTCCAACATTGATATAACGTCTA & \\
\hline
\end{tabular}




\begin{tabular}{|c|c|c|}
\hline LhuEV1 & For. & TGCAAGTACCAGAGTAGAGAACGGTAT \\
\hline$(\mathrm{MH} 213250)$ & Rev. & AGATGCTTTAGCCTGTCCAGAGTT \\
\hline & Pr. & TTCGTCGACAATAAAATCCTGAA \\
\hline LhuPLV1 & For. & CCACGTCCACCTCGTCAATT \\
\hline$(\mathrm{MH} 213239-240)$ & Rev. & GTTGAAAGAAAAGCGGGCTTAG \\
\hline & Pr. & ACGCAAAACCTCAGGTG \\
\hline LhuPcV1 & For. & TTTTTTGGCACATCGAACCA \\
\hline (MH213247) & Rev. & TCAGAGACAGTTCGCTCAATACG \\
\hline & Pr. & CCAGCCTTACTAACGAT \\
\hline LhuPcV2 & For. & CCATGTTCTCGGTGGAGCAT \\
\hline (MH213248) & Rev. & GACACGGGTGGATATAGGCATT \\
\hline & Pr. & CCTCTGCGCAGTTAA \\
\hline LhuPiLV1 & For. & GGATGGATTCACGTCGTTCAA \\
\hline$(\mathrm{MH} 213235)$ & Rev. & AACCGTCCGGCTTGGAA \\
\hline & Pr. & CAACGTGCGTTCTC \\
\hline LhuQLV1 & For. & TCTTGAGCTTCCTGTGTTAACCAA \\
\hline$(\mathrm{MH} 213241)$ & Rev. & AATGCCGTTTTTGCACCAA \\
\hline & Pr. & TATACCCCAACCACCTAGG \\
\hline LhuTLV1 & For. & GAGCAGCAATGGCGAAGAC \\
\hline (MH213243) & Rev. & TCCTCAAATGCTGTACCGCTATC \\
\hline & Pr. & CCTAGAGGACTATCGCACAA \\
\hline Dynactin subunit 4 & For. & AACTGCGGCTCCTTAAAACCTA \\
\hline (LOC105671957) & Rev. & GGACAGTAATGAGAGTCAACCTCTTG \\
\hline & Pr. & TTGTCGACACTGCTTGAA \\
\hline
\end{tabular}




\section{Supplemental figures and tables Chapter 4:}

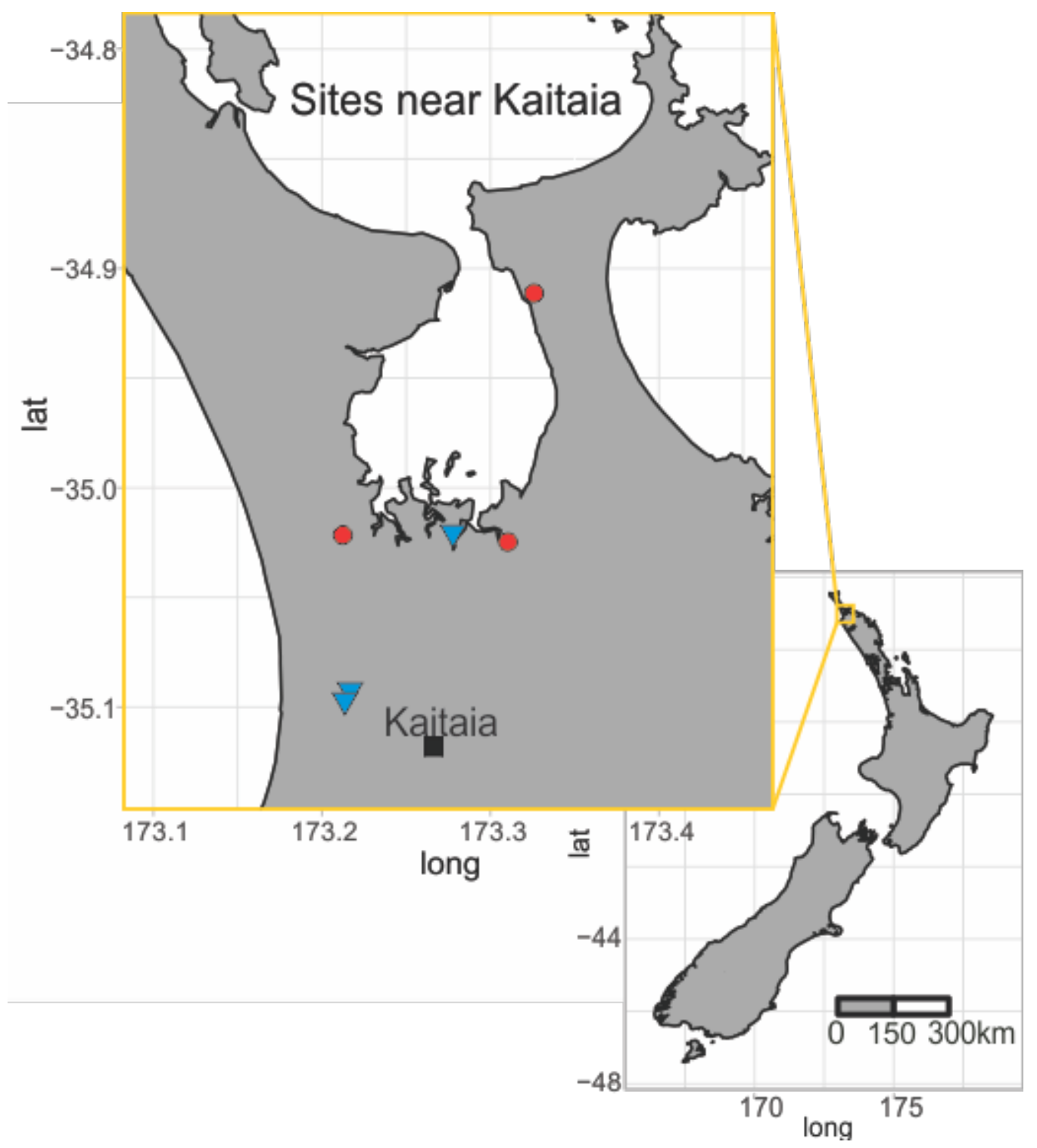

Figure S4.1: Beehive sampling locations in Northland in New Zealand. Top left: Zoom on Kaitaia. Three beehives were placed in each sampling site in January 2019 and samples used for RNA sequencing were collected in June 2019. Sampling sites without ants are represented by blue triangles (left to right: HLT1, HLT2 and HOW) and sites with Argentine ants by red circles (left to right WAI, WIL, RAN). Two hives were sampled and sequenced at each site, except for RAN (top right) where only one sample had sufficient RNA quality for sequencing. Bottom right: overview map of New Zealand with a yellow rectangle marking the focus region. 

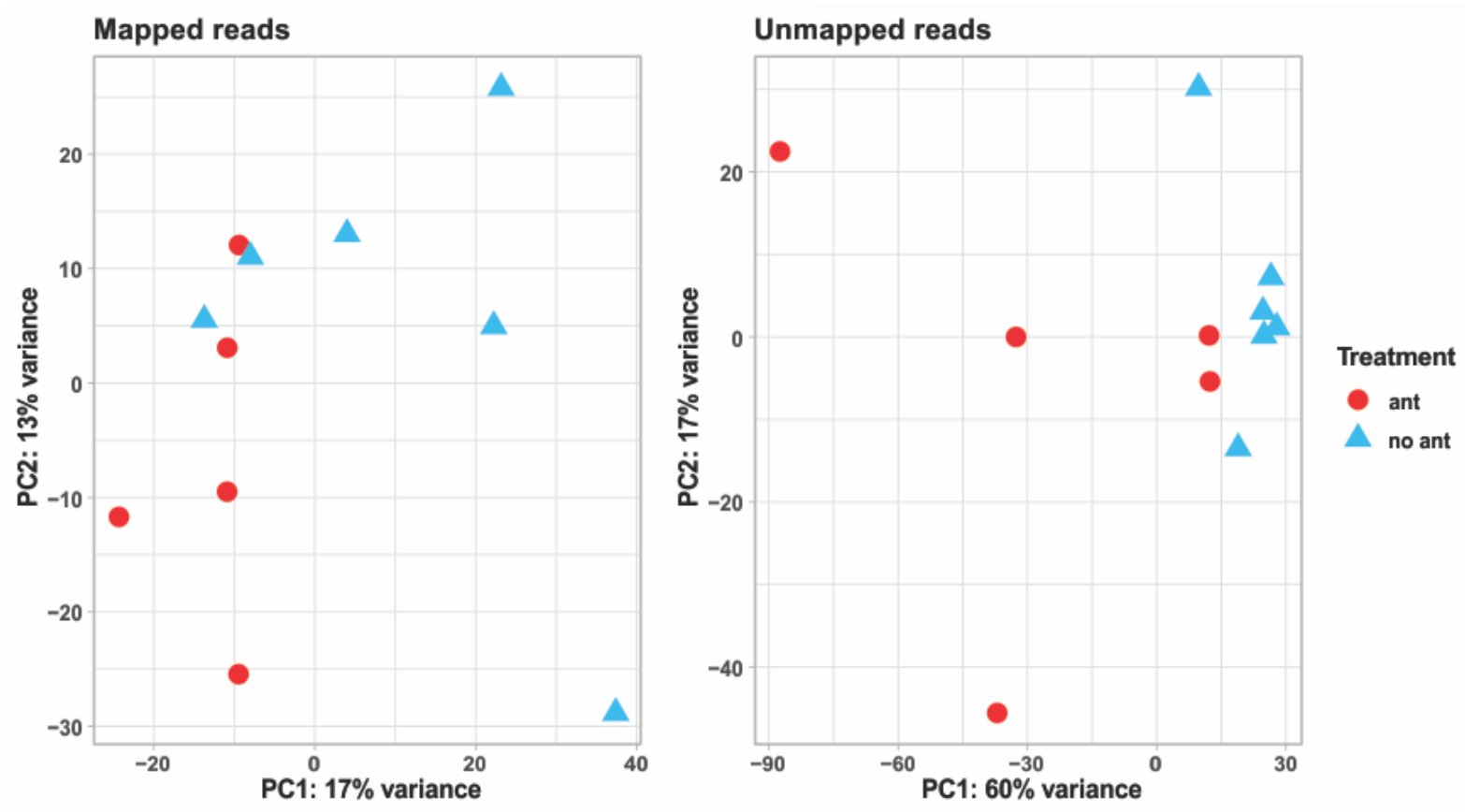

Figure S4.2: Principal component analysis (PCA) of RNA sequencing reads that mapped onto the honey bee genome (left) and reads that did not map concordantly (right). Honey bees were sampled from sites with (red circle) and without (blue triangle) Argentine ants. Mapped reads were used to analyse gene expression in honey bees and unmapped reads were used for the discovery and comparison of viral infections in bees. PCA plots were generated using DESeq 2 (Love et al. 2014) using the 500 most variable genes. The axis labels show how much variation the first and second principal component (PC) account for.

Table S4.1: Sequence library and alignment stats. Reads were mapped onto the honey bee genome (Amel_HAv3.1) in HISAT2 v2.1.0. Mapped reads were used to analyse transcriptomic responses and unmapped reads were used to analyse viral infections in honey bees with and without Argentine ants. Note that the low number of mapped and large number of unmapped reads in hive 8 is likely driven by high DWV titres.

Hive (sample ID) Overall align. rate (\%) Lib. size mapped Lib. size unmapped

\begin{tabular}{llll}
\hline \hline 2 & 90.44 & $51,046,110$ & $2,595,017$ \\
\hline 4 & 95.38 & $50,514,223$ & $1,182,029$ \\
\hline 5 & 95.10 & $55,137,324$ & $1,142,937$ \\
\hline 8 & 84.44 & $44,904,071$ & $4,306,239$ \\
\hline 9 & 95.29 & $55,658,004$ & $1,263,090$ \\
\hline 10 & 95.68 & $50,036,011$ & $1,036,586$ \\
\hline 12 & 96.28 & $61,736,274$ & $1,004,776$ \\
\hline 13 & 95.15 & $53,368,136$ & $1,311,457$ \\
\hline 16 & 95.47 & $52,383,210$ & $1,198,263$ \\
\hline 17 & 95.03 & $54,526,080$ & $1,351,785$ \\
\hline \hline
\end{tabular}


Table S4.2: Results from BLASTx searches of de novo assembled transcripts against the NCBI virus database (viral.nonredundant_protein.1.protein.faa). Searches were run with an $E$-value cut-off of $10^{-5}$ and all hits with $<85 \%$ identity and $<300$ base pairs were filtered out. $*$ The contig that matched Hubei picorna-like virus 15 was more closely related to Gorebridge virus (MH614301) in a manual BLASTn search against all nucleotide sequences on GenBank.

\begin{tabular}{lllllll}
$\begin{array}{l}\text { Reference } \\
\text { sequence ID }\end{array}$ & Organism & \% Ident & Length & $\begin{array}{l}\text { mis- } \\
\text { match }\end{array}$ & $\begin{array}{l}\text { Gap } \\
\text { open }\end{array}$ & $\begin{array}{l}\text { Bit } \\
\text { score }\end{array}$ \\
\hline NP_733846 & Aphid lethal paralysis virus & 90.718 & 808 & 73 & 1 & 1483 \\
\hline YP_053924 & Arabis mosaic virus & 94.753 & 324 & 17 & 0 & 638 \\
\hline NP_620564 & Black queen cell virus & 93.353 & 1655 & 83 & 2 & 3157 \\
\hline NP_853560 & Deformed wing virus & 99.101 & 2893 & 26 & 0 & 5919 \\
\hline YP_009336541 & Hubei picorna-like virus 15* & 86.364 & 506 & 69 & 0 & 902 \\
\hline NP_851403 & Kashmir bee virus & 98.779 & 1229 & 15 & 0 & 2490 \\
\hline YP_009388492 & Lake Sinai virus SA2 & 96.403 & 417 & 15 & 0 & 848 \\
\hline YP_009388501 & Lake Sinai virus TO & 95.873 & 848 & 35 & 0 & 1534 \\
\hline YP_009305421 & Moku virus & 98.874 & 1954 & 22 & 0 & 3915 \\
\hline NP_049374 & Sacbrood virus & 98.531 & 2859 & 41 & 1 & 5642 \\
\hline NP_620765 & Tomato ringspot virus & 98.928 & 1119 & 12 & 0 & 2283 \\
\hline YP 007889822 & White clover cryptic virus 2 & 97.000 & 300 & 9 & 0 & 570 \\
\hline \hline
\end{tabular}


Table S4.3: List of 141 differentially expressed genes between bees with and bees without Argentine ants with adjusted $\mathrm{p}<0.05$ and an absolute fold change $(\mathrm{FC})>2$. Genes are ranked from highest to lowest FC. A positive fold change indicates a higher expression in bees with ants compared to bees without ants and a negative fold change signifies lower expression.

\begin{tabular}{|c|c|c|c|}
\hline Gene ID & Gene description & $\log 2 \mathrm{FC}$ & Adj. $p$-value \\
\hline LOC552206 & putative ATP-dependent RNA helicase me31b & 29.216 & $5.11 \mathrm{E}-18$ \\
\hline MSTRG.132 & & 11.184 & 2.69E-02 \\
\hline Mir6067 & microRNA mir-6067 & 6.513 & $1.15 \mathrm{E}-04$ \\
\hline LOC102655710 & basic proline-rich protein & 6.501 & $2.31 \mathrm{E}-02$ \\
\hline LOC408642 & NAD kinase 2, mitochondrial & 4.218 & $6.08 \mathrm{E}-06$ \\
\hline LOC552685 & pupal cuticle protein & 3.706 & $2.53 \mathrm{E}-02$ \\
\hline LOC113219380 & collagen alpha-1(IV) chain & 3.671 & $5.04 \mathrm{E}-03$ \\
\hline LOC725903 & zinc finger protein 512B & 3.667 & $1.35 \mathrm{E}-02$ \\
\hline LOC100576418 & tetra-peptide repeat homeobox protein 1 & 3.390 & $7.71 \mathrm{E}-03$ \\
\hline Mir3727 & microRNA mir-3727 & 3.189 & $1.88 \mathrm{E}-03$ \\
\hline LOC102654146 & antifreeze protein Maxi & 2.978 & $3.73 \mathrm{E}-02$ \\
\hline LOC725283 & uncharacterized LOC725283 & 2.782 & $5.72 \mathrm{E}-03$ \\
\hline LOC551656 & DNA translocase FtsK-like & 2.718 & $1.35 \mathrm{E}-03$ \\
\hline LOC411609 & pancreatic triacylglycerol lipase & 2.694 & $2.21 \mathrm{E}-03$ \\
\hline LOC724464 & cuticular protein & 2.652 & $8.00 \mathrm{E}-04$ \\
\hline apd-3 & apidermin 3 & 2.444 & $2.01 \mathrm{E}-02$ \\
\hline LOC102654905 & uncharacterized LOC102654905 & 2.391 & $2.49 \mathrm{E}-04$ \\
\hline LOC724382 & cuticular protein analogous to peritrophins 3-A & 2.387 & $1.50 \mathrm{E}-03$ \\
\hline LOC100577043 & uncharacterized LOC100577043 & 2.368 & $6.47 \mathrm{E}-03$ \\
\hline LOC102654686 & uncharacterized LOC102654686 & 2.350 & $3.22 \mathrm{E}-03$ \\
\hline MSTRG.13947 & & 2.191 & $2.01 \mathrm{E}-02$ \\
\hline Y-y & Yellow-y & 2.161 & $2.69 \mathrm{E}-02$ \\
\hline LOC725842 & $\begin{array}{l}\text { elongation of very long chain fatty acids protein 6- } \\
\text { like }\end{array}$ & 2.139 & $3.87 \mathrm{E}-02$ \\
\hline LOC100577976 & GSK3-beta interaction protein & 2.126 & $2.37 \mathrm{E}-02$ \\
\hline CPR27 & cuticular protein 27 & 2.064 & $2.49 \mathrm{E}-04$ \\
\hline LOC100578329 & fatty acyl-CoA reductase wat & 2.050 & $2.67 \mathrm{E}-02$ \\
\hline LOC100577901 & uncharacterized LOC100577901 & 1.977 & $4.41 \mathrm{E}-04$ \\
\hline LOC413113 & probable serine/threonine-protein kinase samkC & 1.895 & $8.61 \mathrm{E}-06$ \\
\hline LOC727145 & apidermin-like & 1.869 & $3.48 \mathrm{E}-02$ \\
\hline LOC410122 & glyceraldehyde-3-phosphate dehydrogenase 2 & 1.788 & $2.09 \mathrm{E}-02$ \\
\hline LOC408365 & uncharacterized LOC408365 & 1.772 & $4.11 \mathrm{E}-04$ \\
\hline Cpap3-c & cuticular protein analogous to peritrophins 3-C & 1.740 & $2.50 \mathrm{E}-09$ \\
\hline LOC100578586 & $\begin{array}{l}\text { mediator of RNA polymerase II transcription } \\
\text { subunit } 15\end{array}$ & 1.730 & $1.46 \mathrm{E}-03$ \\
\hline LOC100576161 & uncharacterized LOC100576161 & 1.594 & $1.14 \mathrm{E}-02$ \\
\hline LOC100576738 & uncharacterized LOC100576738 & 1.561 & $4.87 \mathrm{E}-03$ \\
\hline LOC726900 & proteasome assembly chaperone 2 & 1.561 & $2.09 \mathrm{E}-02$ \\
\hline LOC408970 & flocculation protein FLO11 & 1.556 & $3.52 \mathrm{E}-05$ \\
\hline LOC551323 & uncharacterized LOC551323 & 1.547 & $1.18 \mathrm{E}-03$ \\
\hline LOC100576769 & uncharacterized LOC100576769 & 1.533 & $2.25 \mathrm{E}-02$ \\
\hline LOC552154 & uncharacterized LOC552154 & 1.499 & $2.63 \mathrm{E}-02$ \\
\hline LOC412222 & uncharacterized LOC412222 & 1.469 & $7.91 \mathrm{E}-03$ \\
\hline LOC724951 & 1-acyl-sn-glycerol-3-phosphate acyltransferase beta & 1.430 & $2.31 \mathrm{E}-02$ \\
\hline LOC727165 & flocculation protein FLO11 & 1.427 & $8.61 \mathrm{E}-06$ \\
\hline LOC411983 & putative fatty acyl-CoA reductase & 1.414 & $3.11 \mathrm{E}-02$ \\
\hline LOC551905 & uncharacterized LOC551905 & 1.386 & $4.40 \mathrm{E}-02$ \\
\hline
\end{tabular}




\begin{tabular}{|c|c|c|c|}
\hline LOC725404 & actin, muscle & 1.375 & $1.97 \mathrm{E}-02$ \\
\hline LOC724569 & uncharacterized LOC724569 & 1.369 & $2.25 \mathrm{E}-02$ \\
\hline Obp16 & odorant binding protein 16 & 1.355 & $1.67 \mathrm{E}-02$ \\
\hline LOC100576934 & uncharacterized LOC100576934 & 1.343 & $3.85 \mathrm{E}-03$ \\
\hline LOC551805 & phospholipase B1, membrane-associated & 1.339 & $4.45 \mathrm{E}-02$ \\
\hline LOC100577574 & leucine-rich repeat-containing protein $4 \mathrm{C}$ & 1.309 & $3.83 \mathrm{E}-02$ \\
\hline Cpap3-b & cuticular protein analogous to peritrophins $3-\mathrm{B}$ & 1.293 & $1.20 \mathrm{E}-05$ \\
\hline Pla2 & phospholipase A2 & 1.290 & $1.66 \mathrm{E}-02$ \\
\hline LOC408552 & collagen alpha-1(IV) chain & 1.278 & $4.66 \mathrm{E}-02$ \\
\hline LOC725838 & uncharacterized LOC725838 & 1.265 & $3.33 \mathrm{E}-02$ \\
\hline Apd-2 & apidermin 2 & 1.258 & $4.76 \mathrm{E}-02$ \\
\hline MSTRG.4154 & & 1.256 & $2.31 \mathrm{E}-03$ \\
\hline LOC413605 & cytochrome $\mathrm{cl}$, heme protein, mitochondrial & 1.247 & $4.79 \mathrm{E}-02$ \\
\hline LOC411864 & chaoptin & 1.242 & $4.64 \mathrm{E}-02$ \\
\hline LOC725364 & flightin & 1.237 & $4.86 \mathrm{E}-02$ \\
\hline LOC552724 & zinc finger $\mathrm{CCCH}$ domain-containing protein 13 & 1.204 & $5.34 \mathrm{E}-03$ \\
\hline LOC410913 & $\begin{array}{l}\text { sodium/potassium-transporting ATPase subunit } \\
\text { alpha }\end{array}$ & 1.192 & $1.91 \mathrm{E}-03$ \\
\hline LOC725813 & uncharacterized LOC725813 & 1.103 & $6.35 \mathrm{E}-05$ \\
\hline LOC551369 & actin, clone 205 & 1.096 & $1.18 \mathrm{E}-03$ \\
\hline Cpap3-c & cuticular protein analogous to peritrophins $3-\mathrm{C}$ & 1.089 & $1.14 \mathrm{E}-02$ \\
\hline TpnCIIa & troponin $\mathrm{C}$ type IIa & 1.083 & $1.92 \mathrm{E}-02$ \\
\hline Obp18 & odorant binding protein 18 & 1.048 & $6.97 \mathrm{E}-03$ \\
\hline LOC413679 & cuticular protein analogous to peritrophins 3-E & 1.039 & $1.16 \mathrm{E}-02$ \\
\hline LOC552217 & uncharacterized LOC552217 & 1.033 & $3.19 \mathrm{E}-02$ \\
\hline LOC102654584 & uncharacterized LOC102654584 & -1.101 & $1.30 \mathrm{E}-02$ \\
\hline LOC408780 & $\begin{array}{l}\text { signal-induced proliferation-associated 1-like } \\
\text { protein } 2\end{array}$ & -1.105 & $4.22 \mathrm{E}-02$ \\
\hline LOC107965402 & uncharacterized LOC107965402 & -1.113 & $2.37 \mathrm{E}-02$ \\
\hline LOC727243 & uncharacterized LOC727243 & -1.123 & $7.99 \mathrm{E}-03$ \\
\hline LOC552086 & aldose 1-epimerase & -1.156 & $2.31 \mathrm{E}-02$ \\
\hline LOC725243 & kielin/chordin-like protein & -1.173 & $4.27 \mathrm{E}-02$ \\
\hline LOC551571 & pyrokinin-like receptor 1 & -1.175 & $2.17 \mathrm{E}-02$ \\
\hline LOC100577211 & uncharacterized LOC100577211 & -1.203 & $2.14 \mathrm{E}-02$ \\
\hline LOC100577771 & uncharacterized LOC100577771 & -1.220 & $4.20 \mathrm{E}-02$ \\
\hline LOC113218556 & glutamic acid-rich protein-like & -1.249 & $1.16 \mathrm{E}-02$ \\
\hline LOC725761 & $\begin{array}{l}\text { uncharacterized abhydrolase domain-containing } \\
\text { protein DDB G0269086 }\end{array}$ & -1.287 & 4.88E-02 \\
\hline MSTRG.15383 & & -1.361 & $2.01 \mathrm{E}-03$ \\
\hline LOC113218870 & uncharacterized LOC113218870 & -1.394 & $2.09 \mathrm{E}-02$ \\
\hline MSTRG.2255 & & -1.439 & $2.13 \mathrm{E}-02$ \\
\hline LOC100578051 & uncharacterized LOC100578051 & -1.454 & $4.77 \mathrm{E}-02$ \\
\hline LOC550899 & UPF0769 protein C21 orf59 homolog & -1.456 & $7.71 \mathrm{E}-03$ \\
\hline LOC726487 & histone $\mathrm{H} 2 \mathrm{~B}$ & -1.479 & $1.92 \mathrm{E}-02$ \\
\hline LOC102655676 & uncharacterized LOC102655676 & -1.487 & 4.47E-02 \\
\hline MSTRG.852 & & -1.526 & $2.13 \mathrm{E}-03$ \\
\hline LOC410951 & RNA binding protein fox-1 homolog 2 & -1.536 & $4.98 \mathrm{E}-02$ \\
\hline MSTRG.4140 & & -1.554 & $2.39 \mathrm{E}-02$ \\
\hline LOC409608 & peroxidasin & -1.567 & $2.68 \mathrm{E}-02$ \\
\hline MSTRG.846 & & -1.586 & $3.73 \mathrm{E}-02$ \\
\hline LOC409608 & peroxidasin & -1.589 & $4.72 \mathrm{E}-02$ \\
\hline LOC102655249 & uncharacterized LOC102655249 & -1.639 & $1.92 \mathrm{E}-02$ \\
\hline MSTRG.800 & & -1.661 & $4.51 \mathrm{E}-02$ \\
\hline
\end{tabular}




\begin{tabular}{|c|c|c|c|}
\hline LOC113218641 & uncharacterized LOC113218641 & -1.676 & 4.76E-02 \\
\hline MSTRG.15382 & & -1.679 & $4.61 \mathrm{E}-02$ \\
\hline MSTRG.8329 & & -1.684 & $1.00 \mathrm{E}-02$ \\
\hline LOC411078 & nephrin & -1.694 & $2.38 \mathrm{E}-02$ \\
\hline LOC413215 & lachesin & -1.700 & $1.66 \mathrm{E}-02$ \\
\hline Obp13 & odorant binding protein 13 & -1.723 & $4.37 \mathrm{E}-02$ \\
\hline LOC409608 & peroxidasin & -1.834 & $4.16 \mathrm{E}-02$ \\
\hline LOC726298 & putative transcription factor SOX-15 & -1.852 & $3.61 \mathrm{E}-02$ \\
\hline LOC100577614 & uncharacterized LOC100577614 & -1.861 & $2.84 \mathrm{E}-02$ \\
\hline LOC409608 & peroxidasin & -1.866 & $6.55 \mathrm{E}-03$ \\
\hline LOC413153 & uncharacterized LOC413153 & -1.871 & $2.82 \mathrm{E}-02$ \\
\hline LOC100577453 & RNA-binding protein 25 & -1.887 & $1.18 \mathrm{E}-03$ \\
\hline LOC724830 & uncharacterized LOC724830 & -1.924 & $2.68 \mathrm{E}-02$ \\
\hline LOC410856 & 28 S ribosomal protein S29, mitochondrial & -1.936 & $6.55 \mathrm{E}-03$ \\
\hline MSTRG.850 & & -1.970 & $2.84 \mathrm{E}-02$ \\
\hline NLG-3 & neuroligin 3 & -1.975 & $4.40 \mathrm{E}-02$ \\
\hline LOC409608 & peroxidasin & -2.002 & $4.52 \mathrm{E}-02$ \\
\hline MSTRG.13470 & & -2.012 & $6.55 \mathrm{E}-03$ \\
\hline MSTRG.10741 & & -2.020 & $2.31 \mathrm{E}-02$ \\
\hline LOC408780 & $\begin{array}{l}\text { signal-induced proliferation-associated 1-like } \\
\text { protein } 2\end{array}$ & -2.040 & $6.97 \mathrm{E}-03$ \\
\hline MSTRG.851 & & -2.069 & $1.42 \mathrm{E}-02$ \\
\hline MSTRG.849 & & -2.093 & $1.32 \mathrm{E}-03$ \\
\hline MSTRG.8420 & & -2.134 & $4.64 \mathrm{E}-02$ \\
\hline LOC100577541 & uncharacterized LOC100577541 & -2.164 & 2.09E-02 \\
\hline MSTRG.14718 & & -2.176 & $4.77 \mathrm{E}-02$ \\
\hline LOC412739 & roundabout homolog 2 & -2.220 & 4.64E-02 \\
\hline MSTRG.13431 & & -2.280 & $4.27 \mathrm{E}-02$ \\
\hline MSTRG.12525 & & -2.281 & $2.87 \mathrm{E}-02$ \\
\hline MSTRG.3576 & & -2.351 & $1.14 \mathrm{E}-02$ \\
\hline LOC107964850 & uncharacterized LOC107964850 & -2.352 & $2.09 \mathrm{E}-02$ \\
\hline LOC726709 & uncharacterized LOC726709 & -2.412 & $3.85 \mathrm{E}-03$ \\
\hline LOC408329 & uncharacterized LOC408329 & -2.671 & $1.33 \mathrm{E}-02$ \\
\hline LOC409608 & peroxidasin & -2.687 & $3.84 \mathrm{E}-03$ \\
\hline MSTRG.848 & & -2.697 & $1.65 \mathrm{E}-03$ \\
\hline LOC107964589 & uncharacterized LOC107964589 & -2.922 & $1.20 \mathrm{E}-02$ \\
\hline MSTRG.13430 & & -3.017 & $4.51 \mathrm{E}-02$ \\
\hline LOC408826 & dystroglycan & -3.186 & $2.69 \mathrm{E}-02$ \\
\hline LOC113219078 & interaptin-like & -3.609 & $4.21 \mathrm{E}-02$ \\
\hline LOC724508 & trichoplein keratin filament-binding protein & -3.694 & $1.18 \mathrm{E}-03$ \\
\hline LOC113219007 & zinc finger protein 524-like & -4.620 & $4.27 \mathrm{E}-02$ \\
\hline LOC107964068 & uncharacterized LOC107964068 & -6.451 & $2.84 \mathrm{E}-02$ \\
\hline LOC100578418 & uncharacterized LOC100578418 & -6.556 & $2.63 \mathrm{E}-03$ \\
\hline LOC113218727 & uncharacterized LOC113218727 & -8.809 & $3.21 \mathrm{E}-06$ \\
\hline LOC113219396 & leucine-rich repeat serine/threonine-protein kinase 1 & -22.693 & $3.14 \mathrm{E}-10$ \\
\hline LOC411557 & terminal nucleotidyltransferase $5 \mathrm{C}$ & -24.771 & $1.82 \mathrm{E}-12$ \\
\hline LOC551123 & RNA-binding protein Musashi homolog Rbp6 & -25.272 & $6.86 \mathrm{E}-13$ \\
\hline
\end{tabular}

\section{Supplemental figures and tables Chapter 5:}


A

During or after treatment

phase $\prec$ after $\diamond$ during
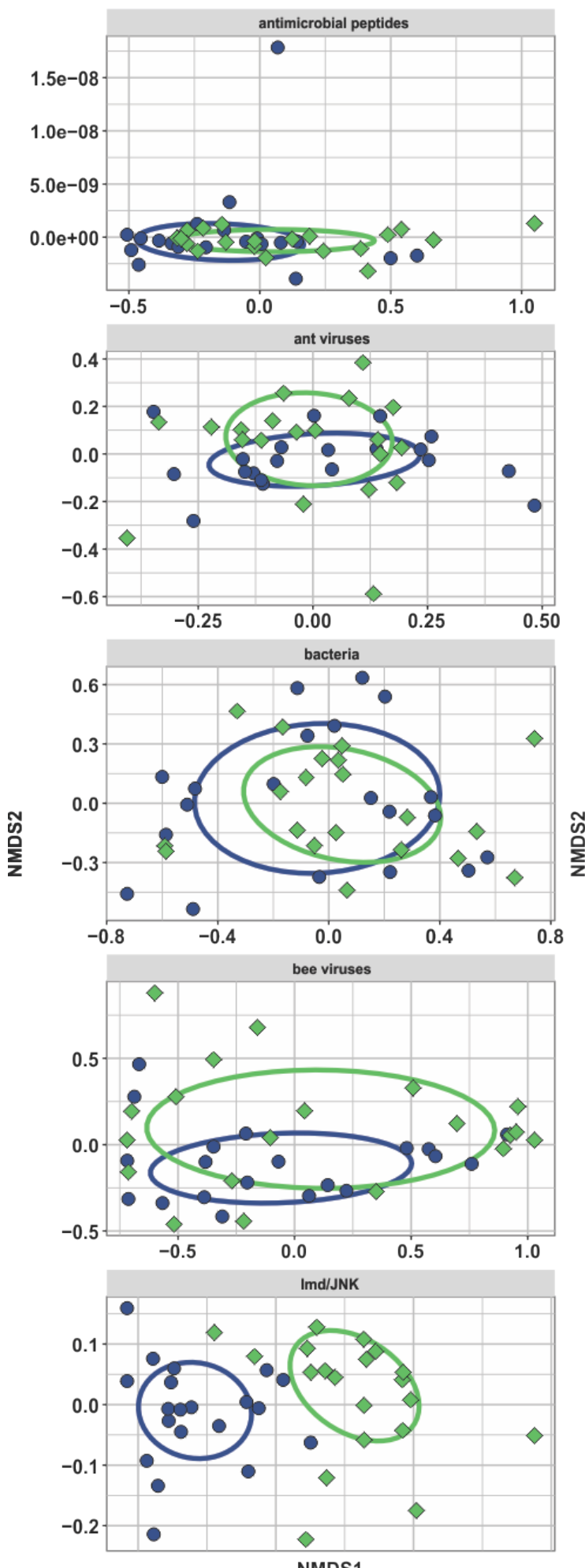

NMDS1
B

Control or dsRNA treatment

group $=$ crtl $\triangle$ dsRNA
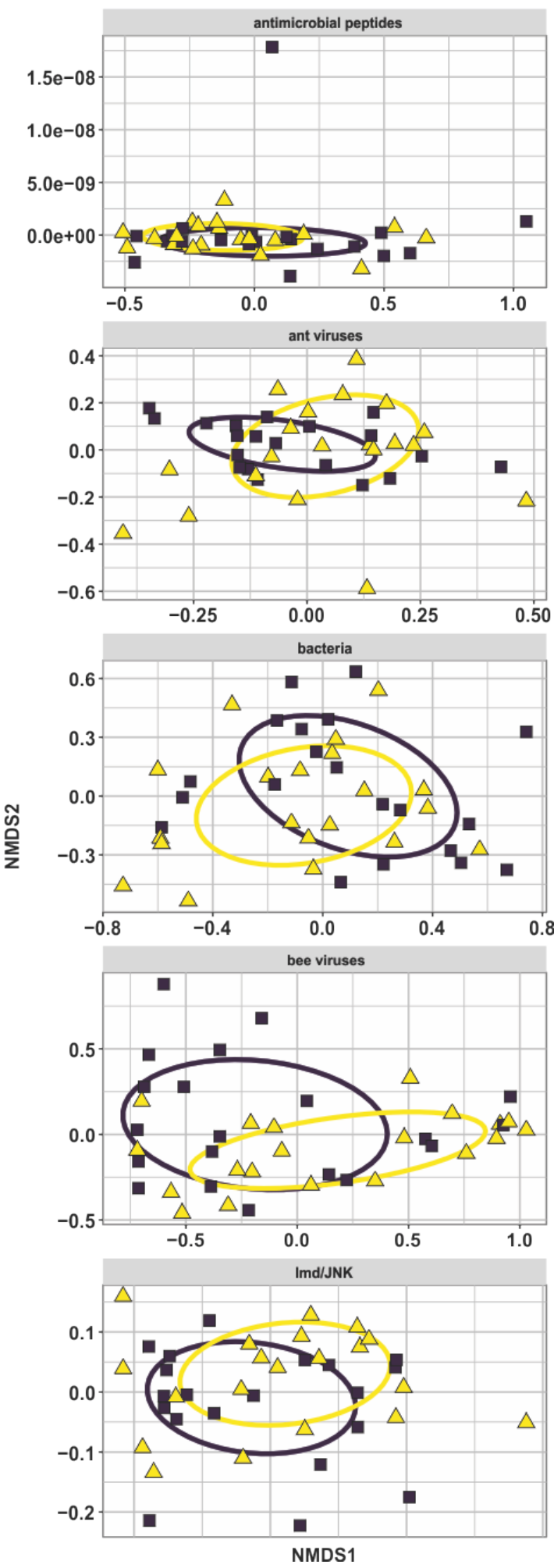

Figure S5.1 continues on next page 

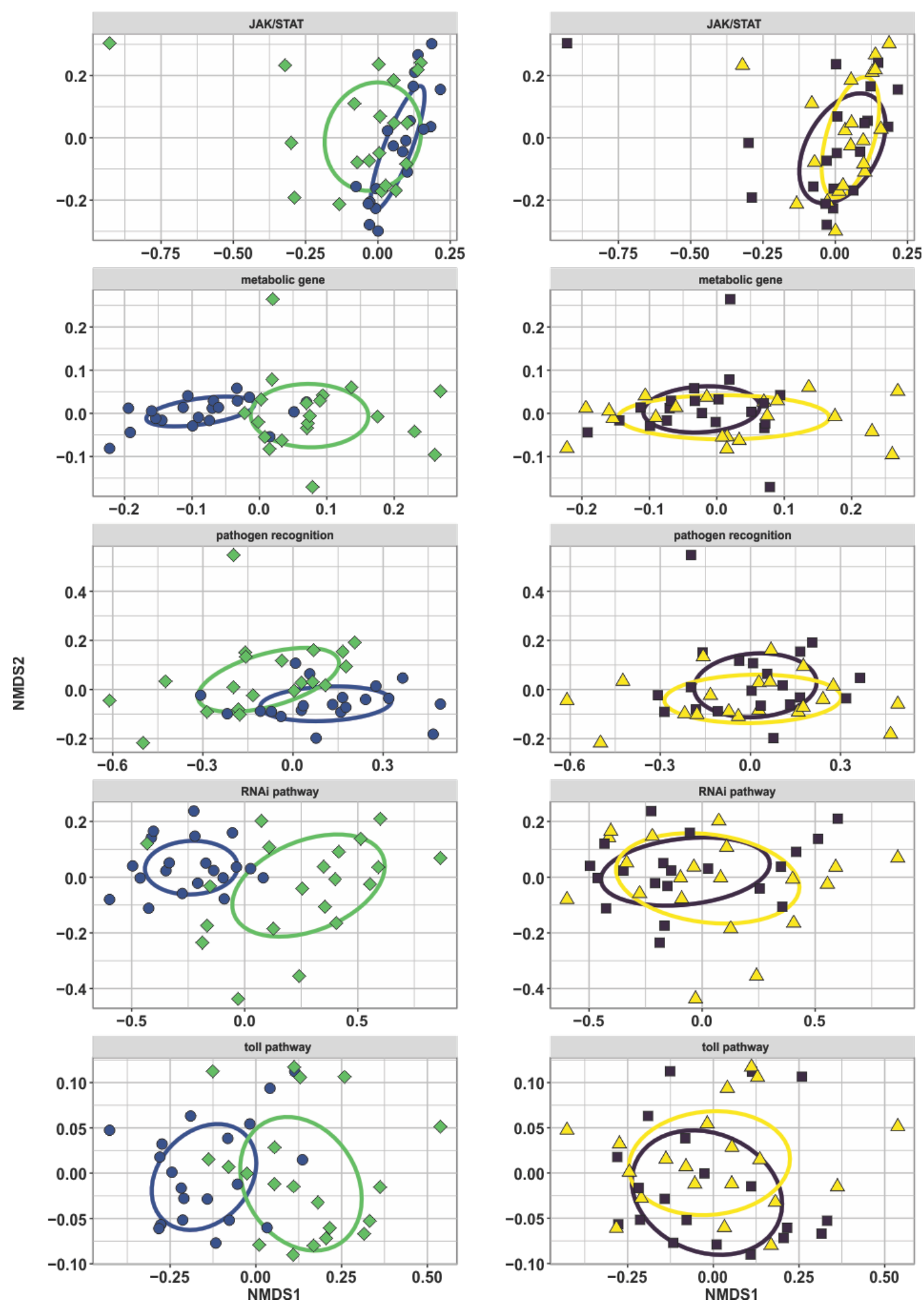

Figure S5.1: Nonmetric multidimensional scaling ordination of ant nest gene expression and pathogen load based on Bray-Curtis dissimilarities on $2^{\Delta \mathrm{Cq}}$ values. Dissimilarities were calculated based on two antimicrobial peptides, two immune deficiency/ c-Jun N-terminal kinases (imd/JNK) genes, three JAK-STAT genes, four pathogen recognition genes, five (RNA interference) RNAi genes, five Toll genes, three bee-associated viruses, nine ant-associated viruses and four bacteria. A: samples during (green diamonds) vs after (blue circles) treatment. B: control (dark purple squares) vs dsRNA treatment (yellow circles). Ellipses indicate 50\% confidence intervals. 

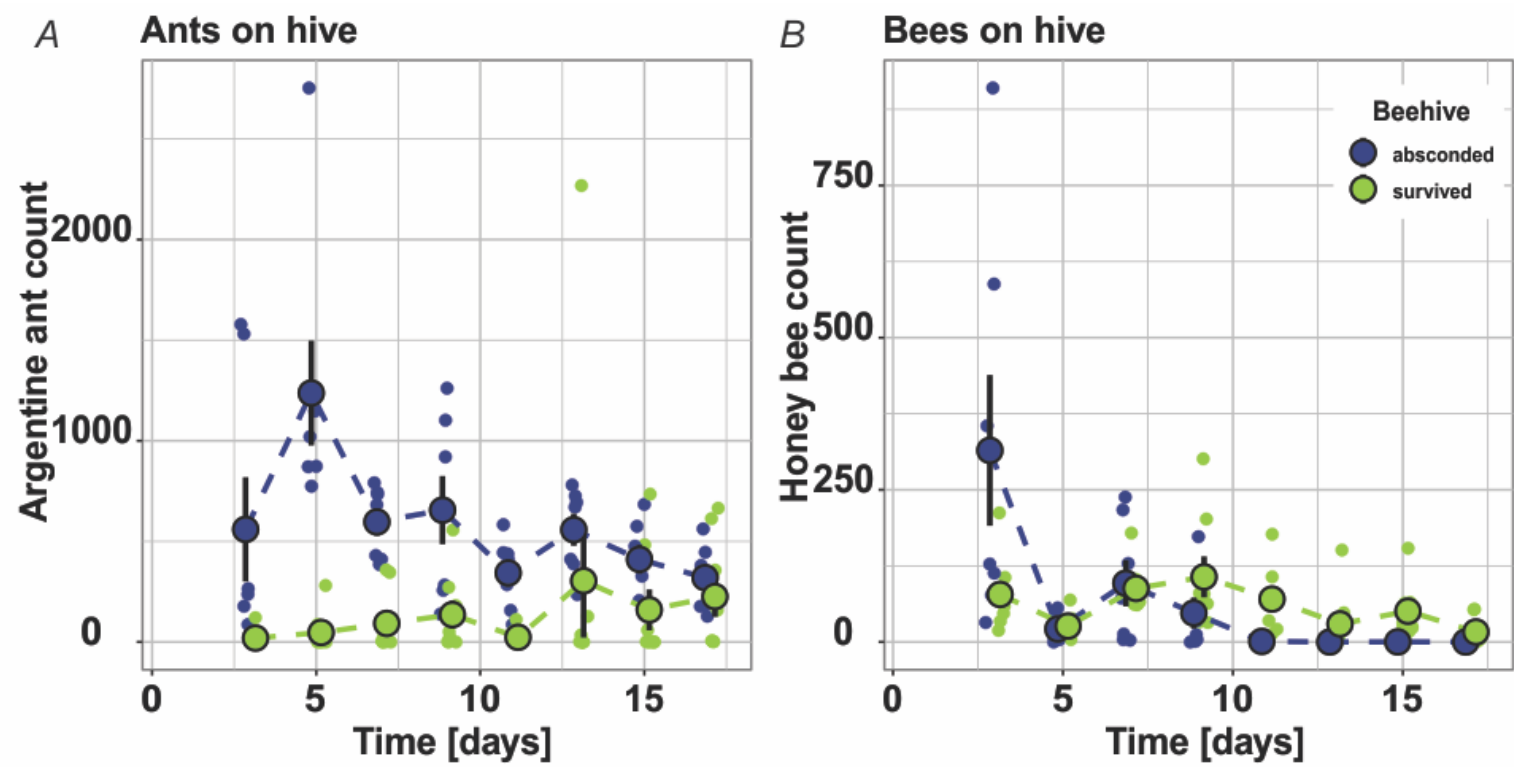

Figure S5.2: The number of Argentine ants (A) and honey bees (B) on the outside of beehives that absconded or survived the course of the experiment. The number of ants and bees were estimated by counting ants and bees on all four sites of the hive box from photos. Hives are grouped into hives that were abandoned by bees during the experiment (absconded, blue) or survived the experiment (green). Argentine ants were treated with sugar water, dsRNA in sugar water or vanquish bait pro which is not shown in this plot, instead, we grouped ants and bees according to the fate of the bee colony.
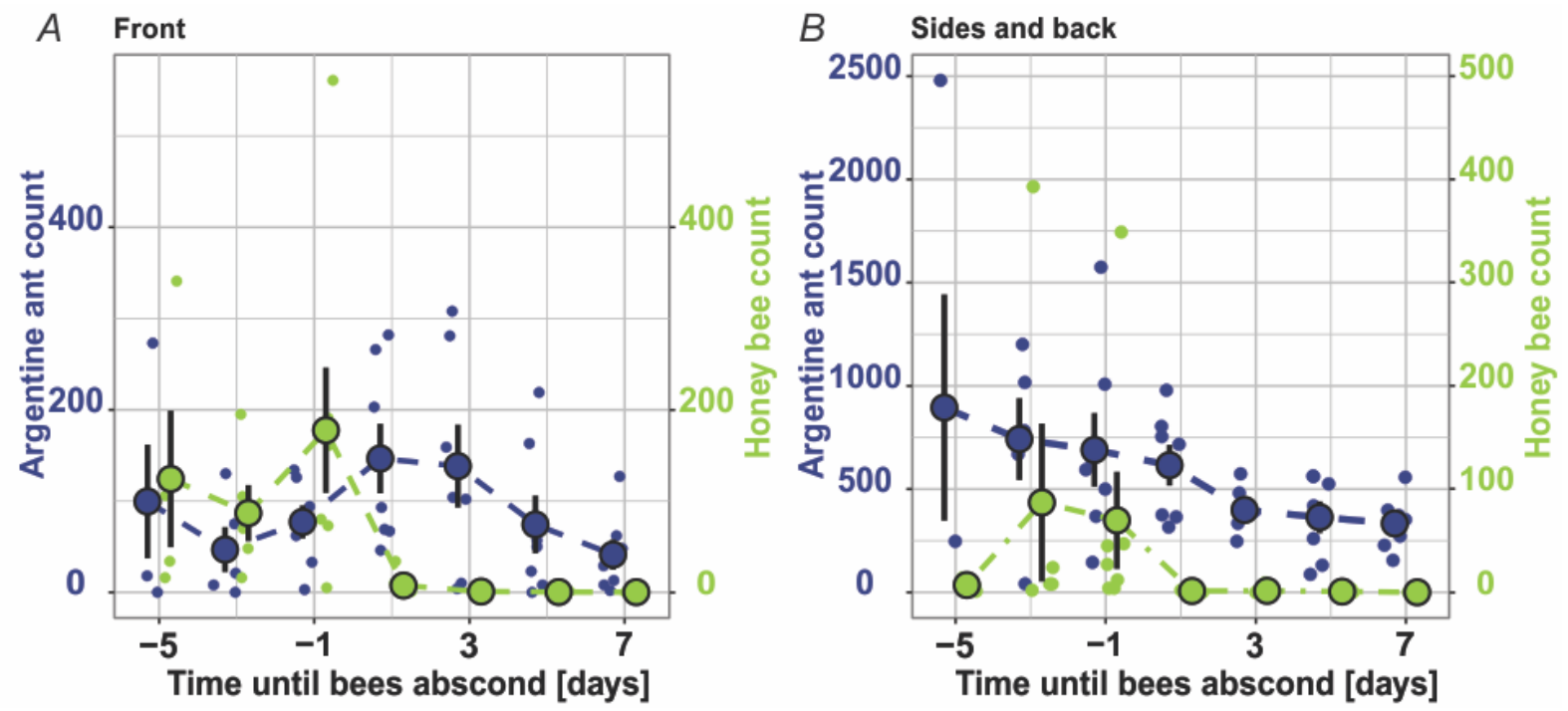

Figure S5.3: Number of Argentine ants and honey bees on beehives that absconded (abandoned their hive) estimated from photo counts. The number of ants on the front of the beehive (A) and the other sides of the beehive (B) are shown in blue (left y-axis) and the number of bees on the front of the beehive (A) and the other sides of the beehive (B) in green (right y-axis) as mean \pm one standard error (large circle with error bar) with individual data points as small circles in the background. Note the different scale of ant and bee counts in plot B. Counts are shown relative to the time the beehive was absconded with negative numbers indicating days before and positive numbers days after bees abandoned their hive. 
Table S5.1: Double-stranded RNA (dsRNA) targets used for Argentine ant control. Sequences were designed to target two genes associated with immunity in Argentine ants, dicer-1 (LOC105668788) and spätzle (LOC105678357).

\begin{tabular}{|c|c|}
\hline $\operatorname{arget}(\mathrm{a}$ & Sequence $\left(5^{\prime}-3^{\prime}\right)$ \\
\hline $\begin{array}{l}\text { Dicer-1 } \\
\text { (XM_012 } \\
361369.1)\end{array}$ & $\begin{array}{l}\text { TTACCCACCTGAAGAATCGGCTATTGGATTTGGCATACTTACGTCGAAGG } \\
\text { AAATACCTAAGTTGTGTCCATTTCCTATTTATACTAGGTCGGGTGAGGTT } \\
\text { CATGTGCAATTAAAACTTAGTAAGCAGACTGTAATTTTGGACGAGGTTCA } \\
\text { GATAGAAAGAGTTGATACTTTTCTTAATTATACCTTCACAAACGTCTTGA } \\
\text { GATTACAAAAGTATCTGATGCTCTTCGATCCAAACACTTCAGAGAATTCA } \\
\text { TACATAATTGTGCCAGTAAAAATATTTACAACAGAAGAAGGGTCAGATGT } \\
\text { TAGTGTGGACTGGGATTTTCTGGAATGCATTTATCAGAATCAAAATGCGG } \\
\text { TGCCAACCAAAGTTCCAGAAGAAGATAGGAAAAATTTCAACTTTGATCCA } \\
\text { TCCAAATATTATGACGCTGTAATCATGCCATGGTACAGGAGTCAAGACCA } \\
\text { ACCACAGTACTTTTATGTAGCGGAAATCTGCACGAACCTAAATCCGAAG }\end{array}$ \\
\hline $\begin{array}{l}\text { Spätzle } \\
\text { (XM_012 } \\
377618.1 \\
\text { and } \\
620.1)\end{array}$ & $\begin{array}{l}\text { CAGATTGCGAACACGAAATGGTGGTAGAAGTTACGTAAAAACTAAAATTG } \\
\text { ACGAAACCGCGCCACGCTGTGACAACGGCCAACTCTTTTGCGAAAATGTT } \\
\text { CAAAATTATCCCAGAGAGTTTGTAAACCAAATACTTAAGAAAAATTCCGA } \\
\text { TTTGTATGTTTACGCGTTTAAGGATGCTGTGCCTATTGACTCAAGATTAG } \\
\text { ATTTAGAAGACGTAAAAGAACTTTGTCCGTCCACGGGACATGATATTATT } \\
\text { CCGGAAGCGGCTAAAAGTATGAATGGTGAATGGATGCTTATCGTGCAATC } \\
\text { GAAAGAGATGAATTTCAACAGCGTATACATATTGAAACATGCCAAGAAC } \\
\text { AAGATAGCAAATGCCAAATAATCAACGACAAGCTACCCGAAGGTTACTTT } \\
\text { CCACGGTGCAAACAGAAATATATTTATCGGCAACTATTAGCTATCAAGGA } \\
\text { CAATGTGAAAACTGTCCACGAAAGCTTTCCGTTTCCTGCGACTTGTTGCT } \\
\end{array}$ \\
\hline
\end{tabular}


Table S5.2: TaqMan array targets used to measure gene expression and pathogen levels in Argentine ants. TaqMan assays were designed using Primer Express software v3.0.1 *except from the BQCV assay, which was originally designed by Chantawannakul et al 2006. TaqMan Array Micro Fluidic Cards contained 47 custom TaqMan assays that included 16 viruses, four bacteria, 22 immune-associated genes, four metabolic genes and three genes used as endogenous controls. Targets are shown with acronyms in square brackets, if applicable. A subset of 27 essays was previously tested on pooled Argentine ant complementary DNA to confirm amplification and are indicated by $\checkmark$. Sequences (F: forward, R: reverse, P: probe) are given in 3' -5 ' direction and amplicon product size is shown in base pairs (bp). Putative genes or viruses are indicated by **. Protein acronyms: Fadd: fas-associated death domain protein, MyD88-like: myeloid differentiation primary response protein-like, PPI: peptidyl-prolyl cistrans isomerase-like.

\begin{tabular}{|c|c|c|c|}
\hline Target & $\begin{array}{l}\text { GenBank } \\
\text { Accession }\end{array}$ & Primer and probe sequence & $\begin{array}{l}\text { Prod } \\
{[\mathrm{bp}]}\end{array}$ \\
\hline \multicolumn{4}{|l|}{ Viruses } \\
\hline \multirow{3}{*}{$\begin{array}{l}\text { Deformed wing } \\
\text { virus [DWV] }(\checkmark)\end{array}$} & \multirow{3}{*}{ NC_004830 } & F AAATACTGTAAAGTGGCGGACGTT & \multirow{3}{*}{118} \\
\hline & & R CGTTCCTTCTACCGAGACCTTGT & \\
\hline & & $\begin{array}{l}\text { P } \\
\text { TAAAACATCCAACTAGACCTGT }\end{array}$ & \\
\hline \multirow{3}{*}{$\begin{array}{l}\text { Kashmir bee virus } \\
{[\mathrm{KBV}](\checkmark)}\end{array}$} & \multirow{3}{*}{$\begin{array}{l}\text { NC } 004807 \\
\text { AY } 275710\end{array}$} & F GCATCGAGCGCATTCCA & \multirow{3}{*}{86} \\
\hline & & R TACACCTCCTACAATATCAGCAAACC & \\
\hline & & P TGCAAAGCCGGTAACT & \\
\hline \multirow{3}{*}{$\begin{array}{l}\text { Linepithema } \\
\text { humile virus } 1 \\
{[\text { LHUV1] }(\checkmark)}\end{array}$} & \multirow{3}{*}{ MF417634 } & F AACAGTATGCAGAGACAAGATTCGA & \multirow{3}{*}{71} \\
\hline & & R ATTCAGCTGGAGTACTTGCATGTTA & \\
\hline & & P CCGGACGCACACAA & \\
\hline \multirow{3}{*}{$\begin{array}{l}\text { Black queen cell } \\
\text { virus [BQCV]* }\end{array}$} & \multirow{3}{*}{ NC_003784 } & F GGTGCGGGAGATGATATGGA & \multirow{3}{*}{71} \\
\hline & & R GCCGTCTGAGATGCATGAATAC & \\
\hline & & $\begin{array}{ll}\mathrm{P} & \text { TTTCCATCTTTATCGGTACGCCGCC }\end{array}$ & \\
\hline \multirow{3}{*}{$\begin{array}{l}\text { Acute bee paralysis } \\
\text { virus [ABPV] }\end{array}$} & \multirow{3}{*}{ NC_002548 } & $\begin{array}{l}\text { F GGTATGCTCAGGAGAGATTCCTAAA } \\
\end{array}$ & \multirow{3}{*}{121} \\
\hline & & $\mathrm{R}$ TCCAACACAACAAGCCTTAATACTG & \\
\hline & & P ACACAACATGGTTACCCATA & \\
\hline \multirow{3}{*}{$\begin{array}{l}\text { Linepithema } \\
\text { humile } \mathrm{C} \text { virus } 1 \\
{[\text { LhuCV1] }(\checkmark)}\end{array}$} & \multirow{3}{*}{ MH213244 } & F GGGAGATGGGCTGGAAGTG & \multirow{3}{*}{58} \\
\hline & & R TTGGCTCTCGACACCATCAG & \\
\hline & & P CAGCTGTCGCCCTT & \\
\hline \multirow{3}{*}{$\begin{array}{l}\text { Linepithema } \\
\text { humile rhabdo-like } \\
\text { virus } 1 \text { [LhuRLV1] } \\
(\checkmark)\end{array}$} & \multirow{3}{*}{ MH213246 } & F TGGAACCCAAGAGCATGGAT & \multirow{3}{*}{138} \\
\hline & & R TCTTTGGTACCCCAATTGGATCT & \\
\hline & & P CCTCCCAGATTGTGC & \\
\hline \multirow{3}{*}{$\begin{array}{l}\text { Linepithema } \\
\text { humile bunya-like } \\
\text { virus } 1 \text { [LhuBLV1] }\end{array}$} & \multirow{3}{*}{ MH213237 } & F AAGGTGAATGTTTGTCTGAACTACCA & \multirow{3}{*}{132} \\
\hline & & R AAGACAAGACAGAAGAAGATGCATTG & \\
\hline & & P ATCTCCAACATTGATATAACGTCTA & \\
\hline \multirow{3}{*}{$\begin{array}{l}\text { Linepithema } \\
\text { humile } \\
\text { entomopoxvirus } 1 \\
\text { [LhuEV1] }\end{array}$} & \multirow{3}{*}{ MH213250 } & F TGCAAGTACCAGAGTAGAGAACGGTAT & \multirow{3}{*}{121} \\
\hline & & R AGATGCTTTAGCCTGTCCAGAGTT & \\
\hline & & P TTCGTCGACAATAAAATCCTGAA & \\
\hline \multirow{3}{*}{$\begin{array}{l}\text { Linepithema } \\
\text { humile } \\
\text { polycipivirus } 1 \\
\text { [LhuPcV1] }\end{array}$} & \multirow{3}{*}{ MH213247 } & $\begin{array}{ll}\text { F } & \text { TTTTTTGGCACATCGAACCA } \\
\end{array}$ & \multirow{3}{*}{71} \\
\hline & & R TCAGAGACAGTTCGCTCAATACG & \\
\hline & & P CCAGCCTTACTAACGAT & \\
\hline
\end{tabular}




\begin{tabular}{|c|c|c|c|}
\hline Linepithema & & F CCACGTCCACCTCGTCAATT & \\
\hline humile partiti-like & & R GTTGAAAGAAAAGCGGGCTTAG & \\
\hline $\begin{array}{l}\text { virus } 1 \text { [LhuPLV1] } \\
(\checkmark)\end{array}$ & MH2I3239- 240 & P ACGCAAAACCTCAGGTG & 62 \\
\hline Linepithema & & F TCTTGAGCTTCCTGTGTTAACCAA & \\
\hline humile qinvirus- & MH213241 & R AATGCCGTTTTTGCACCAA & 80 \\
\hline $\begin{array}{l}\text { like virus } 1 \\
\text { [LhuQLV1] }\end{array}$ & MH213241 & P TATACCCCAACCACCTAGG & 80 \\
\hline Linepithema humile & & F CCATGTTCTCGGTGGAGCAT & \\
\hline polycipivirus 2 & MH213248 & R GACACGGGTGGATATAGGCATT & 67 \\
\hline$[\mathrm{LhuPcV} 2]^{* *}$ & & P CCTCTGCGCAGTTAA & \\
\hline Linepithema humile & & F GGATGGATTCACGTCGTTCAA & \\
\hline picorna-like virus 1 & MH213235 & R AACCGTCCGGCTTGGAA & 78 \\
\hline$\left[\right.$ LhuPiLV1] ${ }^{* *}$ & & P CAACGTGCGTTCTC & \\
\hline Linepithema humile & & F GAGCAGCAATGGCGAAGAC & \\
\hline toti-like virus 1 & MH213243 & R TCCTCAAATGCTGTACCGCTATC & 76 \\
\hline$[$ LhuTLV1] $(\checkmark)$ & & P CCTAGAGGACTATCGCACAA & \\
\hline & & F AAGAGGCGCTGGAAGTCATC & \\
\hline Pseudomonas & $\mathrm{NC} 029066$ & R TGATCCGCCACGATTGC & 55 \\
\hline & & P CCGCAACCTGTCCGA & \\
\hline $\begin{array}{l}\text { Bacteria } \\
\text { (strain) }\end{array}$ & $\begin{array}{l}\text { Accession } \\
\text { (region) }\end{array}$ & & \\
\hline Pseudomonas & & F CACGCCGTAAACGATGTCAA & \\
\hline (based on $\mathrm{Y} 6$, but & MH997630 & R AGCTGCGCCACTAAAATCTCA & 58 \\
\hline not specific to) $(\checkmark)$ & & P TAGCCGTTGGAATCC & \\
\hline & & F CGTTCTCAGTTCGGATTGCA & \\
\hline Lactobacillus & AB498046 & R CGATTACTAGCGATTCCAACTTCA & 62 \\
\hline & & P CTGCAACTCGCCTGC & \\
\hline Serratia & NR_025341 (16S & F CACGGAGTCGAGTTGCAGACT & \\
\hline proteamaculans & rRNA) (based on, & R AGCCAGCGGACCTCATAAAGT & 61 \\
\hline$(\mathrm{DSM} 4543)(\checkmark)$ & not specific to) & P CGATCCGGACTACGACG & \\
\hline & AJ303011 (16S & F CCGTCACACCATGGGAGTTT & \\
\hline Acinetobacter spp. & rRNA) (based on, & R ACCGCCCTCTTTGCAGTTAG & 61 \\
\hline & not specific to) & P TTGCACCAGAAGTAGC & \\
\hline Immune genes & Accession & & \\
\hline & & $\begin{array}{ll}\text { F } & \text { CAGAGCGGCGGTTCTGA } \\
\end{array}$ & \\
\hline protein Toll $(A)(\checkmark)$ & LOC105680007 & R GGTGAATTGATACAGCTGACTTTTATG & 107 \\
\hline & & $\begin{array}{ll}\mathrm{P} & \text { ATTTTGTGTAACGCCTATTT } \\
\end{array}$ & \\
\hline & & F TGTCTCCGATTCCCGTTCTG & \\
\hline protein Toll $(\mathrm{B})(\checkmark)$ & LOC105674053 & R TTGCTCCTTACGTAACGCGATT & 146 \\
\hline & & P AATAACGGCAGCTCTAC & \\
\hline & & F CTACACTATTTTTGCTGCTCTCCATT & \\
\hline protein spätzle $(\checkmark)$ & LOC105678357 & R GGTTGCTCAGCCATTTTTGAG & 136 \\
\hline & & $\begin{array}{ll}\mathrm{P} & \text { TTTTACCGATAATCGAAGCTTA } \\
\end{array}$ & \\
\hline & & F AGCCTATTGTGTCCGATCCTATTT & \\
\hline $\begin{array}{l}\text { embryonic polarity } \\
\text { protein dorsal }\end{array}$ & LOC105677755 & R TGCGCTACAATGGCTCAGTT & 80 \\
\hline & & P ATAAAAAGGCCATGTCAGATC & \\
\hline
\end{tabular}




\begin{tabular}{|c|c|c|c|}
\hline \multirow{3}{*}{$\begin{array}{l}\text { endoribonuclease } \\
\text { dcr-1 }(\checkmark)\end{array}$} & \multirow{3}{*}{ LOC105668788 } & F CTGTTCTTTACGGCTTCCCATAA & \multirow{3}{*}{64} \\
\hline & & R CGGCATTGGGTAAGACGTAAC & \\
\hline & & $\begin{array}{ll}\text { P } & \text { CTCACCACTGAAATAT } \\
\end{array}$ & \\
\hline \multirow{3}{*}{$\begin{array}{l}\text { endoribonuclease } \\
\text { dicer }(\checkmark)\end{array}$} & \multirow{3}{*}{ LOC105670277 } & F CCCCAAATGCGACCTAAGC & \multirow{3}{*}{101} \\
\hline & & R GACTTGCTACCACCATATATTCCTGTTT & \\
\hline & & P CTTCCAAGTTAATTGCTATTCT & \\
\hline \multirow{3}{*}{$\begin{array}{l}\text { protein argonaute-2 } \\
\text { (A) }(\checkmark)\end{array}$} & \multirow{3}{*}{ LOC105677214 } & F AGGGACCGGTGGATTTTCAT & \multirow{3}{*}{66} \\
\hline & & $\mathrm{R}$ TGAGCAACGTCGACATTTAAGTATG & \\
\hline & & P ACCATACCTGGCTGGAA & \\
\hline \multirow{3}{*}{$\begin{array}{l}\text { protein argonaute-2 } \\
\text { (B) }(\checkmark)\end{array}$} & \multirow{3}{*}{ LOC105669925 } & $\begin{array}{ll}\mathrm{F} & \text { TTCAGCAACATAGGCAGGAAATTAT } \\
\end{array}$ & \multirow{3}{*}{69} \\
\hline & & R AAAACATTATCAGCAATTCCCTAACC & \\
\hline & & $\begin{array}{ll}\text { P } & \text { TCGAACTGAGCTCCTG } \\
\end{array}$ & \\
\hline \multirow{3}{*}{ protein argonaute- 3} & \multirow{3}{*}{ LOC105679318 } & F ACTGGTGAGAAGCAGGAAAGAATAA & \multirow{3}{*}{66} \\
\hline & & R CGTAAGGCCAGCGCAGTAG & \\
\hline & & P CAATTCCGGAACCAGAAG & \\
\hline \multirow{3}{*}{ defensin-2 $(\checkmark)$} & \multirow{3}{*}{ LOC105675717 } & F TCACCGTTTATTGATGGAAATTACTT & \multirow{3}{*}{94} \\
\hline & & R GGCTGACGTAAACAAAAGCTGTT & \\
\hline & & P AATCGTTCTCGTCGCGC & \\
\hline \multirow{3}{*}{$\begin{array}{l}\text { caspase-3 [dredd] } \\
(\checkmark)\end{array}$} & \multirow{3}{*}{ LOC105678455 } & F CAGCAGATATACGGCAATTTACAAA & \multirow{3}{*}{91} \\
\hline & & R CATGAGCCTTTTTCTTTATGACGTATT & \\
\hline & & P TTCATATTTATGTCGACAATGC & \\
\hline \multirow{3}{*}{$\begin{array}{l}\text { tyrosine-protein } \\
\text { kinase hopscotch } \\
(\checkmark)\end{array}$} & & F CGGGCGCTCTAGTCAGATTATAC & \\
\hline & LOC105677920 & R ACCAATTCCAGCAGCATTCC & 67 \\
\hline & & P ATAACTGTGGCCCCAACA & \\
\hline signal transducer \& & & F CATCAGGCGACGAGACAACTT & \\
\hline activator of transcr. & LOC105672556 & R CCGCTTAATCTTTTTCAGTTGCA & 63 \\
\hline $5 \mathrm{~B}[\mathrm{Stat} 5 \mathrm{~B}] * * *$ & & P CAGTGAGCTTCCGCAAC & \\
\hline & & $\begin{array}{ll}\mathrm{F} & \text { TGTGCAAATTACGAATAAAGGAA } \\
\end{array}$ & \\
\hline cytokine & LOC105676117 & R TCAGGAGCGGTTCCAATTG & 82 \\
\hline & & P CTCGCAGAACCGAAC & \\
\hline & & F CGTATATGCGAGCGCTGAGA & \\
\hline Uncharacterised/ & LOC105670591 & R GGGTTCTTCGAAACGTATTGATG & 70 \\
\hline & & $\mathrm{P}$ ACCATCAACGACTGGTCAG & \\
\hline & & F ACCTAATTTTCTCAGACATCCAACAA & \\
\hline MyD88-like & LOC105677438 & R ACGTCTTCCTGTTGACACTTTTTATATAA & 123 \\
\hline & & P CTCTAAGTATCGAAAAAC & \\
\hline & & F CTCGATTATGAGAATTGGCTGAAG & \\
\hline Fadd & LOC105676271 & R CGACCCTGAAGCTATTTCATTATGT & 84 \\
\hline & & P CTGTGCCTACATCACA & \\
\hline$\beta$-1,3-glucan- & & F GAAGATAATTTTCGCACTCTCAACAC & \\
\hline binding protein-like & LOC105673881 & $\mathrm{R}$ TCATAATCCGGATCTAATGGCATT & 74 \\
\hline (A) & & P ATCTCACGTTTCCACAATG & \\
\hline$\beta$-1,3-glucan- & & $\begin{array}{ll}\mathrm{F} & \text { TGCGTCACGCGGAAGTT } \\
\end{array}$ & \\
\hline binding protein-like & LOC105674418 & R GGCACTCTTCCGTACCGATCT & 62 \\
\hline & & P AATCTTGGACAATTGCAC & \\
\hline peptidoglycan- & & F CGGCGTGCGCCAATTTT & \\
\hline recognition protein & LOC105678063 & R TCGACGAAACGTACCGAATCTAC & \\
\hline LC-like $(\checkmark)$ & & P TCGCTCGAGGGTGTAATA & \\
\hline peptidoglycan & & F TTTTTTTCGGAATCCATCTTGTGT & \\
\hline recognition protein- & LOC 105672853 & R CCGCAGCATCACTTTTATCATG & 92 \\
\hline & & P CTTGTGACTCCAATAAGT & \\
\hline
\end{tabular}




\begin{tabular}{|c|c|c|c|}
\hline Metabolic genes & Accession & & \\
\hline \multirow{3}{*}{$\begin{array}{l}\text { cytochrome b-c1 } \\
\text { complex subunit } 2 \\
\text { (mitochondrial) }\end{array}$} & \multirow{3}{*}{ LOC105670362 } & F GCGAGCGCCGATCAATT & \multirow{3}{*}{59} \\
\hline & & R AGCCCGGAATCGCTGTAAG & \\
\hline & & P ATCACGGCGTTTAAC & \\
\hline \multirow{3}{*}{$\begin{array}{l}\text { fatty acid synthase- } \\
\text { like }(\checkmark)\end{array}$} & \multirow{3}{*}{ LOC105679506 } & $\begin{array}{ll}\mathrm{F} & \text { TTCAGATAACCGACGGAACCA } \\
\end{array}$ & \multirow{3}{*}{87} \\
\hline & & R ACGGTCCTTTCAGATCGAATGA & \\
\hline & & $\begin{array}{ll}\text { P } & \text { ATGCTCTAAATCTGCAGCGG } \\
\end{array}$ & \\
\hline \multirow{3}{*}{ guanylate kinase } & \multirow{3}{*}{ LOC105674201 } & F CAAACGATGCACCTCTTCGA & \multirow{3}{*}{85} \\
\hline & & R GAACGGCGAGTTTATTGAAAATG & \\
\hline & & $\mathrm{P}$ CCTTTTTGCTTGTCCC & \\
\hline Reference genes & Accession & & \\
\hline \multirow{3}{*}{$\operatorname{PPI}(\checkmark)$} & \multirow{3}{*}{ LOC105675712) } & $\begin{array}{ll}\text { F } & \text { CGTGATGGAGTTGAGGAAGGA } \\
\end{array}$ & \multirow{3}{*}{62} \\
\hline & & R AGCACAGAGCTCGGAAATTCTC & \\
\hline & & P TTGTCCCTAAAACGGC & \\
\hline \multirow{3}{*}{$\begin{array}{l}\text { dynactin subunit } 4 \\
(\checkmark)\end{array}$} & \multirow{3}{*}{ LOC105671957 } & $\begin{array}{ll}\mathrm{F} & \text { AACTGCGGCTCCTTAAAACCTA } \\
\end{array}$ & \multirow{3}{*}{107} \\
\hline & & R GGACAGTAATGAGAGTCAACCTCTTG & \\
\hline & & $\begin{array}{ll}\text { P } & \text { TTGTCGACACTGCTTGAA } \\
\end{array}$ & \\
\hline \multirow{3}{*}{$\begin{array}{ll}\text { pre-mRNA } & 3 \text { '-end } \\
\text { processing } & \text { factor } \\
\text { FIP1 }(\checkmark) & \end{array}$} & \multirow{3}{*}{ LOC105668404 } & F GGAGTCTGAGATACAAGGTGATGCT & \multirow{3}{*}{117} \\
\hline & & R ACTTCCCTTGCCCCATTTTT & \\
\hline & & P ATCATGAGGAATCCAATTC & \\
\hline
\end{tabular}




\section{Appendices}

\section{Appendix 1:}

Genetic Strain Diversity of Multi-Host RNA Viruses that Infect a Wide Range of

Pollinators and Associates is Shaped by Geographic Origins by Jana Dobelmann, Antoine Felden, and Philip J. Lester as published in Viruses (12) pp 358 
Article

\title{
Genetic Strain Diversity of Multi-Host RNA Viruses that Infect a Wide Range of Pollinators and Associates is Shaped by Geographic Origins
}

\author{
Jana Dobelmann * ${ }^{(\mathbb{D}}$, Antoine Felden $(\mathbb{D}$ and Philip J. Lester $(\mathbb{D}$ \\ School of Biological Sciences, Victoria University of Wellington, Wellington 6012, New Zealand; \\ antoine.felden@vuw.ac.nz (A.F.); phil.lester@vuw.ac.nz (P.J.L.) \\ * Correspondence: jana.dobelmann@vuw.ac.nz
}

Received: 15 February 2020; Accepted: 21 March 2020; Published: 24 March 2020

\begin{abstract}
Emerging viruses have caused concerns about pollinator population declines, as multi-host RNA viruses may pose a health threat to pollinators and associated arthropods. In order to understand the ecology and impact these viruses have, we studied their host range and determined to what extent host and spatial variation affect strain diversity. Firstly, we used RT-PCR to screen pollinators and associates, including honey bees (Apis mellifera) and invasive Argentine ants (Linepithema humile), for virus presence and replication. We tested for the black queen cell virus (BQCV), deformed wing virus (DWV), and Kashmir bee virus (KBV) that were initially detected in bees, and the two recently discovered Linepithema humile bunya-like virus 1 (LhuBLV1) and Moku virus (MKV). DWV, $\mathrm{KBV}$, and MKV were detected and replicated in a wide range of hosts and commonly co-infected hymenopterans. Secondly, we placed KBV and DWV in a global phylogeny with sequences from various countries and hosts to determine the association of geographic origin and host with shared ancestry. Both phylogenies showed strong geographic rather than host-specific clustering, suggesting frequent inter-species virus transmission. Transmission routes between hosts are largely unknown. Nonetheless, avoiding the introduction of non-native species and diseased pollinators appears important to limit spill overs and disease emergence.
\end{abstract}

Keywords: honey bee virus; pollinator; bee associate; Apis mellifera; Linepithema humile; DWV; KBV; Moku virus; invasive species

\section{Introduction}

Pollinator communities worldwide are declining at an alarming rate [1-3]. Emerging viral pathogens have been considered to be major contributors to pollinator losses alongside other drivers, such as habitat destruction; increased use of pesticides and fertilizers; climate change; and biological factors, such as invasive species $[1,3,4]$. The disruption of pollination services could cause severe effects on modern agriculture and biodiversity [5,6]. Insect pollination accounts for an economic value of over $€ 150$ billion annually, and without insect pollinators, food security would be at risk [5]. While managed honey bees and bumble bees are increasingly used to supplement crop pollination, these species cannot replace pollination by wild insects [7].

Viral infections have been extensively studied in the European honey bee (Apis mellifera) because a number of RNA viruses that are circulating within bee populations have been associated with reduced health and reports of colony collapses [8,9]. Increasing evidence suggests that many of these pathogens are not specific to honey bees and instead are shared between many pollinator species and associated arthropods, including bumble bees and other wild bees [10-12]; bee predators, such as wasps [10,11]; and scavenging insects, such as ants, cockroaches, and beetles [10,13-15]. Many of these host species 
have been found cohabiting in bee hives $[13,15,16]$ or share floral resources with honey bees [17-20]. Although arthropods harbour an enormous diversity of viruses [21,22], little is known about the host range and dynamics of viruses within pollinator communities.

Between-host virus transmission plays a key role in virus epidemics [23]. Emerging infectious disease are commonly caused by pathogens that infect and interact through multiple host species [24]. Emerging RNA viruses are suspected to spill from honey bees into associates and vice versa $[6,19,20,25]$. Due to high mutation rates and short generation times, this class of viruses is likely to infect and adapt to new host species and spread through large populations $[10,26]$. Thus far, transmission routes between species are poorly understood; flowers have been suggested to represent "disease transmission hubs" [27], allowing for transmission without direct interactions among species. Closely related viral strains in several pollinators [10] and clustering of virus strains within geographic regions $[12,13,20]$ indicate that ongoing inter-species virus transmission occurs. In order to improve pollinator conservation, multi-host viruses need to be studied further and outside the Apis genus [6].

Species introduction can alter virus communities through the introduction of new pathogens and though introduced hosts providing reservoirs for existing pathogens [28]. For instance, the global spread of the parasitic mite and bee virus vector, Varroa destructor, affected virus spread and infection levels in honey bees [29] but also caused pathogen shifts in other pollinators and bee predators, such as wasps $[18,30]$. Invasive ants with supercolonial social structures in which many interconnected nests form a cooperative network are suspected to tolerate high pathogen loads and form disease reservoirs [31]. In New Zealand, the invasive Argentine ant (Linepithema humile) has been hypothesized to form such a reservoir for honey bee viruses [15]. Three bee viruses have been identified in this ant species [15,32] and frequent interactions with honey bees through ants robbing the honey or brood [33] may facilitate virus transmission.

In this study, we tested the hypothesis that a range of pollinators and associated arthropods, including honey bees and Argentine ants, are infected by the same viral pathogens. Furthermore, we hypothesised that frequent inter-species viral transmission results in viral phylogenies that are predominantly associated with geographic origin instead of being associated with host species. Firstly, we screened pollinators and associated arthropods for five RNA viruses and tested for virus replication. Most bee-affecting viruses are positive sense single-stranded RNA (+ssRNA) viruses [34,35], including deformed wing virus (DWV), Kashmir bee virus (KBV), black queen cell virus (BQCV), and Moku virus (MKV). However, we also tested for a negative sense ssRNA (-ssRNA) virus that was recently discovered in Argentine ants, the Linepithema humile bunya-like virus 1 (LhuBLV1). Limited knowledge about the host range of these viruses, particularly hosts of 'bee viruses' outside bees, makes identifying hosts and potential reservoirs an important step in understanding virus emergence. Secondly, we quantified to what degree the host species and geographic origin of DWV and KBV strains correlate with shared ancestry. We used phylogenetic analysis and phylogeny-trait correlation of our sequences supplemented with viral sequences retrieved from GenBank from various hosts all over the world. Phylogenies separated by geographic regions with hosts interspersed within these can provide evidence for inter-specific transmission. Alternatively, clustering within hosts species could indicate a high degree of host specificity.

\section{Materials and Methods}

\subsection{Specimen Collections}

We used honey bees (A. mellifera) and Argentine ants (L. humile) as two focal species for this study and sampled these species more frequently than other arthropod species. Adult workers were directly collected from ant nests $(n=32)$ and beehives $(n=51)$ using mouth aspirators or collections jars, respectively. Samples of pollinators and associated arthropods $(n=66)$ were collected using sweep nets or jars when directly collected from abandoned beehives. Collection sites consisted of a mix of agricultural fields, beach edge vegetation strips, and urban areas in the Northland region of New 
Zealand (Figure S1). A total of 149 samples were collected in April $2018(n=135)$ and January 2019 $(n=14)$, some in apiaries, some near Argentine ant nests, and others without an apiary or ant nest nearby (details in Figure S1). Specimens were immediately snap frozen in the field at approximately $-150{ }^{\circ} \mathrm{C}$ in a liquid nitrogen dry shipper, before being stored at $-80^{\circ} \mathrm{C}$ in the laboratory until processing. Once in the laboratory, samples were removed from the freezer and placed on ice for identification. When possible, samples were identified to the species level and then again moved to $-80{ }^{\circ} \mathrm{C}$ until RNA extraction.

\subsection{RNA Extraction and Reverse Transcription PCR for Virus Detection}

Samples were screened for four + ssRNA viruses, DWV, BQCV, KBV, and MKV, and an -ssRNA virus, LhuBLV1, using RT-PCR. MKV has only recently been discovered in wasps and bees [36], and LhuBLV1 in Argentine ants [37]. A preliminary screening of a pooled sample for the seven Argentine ant viruses discovered by Viljakainen et al. [37] concluded that only the-ssRNA virus LhuBLV1 was hosted by insects other than Argentine ants and was therefore included in the analysis. Individual whole arthropod samples were homogenized in $1000 \mu \mathrm{L}$ (ants in $500 \mu \mathrm{L}$ ) Trizol ${ }^{\mathrm{TM}}$ (Life Technologies, Carlsbad, CA, USA) and RNA was extracted and precipitated using isopropanol following the "Trizol" extraction" protocol as recommended in the COLOSS bee book [38].

RNA was quantified using a NanoDrop ${ }^{\mathrm{TM}}$ spectrophotometer and approximately $500 \mathrm{ng}$ of RNA were treated with perfecta DNase (Quanta BioSciences, Gaithersburg, MD, USA) before reverse transcription to cDNA using qScript (Quanta BioSciences, Gaithersburg, MD, USA) following the manufacturer's instructions. PCR was carried out with MyTaq ${ }^{\mathrm{TM}}$ Mix and MyTaq ${ }^{\mathrm{TM}}$ Red Mix (Bioline, Meridian Bioscience Inc., London, UK). Here, $15-\mu \mathrm{L}$ reaction mixes consisted of $1 \mu \mathrm{L}$ of template (1:20 diluted cDNA) and $0.4 \mu \mathrm{M}$ of each primer. PCR cycling conditions were $1 \mathrm{~min}$ at $95^{\circ} \mathrm{C}$, followed by 35 cycles ( 38 for $\mathrm{KBV}$ ) of $15 \mathrm{~s}$ of $95^{\circ} \mathrm{C}, 15 \mathrm{~s} 55^{\circ} \mathrm{C}, 30 \mathrm{~s} 72{ }^{\circ} \mathrm{C}$, and a final $7 \mathrm{~min}$ of $72{ }^{\circ} \mathrm{C}$. Positive and negative controls were run alongside every PCR. Primer sequences used in this study can be found in the Supplementary Material (Table S1).

RT-PCR products were visualised on a $2 \%$ agarose gel (1.5\% for DWV) stained with SYBR ${ }^{\text {TM }}$ Safe DNA stain (Invitrogen, Life Technologies, Carlsbad, CA, USA) and run alongside a TrackIt 100-bp DNA ladder (Invitrogen, Life Technologies, Carlsbad, CA, USA). A subset of positive samples were cleaned using ExoSAP-IT ${ }^{\mathrm{TM}}$ (Applied Biosystems ${ }^{\mathrm{TM}}$, Waltham, MA, USA) following the manufacturer's instructions and sent for Sanger sequencing to Macrogen Inc (Seoul, South Korea). This subset included at least one virus positive sample for every arthropod species for each virus. Sequences were confirmed using a BLASTn search (www.ncbi.nlm.nih.gov/Blast) against the NCBI nucleotide collection and were submitted to GenBank under the accession numbers MT068447 to MT068476.

\subsection{Negative Strand Detection}

$\mathrm{DWV}, \mathrm{KBV}, \mathrm{MKV}$, and LhuBLV1-positive samples were further analysed for active virus infection. Strand-specific RT-PCR allows for detection of the negative strand, which in +ssRNA viruses (DWV, KBV, MKV) indicates active replication and parasitism of host cells by the virus $[39,40]$. Similarly, the positive strand in an -ssRNA virus (LhuBLV1) functions as mRNA for protein production or as a template to produce new -ssRNA and indicates an active infection [41]. Equal amounts of RNA from virus-positive samples within each species were pooled and tested for negative or positive strand presence.

Reverse transcription (RT) reactions were carried out in the presence of a tagged primer that contains a non-viral sequence (tag) at the $5^{\prime}$ end of the virus-specific primer [39]. The non-viral sequence is then used in the PCR step in combination with a downstream virus-specific primer, so that only cDNA derived from the strand-specific tagged primer is amplified. Super-Script ${ }^{\mathrm{TM}} \mathrm{IV}$ First-Strand Synthesis System (Invitrogen, Life Technologies, Carlsbad, CA, USA) was used to reverse transcribe $500 \mathrm{ng}$ of RNA into cDNA following the recommended COLOSS bee book protocol for strand-specific RT-PCR [42]. To avoid the detection of false positives, the remaining RNA and tagged 
cDNA primers were digested using RNase H (Invitrogen, Life Technologies, Carlsbad, CA, USA) and $10 \mathrm{U}$ Exonuclease-I (Thermo Scientific ${ }^{\mathrm{TM}}$, Waltham, MA, USA) at $37^{\circ} \mathrm{C}$ for $30 \mathrm{~min}$ followed by inactivation at $70^{\circ} \mathrm{C}$ for $15 \mathrm{~min}$ prior to PCR reactions, as described in the COLOSS bee book [42]. PCR was carried out using tag and reverse primer in a $15-\mu \mathrm{L}$ reaction using myTaq ${ }^{\mathrm{TM}}$ Red Mix (Bioline, Meridian Bioscience Inc., London, UK). Cycling was $1 \mathrm{~min}$ at $95^{\circ} \mathrm{C}, 30$ cycles of $15 \mathrm{~s}$ at $95^{\circ} \mathrm{C}, 10 \mathrm{~s}$ at $55{ }^{\circ} \mathrm{C}, 10$ to $30 \mathrm{~s} 72{ }^{\circ} \mathrm{C}$ (depending on product length), and a final $5 \mathrm{~min}$ of $72{ }^{\circ} \mathrm{C}$. The low cycling number was used to reduce the occurrence of unspecific bands. PCR products were visualised on an agarose gel and sequenced to confirm products as described above.

\subsection{Statistical Analyses}

Statistical analyses were performed in R v3.4.1 [43]. Virus prevalence with $95 \%$ confidence intervals in the two focal species was determined using the epiR package [44] with $95 \%$ sensitivity and specificity. Using the prop.test() function within R (R Foundation for Statistical Computing, Vienna, Austria, [43]), we tested if proportions of infected individuals differed between Argentine ants and honey bees.

\subsection{Phylogenetic Analyses}

To conduct an analysis of globally occurring viral variants in different host species, we supplemented DWV and KBV sequences generated in this study with sequences obtained from GenBank (www.ncbi.nlm.nih.gov/genbank/). We used the same genomic regions that were used in the virus detection assay to conduct the phylogenetic analyses, which were a fragment of the RNA-dependent RNA polymerase ( $R d R p$ ) for DWV and the major capsid protein $v p 3$ for KBV. Through a BLASTn search, we retrieved 160 sequences that matched the DWV RdRp fragment. Only DWV-A was included in the analysis because all DWV sequences from our sample in New Zealand matched the DWV-A group. For KBV, 11 sequences matching the vp3 fragment were found and added to the sequences from this study. Most DWV and KBV sequences available on GenBank are from A. mellifera hosts; therefore, we tested an additional 34 Argentine ant samples obtained by Felden et al. (2019) from Argentina, California, and France (details in Table S2) [45] for DWV and KBV. Briefly, RNA was extracted from single ants using the Direct-zol RNA Microprep extraction kit (Zymo Research) following the manufacturer's instructions. Approximately $50 \mathrm{ng}$ of RNA were reverse transcribed using the High-Capacity cDNA Reverse Transcription kit (Applied Biosystems ${ }^{\mathrm{TM}}$ ), tested for DWV and $\mathrm{KBV}$, and sequenced using the methods described above. From these samples, two DWV and nine KBV sequences were obtained and included in the analysis.

Sequences were trimmed and aligned using the ClustalW algorithm [46] in Geneious v11.1.5 [47]. MEGA v.10.1 [48] was used to find the best substitution model using Bayesian information criterion (BIC) scores, determining HKY $+\mathrm{G}$ as the best model for both viruses. Final alignments were $440 \mathrm{bp}$ in the $R d R p$ region for DWV and $360 \mathrm{bp}$ in the $v p 3$ region for KBV.

Dated Bayesian phylogenetic analyses were performed in BEAST 2.2.6 [49]. Divergence times were calculated based on a tip-dated coalescent model. Clade probabilities were obtained from the posterior distribution incorporation sampling year information (retrieved from GenBank or original publications) for terminal nodes. Data were run under an uncorrelated lognormal relaxed clock model with gamma-distributed rate heterogeneity and coalescent tree prior with exponential population growth. Bayesian analyses were replicated three times and combined, each with Markov chain Monte Carlo (MCMC) of 150 million generations. Trees were sampled every 10,000 generations, of which the first 10\% were discarded as burn-in. Using TreeAnnotator v1.7.5, we constructed maximum clade credibility (MCC) trees and used FigTree v1.4.4 to visualise trees, including high posterior probabilities over 0.6. Tip-dated phylogenies are commonly used to reconstruct RNA viral evolution [50,51]; however, there was a chance that the sampling date and sampling region in our dataset would be connected. Therefore, we repeated the analysis without using the tip-date calibration and compared dated and non-dated phylogenies. 


\subsection{Phylogeny-Trait Correlation}

Phylogeny-trait association analysis was used to test to which extent phenotypic traits of a viral strain, such as host species or geographic location, are correlated with shared ancestry [52]. Association sndex (AI), parsimony score (PS) [53], and monophyletic clade (MC) scores of dated and non-dated phylogenies were computed using BaTS (Bayesian tip-association significance testing) beta build 2 [52] by comparing a null model of random trait-tip assignments to the known trait distribution. BaTS accounts for phylogenetic uncertainty by testing many trees from the posterior distribution. Trait-tip association analyses were run with 500 replicates (500 random trees from the dated Bayesian phylogenetic analysis) to estimate a null distribution for each statistic.

\section{Results}

\subsection{Viral Prevalence in Pollinators and Associated Arthropods}

We collected arthropods from eight different orders. Virus-positive species were found in all except the Odonata (Table 1), but we note that only two samples were taken from this order. Overall, viral infections were common in pollinators and associates, with $83 \%$ (95\% CI: 76\%-88\%) of samples testing positive for at least one virus and $44 \%$ (95\% CI: 36\%-53\%) having multiple infections. The majority of samples were hymenopterans $(n=105)$, out of which $97 \%$ (95\% CI: $92 \%-99 \%)$ were infected with at least one and many with multiple $(62 \%, 95 \% \mathrm{CI}: 52 \%-73 \%)$ viruses. One individual had as many as four viruses. Outside the order Hymenoptera $(n=46), 51 \%(95 \%$ CI: 37\%-65\%) of samples were found positive for at least one virus but only $6 \%(95 \%$ CI: $2 \%-17 \%$ ) for multiple (two) viruses.

\subsection{Viral Coinfections in Honey Bees and Argentine Ants}

Out of the five viruses tested, up to four were found to co-infect individual workers of Argentine ants and honey bees (Figure 1). The most prevalent viruses were DWV in honey bees $(100 \%, 95 \%$ CI: $94 \%-100 \%$ ) and LhuBLV1 in Argentine ants (78\%, 95\% CI: 61\%-90\%) (Table 2). LhuBLV1 was only detected in Argentine ants and associated arthropods in two sites that had ant nests, indicating that other species can host this virus, or that its detection on other arthropods could have resulted from contamination. Only Argentine ants tested positive for the mRNA intermediate of LhuBLV1, suggesting that this virus may be L. humile specific. Interestingly, all ants that tested positive for DWV were sampled from a nest in an apiary (Figure S1, site WAI). In a test of proportions, DWV was more prevalent in bees than in ants $\left(\mathrm{X}^{2}=35.98, p<0.001\right)$, KBV was more prevalent in ants than in bees $\left(X^{2}=6.69, p=0.010\right)$, and MKV infections were not significantly different between the two species $\left(\mathrm{X}^{2}=0.15, p=0.689\right)$ (Table 2). MKV was found at a high prevalence in Vespinae species $(\mathrm{n}=17,88 \%$ infected, $95 \%$ CI: $65 \%-98 \%)$; this group also showed a KBV prevalence similar to honey bees $(24 \%$, $95 \%$ CI: $8 \%-49 \%)$.

\subsection{Viral Replication within Host Species}

All viruses, except BQCV which was only detected in honey bees, were tested for virus replication, or active virus infections in the case of LhuBLV1. Active LhuBLV1 infection was only found in Argentine ants while all +ssRNA viruses replicated in multiple species (Table 1). Fourteen different species tested positive for DWV; active viral replication could be confirmed for five of them (Table 1). For KBV, 5 out of the 9 virus positive species showed active replication and MKV replication was confirmed in 5 out of 10 species including the Polistes and Vespula species tested (Table 1). The negative strand RT-PCR assay is highly conservative and can only confirm the presence of the negative strand intermediate, which is indicative of viral replication but does not allow the exclusion of the possibility for replication. 
Table 1. Virus presence in pollinators and associated arthropods tested for deformed wing virus (DWV), Kashmir bee virus (KBV), Moku virus (MKV), black queen cell virus (BQCV), and Linepithema humile bunya-like virus 1 (LhuBLV1) using RT PCR. Virus not found in species: -; virus found in species: +; active viral replication confirmed: +/+. Summary in bottom line.

\begin{tabular}{|c|c|c|c|c|c|c|c|c|c|c|}
\hline Order & Family & Genus & Species & Common Name & $n$ & DWV & KBV & MKV & BQCV & LhuBLV1 \\
\hline \multirow{4}{*}{ Araneae } & Salticidae & Helpis & Helpis minitabunda & jumping spider & 1 & + & - & + & - & + \\
\hline & Lycosidae & $L y \cos a$ & - & wolf spider & 1 & - & - & - & - & - \\
\hline & Theridiidae & Steatoda & Steatoda capensis & black cobweb spider & 3 & - & + & $+/+$ & - & - \\
\hline & Thomisidae & Diaea & & flower spider & 4 & - & - & - & - & - \\
\hline \multirow{2}{*}{ Blattodea } & \multirow{2}{*}{ Blattidae } & Celatoblatta & - & cockroach & 9 & $+/+$ & + & + & - & - \\
\hline & & Maoriblatta & - & cockroach & 7 & + & - & + & - & + \\
\hline Coleoptera & Curculionidae & Scolopterus & Scolopterus penicillatus & black spined weevil & 1 & + & - & - & - & - \\
\hline \multirow{3}{*}{ Diptera } & Sarcophagidae & Jantia & Jantia crassipalpis & flesh fly & 2 & - & - & - & - & - \\
\hline & Stratiomyidae & - & - & solider fly & 2 & - & - & - & - & + \\
\hline & Tipulidae & Leptotarsus & - & crane fly & 2 & + & - & - & - & - \\
\hline Dermaptera & Forficulidae & Forficula & Forficula auricularia & European earwig & 2 & + & + & - & - & - \\
\hline \multirow{9}{*}{ Hymenoptera } & \multirow{2}{*}{ Apidae } & Apis & Apis mellifera & honey bee & 51 & $+/+$ & $+/+$ & + & + & - \\
\hline & & Bombus & - & bumble bee & 2 & - & $+/+$ & - & - & - \\
\hline & Formicidae & Linepithema & Linepithema humile & Argentine ant & 32 & $+/+$ & $+/+$ & $+/+$ & - & $+/+$ \\
\hline & Pompilidae & Sphictostethus & Sphictostethus nitidus & golden hunter wasp & 1 & + & - & - & - & - \\
\hline & \multirow{4}{*}{ Vespidae } & \multirow{2}{*}{ Polistes } & Polistes chinensis & Chinese paper wasp & 11 & + & - & $+/+$ & - & - \\
\hline & & & Polistes humilis & Australian paper wasp & 2 & - & - & + & - & - \\
\hline & & \multirow{2}{*}{ Vespula } & Vespula germanica & German wasp & 1 & $+/+$ & + & $+/+$ & - & - \\
\hline & & & Vespula vulgaris & common wasp & 3 & $+/+$ & $+/+$ & $+/+$ & - & - \\
\hline & Ichneumonidae & Xanthocryptus & $\begin{array}{l}\text { Xanthocryptus } \\
\text { novozealandicus }\end{array}$ & lemon tree borer parasite & 1 & + & - & - & - & - \\
\hline Odonata & Lestidae & Austrolestes & Austrolestes colensonis & blue damselfly & 2 & - & - & - & - & - \\
\hline \multirow[t]{3}{*}{ Orthoptera } & Acrididae & Locusta & Locusta migratoria & migratory locust & 1 & - & - & - & - & - \\
\hline & \multirow{2}{*}{ Gryllidae } & Bobilla & - & small field cricket & 6 & + & $+/+$ & - & - & + \\
\hline & & Teleogryllus & Teleogryllus commodus & black field cricket & 4 & - & - & - & - & - \\
\hline \multicolumn{5}{|c|}{ Total virus-positive species (samples) } & $\begin{array}{c}21 \\
(151)\end{array}$ & $14(83)$ & $9(44)$ & $10(53)$ & $1(18)$ & $5(32)$ \\
\hline
\end{tabular}


A

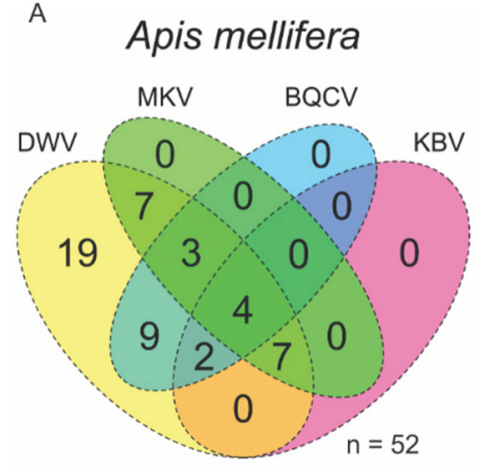

B

Linepithema humile

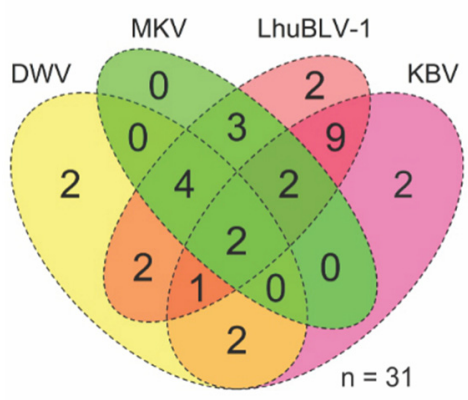

Figure 1. Venn diagram of virus coinfection in honey bees (Apis mellifera) (A) and Argentine ants (Linepithema humile) (B). Most prevalent viruses were DWV in honey bees and LhuBLV1 in Argentine ants. BQCV: black queen cell virus; DWV: deformed wing virus; KBV: Kashmir bee virus; LhuBLV1: Linepithema humile bunya-like virus 1; MKV: Moku virus.

Table 2. Estimated viral prevalence in honey bees (Apis mellifera) and Argentine ants (Linepithema humile) for five virus targets with $95 \%$ confidence intervals (CI) in brackets. - indicates that the virus was not detected in the species and * indicates a significantly higher prevalence in a test of proportions. BQCV: black queen cell virus; DWV: deformed wing virus; KBV: Kashmir bee virus; LhuBLV1: Linepithema humile bunya-like virus 1; MKV: Moku virus.

\begin{tabular}{cccccc}
\hline Host & BQCV & DWV & KBV & LhuBLV1 & MKV \\
\hline A. mellifera & $35 \%(23 \%-49 \%)$ & $100 \% *(94 \%-100 \%)$ & $25 \%(15 \%-39 \%)$ & - & $41 \%(28 \%-55 \%)$ \\
L. humile & - & $41 \%(24 \%-58 \%)$ & $56 \% *(39 \%-72 \%)$ & $78 \%(61 \%-90 \%)$ & $34 \%(20 \%-53 \%)$ \\
\hline
\end{tabular}

\subsection{Viral Strain Diversity and Phylogenetic Analysis}

Using BLAST searches, we confirmed the virus identity and identified locations and hosts infected with viral strains most similar to those observed in our samples. BLAST searches showed that BQCV most closely matched the polyprotein of BQCV found in a honey bee in Lithuania (KP223790) (96\% identity, $100 \%$ query cover). The MKV sequences from 10 different species showed little sequence variation; all sequences derived in this study closely matched (99\%-100\% identity, $97 \%-100 \%$ query cover) the MKV polyprotein found in Vespa velutina in Belgium (MF346349) and Vespula pensylvanica from Hawaii (KU645789). LhuBLV1 most closely matched the putative RdRp complex gene of the only available LhuBLV1 sequences on GenBank (MH213237) from Argentine ants from Spain (100\% identity, $95 \%$ query cover).

The phylogenetic analysis showed that DWV sequences found in New Zealand in this study clustered within the DWV-A clade and formed a monophyletic group with a sequence from $A$. mellifera from New Zealand (MF623172) and Vespa carbo from Italy (KY909333) (posterior probability $(\mathrm{pp})=0.539$, Figure 2). This group is dated to the year 2013 (95\% highest posterior density (hdp) 2010-2016). However, when not including the sampling date in the phylogeny, sequences derived in this study clustered with a broader group of European DWV sequences (Figure S2). Two DWV strains from Pakistan (KP734706, KP734705) represented a relatively old clade $(\mathrm{pp}=1)$ that was estimated to have separated from other DWV sequences around 1977 (95\% hdp = 1965-1989) (Figure 2). The DWV phylogeny further split into two sister groups ( $p p=0.95$, dated to 1986, 95\% hdp $=1979-1994$ ), one from Asia and Europe, which includes the DWV-A variant Kakugo virus, and the other with strains from Europe, North and South America, and New Zealand (Figure 2 and Figure S2, non-dated phylogeny). Within this group, samples from New Zealand all occupy a different clade than samples from the Americas, including Hawaii, and both share these with Europe (pp =1, dated to 1999, 95\% hdp $=1998-2001$, Figure 2 and Figure S2, non-dated phylogeny). Interestingly the two strains collected from Australia (KP734699, KP734632) did not cluster together or with strains from New Zealand. 


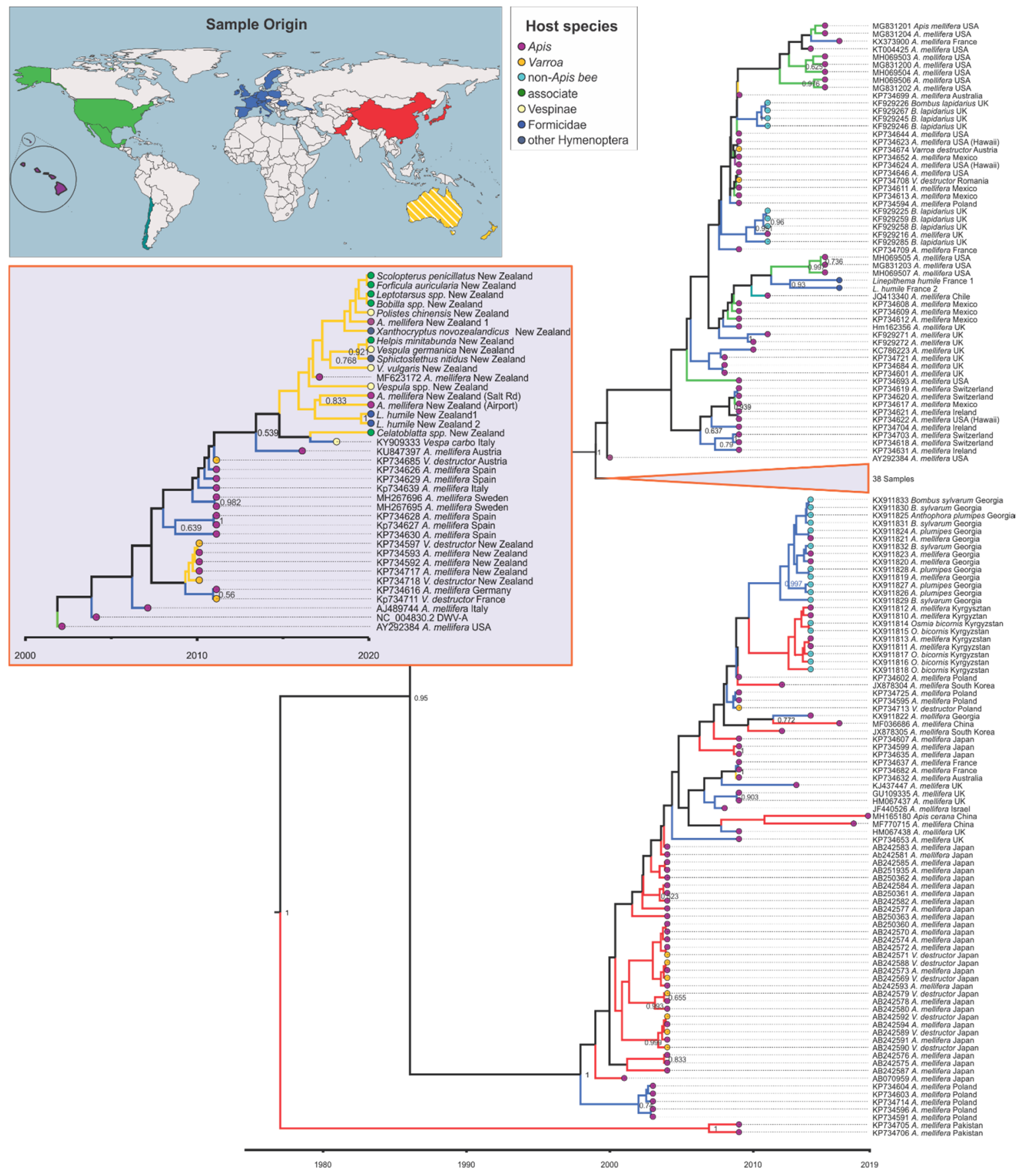

Figure 2. Maximum clade credibility tree for the RNA-dependent RNA polymerase $(R d R p)$ fragment (440 bp) of deformed wing virus (DWV). Grey insert with orange frame shows the collapsed part of the tree that among others includes the 19 samples from New Zealand from this study. Species name, country of origin, and, if applicable, GenBank accession number are given in the branch label. The branches are coloured according to the lineages' inferred geographic origin as shown on the world map and end notes are coloured according to the host group as shown in the host species insert. Posterior support $>0.6$ is given and the $\mathrm{x}$-axis shows time in years.

The phylogenetic analysis for KBV showed that the sister group of most New Zealand KBV sequences was a group from Tasmania ( $\mathrm{pp}=0.641$, Figure 3 and Figure S3, non-dated phylogeny). KBV from a New Zealand V. vulgaris, however, closely matched samples from the USA (HM228885, HM228887) ( $p p=1$, Figure 3 and Figure S3, non-dated phylogeny). 


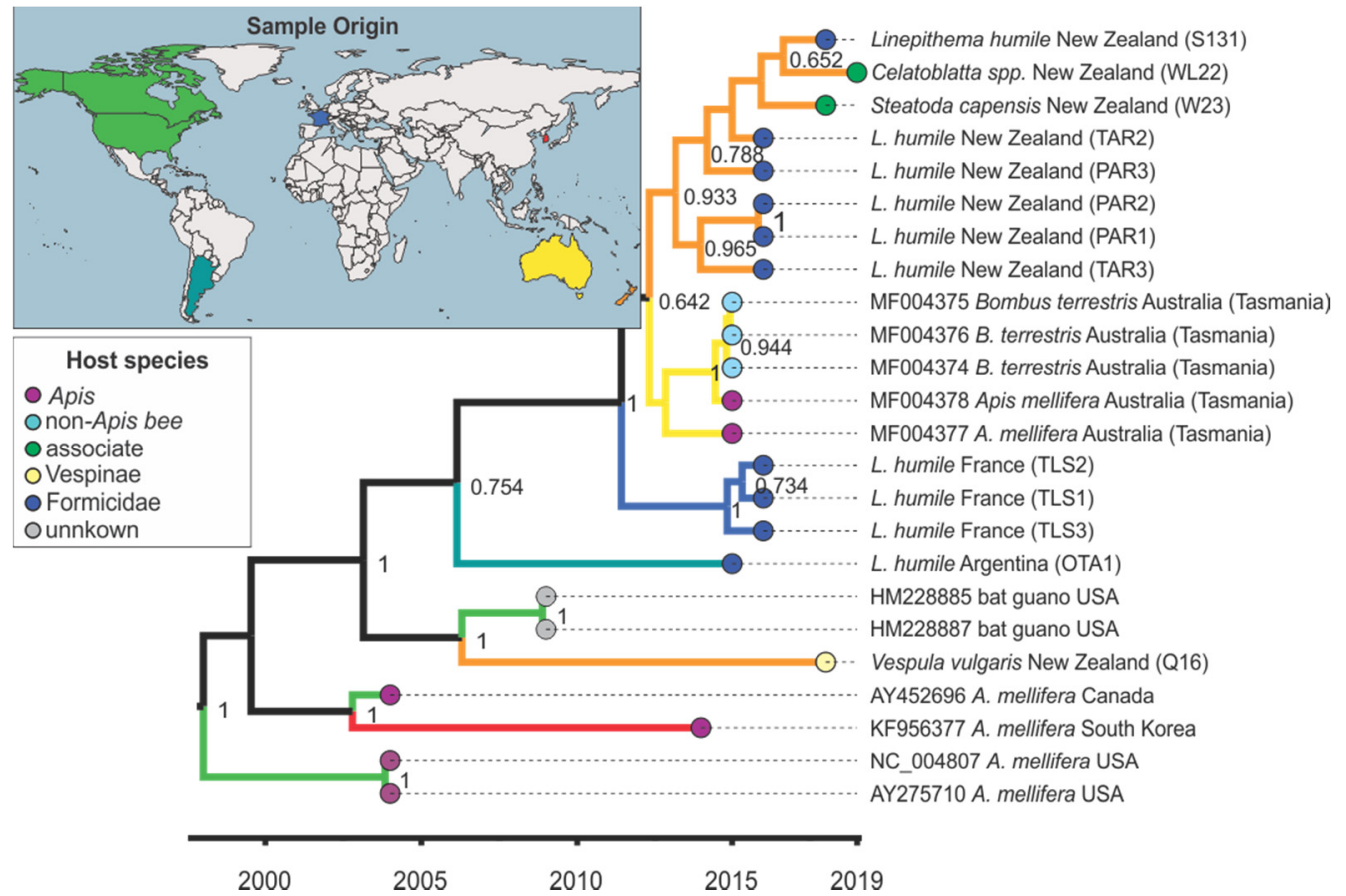

Figure 3. Maximum clade credibility tree for the major capsid protein $v p 3$ fragment $(360 \mathrm{bp})$ of Kashmir bee virus (KBV). Species name, country of origin, and, if applicable, GenBank accession number are given in the branch label. The branches are coloured according to the lineages' inferred geographic origin as shown on the world map and end notes are coloured according to the host group as shown in the host species insert. Posterior support $>0.6$ is given and the $x$-axis shows time in years.

\subsection{Trait-Tip Associations}

The trait-tip association analysis for DWV showed significant clustering within geographic locations and within host species in both dated and non-dated phylogenies (association index (AI) and parsimony score (PS), $p<0.01$, Table 3, and Table S3, trait-tip association in non-dated phylogeny). The PS, which indicates the number of state changes on a tree, was smaller for geographic locations than host species (Table 3 and Table S3). Overall observed to expected ratios indicated a stronger association with geographic origin than with host species (Table 3, Table S3). The maximum monophyletic clade (MC) index tests which traits are associated with phylogeny, and showed that the maximum observed clade size for Asia, Europe, North America, and Oceania was larger than expected by chance (all MC scores $p<0.01$, Table 3 and Table S3). The association was also statistically supported (all MC scores $p<0.05$ ) for a number of host states that included Apis, Varroa, bee associates, non-Apis bees, and $L$. humile (Table 3, bee associates $p=1$ in non-dated analysis, Table S3), which indicates that these groups carry strains that are more closely related than expected due to chance. Yet, it is difficult to determine whether a geographic state or host state causes the association as geographic origin and host species were often linked. For example, all North American samples in the analysis were from A. mellifera hosts and all bee associates were collected in New Zealand. Therefore, host and geographic origin can both contribute to an observed association. 
Table 3. Trait-tip association in deformed wing virus (DWV), as calculated with 500 random trees (replicates) in BaTS (Bayesian tip-association significance testing) [52]. Significant $p$ values are in bold font. Low parsimony score (PS) represents fewer state changes on the tree and stronger trait-tip association. Maximum clade (MC) scores show the maximum size of a clade for a trait state; a high MC is positively correlated with trait-tip association. The geographic region of South America was only represented by one sample and is not shown.

\begin{tabular}{|c|c|c|c|c|c|}
\hline Statistic & n & $\begin{array}{l}\text { Observed to } \\
\text { Expected Ratio } \\
(95 \% \mathrm{CI})\end{array}$ & $\begin{array}{l}\text { Observed Mean } \\
(95 \% \mathrm{CI})\end{array}$ & $\begin{array}{l}\text { Null Mean } \\
\text { (95\% CI) }\end{array}$ & $p$ Value \\
\hline \multicolumn{6}{|c|}{ Association Index $(A I)$} \\
\hline Geographic location & - & $0.21(0.13-0.30)$ & $2.83(1.95-3.74)$ & $13.43(12.47-14.47)$ & $<0.01$ \\
\hline Host species & - & $0.55(0.40-0.74)$ & $4.96(3.89-6.01)$ & $8.98(8.17-9.76)$ & $<0.01$ \\
\hline \multicolumn{6}{|c|}{ Parsimony Score (PS) } \\
\hline Geographic location & - & $0.35(0.30-0.40)$ & $30.91(28.00-34.00)$ & $88.56(84.43-92.45)$ & $<0.01$ \\
\hline Host species & - & $0.68(0.61-0.74)$ & $34.87(32.00-37.00)$ & $51.30(49.75-52.35)$ & $<0.01$ \\
\hline \multicolumn{6}{|c|}{ Maximum Clade (MC) scores } \\
\hline Asia & 50 & - & $15.59(11.00-21.00)$ & $2.57(2.12-3.27)$ & $<0.01$ \\
\hline Europe & 76 & - & $14.44(14.00-16.00)$ & $3.53(2.89-4.79)$ & $<0.01$ \\
\hline North America & 24 & - & $4.48(3.00-8.00)$ & $1.66(1.28-2.09)$ & $<0.01$ \\
\hline Oceania & 25 & - & $12.54(6.00-18.00)$ & $1.72(1.29-2.24)$ & $<0.01$ \\
\hline Hawaii & 3 & - & $1.07(1.00-2.00)$ & $1.01(1.00-1.06)$ & 1 \\
\hline Apis & 126 & - & $13.24(9.00-21.00)$ & 7.04 (5.37-9.93) & 0.01 \\
\hline Varroa destructor & 13 & - & $1.70(1.00-3.00)$ & $1.27(1.01-1.99)$ & 0.04 \\
\hline Associate & 6 & - & $1.83(1.00-3.00)$ & $1.05(1.00-1.19)$ & $<0.01$ \\
\hline Non-Apis bee & 23 & - & $4.23(4.00-5.00)$ & $1.63(1.23-2.07)$ & $<0.01$ \\
\hline Vespidae & 5 & - & $1.09(1.00-2.00)$ & $1.03(1.00-1.15)$ & 1 \\
\hline Formicidae & 4 & - & $2.00(2.00-2.00)$ & $1.02(1.00-1.07)$ & $<0.01$ \\
\hline Other Hymenoptera & 2 & - & $1.00(1.00-1.00)$ & $1.00(1.00-1.00)$ & 1 \\
\hline
\end{tabular}

For $\mathrm{KBV}$, significant clustering within geographic regions and within host species was found (AI and PS, $p<0.01$, Table 4 and Table S4, trait-tip association in non-dated phylogeny). Again, a stronger association with geographic origin was observed than with host species (observed to expected ratio, Table 4). Due to the limited number of available KBV sequences covering the $v p 3$ region $(n=24)$, samples from a geographic location were often sourced from the same host, and vice versa. KBV sequences significantly clustered within New Zealand, Europe, and Australia (all MC: $p<0.05$ ) but also within bumble bees (MC: $p<0.01$ ) (Table 4 and Table S4). The 10 L. humile samples that were positive for KBV did not cluster together (MC: $p=0.16$, Table 4 ) and instead sequences appeared to be divided by geographic region. 
Table 4. Trait-tip associations for Kashmir bee virus (KBV), as calculated with 500 random trees (replicates) in BaTS (Bayesian tip-association significance testing) [52]. Significant $p$ values are in bold font. Low parsimony score represents fewer state changes on the tree and stronger trait-tip association. Maximum clade scores show the maximum size of a clade for a trait state; a high MC is positively correlated with trait-tip association. The geographic regions of Asia of South America were only represented by one sample each and are not shown.

\begin{tabular}{|c|c|c|c|c|c|}
\hline Statistic & $\mathbf{n}$ & $\begin{array}{l}\text { Observed to Expected } \\
\text { Ratio (95\% CI) }\end{array}$ & $\begin{array}{c}\text { Observed } \\
\text { Mean }(95 \% \mathrm{CI})\end{array}$ & $\begin{array}{l}\text { Null Mean } \\
\text { (95\%CI) }\end{array}$ & $p$ Value \\
\hline \multicolumn{6}{|c|}{ Association Index (AI) } \\
\hline Geographic location & & $0.16(0.13-0.25)$ & $0.36(0.33-0.43)$ & $2.19(1.74-2.63)$ & $<0.01$ \\
\hline Host species & & $0.27(0.11-0.50)$ & $0.58(0.29-0.79)$ & $2.14(1.59-2.57)$ & $<0.01$ \\
\hline \multicolumn{6}{|c|}{ Parsimony Score (PS) } \\
\hline Geographic location & & $0.45(0.40-0.58)$ & $6.06(6.00-7.00)$ & $13.44(12.12-14.92)$ & $<0.01$ \\
\hline Host species & & $0.59(0.50-0.73)$ & $7.55(7.00-8.00)$ & $12.74(11.00-13.98)$ & $<0.01$ \\
\hline \multicolumn{6}{|c|}{ Maximum Clade (MC) scores } \\
\hline North America & 4 & - & $2.00(2.00-2.00)$ & $1.26(1.00-2.00)$ & 0.10 \\
\hline New Zealand & 9 & - & $7.83(6.00-7.00)$ & $1.92(1.07-3.00)$ & 0.01 \\
\hline Europe & 3 & - & $3.00(3.00-3.00)$ & $1.10(1.00-2.00)$ & 0.01 \\
\hline Australia & 5 & - & $4.37(4.00-5.00)$ & $1.24(1.00-2.00)$ & 0.01 \\
\hline Apis & 6 & - & $2.54(2.00-4.00)$ & $1.45(1.00-2.94)$ & 0.19 \\
\hline Bombus & 3 & - & $2.97(2.00-3.00)$ & $1.10(1.00-2.00)$ & $<0.01$ \\
\hline Linepithema & 10 & - & $3.08(3.00-4.00)$ & $2.12(1.10-3.44)$ & 0.16 \\
\hline associate & 2 & - & $1.09(1.00-2.00)$ & $1.03(1.00-1.17)$ & 1 \\
\hline
\end{tabular}

\section{Discussion}

Our study shows that multiple RNA viruses that were initially detected in honey bees or Argentine ants infect a range of pollinators and associated arthropods. We have added to the growing body of evidence of bee viruses infecting and replicating in non-Apis hosts. To our knowledge, this work represents the first study to confirm active LhuBLV1 and MKV infections in arthropod hosts by detecting the positive and negative viral strand intermediate, respectively. Virus replication in novel hosts is a key contributor in disease emergence; nevertheless, even hosts without active virus replication may show active virus replication at a different time [54] or can be contaminated with infectious viral particles that contribute to disease spread [6]. The arthropods we examined typically did not display any symptoms of viral infection. The absence of obvious symptoms is common in dicistroviruses like BQCV and KBV [55], yet there may be negative effects on the host. For example, covert KBV infections in bumble bees reduce reproduction [56] and DWV-infected honey bees rarely display wing deformities, although covert infections reduce foraging and long-term survival [20,57]. To which extent these viruses affect hosts other than bees is not well characterised and remains to be investigated [6].

\subsection{Host Range of RNA Viruses}

DWV appears to be the most prevalent honey bee pathogen, occurring in approximately $55 \%$ of colonies worldwide [58]. We found high DWV prevalence in honey bees but also in Argentine ants. The list of DWV hosts is long [58]. We add to it by reporting the first DWV case in the order Orthoptera (Bobilla) and in New Zealand native Hymenoptera (Xanthocryptus novozealandicus and Sphictostethus nitidus), Coleoptera (Scolopterus penicillatus), and Blattodea (Maoriblatta).

In case of the more recently discovered viruses, such as MKV and LhuBLV1, the knowledge of disease symptoms, dynamics, or host range is very limited [36,37]. Studies suggest that the predatory 
wasp V. pensylvanica may represent an MKV reservoir on Hawaii [16,36]. We confirmed active MKV replication in three different wasp species and an overall high prevalence in Vespinae, supporting the potential virus reservoir. Negative sense ssRNA viruses of the Bunyavirales family are found in many species, including LhuBLV1 in Argentine ants [37] and Apis mellifera Bunyavirus 1 and 2 in honey bees [59]. We detected the positive strand intermediate of LhuBLV1 in Argentine ants but not in any other host. LhuBLV1 did not seem specific to Argentine ants, as four other species were found virus positive. Yet, no pollinator species tested positive for this virus, making LhuBLV1 unlikely to be an emerging virus that poses a risk on pollinator communities.

\subsection{Pathogen Reservoir and Viral Spill Over into Wild Populations}

Pathogen transmission may potentially alter competition among multiple host species so that spatial interactions are important when regarding transmission networks [60]. RNA viruses initially identified in honey bees can be widespread in the environment [27], but only some novel hosts acquire infections. High densities of apiaries or introduced ants, such as the Argentine ant, could allow accumulation of high viral levels and possible spill over into pollinators and associates that would account for the virus-positive species found in this study. Frequently, the main drivers for disease emergence in wildlife populations are spill overs from domesticated hosts and human-mediated pathogen invasion, also termed 'pathogen pollution' [61]. Introduced pathogens can provide a competitive benefit to introduced hosts if native hosts are more susceptible to the pathogen than the invader [28]. Moreover, domestic animals often outnumber wild species and can act as reservoir hosts that may drive population decline of the wild host [61]. For instance, the introduction of rabies virus into the Serengeti ecosystem through domestic dogs caused spill overs into wild carnivore communities and ongoing efforts to vaccinate dogs are required to stop the disease spread [62]. In case of the European honey bee, Varroa-mediated high DWV titres in bees may cause spill overs and promote virus spread to associates and other pollinators [63]. The presence of apiaries has shown to cause a high prevalence of DWV and BQCV in wild pollinators [19] and species associated with beehives $[10,13,64]$. We found BQCV in honey bees but no other pollinator, which may be due to the higher BQCV prevalence in honey bees compared to other bees [65], and the low number of wild bee pollinators in our sample set. We only detected DWV in Argentine ants from apiaries, which could indicate that ants acquire the virus from bees, potentially by scavenging in apiaries. In addition to species introduction, anthropogenic actions reducing biodiversity in landscapes and species may facilitate disease transmission and outbreaks [66].

\subsection{Coinfections and Interactions among Viruses}

Coinfections and interactions between viral species within the host can play a key factor in the epidemiology and evolution of viruses [67]. Many insects that we analysed tested positive for multiple viruses. Interactions within hosts and, for example, the order in which pathogens are acquired may have a large positive or negative effect on a secondary infection $[68,69]$. In some cases, infection with one virus can prevent infection with another: Consequently, this makes the infection beneficial if it is less damaging or virulent than the other $[37,70]$. Multiple parasites may compete for resources or even release toxins that inhibit the growth of a competitor [71,72]. These interactions affect adaptive responses to the infection and dynamics within the community [71,73]. Thereby interactions can be strain specific and affect parasite evolution and genetic diversity within virus populations [74]. Future work is needed to understand co-infections and interactions among viruses in addition to the commonly studied host-parasite interactions [68].

\subsection{Multi-Host Viruses in Emerging Disease}

Single-stranded RNA viruses have been considered to be the most likely type of pathogen to jump between species and cause disease outbreaks [23] that could devastate wild pollinator populations. High mutation rates in RNA viruses result in virus populations with high variation amongst genotypes [26]. 
Over time, multi-host parasites can evolve into different species-specialist strains or populations, or remain a generalist parasite with lower than optimal virulence [75]. For multi-host viruses, a lack of host specificity may result in a trade-off between virulence in different hosts that depends on the host quality and availability [75]. Whether a virus that invades a new host causes an emerging disease or only a minor outbreak depends factors, such as its ability to replicate in a novel host and transmission routes $[6,23]$.

We confirmed DWV, KBV, and MKV replication in a number of species, indicating that these species possibly act as biological vectors that facilitate disease emergence. However, with pollinators and associates collected in the field, we cannot exclude the possibility of detecting viruses and negative strand intermediates in the gut contents that come from other infected insects. KBV and MKV replication was confirmed in species not typically known to eat bees, such as Polistes wasps and bumble bees. Some cockroaches, crickets, wasps, and ants that tested positive for virus replication were found in close proximity to beehives and are species known to scavenge or prey on honey bees [30,33]. Recently consumed viral particles from infected honey bees can cause false positives in replication assays. For instance, Varroa mites that exclusively feed on honey bee tissue [76] and vector DWV are assumed to act as a biological vector that propagates DWV [39,77]. Yet, new research suggests that DWV may not replicate in mite cells but in honey bee cells recently consumed by mites [78]. Nevertheless, feeding experiments have shown that scavenger species like the ant Myrmica rubra are found positive for the negative strand of DWV for up to 13 weeks after consuming infected honey bees [14], which indicates that DWV actively infects ants. Whether honey bee scavengers are able to spread viruses without becoming infected themselves [79] or aid in reducing virus transmission by removing infectious carcasses [80] remains to be tested. The detection of negative strand intermediates is an essential step in identifying host species, but only controlled infection experiments allow determination of the virus dynamics in these hosts and the potential for disease emergence.

\subsection{Global Distribution and Evolution of Bee Viruses}

To understand the current epidemic of bee viruses, it is key to determine the role of the host species and geographic distribution $[12,65]$. By placing the sequences generated in this study within a global phylogeny, we found that both KBV and DWV showed strong grouping by geographic regions. Closely related viral strains in geographic locations support the hypothesis that viral infections can be acquired from the environment and other host species in the same location [12]. Although DWV was present in New Zealand before the introduction of Varroa in 2001 [81], all New Zealand DWV strains grouped within the clade that emerged in the early 2000s. The DWV phylogeny showed some broad separations into geographic regions, and only European samples were scattered across groups. Whether European strains are found across the phylogeny because introduced honey bees or other virus-carrying arthropods from Europe initiated the spread remains to be tested further. Research indicates that the global DWV epidemic has been mediated by European A. mellifera populations but that the virus shows little host specificity [63]. Although the KBV phylogeny only represents a limited number of the true KBV diversity, sequences from Argentine ants showed weak association with the phylogeny, suggesting that ants acquired local KBV strains. Moreover, the high prevalence of KBV in this species could indicate a disease reservoir, and ants facilitating further spread of KBV.

\section{Conclusions}

Many emerging viruses infect and actively replicate within a wide range of pollinators and associated Arthropod species. Our results and others indicate that the host species can affect the association with shared ancestry, which indicates virus transmission, but that these effects are weaker than the geographic location [13]. Overall, geographic locations played a major role in shaping patterns of viral genetic diversity. As viruses may be frequently transmitted between species, it is important to incorporate the host abundance and diversity, spatial structure of communities, and multi-host systems into studies of pathogen dynamics and epidemics [60]. Avoiding the introduction of non-native 
species and transport of diseased pollinators is important to stop disease spill overs and prevent disease emergence.

Supplementary Materials: The following are available online at http://www.mdpi.com/1999-4915/12/3/358/s1, Figure S1: Sampling sites in the far north region near Ahipara, New Zealand, Figure S2: Non-dated phylogeny of Deformed wing virus, Figure S3: Non-dated phylogeny of Kashmir bee virus, Table S1: Primers used for virus detection, Table S2: Sampling location of additional Argentine ant samples used for phylogenetic analyses, Table S3: BaTS analysis of non-dated Deformed wing virus phylogeny, Table S4: BaTS analysis of non-dated Kashmir bee virus phylogeny.

Author Contributions: Conceptualization, J.D., A.F. and P.J.L.; methodology, J.D.; formal analysis, J.D.; investigation, J.D.; resources, P.J.L.; data curation, J.D.; visualization, J.D.; writing-original draft preparation, J.D.; writing-review and editing, J.D., A.F. and P.J.L.; funding acquisition, P.J.L. and A.F. All authors have read and agreed to the published version of the manuscript.

Funding: This research was funded by a Royal Society Te Apārangi Marsden Grant 17-VUW-05 to P.J.L. and A.F. and a Victoria University Doctoral Scholarship to J.D.

Acknowledgments: We thank Jess Russell for assistance with sample collections and beekeepers in Northland who provided access to their apiaries. We also thank the three anonymous reviewers whose comments helped improve this manuscript.

Conflicts of Interest: The authors declare no conflict of interest.

\section{References}

1. Potts, S.G.; Biesmeijer, J.C.; Kremen, C.; Neumann, P.; Schweiger, O.; Kunin, W.E. Global pollinator declines: Trends, impacts and drivers. Trends Ecol. Evol. 2010, 25, 345-353. [CrossRef]

2. Hallmann, C.A.; Sorg, M.; Jongejans, E.; Siepel, H.; Hofland, N.; Schwan, H.; Stenmans, W.; Muller, A.; Sumser, H.; Horren, T.; et al. More than 75 percent decline over 27 years in total flying insect biomass in protected areas. PLoS ONE 2017, 12, e0185809. [CrossRef]

3. Sánchez-Bayo, F.; Wyckhuys, K.A.G. Worldwide decline of the entomofauna: A review of its drivers. Biol. Conserv. 2019, 232, 8-27. [CrossRef]

4. Goulson, D.; Nicholls, E.; Botías, C.; Rotheray, E.L. Bee declines driven by combined stress from parasites, pesticides, and lack of flowers. Science 2015, 347, 1255957. [CrossRef]

5. Gallai, N.; Salles, J.M.; Settele, J.; Vaissiere, B.E. Economic valuation of the vulnerability of world agriculture confronted with pollinator decline. Ecol. Econ. 2009, 68, 810-821. [CrossRef]

6. Manley, R.; Boots, M.; Wilfert, L. Emerging viral disease risk to pollinating insects: Ecological, evolutionary and anthropogenic factors. J Appl Ecol 2015, 52, 331-340. [CrossRef]

7. Garibaldi, L.A.; Steffan-Dewenter, I.; Winfree, R.; Aizen, M.A.; Bommarco, R.; Cunningham, S.A.; Kremen, C.; Carvalheiro, L.G.; Harder, L.D.; Afik, O.; et al. Wild pollinators enhance fruit set of crops regardless of honey bee abundance. Science 2013, 339, 1608-1611. [CrossRef]

8. Evans, J.D.; Schwarz, R.S. Bees brought to their knees: Microbes affecting honey bee health. Trends Microbiol. 2011, 19, 614-620. [CrossRef]

9. McMenamin, A.J.; Genersch, E. Honey bee colony losses and associated viruses. Curr. Opin. Insect Sci. 2015, 8, 121-129. [CrossRef]

10. Singh, R.; Levitt, A.L.; Rajotte, E.G.; Holmes, E.C.; Ostiguy, N.; Vanengelsdorp, D.; Lipkin, W.I.; Depamphilis, C.W.; Toth, A.L.; Cox-Foster, D.L. Rna viruses in hymenopteran pollinators: Evidence of inter-taxa virus transmission via pollen and potential impact on non-apis hymenopteran species. PLoS ONE 2010, 5, e14357. [CrossRef]

11. Evison, S.E.; Roberts, K.E.; Laurenson, L.; Pietravalle, S.; Hui, J.; Biesmeijer, J.C.; Smith, J.E.; Budge, G.; Hughes, W.O. Pervasiveness of parasites in pollinators. PLoS ONE 2012, 7, e30641. [CrossRef]

12. Murray, E.A.; Burand, J.; Trikoz, N.; Schnabel, J.; Grab, H.; Danforth, B.N. Viral transmission in honey bees and native bees, supported by a global black queen cell virus phylogeny. Environ. Microbiol. 2019, 21, 972-983. [CrossRef]

13. Levitt, A.L.; Singh, R.; Cox-Foster, D.L.; Rajotte, E.; Hoover, K.; Ostiguy, N.; Holmes, E.C. Cross-species transmission of honey bee viruses in associated arthropods. Virus Res. 2013, 176, 232-240. [CrossRef]

14. Schläppi, D.; Lattrell, P.; Yañez, O.; Chejanovsky, N.; Neumann, P. Foodborne transmission of deformed wing virus to ants (myrmica rubra). Insects 2019, 10, 394. [CrossRef] 
15. Sebastien, A.; Lester, P.J.; Hall, R.J.; Wang, J.; Moore, N.E.; Gruber, M.A. Invasive ants carry novel viruses in their new range and form reservoirs for a honeybee pathogen. Biol. Lett. 2015, 11, 20150610. [CrossRef]

16. Brettell, L.E.; Schroeder, D.C.; Martin, S.J. Rnaseq analysis reveals virus diversity within hawaiian apiary insect communities. Viruses 2019, 11,397. [CrossRef]

17. Bailes, E.J.; Deutsch, K.R.; Bagi, J.; Rondissone, L.; Brown, M.J.F.; Lewis, O.T. First detection of bee viruses in hoverfly (syrphid) pollinators. Biol. Lett. 2018, 14. [CrossRef]

18. Santamaria, J.; Villalobos, E.M.; Brettell, L.E.; Nikaido, S.; Graham, J.R.; Martin, S. Evidence of varroa-mediated deformed wing virus spillover in hawaii. J. Invertebr. Pathol. 2018, 151, 126-130. [CrossRef]

19. Alger, S.A.; Burnham, P.A.; Boncristiani, H.F.; Brody, A.K. Rna virus spillover from managed honeybees (apis mellifera) to wild bumblebees (bombus spp.). PLoS ONE 2019, 14, e0217822. [CrossRef]

20. Fürst, M.A.; McMahon, D.P.; Osborne, J.L.; Paxton, R.J.; Brown, M.J. Disease associations between honeybees and bumblebees as a threat to wild pollinators. Nature 2014, 506, 364-366. [CrossRef]

21. Li, C.X.; Shi, M.; Tian, J.H.; Lin, X.D.; Kang, Y.J.; Chen, L.J.; Qin, X.C.; Xu, J.; Holmes, E.C.; Zhang, Y.Z. Unprecedented genomic diversity of rna viruses in arthropods reveals the ancestry of negative-sense rna viruses. eLife 2015, 4. [CrossRef]

22. Shi, M.; Lin, X.D.; Tian, J.H.; Chen, L.J.; Chen, X.; Li, C.X.; Qin, X.C.; Li, J.; Cao, J.P.; Eden, J.S.; et al. Redefining the invertebrate rna virosphere. Nature 2016, 540, 539. [CrossRef]

23. Woolhouse, M.E.J.; Haydon, D.T.; Antia, R. Emerging pathogens: The epidemiology and evolution of species jumps. Trends Ecol. Evol. 2005, 20, 238-244. [CrossRef]

24. Johnson, P.T.J.; de Roode, J.C.; Fenton, A. Why infectious disease research needs community ecology. Science 2015, 349, 1259504. [CrossRef]

25. Tehel, A.; Brown, M.J.; Paxton, R.J. Impact of managed honey bee viruses on wild bees. Curr. Opin. Virol. 2016, 19, 16-22. [CrossRef]

26. Holmes, E.C. The evolutionary genetics of emerging viruses. Annu. Rev. Ecol. Evol. Syst. 2009, 40, 353-372. [CrossRef]

27. Figueroa, L.L.; Blinder, M.; Grincavitch, C.; Jelinek, A.; Mann, E.K.; Merva, L.A.; Metz, L.E.; Zhao, A.Y.; Irwin, R.E.; McArt, S.H.; et al. Bee pathogen transmission dynamics: Deposition, persistence and acquisition on flowers. Proc. Biol. Sci. 2019, 286, 20190603. [CrossRef]

28. Faillace, C.A.; Lorusso, N.S.; Duffy, S. Overlooking the smallest matter: Viruses impact biological invasions. Ecol. Lett. 2017, 20, 524-538. [CrossRef]

29. Martin, S.J.; Highfield, A.C.; Brettell, L.; Villalobos, E.M.; Budge, G.E.; Powell, M.; Nikaido, S.; Schroeder, D.C. Global honey bee viral landscape altered by a parasitic mite. Science 2012, 336, 1304-1306. [CrossRef]

30. Loope, K.J.; Baty, J.W.; Lester, P.J.; Wilson Rankin, E.E. Pathogen shifts in a honeybee predator following the arrival of the varroa mite. Proc. Biol. Sci. 2019, 286, 20182499. [CrossRef]

31. Cremer, S. Pathogens and disease defense of invasive ants. Curr. Opin. Insect Sci. 2019, 33, 63-68. [CrossRef]

32. Gruber, M.A.M.; Cooling, M.; Baty, J.W.; Buckley, K.; Friedlander, A.; Quinn, O.; Russell, J.; Sebastien, A.; Lester, P.J. Single-stranded rna viruses infecting the invasive argentine ant, linepithema humile. Sci. Rep. 2017, 7, 3304. [CrossRef]

33. Buys, B. Relationships between argentine ants and honeybees in south africa. Relatsh Argent. Ants Honeybees S. Afr. 1990, 519-524.

34. Galbraith, D.A.; Fuller, Z.L.; Ray, A.M.; Brockmann, A.; Frazier, M.; Gikungu, M.W.; Martinez, J.F.I.; Kapheim, K.M.; Kerby, J.T.; Kocher, S.D.; et al. Investigating the viral ecology of global bee communities with high-throughput metagenomics. Sci. Rep. 2018, 8, 8879. [CrossRef]

35. Grozinger, C.M.; Flenniken, M.L. Bee viruses: Ecology, pathogenicity, and impacts. Annu. Rev. Entomol. 2019, 64, 205-226. [CrossRef]

36. Mordecai, G.J.; Brettell, L.E.; Pachori, P.; Villalobos, E.M.; Martin, S.J.; Jones, I.M.; Schroeder, D.C. Moku virus; a new iflavirus found in wasps, honey bees and varroa. Sci. Rep. 2016, 6, 34983. [CrossRef]

37. Viljakainen, L.; Holmberg, I.; Abril, S.; Jurvansuu, J. Viruses of invasive argentine ants from the european main supercolony: Characterization, interactions and evolution. J. Gen. Virol. 2018, 99, 1129-1140. [CrossRef]

38. Evans, J.D.; Schwarz, R.S.; Chen, Y.P.; Budge, G.; Cornman, R.S.; De la Rua, P.; de Miranda, J.R.; Foret, S.; Foster, L.; Gauthier, L.; et al. Standard methods for molecular research in apis mellifera. J. Apic. Res. 2013, 52, 1-54. [CrossRef] 
39. Yue, C.; Genersch, E. Rt-pcr analysis of deformed wing virus in honeybees (apis mellifera) and mites (varroa destructor). J. Gen. Virol. 2005, 86, 3419-3424. [CrossRef]

40. Craggs, J.K.; Ball, J.K.; Thomson, B.J.; Irving, W.L.; Grabowska, A.M. Development of a strand-specific rt-pcr based assay to detect the replicative form of hepatitis c virus rna. J. Virol Methods 2001, 94, 111-120. [CrossRef]

41. Guu, T.S.; Zheng, W.; Tao, Y.J. Bunyavirus: Structure and replication. In Viral Molecular Machines; Springer: Boston, MA, USA, 2012; pp. 245-266.

42. De Miranda, J.R.; Bailey, L.; Ball, B.V.; Blanchard, P.; Budge, G.E.; Chejanovsky, N.; Chen, Y.-P.; Gauthier, L.; Genersch, E.; de Graaf, D.C.; et al. Standard methods for virus research in apis mellifera. J. Apic. Res. 2015, 52, 1-56. [CrossRef]

43. R Core Team. Team. R: A language and Environment for Statistical Computing. Vienna, Austria: R Foundation for Statistical Computing. Available online: Https://www.R-project.Org (accessed on 2 September 2019).

44. Stevenson, M.; Nunes, T.; Sanchez, J.; Thornton, R.; Reiczigel, J.; Robison-Cox, J.; Sebastiani, P. Epir: An r package for the analysis of epidemiological data. R package version 1.0-4, 2013.

45. Felden, A.; Paris, C.; Chapple, D.G.; Suarez, A.V.; Tsutsui, N.D.; Lester, P.J.; Gruber, M.A. Native and introduced argentine ant populations are characterised by distinct transcriptomic signatures associated with behaviour and immunity. NeoBiota 2019, 49, 105-126. [CrossRef]

46. Thompson, J.; Higgins, D.; Gibson, T. Clustalw: Improving the sensitivity of progressive multiple sequence through weighing, position-specific gap penalties and weight matrix choice. Nucleic Acids Res. 1994, 22, 4673-4680. [CrossRef]

47. Kearse, M.; Moir, R.; Wilson, A.; Stones-Havas, S.; Cheung, M.; Sturrock, S.; Buxton, S.; Cooper, A.; Markowitz, S.; Duran, C.; et al. Geneious basic: An integrated and extendable desktop software platform for the organization and analysis of sequence data. Bioinformatics 2012, 28, 1647-1649. [CrossRef]

48. Kumar, S.; Stecher, G.; Li, M.; Knyaz, C.; Tamura, K. Mega x: Molecular evolutionary genetics analysis across computing platforms. Mol. Biol. Evol. 2018, 35, 1547-1549. [CrossRef]

49. Bouckaert, R.; Heled, J.; Kühnert, D.; Vaughan, T.; Wu, C.-H.; Xie, D.; Suchard, M.A.; Rambaut, A.; Drummond, A.J. Beast 2: A software platform for bayesian evolutionary analysis. PLoS Comput. Biol. 2014, 10, e1003537. [CrossRef]

50. Rieux, A.; Balloux, F. Inferences from tip-calibrated phylogenies: A review and a practical guide. Mol. Ecol. 2016, 25, 1911-1924. [CrossRef]

51. Drummond, A.J.; Pybus, O.G.; Rambaut, A.; Forsberg, R.; Rodrigo, A.G. Measurably evolving populations. Trends Ecol. Evol. 2003, 18, 481-488. [CrossRef]

52. Parker, J.; Rambaut, A.; Pybus, O.G. Correlating viral phenotypes with phylogeny: Accounting for phylogenetic uncertainty. Infect. Genet. Evol. 2008, 8, 239-246. [CrossRef]

53. Wang, T.H.; Donaldson, Y.K.; Brettle, R.P.; Bell, J.E.; Simmonds, P. Identification of shared populations of human immunodeficiency virus type 1 infecting microglia and tissue macrophages outside the central nervous system. J. Virol. 2001, 75, 11686-11699. [CrossRef]

54. Boncristiani, H.F., Jr.; Di Prisco, G.; Pettis, J.S.; Hamilton, M.; Chen, Y.P. Molecular approaches to the analysis of deformed wing virus replication and pathogenesis in the honey bee, apis mellifera. Virol. J. 2009, 6, 221. [CrossRef] [PubMed]

55. Bonning, B.C.; Miller, W.A. Dicistroviruses. Annu. Rev. Entomol. 2010, 55, 129-150. [CrossRef] [PubMed]

56. Meeus, I.; de Miranda, J.R.; de Graaf, D.C.; Wackers, F.; Smagghe, G. Effect of oral infection with kashmir bee virus and israeli acute paralysis virus on bumblebee (bombus terrestris) reproductive success. J. Invertebr. Pathol. 2014, 121, 64-69. [CrossRef] [PubMed]

57. Benaets, K.; Geystelen, A.V.; Cardoen, D.; Smet, L.D.; Graaf, D.C.d.; Schoofs, L.; Larmuseau, M.H.D.; Brettell, L.E.; Martin, S.J.; Wenseleers, T. Covert deformed wing virus infections have long-term deleterious effects on honeybee foraging and survival. Proc. Biol. Sci. 2017, 284, 20162149. [CrossRef]

58. Martin, S.J.; Brettell, L.E. Deformed wing virus in honeybees and other insects. Annu. Rev. Virol. 2019, 6, 49-69. [CrossRef]

59. Remnant, E.J.; Shi, M.; Buchmann, G.; Blacquiere, T.; Holmes, E.C.; Beekman, M.; Ashe, A. A diverse range of novel rna viruses in geographically distinct honey bee populations. J. Virol. 2017, 91, e00158-17. [CrossRef] 
60. Seabloom, E.W.; Borer, E.T.; Gross, K.; Kendig, A.E.; Lacroix, C.; Mitchell, C.E.; Mordecai, E.A.; Power, A.G. The community ecology of pathogens: Coinfection, coexistence and community composition. Ecol. Lett. 2015, 18, 401-415. [CrossRef]

61. Daszak, P.; Cunningham, A.A.; Hyatt, A.D. Anthropogenic environmental change and the emergence of infectious diseases in wildlife. Acta Trop. 2001, 78, 103-116. [CrossRef]

62. Lembo, T.; Hampson, K.; Haydon, D.T.; Craft, M.; Dobson, A.; Dushoff, J.; Ernest, E.; Hoare, R.; Kaare, M.; Mlengeya, T.; et al. Exploring reservoir dynamics: A case study of rabies in the serengeti ecosystem. J. Appl. Ecol. 2008, 45, 1246-1257. [CrossRef]

63. Wilfert, L.; Long, G.; Leggett, H.C.; Schmid-Hempel, P.; Butlin, R.; Martin, S.J.; Boots, M. Deformed wing virus is a recent global epidemic in honeybees driven by varroa mites. Science 2016, 351, 594-597. [CrossRef]

64. Ravoet, J.; De Smet, L.; Meeus, I.; Smagghe, G.; Wenseleers, T.; de Graaf, D.C. Widespread occurrence of honey bee pathogens in solitary bees. J. Invertebr. Pathol. 2014, 122, 55-58. [CrossRef] [PubMed]

65. Manley, R.; Temperton, B.; Boots, M.; Wilfert, L. Contrasting impacts of a novel specialist vector on multihost viral pathogen epidemiology in wild and managed bees. Mol. Ecol. 2020, 29, 380-393. [CrossRef] [PubMed]

66. Dobson, A. Population dynamics of pathogens with multiple host species. Am. Nat. 2004, 164, 64-78. [CrossRef] [PubMed]

67. Alizon, S.; de Roode, J.C.; Michalakis, Y. Multiple infections and the evolution of virulence. Ecol. Lett. 2013, 16, 556-567. [CrossRef] [PubMed]

68. Telfer, S.; Lambin, X.; Birtles, R.; Beldomenico, P.; Burthe, S.; Paterson, S.; Begon, M. Species interactions in a parasite community drive infection risk in a wildlife population. Science 2010, 330, 243-246. [CrossRef] [PubMed]

69. Natsopoulou, M.E.; McMahon, D.P.; Doublet, V.; Frey, E.; Rosenkranz, P.; Paxton, R.J. The virulent, emerging genotype $\mathrm{b}$ of deformed wing virus is closely linked to overwinter honeybee worker loss. Sci. Rep. 2017, 7, 5242. [CrossRef]

70. Mordecai, G.J.; Brettell, L.E.; Martin, S.J.; Dixon, D.; Jones, I.M.; Schroeder, D.C. Superinfection exclusion and the long-term survival of honey bees in varroa-infested colonies. ISME J. 2016, 10, 1182-1191. [CrossRef]

71. Mideo, N. Parasite adaptations to within-host competition. Trends Parasitol. 2009, 25, 261-268. [CrossRef]

72. Natsopoulou, M.E.; McMahon, D.P.; Doublet, V.; Bryden, J.; Paxton, R.J. Interspecific competition in honeybee intracellular gut parasites is asymmetric and favours the spread of an emerging infectious disease. Proc. Biol. Sci. 2015, 282, 20141896. [CrossRef]

73. Lello, J.; Boag, B.; Fenton, A.; Stevenson, I.R.; Hudson, P.J. Competition and mutualism among the gut helminths of a mammalian host. Nature 2004, 428, 840-844. [CrossRef]

74. Seppälä, O.; Karvonen, A.; Valtonen, E.T.; Jokela, J. Interactions among co-infecting parasite species: A mechanism maintaining genetic variation in parasites? Proc. Biol. Sci. 2009, 276, 691-697. [CrossRef] [PubMed]

75. Rigaud, T.; Perrot-Minnot, M.J.; Brown, M.J. Parasite and host assemblages: Embracing the reality will improve our knowledge of parasite transmission and virulence. Proc. Biol. Sci. 2010, 277, 3693-3702. [CrossRef] [PubMed]

76. Ramsey, S.D.; Ochoa, R.; Bauchan, G.; Gulbronson, C.; Mowery, J.D.; Cohen, A.; Lim, D.; Joklik, J.; Cicero, J.M.; Ellis, J.D.; et al. Varroa destructor feeds primarily on honey bee fat body tissue and not hemolymph. Proc. Natl. Acad. Sci. USA 2019, 116, 1792-1801.

77. Gisder, S.; Aumeier, P.; Genersch, E. Deformed wing virus: Replication and viral load in mites (varroa destructor). J. Gen. Virol. 2009, 90, 463-467. [CrossRef] [PubMed]

78. Posada-Florez, F.; Childers, A.K.; Heerman, M.C.; Egekwu, N.I.; Cook, S.C.; Chen, Y.; Evans, J.D.; Ryabov, E.V. Deformed wing virus type a, a major honey bee pathogen, is vectored by the mite varroa destructor in a non-propagative manner. Sci. Rep. 2019, 9, 12445. [CrossRef] [PubMed]

79. Porter, S.D.; Valles, S.M.; Pereira, R.M. Scavenging crickets (orthoptera: Gryllidae) transmit solenopsis invicta virus 3 to red imported fire ant (hymenoptera: Formicidae) colonies. Fla. Entomol. 2016, 99, 811-812. [CrossRef] 
80. Le Sage, M.J.; Towey, B.D.; Brunner, J.L. Do scavengers prevent or promote disease transmission? The effect of invertebrate scavenging on ranavirus transmission. Funct. Ecol. 2019, 33, 1342-1350. [CrossRef]

81. Mondet, F.; de Miranda, J.R.; Kretzschmar, A.; Le Conte, Y.; Mercer, A.R. On the front line: Quantitative virus dynamics in honeybee (apis mellifera 1.) colonies along a new expansion front of the parasite varroa destructor. PLoS Pathog. 2014, 10, e1004323. [CrossRef] 


\section{Appendix 2:}

Viruses and their effects in ants (Hymenoptera: Formicidae) by James W.Baty, Mariana

Bulgarella, Jana Dobelmann, Antoine Felden, and Philip J. Lester as published in Myrmecological news (30) pp 213-228 


\title{
Viruses and their effects in ants (Hymenoptera: Formicidae)
}

\author{
James W. Baty, Mariana Bulgarella, Jana Dobelmann, Antoine Felden \& Philip J. Lester
}

\begin{abstract}
Viruses are ubiquitous within all forms of cellular life, including ants. We documented the currently known viral infections described and their effects on ants. Our literature review found 87 different viruses (including 40 putative viruses and five bacteriophages detected via high-throughput sequencing) across 38 ant species. The majority of these viruses have been described from studying pathogens as potential biological control agents for the invasive red imported fire ant, Solenopsis invicta BuREN, 1972 or due to efforts to determine if ants serve as reservoirs for honey bee viruses in places where Argentine ants, Linepithema humile (MAYR, 1868) are also invasive. Most of these viruses belong to the Picornavirales order of small RNA single-stranded RNA (ssRNA) viruses, with more than half being positive-sense (+ssRNA) viruses. We review modes of viral transmission and suggest that horizontal transmission is a common mode of infection in ants as they share food via trophallaxis, although vertical transmission of viruses in eggs from queens has been observed. Viruses can substantially alter ant behaviour and physiology. We review effects of viruses on immune gene expression, feeding, locomotion, aggression, and colony defence. Then, we review the current state of the art in prospecting and using viruses for biological control. Mortality of ant colonies can occur, although the impact of some viral infections appears to be dependent on other environmental factors. Solenopsis invicta virus 3 (SINV-3) has had the most focus as a biological control agent. Effective laboratory and field transmission of SINV-3 in S. invicta colonies has been demonstrated although large-scale ant control with SINV-3 has not yet been reported. Finally, we review virus discovery and detection methods, including high-throughput sequencing that has revolutionised the field. We encourage testing for viral replication within each ant species to confirm active infection and that the ant is a true host to the virus, and we recommend approaches for viral discovery in invasive ants that focus on colony monitoring in their native range.
\end{abstract}

Key words: Viral pathogens, physiology, behaviour, population dynamics, viral discovery, review.

Received 11 June 2020; revision received 21 August 2020; accepted 7 September 2020

Subject Editor: Chris R. Smith

James W. Baty (contact author), Mariana Bulgarella, Jana Dobelmann, Antoine Felden \& Philip J. Lester, School of Biological Sciences, Victoria University of Wellington, PO Box 600, Wellington, New Zealand. E-Mail: James.Baty@vuw.ac.nz

\section{Introduction}

Viruses are ubiquitous, parasitizing all cellular life forms, and are thought to represent the most physically-abundant and genetically-diverse biological entities on Earth (Koonin \& DolJA 2013). Ants have been known to harbour viruses since the mid-20 ${ }^{\text {th }}$ century with reports of viral-like particles in the common wood ant Formica lugubris ZETTERSTEDT, 1838 (STEIGER \& al. 1969), and in the fire ant, Solenopsis spp. (AvERY \& al. 1977). Since these publications, many different viral families have been discovered in different ant species, with much of the research typically being driven by the hunt for biological control strategies for globally important invasive ant species such as the red imported fire ant (Solenopsis invicta Buren, 1972) (VAlles 2012, OI \& al. 2015). The objectives of this review are to: (1) summarise diversity, discovery, modes of transmission, and specificity of viruses infecting ants; (2) outline the effects of viruses on ant physiology and behaviour; and (3) discuss the potential of viruses for biological control of ants. Our goal with this resource is to encourage further research and highlight knowledge gaps ready to be explored.

\section{The diversity, specificity, and phylogenetic relationships of viruses infecting ants}

Our literature review of publications reporting viruses in ants showed 87 different viruses across 38 ant species (Tab. 1 and Tab. $\mathrm{S} 1$ as digital supplementary material to this article, at the journal's web pages). Many of the detected viruses have been found in the invasive Argentine ant Linepithema humile (MAYR, 1868) and red imported fire 
Tab. 1: Summary of viruses reported to occur in ants. Viruses demonstrated to replicate in the ant host are shown in bold and with an asterisk. Viral replication demonstrates true parasitism of an ant host. Some of the viruses detected might not infect ants but were present following ingestion or for other reasons. In addition, 33 of the viruses listed were identified from partial sequences obtained via high-throughput sequencing. Viral detection methods and references are found in Table S1 as digital supplementary material to this article, at the journal's web pages.

\begin{tabular}{|c|c|}
\hline Ant species & Viruses \\
\hline \multicolumn{2}{|c|}{ Subfamily - Dolichoderinae } \\
\hline Forelius sp. & Deformed wing virus (DWV) \\
\hline Linepithema humile & 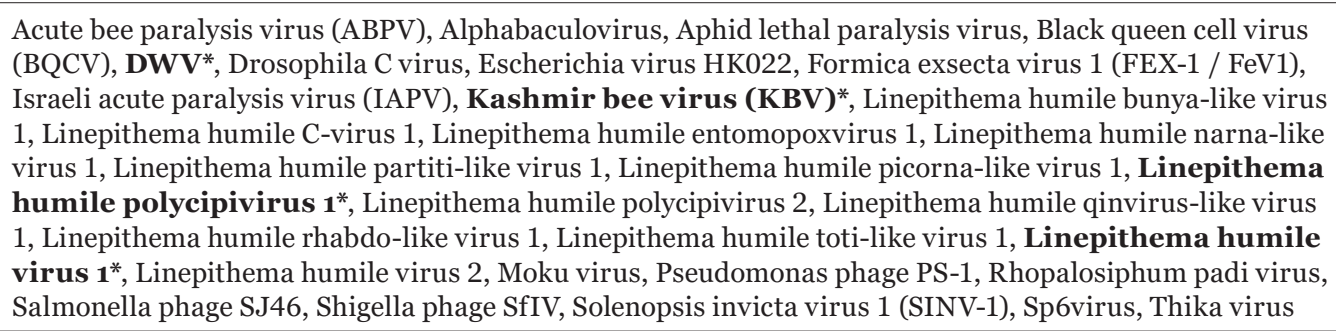 \\
\hline $\begin{array}{l}\text { Tapinoma } \\
\text { melanocephalum }\end{array}$ & $\begin{array}{l}\text { ABPV, BQCV, DWV, IAPV, KBV, Lake Sinai virus (LSV), Milolii virus, Moku virus, Sacbrood virus (SBV), } \\
\text { Slow bee paralysis virus (SBPV) }\end{array}$ \\
\hline \multicolumn{2}{|c|}{ Subfamily - Formicinae } \\
\hline $\begin{array}{l}\text { Anoplolepis } \\
\text { gracilipes }\end{array}$ & TR17983 virus, TR44839 virus*, TR80102 virus \\
\hline Brachymyrmex sp. & BQCV, DWV, SBV \\
\hline Camponotus sp. & ABPV, BQCV, DWV, IAPV, SBV \\
\hline $\begin{array}{l}\text { Camponotus } \\
\text { japonicus }\end{array}$ & Wuhan Ant Virus 1 \\
\hline $\begin{array}{l}\text { Camponotus } \\
\text { nipponicus }\end{array}$ & Camponotus nipponicus virus \\
\hline Camponotus vagus & Chronic bee paralysis virus (CBPV)* \\
\hline $\begin{array}{l}\text { Camponotus } \\
\text { yamaokai }\end{array}$ & Camponotus yamaokai virus \\
\hline Colobopsis shohki & Colobopsis shohki virus 1 \\
\hline Formica aquilonia & FEX-1 / FeV1 \\
\hline Formica cinerea & FEX-1 / FeV1, Formica exsecta virus 2 (FeV2), KBV \\
\hline Formica exsecta & FEX-1 / FeV1, FeV2, Formica exsecta virus 3, Formica exsecta virus 4 (FeV4), KBV \\
\hline Formica fusca & FEX-1 / FeV1, FeV2, FeV4, Formica fusca virus $1^{*}, \mathrm{KBV}$ \\
\hline Formica pressilabris & FEX-1 / FeV1, FeV2, KBV \\
\hline Formica rufa & CBPV \\
\hline Formica truncorum & FEX-1 / FeV1, FeV2 \\
\hline Lasius neglectus & Lasius neglectus virus 1 , Lasius neglectus virus $2^{*}$ \\
\hline Lasius niger & Lasius niger virus 1, ABPV*, DWV (strains A and B) \\
\hline Lasius platythorax & ABPV*, DWV (strains A and B) \\
\hline Nylanderia sp. & ABPV, BQCV, IAPV \\
\hline Nylanderia fulva & Nylanderia fulva virus $1^{*}$ \\
\hline \multicolumn{2}{|c|}{ Subfamily - Myrmicinae } \\
\hline $\begin{array}{l}\text { Aphaenogaster } \\
\text { texana }\end{array}$ & $\mathrm{ABPV}$ \\
\hline Crematogaster sp. & ABPV, BQCV, DWV, IAPV, KBV, SBV \\
\hline $\begin{array}{l}\text { Monomorium } \\
\text { pharaonis }\end{array}$ & Monomorium pharaonis virus 1 , Monomorium pharaonis virus 2 \\
\hline Myrmica scabrinodis & Myrmica scabrinodis virus 1, Myrmica scabrinodis virus 2* \\
\hline Pheidole sp. & ABPV, BQCV, DWV, IAPV \\
\hline
\end{tabular}




\begin{tabular}{|c|c|}
\hline Ant species & Viruses \\
\hline $\begin{array}{l}\text { Pheidole } \\
\text { megacephala }\end{array}$ & ABPV, BQCV, DWV (strains A, B and C), IAPV, KBV, LSV, Milolii virus, Moku virus, SBV, SBPV \\
\hline Pogonomyrmex sp. & $\mathrm{ABPV}$ \\
\hline $\begin{array}{l}\text { Pogonomyrmex } \\
\text { californicus }\end{array}$ & KBV \\
\hline $\begin{array}{l}\text { Solenopsis } \\
\text { carolinensis }\end{array}$ & Solenopsis invicta virus $1 \mathrm{~A}(\mathrm{SINV}-1 \mathrm{~A})$ \\
\hline Solenopsis geminata & SINV-1 \\
\hline Solenopsis geminata & SINV-1A \\
\hline $\begin{array}{l}\text { Solenopsis geminata/ } \\
\text { xyloni hybrid }\end{array}$ & SINV-1 \\
\hline Solenopsis invicta & $\begin{array}{l}\text { ABPV, Alber virus, Aphid lethal paralysis virus, Autographa californica nuclear polyhedrosis virus } \\
\text { (AcNPV) AaIT-p10, AcNPV-AaIT-ie1, AcNPV-LqhIT2-ie1, Big Sioux River virus, BQCV, DWV, Drosophila C } \\
\text { virus, Helicoverpa zea nuclear polyhedrosis virus (HzNPV), HzNPV-LqhIT2-ie1, Hubei orthoptera virus 1, } \\
\text { Hubei picorna-like virus 46, Hubei picorna-like virus 50, IAPV, KBV, Mosinovirus, Nasonia vitripennis } \\
\text { virus, Nodamura virus, Rhopalosiphum padi virus, SBV, Shuangao insect virus 8, Solenopsis invicta } \\
\text { densovirus, SINV-1*, SINV-1A, Solenopsis invicta virus 2 (SINV-2)*, Solenopsis invicta virus } 3 \\
\text { (SINV-3)*, Solenopsis invicta virus } 4 \text { (SINV-4), Solenopsis invicta virus } 5 \text { (SINV-5)*, Solenopsis } \\
\text { invicta virus } 6 \text { (SINV-6), Solenopsis invicta virus } 7 \text { (SINV-7), Solenopsis invicta virus } 8 \text { (SINV-8), Solenopsis } \\
\text { invicta virus } 9 \text { (SINV-9), Solenopsis invicta virus } 10 \text { (SINV-10), Solenopsis invicta virus } 11 \text { (SINV-11), } \\
\text { Solenopsis invicta virus 12 (SINV-12), Solenopsis invicta virus } 13 \text { (SINV-13), Solenopsis midden virus, } \\
\text { Wuhan arthropod virus 2, Wuhan insect virus } 11\end{array}$ \\
\hline Solenopsis richteri & SINV-1 \\
\hline $\begin{array}{l}\text { Solenopsis invicta/ } \\
\text { richteri hybrid }\end{array}$ & SINV-1, SINV-1A, SINV-3*, SINV-3 hybrid \\
\hline \multicolumn{2}{|c|}{ Subfamily - Pseudomyrmecinae } \\
\hline $\begin{array}{l}\text { Pseudomyrmex } \\
\text { gracilis }\end{array}$ & DWV \\
\hline
\end{tabular}

ant Solenopsis invicta due to efforts to determine if ants serve as reservoirs for honey bee viruses and to discover potential biocontrol agents, respectively. A large number of studies focused on these two ant species, therefore, it is highly likely that a substantial diversity of viruses still remains unknown in the other $>13,500$ ant species on the planet (BoROwIEC \& al. 2020). Most of the reported viruses belong to the Picornavirales order of single-stranded RNA (ssRNA) viruses. More than half of the viruses are positive-sense ssRNA (+ssRNA) viruses, however other viral genome types have also been found including negative-sense ssRNA (-ssRNA); double-stranded RNA (dsRNA); and double-stranded DNA (dsDNA). Replication of +ssRNA viruses involves production of the negative-sense RNA strand which indicates that the virus is replicating and that the ant serves as a host. We report on $13+$ ssRNA viruses known to replicate within ant hosts representing four families (Dicistroviridae, Iflaviridae, Polycipiviridae, and Solinviviridae; Fig. 1) in one viral order (Picornavirales). If we include all viral families detected in ants, not only those shown to replicate, then the viruses reported represent 15 families. We note that these numbers include 40 putative viruses that were only detected in high-throughput sequencing datasets and are sometimes represented by only partial sequences, and also include five bacteriophages (in three different bacteriophage families) that do not directly infect ants but bacteria instead.
Virus bioprospecting in ants has led to unique virus discoveries not detected previously, which now represent new taxa. Recently, two new virus families originated from discoveries in ant hosts. These two families have been accepted and ratified by the International Committee on Taxonomy of Viruses (OLENDRAITE \& al. 2017, BROwn \& al. 2019, Olendraite \& al. 2019). The family Polycipiviridae belongs to the order Picornavirales, and is characterised by a clade of arthropod-infecting polycistronic picorna-like viruses. It comprises three genera: Sopolycivirus, Hupolycivirus, and Chipolycivirus (OLENDRAITE \& al. 2017, Olendraite \& al. 2019). The genus Sopolycivirus appears to be specific for the Formicidae (OLENDRAITE \& al. 2017). Another recently discovered viral family, Solinviviridae (BROwN \& al. 2019) is a family of picorna/calici-like viruses, with two genera: Invictavirus and Nyfulvavirus. Two viral species, Solenopsis invicta virus 3 (SINV-3) and Nylanderia fulva virus 1 (NfV-1), infect ants; however, related unclassified virus sequences have been isolated from a variety of other insects and arthropods (SHI \& al. 2016, VALLES \& al. 2016). It is possible that Solinviviridae forms a sister group to Caliciviridae, though phylogenetic clustering remains inconclusive (VALLEs \& al. 2014a).

Two viruses in fire ants, Solenopsis invicta virus 7 and Solenopsis invicta virus 10 were recently discovered and may represent a unique taxonomic group, as they do not fit within the current viral taxonomic structure 

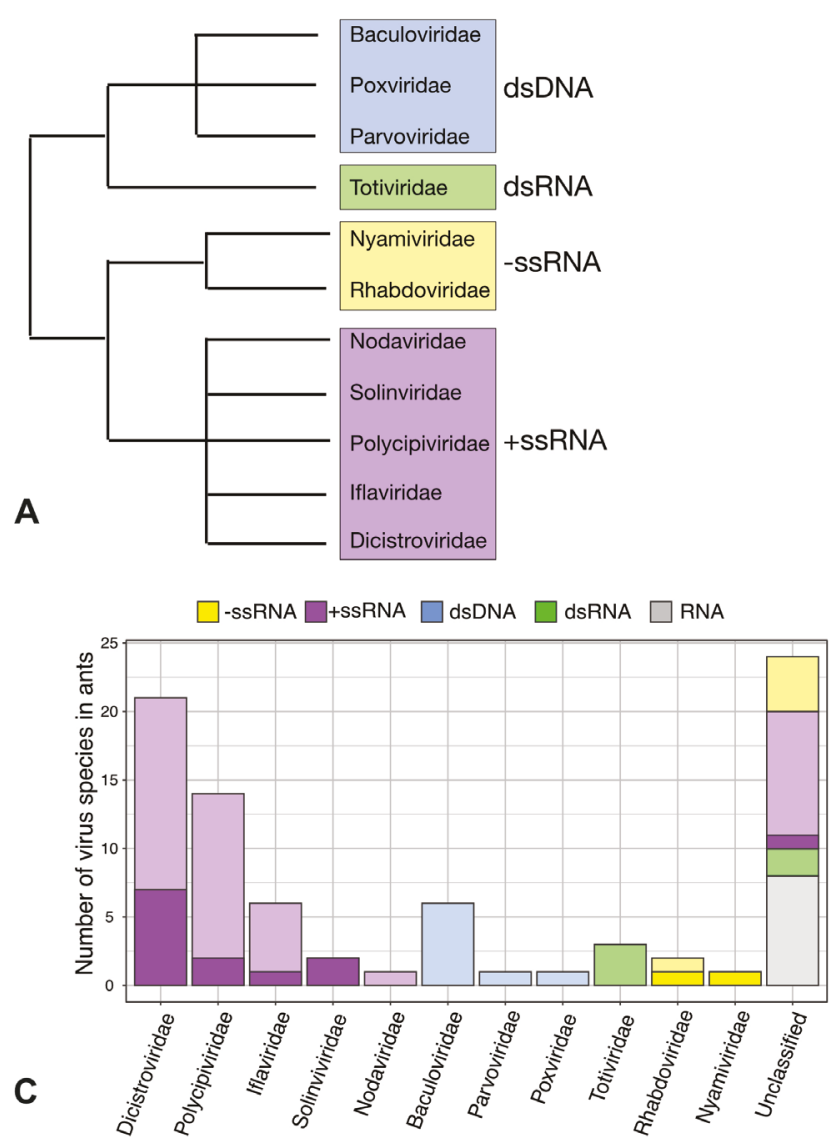
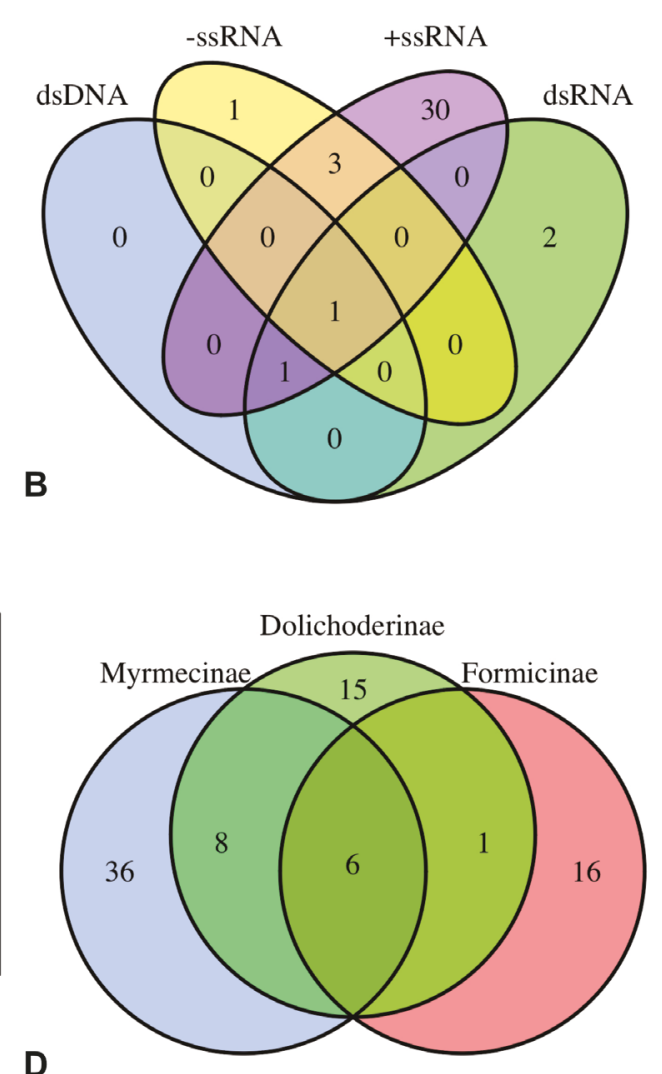

Fig. 1: (A) Tree based on Baltimore classification of viral genome organisation representing the virus families reported in ants (not including bacteriophages). (B) Venn diagram representing the number of ant species infected by different types of viruses (note the overrepresentation of +ssRNA viruses). (C) Barplot showing the number of viruses reported per virus family, including those with evidence for replication in darker colour shade. Evidence of true parasitism or that ants are definitive hosts of the viruses is demonstrated by replication. Only a portion of the viruses found in ants have been shown to replicate. The other viruses may be parasitizing the ants (but we are yet to see evidence of replication) or they may have been ingested in ant prey items. (D) Venn diagram showing the number of virus species detected in three ant subfamilies.

(VALLeS \& Rivers 2019). The same study also identified a Toti-like virus, Solenopsis midden virus, which forms a new monophyletic group divergent from the established Totiviridae genera and appears to infect arthropods exclusively (VALlES \& Rivers 2019). Another study discovered three ant viruses with sequences highly divergent from previously sequenced viruses in three different ant species, Formica fusca Linnaeus, 1758, Lasius neglectus VAN LoON, BoOMSMA \& ANDRÁSFAlvy, 1990, and Myrmica scabrinodis Nylander, 1846. Two of the viruses fall within known viral genera, however, the third, Lasius neglectus virus 2, belongs in the family Rhabdoviridae where it groups within an unclassified cluster of rhabdoviruses that infect arthropods (KLEANTHOUs \& al. 2019).

Many viruses detected in ants are also found in other species. Out of the 87 viruses reported in ants, 32 are also found in other insects and five are bacteriophages found in various organisms. Only 41 viruses are found in individual ant species and nine in multiple ant species. The five most commonly detected viruses have been described from and are more typically associated with honey bees (Apis mellifera LinnaEus, 1758): Acute bee paralysis virus (ABPV, found in 12 ant species); Deformed wing virus (DWV, found in 12 ant species); Kashmir bee virus (KBV, found in 10 ant species); Black queen cell virus (BQCV, found in nine ant species); and Israeli acute paralysis virus (IAPV, found in eight ant species). Honey bees are an economically important species, so have had much focus and examination, allowing for the discovery of these viruses. We suspect that many viruses and pathogens described from honey bees are widely shared amongst hymenopteran hosts (LoOPE \& al. 2019), and may have even evolved in non-bee hosts, but were first discovered and have had the majority of research emphasis in honey bees. For many viruses first isolated in bees, demonstration of replication in ants is still lacking (Fig. 1 and Tab.S1). We recommend testing for viral replication within each ant species in order to be confident that the virus species is parasitizing the ant and that the ant is a true host to the virus. It is also likely that some of the viruses reported in ants may have been present only after prey ingestion. Viral host specificity varies, with some viruses such as SINV-3 being completely 

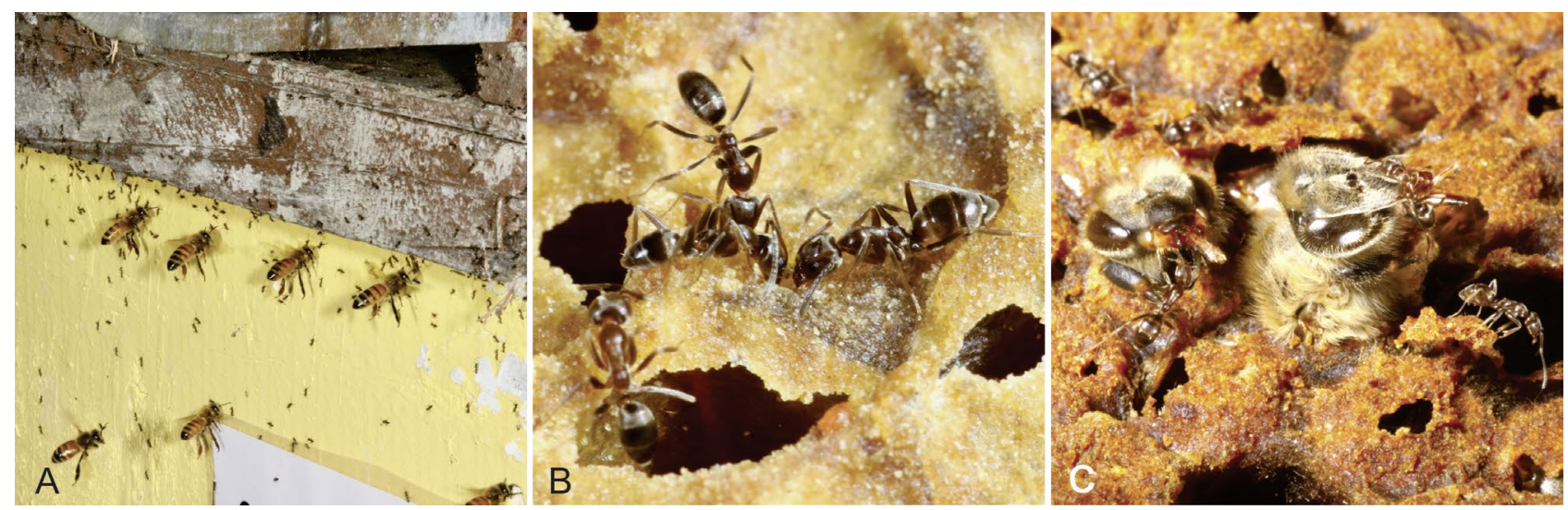

Fig. 2: Ants interact with and prey upon a wide array of insects that host viruses. Here, Argentine ants are raiding a beehive in New Zealand. (A) Honey bees appear to be stressed by ant raids but can do little to deter ants. (B) The ants consume brood and honey, and (C) swarm over emerging bee adults. Some viruses have been found to replicate in bees, Argentine ants and other arthropods (e.g., Deformed wing virus, Kashmir bee virus, Moku virus) (Dobelmann \& al. 2020). These and other viruses might be generalist viruses of insects. Other viruses found in honey bees appear to have a more restricted host range (e.g., Black queen cell virus). (Photos by Phil Lester).

specific to ants in the genus Solenopsis (PorTER \& al. 2013, PORTER \& al. 2015) whereas some other viruses are found in a diversity of arthropods.

\section{Virus transmission}

In social species there are direct and indirect opportunities for virus transmission from one individual to another. The transmission potential of a virus increases in association with three factors: abundance (host and viral load), prevalence (number of infected individuals), and infectivity (ability to initiate infection). Where societies interact with other species there is the additional risk of viral transmission to new hosts which can have substantial effects and are a major cause of emerging infectious diseases (JoHnson \& al. 2015). Of the many viral types, ssRNA viruses are especially capable of switching hosts due to the high mutation rate caused by error-prone RNA-dependent RNA polymerase (reviewed in HoLMES 2009). An example is the +ssRNA virus DWV, which has multiple hosts including various ant species, and has become a major cause of honey bee mortality around the globe (SCHROEDER \& MARTIN 2012).

Viruses can be transmitted horizontally or vertically (Chen \& al. 2006, Cremer \& al. 2007). Horizontally, viruses are transmitted between individuals in an ecosystem, whereas vertical transmission describes situations when infections spread from mother to offspring. Horizontal transmission of viruses by feeding has been confirmed for viruses in species such as the yellow crazy ant, Anoplolepis gracilipes (Sмiтн, 1857) (Hsu \& al. 2019a), Lasius spp. (ScHLÄPPI \& al. 2020), and red imported fire ants, Solenopsis spp. (VAlles \& Hashimoto 2009) as discussed in the next paragraph. Horizontal, inter-specific transmission of these viruses is likely to happen via direct or indirect interaction with infected honey bees especially for the variety of ant species found living on or near bee hives (Fig. 2) (CELLE \& al. 2008, LEviTT \& al. 2013,
Sebastien \& al. 2015, Gruber \& al. 2017, BretTell \& al. 2019, LeSTER \& al. 2019, DobelMANN \& al. 2020, PAYNE \& al. 2020, SCHLÄPPI \& al. 2020). Ants have been observed foraging on virus-infected bees discarded from bee hives and laboratory-based experiments have demonstrated that DWV and ABPV can be transmitted via ingestion by ants consuming homogenates of infected honey bee pupae (SchlÄPPI \& al. 2019, ScHLÄPPI \& al. 2020). Ants interact with bees in other ways that provide opportunities for infection, such as predation; co-habitation; and robbing of hive resources including honey (PAYNE \& al. 2020).

Within ant colonies, horizontal transmission is likely to occur through the many different interactions infected ants have with other colony members. Like other social insects, ants often live in high densities within nests and cooperate for the success of the colony, which carries risk of viral spread. Trophallaxis and allogrooming are examples of close-contact behaviour and possible routes for virus transmission. Solenopsis invicta virus 1, 2 and 3 (SINV-1, SINV-2, SINV-3) all target the midgut, and it has been hypothesized that virus particles can spread from there to the gut lumen, and then to other ants via trophallaxis or by contact with contaminated faeces (HASHIMOTO \& al. 2007, HASHIMOTO \& VALLES 2008a, VALLES \& HASHIMOTO 2009, VALLES 2012). Laboratory and field experiments with various formulations of SINV-3, including crushedants in sugar solutions, have demonstrated transmission of this virus via ingestion (VALles \& HASHimoto 2009, Porter \& al. 2013, Valles \& Porter 2013, Valles \& Oi 2014, VAlles \& Porter 2015), and showed the virus could also spread over $100 \mathrm{~m}$ from the initial bait station (OI \& al. 2019). Solenopsis invicta ants can also become infected with SINV-3 by consuming house crickets Acheta domesticus LinNAEUs, 1758 that had consumed dead infected ants (PoRTER \& al. 2016b). Solenopsis invicta virus 3 and Nylanderia fulva virus 1 are particularly infectious with unintended virus-transmission occurring in 
laboratory-reared ant colonies (VALLES \& PORTER 2013, VALLES \& al. 2016).

There is some evidence for vertical transmission for a few viruses including SINV-2, which was detected in queens and eggs of Solenopsis invicta as well as other developmental stages (Hashimoto \& VALles 2008b). Similarly, Camponotus yamaokai virus was detected in all Camponotus yamaokai Terayama \& SATOH, 1990 caste members and developmental stages including eggs (KoYAMA \& al. 2015). Currently, the prevalence of vertical transmission as a mode of transmission is unknown for most viruses in ants as there have been few studies examining transmission dynamics.

\section{Effects of viruses on ant physiology}

The majority of studies on ant viruses have focused on viral molecular characterization, phylogenetics, host specificity, transmission, geographical distribution and genomics with only a limited number of studies reporting on the effects of viruses on ant physiology. Indeed, the best-studied viruses in terms of physiological effects are three viruses infecting ants in the genus Solenopsis (VALLES \& al. 2007b): SINV-1 (VALLES \& al. 2004); SINV-2 (VALLES \& al. 2007a); and SINV-3 (VALles \& Hashimoto 2009). The effects of these viruses span from slight fitness costs to colony mortality. For the majority of ant viruses, little is known of their pathogenicity possibly because infections remain asymptomatic. We summarised the known effects (or observed absence of effects) for 13 different viruses that attack ants (Tab. 2).

Solenopsis invicta virus 1 appears to affect early stages of a queen's life cycle, from development in her natal colony up to, or shortly after, her nuptial mating flight (MANFREDINI \& al. 2016). Queens infected with SINV-1 have decreased body weight which is likely to reduce the probability of successful colony founding. It is not known whether reduced queen weight is due to the infection itself (for example SINV-1 interfering with metabolism), or whether light-weight queens are the product of colonies with chronic infection where food distribution is less efficient, or whether light-weight queens are more likely to become infected due to a lack of energy reserves (MANFREDINI \& al. 2016). Most SINV-1 infections are chronic and remain asymptomatic but may result in mortality under certain conditions of stress (VALLES 2012).

Solenopsis invicta virus 2 infections are also chronic and asymptomatic until infected individuals encounter additional stressors (НАsнiмото \& VALles 2008b). In contrast to SINV-1, SINV-2 appears to affect later stages of colony founding, with significant reductions in queen fecundity (less brood produced) and other detrimental fitness effects, including longer claustral periods, reduced weight and slower growth of newly-established colonies (MANFrEDini \& al. 2016). This virus is associated with greater changes in global gene expression in the host than SINV-1 and SINV-3 (MANFREDINI \& al. 2016). Gene expression data suggests that queens mount a stronger immune response to SINV-2 than to SINV-1 (MANFREDINI \& al. 2016).

Solenopsis invicta virus 3 infection is consistently associated with significant mortality among Solenopsis invicta laboratory colonies (Fig. 3). Once $S$. invicta colonies become infected with SINV-3, the same progression of events unfold beginning with cessation of feeding on
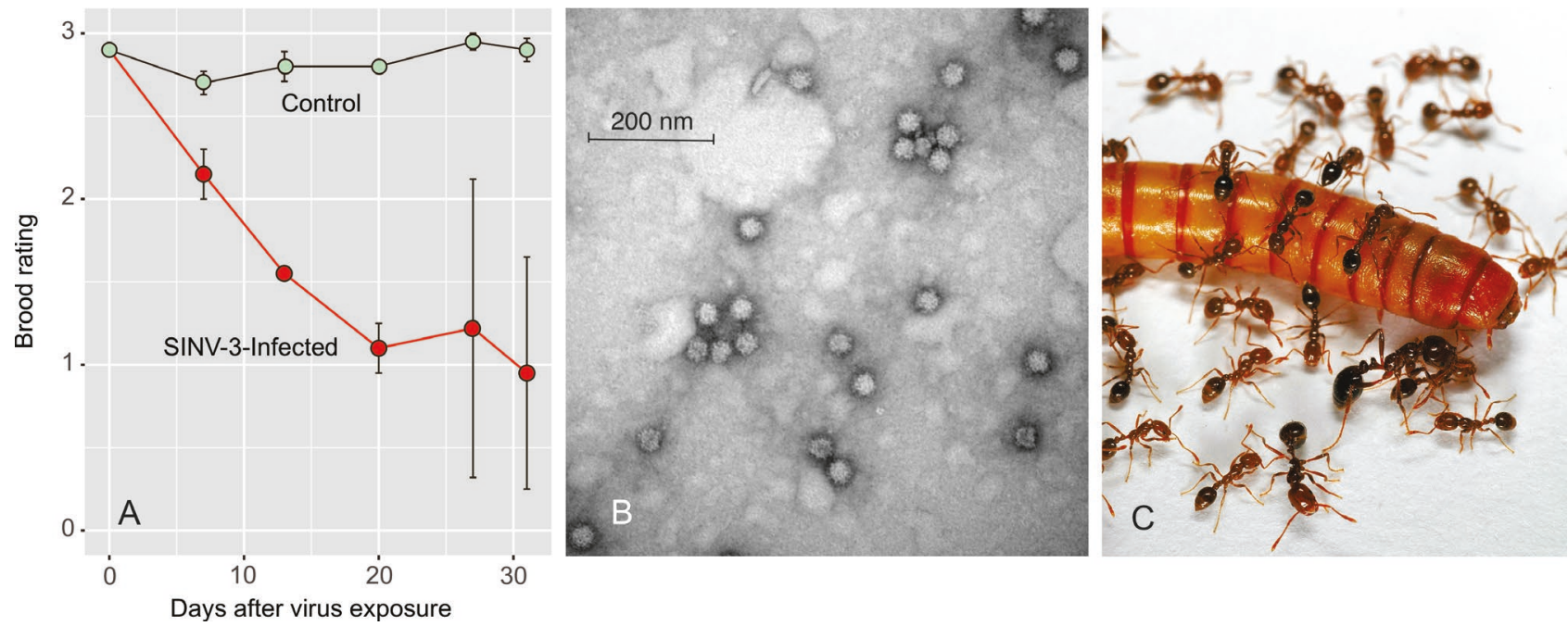

Fig. 3: (A) Solenopsis invicta virus 3 (SINV-3) is pathogenic to infected fire ant colonies. Here we show mean brood ratings ( \pm one standard error) for laboratory colonies of Solenopsis invicta infected by SINV-3 compared to uninfected control colonies, adapted from VALLES \& al. (2014b). The scoring scale ranged from colonies that were without any brood (score $=0$ ), a 2 indicating colonies were in poor health with brood mass of $\sim 50 \%$ of worker mass, to a 4 which would indicate a rapidly growing colony with brood mass $\geq$ worker mass. Figure adapted from VALLES \& al. (2014b), Solenopsis invicta virus 3: Pathogenesis and stage specificity in red imported fire ants, 67, Copyright (2014), with permission from Elsevier. (B) An electron micrograph of a negative stain of a SINV-3 preparation obtained from Solenopsis invicta worker ants and purified by cesium chloride isopycnic centrifugation (photo by Steven Valles). (C) A laboratory colony of $S$. invicta attacking a mealworm larva (Tenebrio molitor) (photo by Phil Lester). 
Tab. 2: Physiological and gene expression effects of viruses (or the reported lack of effects) in ants. Tissue tropism refers to organs in which viruses were detected.

\begin{tabular}{|c|c|c|c|}
\hline Virus & Tropism & Physiological effects on ants & $\begin{array}{l}\text { Physiological effects } \\
\text { on other insects }\end{array}$ \\
\hline $\mathrm{ABPV}$ & $\begin{array}{l}\text { In honey bees, ABPV } \\
\text { accumulates in the brain } \\
\text { and hypopharyngeal } \\
\text { glands of adults, and in } \\
\text { faeces, semen, and in the } \\
\text { brood (DE MIRANDA \& al. } \\
\text { 2010). }\end{array}$ & $\begin{array}{l}\text { Infected } L . \text { niger ants present with impaired } \\
\text { locomotion, shakiness and uncontrolled } \\
\text { movements. Infected colonies were smaller in } \\
\text { size as fewer adults emerged (SCHLÄPPI \& al. } \\
\text { 2020). }\end{array}$ & $\begin{array}{l}\text { In honey bees, the death of infected adults is } \\
\text { preceded by rapidly progressing paralysis, } \\
\text { including trembling and inability to fly. There } \\
\text { is gradual darkening and loss of hair from the } \\
\text { thorax and abdomen (BAILEY \& al. 1963, } \\
\text { MAORI \& al. 2007, RIBIËRE \& al. 2008). Severe } \\
\text { infections result in sharp declines in bee } \\
\text { populations with the appearance of diseased } \\
\text { larvae and pupae due to the lack of adults } \\
\text { available to tend brood (reviewed in DE } \\
\text { MIRANDA \& al. (2010). }\end{array}$ \\
\hline BQCV & $\begin{array}{l}\text { Detected in the gut tissue } \\
\text { of honey bees } \\
\text { (BEAUREPAIRE \& al. } \\
\text { 2020). }\end{array}$ & $\begin{array}{l}\text { In L. humile ants BQCV had the lowest rate of } \\
\text { infection or load compared with other studied } \\
\text { viruses. Viral load positively correlated with } \\
\text { KBV and was not significantly associated with } \\
\text { the expression of any immune gene studied } \\
\text { (LESTER \& al. 2019). }\end{array}$ & $\begin{array}{l}\text { In honey bees, queen larvae turn yellowish } \\
\text { with sac-appearance, later turning dark } \\
\text { brown. Infected pupae turn brown and die. } \\
\text { Queen cell walls turn dark brown/black. } \\
\text { Adults exhibit shortened lifespan (reviewed in } \\
\text { BEAUREPAIRE \& al. 2020). }\end{array}$ \\
\hline CBPV & $\begin{array}{l}\text { In honey bees, this virus } \\
\text { exhibits neurotropism, } \\
\text { and is detected in the } \\
\text { alimentary tract, } \\
\text { mandibular and } \\
\text { hypopharyngeal glands } \\
\text { (RIBIÈRE \& al. 2010). }\end{array}$ & $\begin{array}{l}\text { In a study of infected C. vagus and } F . \text { rufa, } \\
\text { there were no observations of trembling ants } \\
\text { in the field, although dead ants were found } \\
\text { along with dead bees around apiaries (CELLE } \\
\text { \& al. 2008). }\end{array}$ & $\begin{array}{l}\text { Symptoms in bees include: bloated abdomens; } \\
\text { partially-spread dislocated wings trembling } \\
\text { motion; inability to fly; and a tendency to } \\
\text { crawl on the ground and up plant stems, } \\
\text { sometimes in masses of thousands of } \\
\text { individuals. Bees often huddle together on top } \\
\text { of the cluster in the hive. Distension of the } \\
\text { honey sac with fluid leads to 'dysentery'. Sick } \\
\text { individuals die within a few days of the onset } \\
\text { of symptoms. Infected bees lose hair and } \\
\text { appear dark and almost black, shiny and } \\
\text { greasy. They suffer nibbling attacks by healthy } \\
\text { bees of their colony (appearing like robber } \\
\text { bees). Severely infected colonies suddenly } \\
\text { collapse, leaving queens with a few workers } \\
\text { (BAILEY \& BALL 1991, BALL \& BAILEY } 1997 \\
\text { and reviewed in RIBIĖRE \& al. 2010). }\end{array}$ \\
\hline DWV & $\begin{array}{l}\text { In honey bee queens, } \\
\text { DWV concentrates in the } \\
\text { reproductive tissues the } \\
\text { ovaries and spermatheca } \\
\text { but was also directly } \\
\text { observed in fat body cells } \\
\text { (FIEVET \& al. 2006). }\end{array}$ & $\begin{array}{l}\text { DWV replicates in } M . \text { rubra however, colony } \\
\text { development showed no abnormalities and } \\
\text { worker ants did not show clinical symptoms } \\
\text { (SCHLÄPPI \& al. 2019). Viral loads varied } \\
\text { enormously among infected } L \text {. humile ants, } \\
\text { and viral loads were orders of magnitude } \\
\text { higher in the presence of honey bees. Levels of } \\
\text { infection were an order of magnitude higher } \\
\text { than for KBV and BQCV (LESTER \& al. 2019). }\end{array}$ & $\begin{array}{l}\text { In honey bees, wings are crumpled or } \\
\text { aborted, and abdomens are shortened. The } \\
\text { lifespan of adult workers and drones is } \\
\text { shortened considerably. Individuals show } \\
\text { impaired learning and forging behaviour } \\
\text { (reviewed in BEAUREPAIRE \& al. 2020). }\end{array}$ \\
\hline FeV1 & & $\begin{array}{l}\text { No overt symptoms noted in } F \text {. exsecta } \\
\text { (JoHANSSON \& al. 2013). }\end{array}$ & \\
\hline FeV2 & & $\begin{array}{l}\text { Males and queen F. exsecta ants with } \\
\text { deformed wings have been observed from } \\
\text { sampled populations but the culprit has not } \\
\text { been confirmed (JoHANSSON \& al. 2013, } \\
\text { DHAYGUDE \& al. 2019). }\end{array}$ & \\
\hline FeV4 & & $\begin{array}{l}\text { No overt symptoms noted in F. exsecta } \\
\text { (JoHANsSON \& al. 2013). }\end{array}$ & \\
\hline KBV & $\begin{array}{l}\text { In honey bees, KBV is } \\
\text { detected in faeces, } \\
\text { surface-sterilised eggs } \\
\text { but not in the queen } \\
\text { ovaries (DE MIRANDA \& } \\
\text { al. 2010). }\end{array}$ & $\begin{array}{l}\text { There has been no indication of L. humile } \\
\text { colony suffering or collapse (ABRIL \& } \\
\text { JuRVANSUU 2020). In queens from the } \\
\text { European supercolony, KBV was the most } \\
\text { common virus. A high percentage of queens } \\
\text { had unusually high viral loads, indicating } \\
\text { active infection (VILJAKAINEN \& al. 2018a). }\end{array}$ & $\begin{array}{l}\text { In honeybees, infections are associated with } \\
\text { sharp decline in adult bees resulting in } \\
\text { diseased larvae and pupae due to the lack of } \\
\text { adults available to tend the brood (reviewed in } \\
\text { (DE MIRANDA \& al. 2010). }\end{array}$ \\
\hline
\end{tabular}




\begin{tabular}{|c|c|c|c|}
\hline Virus & Tropism & Physiological effects on ants & $\begin{array}{l}\text { Physiological effects } \\
\text { on other insects }\end{array}$ \\
\hline LHUV-1 & & $\begin{array}{l}\text { No indication of } L \text {. humile colony suffering or } \\
\text { collapse (ABRIL \& JURVANSUU 2020). } \\
\text { However, they mount a substantial immune } \\
\text { response to infection, involving almost all } \\
\text { immune pathways (LESTER \& al. 2019). Viral } \\
\text { loads varied enormously and were orders of } \\
\text { magnitude higher in ants outside of apiaries. } \\
\text { Viral load negatively correlated with KBV } \\
\text { load. }\end{array}$ & \\
\hline NfV-1 & $\begin{array}{l}\text { Detected in N. fulva } \\
\text { workers, larvae, pupae, } \\
\text { and queens with the } \\
\text { exception of eggs (VALLES } \\
\text { \& al. 2016). }\end{array}$ & $\begin{array}{l}\text { Infected N. fulva lack overt symptoms. One } \\
\text { infected colony in a transmission experiment } \\
\text { declined over time exhibiting significant } \\
\text { mortality among brood (83\%) and workers } \\
\text { (76\%). However, other infected colonies in the } \\
\text { experiment seemed to thrive (VALLES \& al. } \\
\text { 2016). }\end{array}$ & \\
\hline SINV-1 & \begin{tabular}{|l|} 
Replicates in the midgut \\
epithelial cells of larvae \\
and adults with infectious \\
viral particles are shed \\
into the gut lumen (OI \& \\
VALLES 2009, VALLES \\
2012).
\end{tabular} & $\begin{array}{l}\text { Infections persist chronically and } \\
\text { asymptomatically. When infected colonies } \\
\text { encounter stressors, the virus replicates } \\
\text { rapidly with host symptoms and death } \\
\text { (VALLES 2012). Significant brood mortality } \\
\text { has been observed following colony } \\
\text { translocation (VALLES \& al. 2004, Hsu \& al. } \\
\text { 2019b). SINV-1 appears to play a role in early } \\
\text { queen life, from natal colony development up } \\
\text { to/shortly after her nuptial mating flight. } \\
\text { Infected queens weighed significantly less at } \\
\text { colony foundation, likely reducing the } \\
\text { probability of successful colony founding } \\
\text { (MANFREDIN \& al. 2016). Infections } \\
\text { decreased mortality in } S \text {. invicta exposed to } \\
\text { some insecticides through unknown } \\
\text { mechanism/s, possibly through decreased } \\
\text { foraging activity at toxic baits in infected } \\
\text { colonies (TuFTS \& al. 2014, Hsu \& al. 2018). }\end{array}$ & . \\
\hline SINV-2 & $\begin{array}{l}\text { Midgut epithelial cells of } \\
\text { adults and larvae }\end{array}$ & $\begin{array}{l}\text { Infections impact later stages of colony } \\
\text { founding, resulting in queens with reduced } \\
\text { reproductive output. SINV-2 has detrimental } \\
\text { effects on fire ant incipient colonies: infected } \\
\text { colonies have longer claustral periods, weigh } \\
\text { less and grow slowly (MANFREDINI \& al. } \\
\text { 2016). }\end{array}$ & \\
\hline SINV-3 & $\begin{array}{l}\text { Detected in all tissues of } \\
\text { S. invicta queens, } \\
\text { workers and larvae } \\
\text { (VALLES \& HASHIMOTO } \\
\text { 2009, VALLES 2012). }\end{array}$ & $\begin{array}{l}\text { Severe disease symptoms following infection } \\
\text { can lead to colony death (VALLES \& } \\
\text { HASHIMOTO 2009). Infected colonies exhibit a } \\
\text { characteristic disease progression including: } \\
\text { cessation of feeding on solid food; massive } \\
\text { brood reductions; large numbers of dead ants } \\
\text { and brood; queen weight loss and decreased } \\
\text { egg production/ovary wasting; and colony } \\
\text { collapse in 30-60 days (PoRTER \& al. 2013, } \\
\text { VALLES \& al. 2014b). }\end{array}$ & \\
\hline
\end{tabular}

solid food (CHEN \& al. 2012, VALLES \& al. 2014b), followed by brood (larval and pupal) mortality (CHEN \& al. 2012, Porter \& al. 2013, VAlLes \& al. 2014b). Early signs of infection include larger than normal midden piles of adult ants followed by near complete brood disappearance (PoRTER \& al. 2013). Larval mortality is thought to occur as a result of starvation or neglect by the worker caste (VALLES \& al. 2013, VAlles \& al. 2014b, VALlES \& RIVERS 2019). Queens lose their ability for distension of the abdominal intersegmental membranes (physogastricity) (VALLES \& Hashimoto 2009). However, they continue to produce eggs. Some workers remain alive for considerable periods after the initial brood die-off, and occasionally the colonies rebound with normal brood production (VALLES \& Hashimoto 2009, VAlles 2012). In later stages of infection, worker mortality increases and queens decrease egg production and lose weight (VALLES \& al. 2013). The frequency of SINV-3 varies seasonally; its prevalence increases during cooler periods and decreases during warmer periods (VALLES \& al. 2010). Temperature can affect SINV-3 pathogenicity. Worker ants from SINV-3treated colonies maintained at low temperatures showed 
strong production of the viral capsid protein; indicating that warm summer temperatures combined with fire ant thermoregulatory behaviour might explain lower SINV-3 prevalence during summertime (VALLES \& PorTER 2019). Individual $S$. invicta ants can be infected simultaneously with all combinations of SINV-1, SINV-2 and SINV-3 (VAlles \& al. 2009, Allen \& al. 2011).

There are few studies of the physiological effects of viruses on other ant species. Recently, clinical symptoms of ABPV were found at the individual and colony levels of Lasius niger (LinNAEUs, 1758) ants (SCHLÄPPI \& al. 2020). Ants were fed bees infected with two viruses, DWV and ABPV, but only ABPV was found to replicate in these ants. Forager ants from ABPV-fed colonies presented with impaired locomotion and were shaky with uncontrolled movements. No effects of ABPV on body weight were detected, either for queens or workers. However, infected colonies were smaller due to having fewer emerging adults. The impaired movement capabilities and decreased colony size due to ABPV infections are relevant for ant fitness, and may contribute to the weakening of colonies (SCHLÄPPI $\&$ al. 2020). A larger workforce means a more productive colony, and it also provides an advantage in interspecific conflicts. Larger colonies can be expected to have a higher fitness, as they start reproducing earlier with more gynes and males produced.

Molecular work in Argentine ants examined expression patterns of immune genes in ant communities naturally infected with varying viral species and infection loads. Simultaneous viral and bacterial pathogen infections altered gene expression with both up- and down-regulation of different genes (VILJAKAINEN \& al. 2018b). Another study revealed that Argentine ants mounted greater immune responses to the bacteria Pseudomonas spp. and Linepithema humile virus 1 (LHUV-1) infections, while BQCV was not associated with strong alteration of immune gene expression (LESTER \& al. 2019). All immune pathways examined (namely the Jak / STAT, RNAi, Toll, Imd, and JNK pathways) were associated with -ssRNA viruses. The same set of genes were down-regulated in response to dsRNA and most +ssRNA viruses. Surprisingly, genes within the RNAi and Jak / STAT pathways, which are typically associated with anti-viral immune defence, were negatively associated with + ssRNA and dsRNA viruses in Argentine ants. Different microbial pathogens were clearly associated with different immune responses. Patterns of expression for nearly all the immune genes were correlated both within and between immune pathways. The expression levels of the RNAi-pathway genes Dicer and argonaute were expected to be highly correlated given their involvement in the same anti-viral response. However, nearly all other genes associated with the immune pathways studied were also significantly and highly correlated with these RNAi-pathway genes (LESTER \& al. 2019).

\section{Viral infections that change ant behaviour}

Behavioural changes associated with viral infections in ants can stem from direct pathogenic effects of the virus as well as prophylactic immune defence mechanisms (CREMER \& al. 2007). In Solenopsis invicta, viral infections have been shown to be associated with changes in foraging behaviour and food collection patterns. Colony-level foraging activity was shown to decrease after inoculation with SINV-1 (Hsu \& al. 2018). Infected colonies also displayed changes in diet preferences, with a decline of lipid intake and increased bias towards carbohydrate-rich food. Similarly, SINV-3 decreases protein intake, likely translating into poorer colony health and productivity (VALles \& al. 2013, VALLES \& al. 2014b). Solenopsis invicta virus 3-infected queens suffer from malnutrition and their ovaries are devoid of developing eggs (VALLES \& al. 2013). The changes in nutrient intake could be associated with "illness-induced anorexia", a defence mechanism believed to have evolved to deprive pathogens from essential macronutrients and allow the allocation of resources towards the immune response instead of digestion (ADAMo \& al. 2010, MAson \& al. 2014). An alternative hypothesis is that infected individuals feeding preference tilts towards carbohydrate-rich food as a compensatory feeding mechanism (SHIKANo \& CORY 2016). Solenopsis invicta virus 1 also decreases competitive abilities of $S$. invicta in staged group and individual interspecific competition assays (CHEN \& al. 2011). Interestingly, infected colonies did not engage in fights as often as uninfected colonies. A proposed mechanism was the consequence of decreased foraging activity in infected colonies, resulting in lower recruitment.

Aggression has been correlated with viral presence in another invasive ant species, the yellow crazy ant, Anoplolepis gracilipes. A virus putatively described as TR44839 was observed in invaded areas of Australia (Cooling \& al. 2016). Hsu \& al. (2019a) suggest that intercolonial aggressive behaviour in A. gracilipes was correlated with virus prevalence. They suggested that strong links between colonies with weak social boundaries (simple colony composition) resulted in epidemics of the TR44839 virus among the colonies, likely induced by horizontal transmission of viruses (Hsu \& al. 2019a). Similarly, in red imported fire ants, polygynous colonies harbor a higher diversity of parasites and pathogens when compared to their monogyne conspecifics (VALLES \& al. 2010). Resource exchanges between non-aggressive colonies are likely to facilitate viral transmission.

Researchers are only beginning to uncover the effects of viruses on ant behaviour and physiology. Virus-associated behavioural changes have been documented in a wide range of insects, including other hymenopterans (HAN \& al. 2015). In honey bees, the Kakugo variant of DWV induces increased aggression, while other DWV strains and IAPV are associated with learning disabilities and impaired foraging (FUJIYUKi \& al. 2004, 2005, IQBAL \& MUELLER 2007, LI \& al. 2013). While most changes in ant behaviour associated with viral infections are reported for foraging activity, it is likely that viruses affect a wider range of behaviours. A notable example of insect behavioural manipulation by a virus is the parasitoidism of ladybugs, Coccinella septempunctata LinNAEUs, 1758, by braconid wasps, 
Dinocampus coccinellae (SCHRANK, 1802) (DHEILly \& al. 2015). The Dinocampus coccinellae paralysis virus is injected by the ovipositing female adult wasp together with the egg, and migrates into the host's brain, presumably altering the ladybug's behaviour. Fungal, helminth and insect parasites in ants can actively manipulate host behaviour to maximise their own fitness (DE BEKKER \& al. 2018). Although viral manipulation of behaviour has not yet been observed in ants, these examples underline the possibility of virus-mediated behavioural manipulation.

Studies specifically related to viral infections are scarce, but there is a large number of studies investigating behavioural defence mechanisms against entomopathogenic fungi. A number of these reveal mechanisms that are proactive and function as prophylaxis, for example: the division of labour between individuals resulting in differences in exposure to pathogens; the use of antimicrobial compounds; or waste and corpse management (SCHMID-HEMPEL 1998, CHAPUISAT \& al. 2007, BRÜTSCH \& al. 2017, KESÄNIEMI \& al. 2019). However, ant colonies can also adjust their behaviour reactively as a result of active infections. Social interactions within colonies have been shown to undergo changes after experimental infection, suggesting that network plasticity might be a component of disease management (STROEYMEYT \& al. 2018). Remarkably, sanitary care between nestmates can also be adjusted depending on individual infection levels, likely resulting in healthier colonies (KonRAD \& al. 2018). Such behavioural immune defences have been characterised in response to non-viral pathogens and research is needed to elucidate whether virus can also elicit similar responses.

\section{Population dynamics and the potential for biological control using viruses}

Boom-and-bust dynamics have been observed in some invasive ant populations prompting the hypothesis that pathogens such as viruses may play a role in regulating ant populations (LESTER \& GRUBER 2016). Low genetic diversity, high abundance and super-colonial behaviour would seem likely to make invasive ants highly susceptible to pathogens. Viruses are known to regulate insect populations and are used in biological control programmes. Double-stranded DNA viruses of the family Baculoviridae are the most common viruses employed for biological control because they can be mass-produced, are generally accepted as safe to humans, highly pathogenic, and can be readily formulated and applied (LACEY \& al. 2015). Twenty-six different baculoviruses have been used for biological control of insect pests. In addition to baculoviruses, insect biocontrol has been attempted with two dsDNA viruses in the Parvoviridae and Nudiviridae families, respectively and with one dsRNA virus in the Reoviridae family (LACEY \& al. 2015).

Much of our current knowledge of viruses in ant populations is derived from the search for biological control agents for invasive ant species (DE BEKKER \& al. 2018), specifically with red imported fire ants and also due to efforts to determine if Argentine ants serve as reservoirs for honey bee viruses. A total of 41 viruses have been reported in red imported fire ants and 30 in Argentine ants (Tab. 1 and Tab.S1). The diversity of viruses is typically higher in the native range of both ants relative to their invaded range (VAlles 2012, Felden \& al. 2019, VAlles \& Rivers 2019). This loss of potential pathogens between natural and invaded areas is a prediction of the enemy release hypothesis (KEANE \& CRAWLEY 2002) and is a likely explanation as to why invasive ants frequently attain high densities in their invaded range (YANG \& al. 2010). The relatively lower expression of several immune pathways primarily targeting viruses in the invaded range appears to support a lower viral pressure that may allow reallocation of resources away from immunity to other functions that result in an increase of the invader's fitness (FELDEN \& al. 2019). Viral diversity appears similarly lower in the introduced range of fire ants but interestingly prevalence of SINV-1, SINV-2 and SINV-3 is higher than in the native range (YANG \& al. 2010). More data on virus prevalence between the native and introduced ranges of invasive ants is needed to further relate invasion success to release from natural enemies.

Most currently known viruses appear to typically have subtle effects on the population dynamics of invasive ants. The viruses often appear to be "covert", possibly because of the hygienic behaviour of ant colonies to remove diseased or dead individuals. Viruses such as SINV-1 and SINV-2 have been considered to conform to the paradigm of many arthropod-infecting +ssRNA viruses, in that they typically persist as chronic but asymptomatic infections that do not cause any overt signs or symptoms of disease (VALLES 2012). However, both SINV-1 and SINV-2 can lead to ant mortality when the colony is exposed to certain stressors (OI \& al. 2015). For example, infection by SINV-1 can make fire ants more susceptible to attack by other cooccurring ant species (CHEN \& al. 2011). We are yet to see any colony level effects of any of the viruses discovered in Argentine ants (SEBASTIEN \& al. 2015, GRUBER \& al. 2017, VilJAKAINEN \& al. 2018a, ABRIL \& JURVANSUU 2020) or species such as the tawny crazy ant, Nylanderia fulva (MAYR, 1862) (VALLES \& al. 2016).

Of all the viruses infecting ants, SINV-3 has received the most focus as a potential biological control agent. Multiple studies have demonstrated effective SINV-3 transmission in field colonies of Solenopsis invicta, and to our knowledge it is the only virus so far used in attempted biological control programme against an invasive ant (VAlles \& Oi 2014, Oi \& al. 2019). The combined high host specificity and high virulence have led researchers to suggest SINV-3 has the potential to be a widespread self-sustaining biocontrol agent or biopesticide (PORTER \& al. 2013, Porter \& al. 2015, Porter \& al. 2016a). The dose required to initiate a lethal response in fire ant colonies has been established. Exposure to a SINV-3 bait solution of $10^{5}$ genome equivalents per $\mu \mathrm{L}$ is sufficient to initiate and sustain an infection (VALles \& PorTer 2015). However, in order to cause lethal colony effects, a SINV-3 bait solution of at least $10^{9}$ genome equivalents per $\mu \mathrm{L}$ appears to be required. Improved efficacy might be 
reached by adding more attractive bait carriers, synergists and multiple viruses (VALles \& PorTER 2015). Solenopsis invicta virus 3 appears highly infectious and readily transmittable between colonies (VALLES \& HaSHimoto 2009, VAlles \& al. 2014b). VAlles \& al. (2014b) describe how SINV-3 infections appear to prevent or slow workers from acquiring and distributing solid food to the larvae and queen. Starvation can then occur for both the queen and larvae (VALLES \& al. 2014b). This process of colonycollapse by starvation and mortality may then take one to two months. Its effects on ant colonies, however, are both temperature- and dose-dependent as described above (VALles \& PORTER 2015, 2019). This dependency suggests that SINV-3 will not be uniformly effective everywhere, which may help explain the lack of population suppression after introduction to California for biological control (Or \& al. 2019). Furthermore, such biological control option might also be detrimental to the efficiency of baitformulated pesticide treatments if used in combination, as foraging and food intake is known to decrease with infection with viruses such as SINV-1 (TUFTs \& al. 2014, Hsu \& al. 2018).

A virus (or pathogen) effective as biological control agent in social insects is not necessarily the most virulent, as time is necessary for the virus to infect all members in a colony. Solenopsis invicta virus 3 has a much greater pathogenicity than SINV-1 or SINV-2 (OI \& al. 2019), however, its pathogenicity is lower than that of the many baculoviruses commercially-produced for biological control. For example, the one- to two-month period for fire ant colony mortality by SINV-3 (VALLES \& al. 2014b, VALLES \& PORTER 2015) contrasts with a $\mathrm{LT}_{50}$ (time to kill 50\% of a population) of 64 hours for cotton bollworm treated with the commercially produced Helicoverpa armigera single-nucleocapsid nucleopolyhedrovirus (CHEN \& al. 2000).

Highly virulent pathogens likely occur within the native range of many invasive ants. A key problem with the discovery and identification of highly lethal viruses is that a high pathogenicity will likely lead to the elimination of virulent pathogens during introduction events because of strong selection against founders (YANG \& al. 2010). This concept is supported by surveys indicating that there are many more viruses in the native range of ants than in invaded countries typically (FELDEN \& al. 2019, VALLES \& Rivers 2019). Much of the work on viral discovery in invasive ants, including ours, has involved pathogen discovery in the invaded range. We suggest that future work into viruses and other biological control agents for invasive ants involve the collection and rearing of many different colonies within the home range of the invasive ants. There are surveys that involve extensive sampling for viral and other pathogens within the native range of invasive ants (VALLEs \& al. 2018), but the sampled ants are typically killed and transported internationally for analysis. Transportation itself can be a viral and survival stressor (SAKUNA \& al. 2017) that could trigger mass viral replication causing significant mortality in ant colonies.
The focus on virus and pathogen discovery should be on colonies that experience high mortality due to highly transmittable pathogens which satisfy Koch's postulates for sequence-based pathogen identification (FREDERICKS \& RELMAN 1996). The efficiency of this work could or should be facilitated by a multi-national approach, ideally with different countries that harbour invasive ants providing resources to sustain pathogen discovery facilities in an area that encompasses the home-range of multiple invasive ants, such as Argentina. Multiple invasive ant species could be simultaneously examined and a sample bank created for future analysis.

Equally critical for biological control of invasive ants is the ability to rear and mass-produce any viral pathogen that is discovered. It has been previously recognised that a major constraint of the use of RNA viruses is a process for their large-scale production and lack of resources such as cell lines (VALles 2012, OI \& al. 2015). Methods for the development of a cell line from embryonic honey bee tissues could be adapted for ants (GoBLIRSCH \& al. 2013). An alternative option to propagating viruses in vitro is to utilize a baculovirus expression system. Allen \& VALLES (2015) utilised an insect cell line, Spodoptera frugiperda 21 that allowed production of a baculovirus expressing full-length SINV-3 transcripts. Unfortunately, despite successful transcription, in this case there was no evidence of translation (ALLEN \& VALLES 2015), although it may be a viable option for the expression of other viruses.

\section{Methodology for viral discovery and delimita- tion in ant communities}

Various approaches are employed to detect viruses in ants including: reverse transcription PCR (RT-PCR); Sanger sequencing; Next Generation Sequencing (NGS); and Rapid amplification of cDNA ends (RACE) (Tab.S1). High-throughput sequencing has revolutionised the discovery and survey of viruses. Unlike PCR-based methods, these methods do not require specific knowledge of viral genomes. Hence, recent studies have reported unprecedented numbers of new viruses using RNA high-throughput sequencing techniques (SHI \& al. 2016, GrUbER \& al. 2017, Viluakainen \& al. 2018a). Data for the study of viruses may come from messenger RNA (mRNA) sequencing (RNA-seq) or microRNA (miRNA) sequencing (sRNA-seq), as these techniques allow both for detection of RNA viruses as well as actively replicating DNA viruses. An advantage of high-throughput RNA sequencing techniques resides in the ability to characterise virus presence and loads as well as the immune response of the host. In RNA-seq studies, data allow for the analysis of immune gene expression possibly related to the host's response against viruses (LESTER \& al. 2019). In sRNA-seq experiments, the sequencing of short RNA provides information on both viral infection and also on the host RNA interference (RNAi) immune response via the production of virus-specific miRNA (Wu \& al. 2010, VILJAKAINEN \& JuRvansuU 2020). Published RNA-seq databases from studies focusing on ant gene expression may harbour 
known and even unknown viruses in reads that did not map to host genomes and are typically discarded from gene expression studies.

PCR-based methods remain extremely useful to confirm entire viral genomes and are more relevant in surveys of specific, already characterised viruses. Furthermore, PCR-based methods also allow investigation of viral replication, which is an important component of pathogen dynamics. Assays such as RT-PCR and quantitative RT-PCR are commonly used to confirm virus presence when coverage in high-throughput sequencing is low or to test for load or prevalence of specific viruses in different tissues or hosts. Quantitative RT-PCR can provide indirect evidence for active virus infections when viral genome concentrations increase in repeated sampling. Yet, in some cases viral loads may increase without active viral replication. Trophallaxis, the feeding behaviour in which fluids are shared between different members of an ant society, caused elevated SINV-3 levels in larvae of infected Solenopsis invicta colonies (VALles \& PORTER 2013), however, the lack of negative viral strands, small genome fragments (miRNA) and viral capsid protein VP2 production in larvae later revealed that SINV-3 replication is limited to the adult stage from which the larvae acquired increased SINV-3 concentrations (VALLES \& al. 2014b). Methods used to test for active viral infections include northern blot for miRNA detection, western blot to detection viral capsid proteins and strand specific RT-PCR to detect the negative strand intermediate that +SsRNA viruses produce during replication (VALLES \& al. 2014b).

To date, only 13 +ssRNA viruses have been shown to replicate in ants using strand-specific RT-PCR (Tab.S1). This common tool is used to confirm active viral infections and is usually based on a protocol first developed by Craggs \& al. (2001). During the RT step cDNA specific to the negative (antisense) strand is generated by using a virus-specific primer tagged with an unrelated sequence. To improve specificity and avoid false-positives, many studies additionally use enzymes to digest remaining RNA and single-stranded DNA (such as unincorporated RT-primers) before the PCR step. The PCR is then carried out in presence of primers specific to the virus and the tag sequence so that only cDNA derived from the tagged RT-PCR primer will be amplified (CRAGGS \& al. 2001). This method is considered the gold standard of detecting replicative forms of + SsRNA viruses and establishing host status. For instance, it was used to show that SINV-3 only replicates in the Solenopsis saevissima (SMIth, 1855) complex of fire ants and not in other ant species exposed to the virus (PorTER \& al. 2013). The host range of bee-infecting viruses has increasingly gained interest as emerging viral diseases may pose a significant risk to pollinator health. Nineteen different ant species have been found to be positive for viruses that are known to infect honey bees (Tab. 1) (CELle \& al. 2008, LeviTT \& al. 2013, GRUBER \& al. 2017, BRETTELL \& al. 2019, LESTER \& al. 2019, Dobelmann \& al. 2020, PAYNe \& al. 2020), raising concerns about virus reservoirs in ant populations
(SEbastien \& al. 2015). However, strand-specific RT-PCR has shown that only four ant species display active replication of bee-infecting viruses, including DWV and KBV replication in Linepithema humile (see GRUBER \& al. 2017), CBPV replication in Camponotus vagus (ScOPOLI, 1763) (CELLE \& al. 2008) and ABPV replication in Lasius niger and Lasius platythorax SEIFERT, 1991 (SCHLÄPPI \& al. 2020). These findings suggest that RT-PCR may be prone to detecting viruses that are not actively infecting the ant host. Viruses are equipped with a variety of strategies to protect viral RNA from degradation (DICKSON \& WILUsz 2011), perhaps causing positive RT-PCR results when ants forage on infected bees or ingest other virus-contaminated material. Research on DWV in the ectoparasitic mite Varroa destructor ANDERson \& TRUEMAN, 2000 shows that even negative strand intermediates from ingested honey bee tissue can be detected although the virus does not actively replicate within mites (PosADA-FLOREz \& al. 2019). Quantification of negative- and positive-strand copies has been used to study replication efficiency in different life stages of fire ants (HASHIMOTo \& VALLES 2008a). Quantitative strand-specific RT-PCR could help to resolve the question of active virus replication in different hosts, which can serve as an indicator of disease severity (VALles \& PORTER 2015) that can improve understanding pathogenicity of ant viruses.

\section{Concluding remarks}

Further studies are clearly warranted to understand how viral infections affect ant physiology and behaviour at the individual and colony level. It is evident that the more we look, the more we find, both in terms of viral discovery and viral impacts on their ant hosts. Rates of viral discovery in ants will increase in the following decades with new and relatively cheaper techniques. Given the recent discovery and description of a diverse community of bacteriophage viruses in the gut bacterial communities of honey bees (Bonilla-Rosso \& al. 2020, DeboutTe \& al. 2020), it seems likely that the diverse community of gut bacteria in ants will also be subject to a wide array of bacteriophages. Similarly, the recent description of how viruses can dramatically alter ant behaviour suggests that viruses may commonly affect individual hosts and colony dynamics (HsU \& al. 2019a, SCHLÄPPI \& al. 2020). Multiple pathogens also share ant hosts. It has been hypothesised that within ants, different pathogens could compete for host resources or might be synergistic in their effects on ant physiology (LESTER \& al. 2019).

The challenge remains to find viruses that are pathogenic enough to be utilized for biological control of invasive ants. The majority of viral infections that have been studied are likely those having sub-lethal or mild effects on their ant hosts. These sub-lethal viral infections are much more likely to be moved to a new geographic range and discovered in any survey, compared to viruses that quickly kill a colony. Because of their nature, the more damaging viruses are largely understudied as acutely-infected colonies most likely died before sampling. We highly 
recommend more in situ studies of viral infections on ants in their native range. In situ rearing and monitoring of large numbers of colonies to study their viruses would increase chances of finding viruses more useful for biological control.

\section{Acknowledgements}

We thank Laura Brettell for unpublished data on ant viral diversity, Steven Valles and an anonymous referee for useful comments that substantially improved our manuscript.

\section{References}

AbRIL, S. \& JURVANSUU, J. 2020: Season- and caste-specific variation in RNA viruses in the invasive Argentine ant European supercolony. - Journal of General Virology 101: 322-333.

AdAmo, S.A., BARTLETT, A., Le, J., SPENCER, N. \& Sullivan, K. 2010: Illness-induced anorexia may reduce trade-offs between digestion and immune function. - Animal Behaviour 79: 3-10.

Allen, C. \& VAlles, S.M. 2015: Successful transcription but not translation or assembly of Solenopsis invicta virus 3 in a baculovirus-driven expression system. - Florida Entomologist 98: 943-949.

Allen, C., Valles, S.M. \& Strong, C.A. 2011: Multiple virus infections occur in individual polygyne and monogyne $S o$ lenopsis invicta ants. - Journal of Invertebrate Pathology 107: 107-111.

Avery, S.W., Jouvenaz, D.P., BAnks, W.A. \& Anthony, D.W. 1977: Virus-like particles in a fire ant, Solenopsis sp., (Hymenoptera: Formicidae) from Brazil. - Florida Entomologist 60: 17-20.

BAILEY, L. \& BALL, B.V. 1991: Honey bee pathology. - Harcourt Brace Jovanovich, Sidcup, UK, 208pp.

BAIley, L., GibBs, A.J. \& Woods, R.D. 1963: Two viruses from adult honey bees (Apis mellifera LinnaEus). - Virology 21: 390-395.

BALL, B.V. \& BAILEY, L. 1997: Viruses. In: MoRSE, R.A. \& FlOTTUM, K. (Eds.): Honey bee pests, predators \& diseases. - A.I. Root Company, Medina, pp. 11-32.

Beaurepaire, A., Piot, N., Doublet, V., Antunez, K., CampBell, E., Chantawannakul, P., Chejanovsky, N., Gajda, A., Heerman, M., Panziera, D., Smagghe, G., Yañez, O., DE MiRANDA, J.R. \& DALMON, A. 2020: Diversity and global distribution of viruses of the western honey bee, Apis mellifera. - Insects 11: art. 239.

Bonilla-Rosso, G., Steiner, T., Wichmann, F., Bexkens, E. \& ENGEL, P. 2020: Honey bees harbor a diverse gut virome engaging in nested strain-level interactions with the microbiota. - Proceedings of the National Academy of Sciences of the United States of America 117: 7355-7362.

Borowiec, M.L., Moreau, C.S. \& Rabeling, C. 2020: Ants: phylogeny and classification. In: STARR, C.K. (Ed.): Encyclopedia of social insects. - Springer International Publishing, Cham, pp. 1-18.

BretTelL, L.E., Schroeder, D.C. \& MARTin, S.J. 2019: RNAseq analysis reveals virus diversity within Hawaiian apiary insect communities. - Viruses 11: art. 397.

Brown, K., Olendraite, I., VAlles, S.M., Firth, A.E., Chen, Y., GuÉrin, D.M.A., Hashimoto, Y., HerRero, S., DE MirandA, J.R. \& Ryabov, E.V. 2019: ICTV Virus taxonomy profile: Solinviviridae. - Journal of General Virology 100: 736-737.

Brütsch, T., Jaffuel, G., Vallat, A., Turlings, T.C.J. \& ChapUISAT, M. 2017: Wood ants produce a potent antimicrobial agent by applying formic acid on tree-collected resin. - Ecology and Evolution: 2249-2254.
Celle, O., Blanchard, P., Olivier, V., Schurr, F., Cougoule, N., FAUCON, J.-P. \& RibiÈRE, M. 2008: Detection of chronic bee paralysis virus (CBPV) genome and its replicative RNA form in various hosts and possible ways of spread. - Virus Research 133: 280-284.

Chapuisat, M., Oppliger, A., Magliano, P. \& Christe, P. 2007: Wood ants use resin to protect themselves against pathogens. - Proceedings of The Royal Society B-Biological Sciences 274: 2013-2017.

Chen, X., Sun, X., Hu, Z., Li, M., O’Reilly, D.R., Zuidema, D. \& VLAK, J.M. 2000: Genetic engineering of Helicoverpa armigera single-nucleocapsid nucleopolyhedrovirus as an improved pesticide. - Journal of Invertebrate Pathology 76: $140-146$.

Chen, Y., Becnel, J.J. \& VAlles, S.M. 2012: RNA viruses infecting pest insects. In: VEGA, F. \& KAYA, H.K. (Eds.): Insect pathology. $2^{\text {nd }}$ edition. - Elsevier, Amsterdam, 133-170pp.

Chen, Y., Evans, J. \& Feldlaufer, M. 2006: Horizontal and vertical transmission of viruses in the honey bee, Apis mellifera. - Journal of Invertebrate Pathology 92: 152-159.

Chen, Y.C., KAFLE, L. \& SHIH, C.J. 2011: Interspecific competition between Solenopsis invicta and two native ant species, Pheidole fervens and Monomorium chinense. - Journal of Economic Entomology 104: 614-621.

Cooling, M., Gruber, M.A.M., Hoffmann, B.D., SÉbastien, A. \& LESTER, P.J. 2016: A metatranscriptomic survey of the invasive yellow crazy ant, Anoplolepis gracilipes, identifies several potential viral and bacterial pathogens and mutualists. - Insectes Sociaux 64: 197-207.

Craggs, J.K., Ball, J.K., Thomson, B.J., Irving, W.L. \& GrabowsKa, A.M. 2001: Development of a strand-specific RT-PCR based assay to detect the replicative form of hepatitis C virus RNA. - Journal of Virological Methods 94: 111-120.

Cremer, S., ARmitage, S.A. \& SCHMid-HemPel, P. 2007: Social immunity. - Current Biology 17: R693-R702.

DE Bekker, C., Will, I., Das, B. \& Adams, R.M.M. 2018: The ants (Hymenoptera: Formicidae) and their parasites: effects of parasitic manipulations and host responses on ant behavioral ecology. - Myrmecological News 28: 1-24.

DE Miranda, J.R., Cordoni, G. \& Budge, G. 2010: The acute bee paralysis virus-Kashmir bee virus-Israeli acute paralysis virus complex. - Journal of Invertebrate Pathology 103: S30-S47.

Deboutte, W., Beller, L., Yinda, C.K., Maes, P., DE GraAf, D.C. \& MatthiJnssens, J. 2020: Honey-bee-associated prokaryotic viral communities reveal wide viral diversity and a profound metabolic coding potential. - Proceedings of the National Academy of Sciences of the United States of America 117: 10511-10519.

Dhaygude, K., Johansson, H., Kulmuni, J. \& Sundstrom, L. 2019: Genome organization and molecular characterization of the three Formica exsecta viruses-FeV1, FeV2 and FeV4. - PeerJ 6: art. e6216.

Dheilly, N.M., Maure, F., Ravallec, M., Galinier, R., Doyon, J., Duval, D., Leger, L., Volkoff, A.N., Missé, D., Nidelet, S., Demolombe, V., Brodeur, J., Gourbal, B., Thomas, F. \& MitTA, G. 2015: Who is the puppet master? Replication of a parasitic wasp-associated virus correlates with host behaviour manipulation. - Proceedings of the Royal Society B-Biological Sciences 282: art. 20142773.

Dickson, A.M. \& WiLusz, J. 2011: Strategies for viral RNA stability: live long and prosper. - Trends in Genetics 27: 286-293.

Dobelmann, J., Felden, A. \& Lester, P.J. 2020: Genetic strain diversity of multi-host RNA viruses that infect a wide range of pollinators and associates is shaped by geographic origins. - Viruses 12: art. 358. 
Felden, A., Paris, C., Chapple, D.G., Suarez, A.V., Tsutsui, N.D., Lester, P.J. \& Gruber, M.A.M. 2019: Native and introduced Argentine ant populations are characterised by distinct transcriptomic signatures associated with behaviour and immunity. - NeoBiota 49: 105-126.

Fievet, J., Tentcheva, D., Gauthier, L., DE Miranda, J.R., Cousserans, F., Colin, M.E. \& Bergoin, M. 2006: Localization of deformed wing virus infection in queen and drone Apis mellifera L. - Virology Journal 3: art. 16.

Fredericks, D.N. \& Relman, D.A. 1996: Sequence-based identification of microbial pathogens: a reconsideration of Koch's postulates. - Clinical Microbiology Reviews 9: 18-33.

Fujiyuki, T., Takeuchi, H., Ono, M., Ohka, S., Sasaki, T., Nомото, A. \& Kuво, T. 2004: Novel insect picorna-like virus identified in the brains of aggressive worker honeybees. Journal of Virology 78: 1093-1100.

Fujiyuki, T., Takeuchi, H., Ono, M., OhKa, S., Sasaki, T., Nомото, A. \& Kubо, T. 2005: Kakugo virus from brains of aggressive worker honeybees. - Advances in Virus Research 65: 1-27.

Goblirsch, M.J., SPIVAK, M.S. \& KuRTTI, T.J. 2013: A cell line resource derived from honey bee (Apis mellifera) embryonic tissues. - Public Library of Science One 8: art. e69831.

Gruber, M.A.M., Cooling, M., BATy, J.W., Buckley, K., FriedLANDER, A., Quinn, O., Russell, J., SEbASTIEn, A. \& LeSTER, P.J. 2017: Single-stranded RNA viruses infecting the invasive Argentine ant, Linepithema humile. - Scientific Reports 7: art. 3304.

Han, Y., van Oers, M.M., van Houte, S. \& Ros, V.I.D. 2015: Virus-induced behavioural changes in insects. In: MEHLHORN, H. (Ed.): Host manipulations by parasites and viruses. Springer International Publishing, Cham, pp. 149-174.

HASHimoto, Y. \& VALLES, S.M. 2008a: Detection and quantitation of Solenopsis invicta virus-2 genomic and intermediary replicating viral RNA in fire ant workers and larvae. - Journal of Invertebrate Pathology 98: 243-245.

HASHimoto, Y. \& VALLES, S.M. 2008b: Infection characteristics of Solenopsis invicta virus 2 in the red imported fire ant, Solenopsis invicta. - Journal of Invertebrate Pathology 99: $136-140$.

Hashimoto, Y., Valles, S.M. \& Strong, C.A. 2007: Detection and quantitation of Solenopsis invicta virus in fire ants by real-time PCR. - Journal of Virological Methods 140: 132-139.

Holmes, E.C. 2009: The evolutionary genetics of emerging viruses. - Annual Review of Ecology, Evolution, and Systematics 40: 353-372.

Hsu, H.W., Chiu, M.C., LeE, C.C., LeE, C.Y. \& YANG, C.S. 2019a: The association between virus prevalence and intercolonial aggression levels in the yellow crazy ant, Anoplolepis gracilipes (JERDON). - Insects 10: art. 436.

Hsu, H.W., Chiu, M.C., Shin, C.J., Matsuura, K. \& YANG, C.S. 2019b: Apoptosis as a primary defense mechanism in response to viral infection in invasive fire ant Solenopsis invicta. - Virology 531: 255-259.

Hsu, H.W., ChiU, M.C., Shoemaker, D. \& YANG, C.S. 2018: Viral infections in fire ants lead to reduced foraging activity and dietary changes. - Scientific Reports 8: art. 13498.

IQBAL, J. \& MUELLER, U. 2007: Virus infection causes specific learning deficits in honeybee foragers. - Proceedings of the Royal Society B-Biological Sciences 274: 1517-1521.

Johansson, H., DHAYgude, K., LindSTROM, S., Helanterä, H., Sundstrom, L. \& TrontTI, K. 2013: A metatranscriptomic approach to the identification of microbiota associated with the ant Formica exsecta. - Public Library of Science One 8: art. e79777.
Johnson, P.T.J., DE Roode, J.C. \& FenTon, A. 2015: Why infectious disease research needs community ecology. - Science 349: art. 1259504.

KEANE, R. \& CRAWLEY, M.J. 2002: Exotic plant invasions and the enemy release hypothesis. - Trends in Ecology \& Evolution 17: 164-170.

KesÄNiemi, J., Koskimäki, J.J. \& JuRvansuU, J. 2019: Corpse management of the invasive Argentine ant inhibits growth of pathogenic fungi. - Scientific Reports 9 art. 7593.

Kleanthous, E., Olendraite, I., Lukhovitskaya, N.I. \& FIRTH, A.E. 2019: Discovery of three RNA viruses using ant transcriptomic datasets. - Archives of Virology 164: 643-647.

Konrad, M., Pull, C.D., Metzler, S., Seif, K., NAderlinger, E., GrASSE, A.V. \& CREMER, S. 2018: Ants avoid superinfections by performing risk-adjusted sanitary care. - Proceedings of the National Academy of Sciences of the United States of America 115: 2782-2787.

Koonin, E.V. \& DoLJA, V.V. 2013: A virocentric perspective on the evolution of life. - Current Opinion in Virology 3: 546-557.

Koyama, S., Urayama, S., Ohmatsu, T., Sassa, Y., Sakai, C., Takata, M., Hayashi, S., Nagai, M., Furuya, T., Moriyama, H., SATOH, T., ONo, S. \& Mizutani, T. 2015: Identification, characterization and full-length sequence analysis of a novel dsRNA virus isolated from the arboreal ant Camponotus yamaokai. - Journal of General Virology 96: 1930-1937.

Lacey, L.A., Grzywacz, D., Shapiro-Ilan, D.I., Frutos, R., BROWNBRIDGE, M. \& GoETTEL, M.S. 2015: Insect pathogens as biological control agents: back to the future. - Journal of Invertebrate Pathology 132: 1-41.

Lester, P.J., Buick, K.H., BATY, J.W., Felden, A. \& Haywood, J. 2019: Different bacterial and viral pathogens trigger distinct immune responses in a globally invasive ant. - Scientific Reports 9: art. 5780.

LESTER, P.J. \& GRUBER, M.A.M. 2016: Booms, busts and population collapses in invasive ants. - Biological Invasions 18: 3091-3101.

LevitT, A.L., Singh, R., Cox-Foster, D.L., Rajotte, E., Hoover, K., Ostiguy, N. \& Holmes, E.C. 2013: Cross-species transmission of honey bee viruses in associated arthropods. - Virus Research 176: 232-240.

Li, Z., Chen, Y., Zhang, S., Chen, S., LI, W., YAN, L., ShI, L., WU, L., SoHr, A. \& SU, S. 2013: Viral infection affects sucrose responsiveness and homing ability of forager honey bees, Apis mellifera L. - Public Library of Science One 8 art. e77354.

LOOPE, K.J., BATY, J.W., LESTER, P.J. \& WiLsON RANKIn, E.E. 2019: Pathogen shifts in a honeybee predator following the arrival of the Varroa mite. - Proceedings of the Royal Society B-Biological Sciences 286: art. 20182499.

Manfredini, F., Shoemaker, D. \& Grozinger, C.M. 2016: Dynamic changes in host-virus interactions associated with colony founding and social environment in fire ant queens (Solenopsis invicta). - Ecology and Evolution 6: 233-244.

Maori, E., Lavi, S., Mozes-Koch, R., Gantman, Y., Peretz, Y., Edelbaum, O., Tanne, E. \& Sela, I. 2007: Isolation and characterization of Israeli acute paralysis virus, a dicistrovirus affecting honeybees in Israel: evidence for diversity due to intra- and inter-species recombination. - Journal of General Virology 88: 3428-3438.

Mason, A.P., Smilanich, A.M. \& Singer, M.S. 2014: Reduced consumption of protein-rich foods follows immune challenge in a polyphagous caterpillar. - The Journal of Experimental Biology 217: 2250-2260.

OI, D. \& VALLES, S. 2009: Fire ant control with entomopathogens in the USA. In: HAJEK, A.E., GlaRe, T.R. \& O'CALlaGHAN, M. (Eds.): Use of microbes for control and eradication of invasive arthropods. - Springer Science, New York, NY, pp. 237-258. 
Oi, D., Valles, S., Porter, S., Cavanaugh, C., White, G. \& HENKE, J. 2019: Introduction of fire ant biological control agents into the Coachella Valley of California. - Florida Entomologist 102: 284-286.

Oi, D., Porter, S.D. \& Valles, S.M. 2015: A review of the biological control of fire ants (Hymenoptera: Formicidae). - Myrmecological News 21: 101-116.

Olendraite, I., Brown, K., VAlles, S.M., Firth, A.E., Chen, Y., Guérin, D.M.A., Hashimoto, Y., Herrero, S., DE Miranda, J.R. \& Ryabov, E.V. 2019: ICTV virus taxonomy profile: Polycipiviridae. - Journal of General Virology 100: 554-555.

Olendraite, I., Lukhovitskaya, N.I., Porter, S.D., Valles, S.M. \& FirTH, A.E. 2017: Polycipiviridae: a proposed new family of polycistronic picorna-like RNA viruses. - Journal of General Virology 98: 2368-2378.

PAYNe, A.N., ShePherd, T.F. \& RANGEL, J. 2020: The detection of honey bee (Apis mellifera)-associated viruses in ants. Scientific Reports 10: art. 2923.

Porter, S.D., Gavilanez-Slone, J.M. \& VAlles, S.M. 2016a: Solenopsis invicta virus 3: infection tests with adult honey bees (Hymenoptera: Apidae). - Florida Entomologist 99: 729-733.

Porter, S.D., VAlles, S.M. \& OI, D.H. 2013: Host specificity and colony impacts of the fire ant pathogen, Solenopsis invicta virus 3. - Journal of Invertebrate Pathology 114: 1-6.

Porter, S.D., VAlles, S.M. \& Pereira, R.M. 2016b: Scavenging crickets (Orthoptera: Gryllidae) transmit Solenopsis invicta virus 3 to red imported fire ant (Hymenoptera: Formicidae) colonies. - Florida Entomologist 99: 811-812.

Porter, S.D., Valles, S.M., Wild, A.L., Dieckmann, R. \& PLOWES, N.J.R. 2015: Solenopsis invicta virus 3: further host-specificity tests with native Solenopsis ants (Hymenoptera: Formicidae). - Florida Entomologist 98: 122-125.

Posada-Florez, F., Childers, A.K., HeErman, M.C., EgeKwU, N.I., Cook, S.C., Chen, Y., Evans, J.D. \& Ryabov, E.V. 2019: Deformed wing virus type A, a major honey bee pathogen, is vectored by the mite Varroa destructor in a non-propagative manner. - Scientific Reports 9: art. 12445.

Ribière, M., BALL, B.V. \& Aubert, M.F.A. 2008: Natural history and geographic distribution of honey bee viruses. In: Aubert, M.F.A., BAll, B.V., Fries, I., Milani, N. \& Moritz, R.F.A. (Eds.): Virology and the honey bee. - EC Publications, Brussels, pp. 15-84.

Ribière, M., Olivier, V. \& Blanchard, P. 2010: Chronic bee paralysis: a disease and a virus like no other? - Journal of Invertebrate Pathology 103 Supplement 1: S120-S131.

Sakuna, K., Elliman, J. \& Owens, L. 2017: Discovery of a novel Picornavirales, chequa iflavirus, from stressed redclaw crayfish (Cherax quadricarinatus) from farms in northern Queensland, Australia. - Virus Research 238: 148-155.

Schläppi, D., Chejanovsky, N., Yanez, O. \& Neumann, P. 2020: Foodborne transmission and clinical symptoms of honey bee viruses in ants Lasius spp. - Viruses 12 art. 321.

Schläppi, D., Lattrell, P., Yanez, O., Chejanovsky, N. \& NeumanN, P. 2019: Foodborne transmission of deformed wing virus to ants (Myrmica rubra). - Insects 10 art. 394.

SCHMID-HemPel, P. 1998: Parasites in social insects. - Princeton University Press, Princeton, NJ, 392pp.

Schroeder, D.C. \& MARTin, S.J. 2012: Deformed wing virus: the main suspect in unexplained honeybee deaths worldwide. - Virulence 3: 589-591.

Sebastien, A., Lester, P.J., Hall, R.J., WANG, J., Moore, N.E. \& GRUBER, M.A. 2015: Invasive ants carry novel viruses in their new range and form reservoirs for a honeybee pathogen. - Biology Letters 11: art. 20150610.
Shi, M., Lin, X.D., Tian, J.H., Chen, L.J., Chen, X., Li, C.X., QIN, X.C., LI, J., CAO, J.P., EDEN, J.S., BUCHMANN, J., WANG, W., Xu, J., Holmes, E.C. \& Zhang, Y.Z. 2016: Redefining the invertebrate RNA virosphere. - Nature 540: 539-543.

SHIKANO, I. \& CORY, J.S. 2016: Altered nutrient intake by baculovirus-challenged insects: Self-medication or compensatory feeding? - Journal of Invertebrate Pathology 139: 25-33.

Steiger, U., Lamparter, H.E., SANdri, C. \& Akert, K. 1969: Virus-like particles in cytoplasm of neurons and glial cells of wood ants. - Archives of Virology 26: 271-282. (in German)

Stroeymeyt, N., Grasse, A.V., Crespi, A., Mersch, D.P., Cremer, S. \& Keller, L. 2018: Social network plasticity decreases disease transmission in a eusocial insect. - Science 362: 941-945.

Tufts, D.M., Hunter, W.B. \& Bextine, B. 2014: Solenopsis invicta virus (Sinv-1) infection and insecticide interactions in the red imported fire ant (Hymenoptera: Formicidae). Florida Entomologist 97: 1251-1254.

VALLES, S.M. 2012: Positive-strand RNA viruses infecting the red imported fire ant, Solenopsis invicta. - Psyche 2012: art. 821591.

VALLES, S.M., BELL, S. \& FirTH, A.E. 2014a: Solenopsis invicta virus 3: Mapping of structural proteins, ribosomal frameshifting, and similarities to Acyrthosiphon pisum virus and kelp fly virus. - Public Library of Science One 9: art. e93497.

VALLES, S.M. \& HASHiмото, Y. 2009: Isolation and characterization of Solenopsis invicta virus 3, a new positive-strand RNA virus infecting the red imported fire ant, Solenopsis invicta. - Virology 388: 354-361.

VAlLes, S.M. \& OI, D.H. 2014: Successful transmission of Solenopsis invicta virus 3 to field colonies of Solenopsis invicta (Hymenoptera: Formicidae). - Florida Entomologist 97: 1244-1246.

VALLES, S.M., OI, D.H., BECNEL, J.J., WETTERER, J.K., LAPOLLA, J.S. \& FIRTH, A.E. 2016: Isolation and characterization of Nylanderia fulva virus 1 , a positive-sense, single-stranded RNA virus infecting the tawny crazy ant, Nylanderia fulva. - Virology 496: 244-254.

VAlLES, S.M., OI, D.H. \& PoRTER, S.D. 2010: Seasonal variation and the co-occurrence of four pathogens and a group of parasites among monogyne and polygyne fire ant colonies. - Biological Control 54: 342-348.

VAlLES, S.M. \& PoRTER, S.D. 2013: Procedures to mitigate the impact of Solenopsis invicta virus 3 in fire ant (Hymenoptera: Formicidae) rearing facilities. - Florida Entomologist 96: 252-254.

VALLES, S.M. \& PORTER, S.D. 2015: Dose response of red imported fire ant colonies to Solenopsis invicta virus 3. - Archives of Virology 160: 2407-2413.

VALLES, S.M. \& PoRTER, S.D. 2019: Influence of temperature on the pathogenicity of Solenopsis invicta virus 3. - Journal of Invertebrate Pathology 166: 107217.

VAlles, S.M., Porter, S.D. \& CAlCaterRa, L.A. 2018: Prospecting for viral natural enemies of the fire ant Solenopsis invicta in Argentina. - Public Library of Science One 13: art. e0192377.

Valles, S.M., Porter, S.D., ChOI, M.-Y. \& OI, D.H. 2013: Successful transmission of Solenopsis invicta virus 3 to Solenopsis invicta fire ant colonies in oil, sugar, and cricket bait formulations. - Journal of Invertebrate Pathology 113: 198-204.

VAlles, S.M., Porter, S.D. \& FirTh, A.E. 2014b: Solenopsis invicta virus 3: pathogenesis and stage specificity in red imported fire ants. - Virology 460-461: 66-71. 
VALLES, S.M. \& Rivers, A.R. 2019: Nine new RNA viruses associated with the fire ant Solenopsis invicta from its native range. - Virus Genes 55: 368-380.

VAlles, S.M., Strong, C.A., DANG, P.M., Hunter, W.B., PereirA, R.M., Oi, D.H., Shapiro, A.M. \& Williams, D.F. 2004: A picorna-like virus from the red imported fire ant, Solenopsis invicta: initial discovery, genome sequence, and characterization. - Virology 328: 151-157.

VAlles, S.M., Strong, C.A. \& Hashimoto, Y. 2007a: A new positive-strand RNA virus with unique genome characteristics from the red imported fire ant, Solenopsis invicta. Virology 365: 457-463.

Valles, S.M., Strong, C.A., OI, D.H., Porter, S.D., Pereira, R.M., VANDER MEER, R.K., HASHIMOTO, Y., HOOPER-BUI, L.M., SANCHEZ-ARroYo, H., DAVIS, T., KARPaKAKUNJARAM, V., VAIL, K.M., Fudd Graham, L.C., BRIANo, J.A., CALCATERra, L.A., Gilbert, L.E., WARD, R., WARD, K., Oliver, J.B., TANigUChI, G. \& ThOMPson, D.C. 2007b: Phenology, distribution, and host specificity of Solenopsis invicta virus-1. - Journal of Invertebrate Pathology 96: 18-27.

Valles, S.M., Varone, L., Ramírez, L. \& Briano, J. 2009: Multiplex detection of Solenopsis invicta viruses -1, -2, and -3. - Journal of Virological Methods 162: 276-279.
VILJAKAINEN, L., Holmberg, I., ABRIL, S. \& JURVANSUU, J. 2018a: Viruses of invasive Argentine ants from the European main supercolony: characterization, interactions and evolution. Journal of General Virology 99: 1129-1140.

VilJAKAINEN, L. \& JURVANSUU, J. 2020: Discovery and analysis of RNA viruses in insects. In: SANDRELLI, F. \& TETTAMANTI, G. (Eds.): Immunity in insects. - Springer US, New York, NY, pp. 191-200.

Viljakainen, L., JurvansuU, J., Holmberg, I., Pamminger, T., ERLER, S. \& CREMER, S. 2018b: Social environment affects the transcriptomic response to bacteria in ant queens. - Ecology and Evolution 8: 11031-11070.

Wu, Q., LuO, Y., Lu, R., LAU, N., LAI, E.C., LI, W.X. \& DinG, S.W. 2010: Virus discovery by deep sequencing and assembly of virus-derived small silencing RNAs. - Proceedings of the National Academy of Sciences of the United States of America 107: 1606-1611.

Yang, C.-C., Yu, Y.-C., Valles, S.M., OI, D.H., Chen, Y.-C., Shoemaker, D., WU, W.-J. \& SHIH, C.-J. 2010: Loss of microbial (pathogen) infections associated with recent invasions of the red imported fire ant Solenopsis invicta. - Biological Invasions 12: 3307-3318. 